DOI 10.4171/JEMS/397

Jérôme Le Rousseau · Matthieu Léautaud · Luc Robbiano

\title{
Controllability of a parabolic system with a diffusive interface
}

Received June 8, 2011 and in revised form November 9, 2011

\begin{abstract}
We consider a linear parabolic transmission problem across an interface of codimension one in a bounded domain or on a Riemannian manifold, where the transmission conditions involve an additional parabolic operator on the interface. This system is an idealization of a three-layer model in which the central layer has a small thickness $\delta$. We prove a Carleman estimate in the neighborhood of the interface for an associated elliptic operator by means of partial estimates in several microlocal regions. In turn, from the Carleman estimate, we obtain a spectral inequality that yields the null controllability of the parabolic system. These results are uniform with respect to the small parameter $\delta$.
\end{abstract}

Keywords. Elliptic operator, parabolic system, transmission problem, controllability, spectral inequality

\section{Contents}

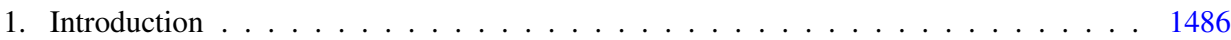

1.1. Setting . . . . . . . . . . . . . . . . . . . . 1487

1.2. Statement of the main results . . . . . . . . . . . . . . . . . . . . . . . . . . . . . . . . . . 1489

1.3. Some additional results and remarks . . . . . . . . . . . . . . . . . . . . . 1492

1.4. Notation: semiclassical operators and geometrical setting . . . . . . . . . . . . . . . . . . . 1494

2. Well-posedness and asymptotic behavior . . . . . . . . . . . . . . . . . . . . . . . . . . . . . . . . . . . . . . . .

2.1. Well-posedness . . . . . . . . . . . . . . . . . . . . . . . . . 1498

2.2. Asymptotic behavior of solutions as $\delta \rightarrow 0 \ldots \ldots \ldots \ldots \ldots$. . . . . . 1500

3. Local setting in a neighborhood of the interface . . . . . . . . . . . . . . . 1504

J. Le Rousseau: Université d'Orléans, Laboratoire de Mathématiques - Analyse, Probabilités, Modélisation - Orléans, CNRS UMR 6628, Fédération Denis-Poisson, FR CNRS 2964, B.P. 6759, 45067 Orléans Cedex 2, France; e-mail: jlr@univ-orleans.fr

M. Léautaud: Université Pierre et Marie Curie Paris 6, UMR 7598, Laboratoire Jacques-Louis Lions, Paris, F-75005 France; CNRS, UMR 7598 LJLL, Paris, F-75005 France, and Laboratoire POEMS, INRIA Paris-Rocquencourt/ENSTA, CNRS UMR 2706, France; current address: IMJ-PRG, UMR CNRS 7586, Université Paris Diderot - Paris 7 , Bâtiment Sophie-Germain, 75205 Paris Cedex 13, France; e-mail: leautaud@math.univ-paris-diderot.fr

L. Robbiano: Université de Versailles Saint-Quentin, Laboratoire de Mathématiques de Versailles, CNRS UMR 8100, 45 Avenue des États-Unis, 78035 Versailles, France; e-mail: luc.robbiano@uvsq.fr

Mathematics Subject Classification (2010): 35J15, 35J57, 35S15, 35K05, 93B05 
3.1. Properties of weight functions . . . . . . . . . . . . . . 1505

3.2. A system formulation . . . . . . . . . . . . . . . . . . . . . . . . 1506

3.3. Conjugation by a weight function . . . . . . . . . . . . . . . . 1506

3.4. Phase-space regions . . . . . . . . . . . . . . . . . . . . . . . . 1508

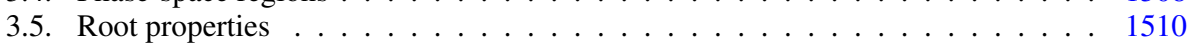

3.6. Microlocalization operators . . . . . . . . . . . . . . . . . . . . . 1512

4. Proof of the Carleman estimate in a neighborhood of the interface . . . . . . . . . . 1513

4.1. Preliminary observations . . . . . . . . . . . . . . . . . . . . . . . 1514

4.2. Estimate in the region $\mathscr{G} \ldots \ldots \ldots \ldots \ldots \ldots \ldots 15 \ldots \ldots \ldots \ldots$

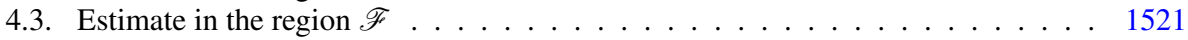

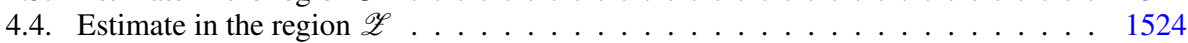

4.5. Estimate in the region $\mathscr{E} \ldots \ldots \ldots \ldots$

4.6. A semiglobal Carleman estimate: proof of Theorem $1.2 \ldots \ldots \ldots$. . . . . . . 1533

5. Interpolation and spectral inequalities . . . . . . . . . . . . . . . 1536

5.1. Interpolation inequality . . . . . . . . . . . . . . . . . . . . . . 1536

5.2. Spectral inequality . . . . . . . . . . . . . . . . . . 1541

References . . . . . . . . . . . . . . . . . . . . . . 1572

\section{Introduction}

When considering elliptic and parabolic operators in $\mathbb{R}^{n}$ with a diffusion coefficient that jumps across an interface of codimension one, say $\left\{x_{n}=0\right\}$, we can interpret the associated equations as two equations with solutions that are coupled at the interface via transmission conditions at $x_{n}=0$, viz. in the parabolic case,

$$
\partial_{t} y_{1}-\nabla_{x} c_{1} \nabla_{x} y_{1}=f_{1} \text { in }\left\{x_{n}<0\right\}, \quad \partial_{t} y_{2}-\nabla_{x} c_{2} \nabla_{x} y_{2}=f_{2} \text { in }\left\{x_{n}>0\right\},
$$

and

$$
y_{1 \mid x_{n}=0^{-}}=y_{2 \mid x_{n}=0^{+}}, \quad c_{1} \partial_{x_{n}} y_{1 \mid x_{n}=0^{-}}=c_{2} \partial_{x_{n}} y_{2 \mid x_{n}=0^{+}} .
$$

Here, we are interested in parabolic/elliptic models in which part of the diffusion occurs along the interface. Then the transmission conditions are of higher order, involving differentiations in the direction of the interface. Such a model can be viewed as an idealization of two diffusive media separated by a thin membrane. We derive this model starting from three media and formally letting the thickness of the intermediate layer become very small. We introduce a small parameter $\delta>0$ that measures the thickness of this layer. Questions such as unique continuation, observation and controllability are natural for such a model. This is the main goal of the present article.

Most of the analysis that we shall carry out concerns a related elliptic operator, including an additional variable. Our key result is the derivation of a Carleman estimate for this operator (see Theorem 1.2 below). The general form of Carleman estimates for a second-order elliptic operator $P$ is (local form)

$$
h\left\|e^{\varphi / h} w\right\|_{L^{2}}^{2}+h^{3}\left\|e^{\varphi / h} \nabla w\right\|_{L^{2}}^{2} \leq C h^{4}\left\|e^{\varphi / h} P w\right\|_{L^{2}}^{2},
$$

for $h$ sufficiently small, an appropriately chosen weight function $\varphi$, and for smooth compactly supported functions $w$. We then deduce an interpolation inequality and a spectral inequality for the original operator in the spirit of the work [LR95]. This spectral inequality then yields the null controllability of the parabolic system considered. An important feature of the results we obtain here is their uniformity in the thickness parameter $\delta$. In 
particular this allows us to recover the earlier results obtained on (1.1)-(1.2) in [LR10]; this corresponds to the limit $\delta \rightarrow 0$ in the model we consider here.

\subsection{Setting}

Let $(\Omega, g)$ be a smooth compact $n$-dimensional $(n \geq 2)$ connected Riemannian manifold (with or without boundary), with $g$ denoting the metric, and $S$ an $n$-1-dimensional smooth submanifold of $\Omega$ (without boundary). We assume ${ }^{1}$ that $\Omega \backslash S=\Omega_{1} \cup \Omega_{2}$ with $\Omega_{1} \cap \Omega_{2}=\emptyset$, so that $\Omega_{1}$ and $\Omega_{2}$ are two smooth open subsets of $\Omega$. Endowed with the metric $g_{\mid T(S)}, S$ has a Riemannian structure. We denote by $\partial_{\eta}$ a nonvanishing vector field defined in a neighborhood of $S$ and normal to $S$ (for the Riemannian metric). We choose the vector field $\partial_{\eta}$ outgoing from $\Omega_{1}$, incoming in $\Omega_{2}$. In local coordinates, we have

$$
\partial_{\eta}=\sum_{j} \eta^{j} \partial_{x_{j}} \quad \text { with } \quad \eta^{j}=\lambda \sum_{k} n_{k} g^{j k}, \quad|\eta|_{g}=1,
$$

where $g^{i j} g_{j k}=\delta_{k}^{i}, \lambda^{2}=\left(g^{i j} n_{i} n_{j}\right)^{-1}$, and $n$ is the normal to $S$ for the Euclidean metric in the local coordinates, outgoing from $\Omega_{1}$, incoming in $\Omega_{2}$. In fact $\lambda_{\mid S}^{2}=\operatorname{det}(g) / \operatorname{det}\left(g_{\mid T(S)}\right)$ at $S$.

The covariant gradient and the divergence operators are given in local coordinates by

$$
\nabla_{g}=\sum_{i} g^{i j} \partial_{x_{i}}, \quad \operatorname{div}_{g} v=\frac{1}{\sqrt{\operatorname{det}(g)}} \sum_{i} \partial_{x_{i}}\left(\sqrt{\operatorname{det}(g)} v_{i}\right),
$$

with similar definition for the gradient $\nabla^{s}=\nabla_{g_{\mid T(S)}}$ and divergence $\operatorname{div}^{s}=\operatorname{div}_{g_{\mid T(S)}}$ on the interface $S$ with the metric $g_{\mid T(S)}$.

We consider a (scalar) diffusion coefficient $c(x)$ with $c_{\mid \Omega_{i}} \in \mathscr{C}^{\infty}\left(\overline{\Omega_{i}}\right), i=1$, 2, yet discontinuous across $S$ and satisfying $c(x) \geq c_{\min }>0$ uniformly for $x \in \Omega_{1} \cup \Omega_{2}$. We set

$$
\Delta_{c}=\operatorname{div}_{g} c(x) \nabla_{g}=\frac{1}{\sqrt{\operatorname{det}(g)}} \sum_{i, j} \partial_{x_{i}}\left(c g^{i j} \sqrt{\operatorname{det}(g)} \partial_{x_{j}}\right) \quad \text { in } \Omega_{1} \cup \Omega_{2},
$$

in local coordinates. Let us denote by $c^{s}$ a smooth (scalar) diffusion coefficient on $S$ satisfying $c^{s}(x) \geq c_{\min }^{s}>0$. Similarly we define $\Delta_{c^{s}}=\operatorname{div}^{s} c^{s} \nabla^{s}$ as a second-order elliptic differential operator on $S$.

In what follows, we shall use the notation $z_{\mid S_{j}}=\left(z_{\mid \Omega_{j}}\right)_{\mid S}, j=1,2$, for the traces of functions on $S$.

Given a time $T>0$, we consider the parabolic control problem

$$
\begin{cases}\partial_{t} z-\Delta_{c} z=\mathbb{1}_{\omega} u & \text { in }(0, T) \times \Omega_{1} \cup \Omega_{2}, \\ \partial_{t} z^{s}-\Delta_{c^{s}} z^{s}=\frac{1}{\delta}\left(\left(c \partial_{\eta} z\right)_{\mid S_{2}}-\left(c \partial_{\eta} z\right)_{\mid S_{1}}\right) & \text { in }(0, T) \times S, \\ z_{\mid S_{1}}=z^{s}=z_{\mid S_{2}} & \text { in }(0, T) \times S, \\ z_{\mid \partial \Omega}=0, & \end{cases}
$$

with some initial data in $L^{2}\left(\Omega_{1} \cup \Omega_{2}\right) \times L^{2}(S)$. Here, $\delta$ denotes a bounded parameter,

\footnotetext{
1 Other geometrical situations can also be dealt with because of the local nature of the estimates we prove here. See Section 1.3.2 below.
} 
$0<\delta \leq \delta_{0}$, and $\omega$ is an open nonempty subset of $\Omega_{1} \cup \Omega_{2}$. Let us suppose for instance that $\omega \subset \Omega_{2}$. The function $u$ is a control function and the null controllability problem concerns the ability to drive the solution $\left(z, z^{S}\right)$ to zero at the final time $T$.

Such a coupling condition at the interface was considered in [KZ06] and [LZ12] for the associated hyperbolic system. In Appendix A, we briefly explain how this model can be formally derived. This model corresponds to two diffusive media separated by a thin layer in which diffusion also occurs. The parameter $\delta$ is then a measure of the thickness of this intermediate layer. In the derivation of the model $\delta$ is assumed to be small.

We present here some function spaces and operators and their basic properties, in order to formulate Problem (1.4) in a more abstract way. The reader is referred to Section 2 for the details. We introduce the Hilbert space $\mathcal{H}_{\delta}^{0}=L^{2}\left(\Omega_{1} \cup \Omega_{2}\right) \times L^{2}(S)$ with the inner product

$$
(Z, \tilde{Z})_{\mathcal{H}_{\delta}^{0}}=(z, \tilde{z})_{L^{2}\left(\Omega_{1} \cup \Omega_{2}\right)}+\delta\left(z^{s}, \tilde{z}^{s}\right)_{L^{2}(S)}, \quad Z=\left(z, z^{s}\right), \tilde{Z}=\left(\tilde{z}, \tilde{z}^{s}\right),
$$

where

$$
(z, \tilde{z})_{L^{2}\left(\Omega_{1} \cup \Omega_{2}\right)}=\int_{\Omega_{1} \cup \Omega_{2}} z \overline{\tilde{z}} d \nu, \quad\left(z^{s}, \tilde{z}^{s}\right)_{L^{2}(S)}=\int_{S} z^{s} \overline{\tilde{z}^{s}} d \nu^{s},
$$

with $d v=\sqrt{\operatorname{det}(g)} d x$ and $d v^{s}=\sqrt{\operatorname{det}\left(g_{\mid T(S)}\right)} d y$. We also introduce the following Hilbert space

$$
\mathcal{H}_{\delta}^{1}=\left\{Z=\left(z, z^{s}\right) \in H^{1}\left(\Omega_{1} \cup \Omega_{2}\right) \times H^{1}(S) ; z_{\mid \partial \Omega}=0 ; z_{\mid S_{1}}=z^{s}=z_{\mid S_{2}}\right\},
$$

with the inner product

$$
\begin{aligned}
(Z, \tilde{Z})_{\mathcal{H}_{\delta}^{1}}= & (Z, \tilde{Z})_{\mathcal{H}_{\delta}^{0}}+\left(c \nabla_{g} z, \nabla_{g} \tilde{z}\right)_{L^{2}\left(\Omega_{1} \cup \Omega_{2}\right)} \\
& +\delta\left(c^{s} \nabla^{s} z, \nabla^{s} \tilde{z}^{s}\right)_{L^{2}(S)}, \quad Z=\left(z, z^{s}\right), \tilde{Z}=\left(\tilde{z}, \tilde{z}^{s}\right) .
\end{aligned}
$$

Problem (1.4) can be written as

$$
\partial_{t} Z+A_{\delta} Z=B u
$$

where the state is $Z=\left(z, z^{s}\right) \in \mathcal{H}_{\delta}^{0}$ and the operator $A_{\delta}$ reads

$$
A_{\delta} Z=\left(\begin{array}{c}
-\Delta_{c} z \\
-\Delta_{c^{s}} z^{s}-\frac{1}{\delta}\left(\left(c \partial_{\eta} z\right)_{\mid S_{2}}-\left(c \partial_{\eta} z\right)_{\mid S_{1}}\right)
\end{array}\right),
$$

with domain

$$
D\left(A_{\delta}\right)=\left\{\left(z, z^{s}\right) \in \mathcal{H}_{\delta}^{1} ; A_{\delta}\left(z, z^{s}\right) \in \mathcal{H}_{\delta}^{0}\right\} .
$$

The operator $\left(A_{\delta}, D\left(A_{\delta}\right)\right)$ is nonnegative self-adjoint on $\mathcal{H}_{\delta}^{0}$. The control operator $B$ is the bounded operator from $L^{2}\left(\Omega_{1} \cup \Omega_{2}\right)$ into $L^{2}\left(\Omega_{1} \cup \Omega_{2}\right) \times L^{2}(S)$ given by $B: u \mapsto$ ${ }^{t}\left(\mathbb{1}_{\omega} u, 0\right)$. We shall prove that System (1.7), i.e. System (1.4), is well-posed for an initial condition in $\mathcal{H}_{\delta}^{0}$.

Remark 1.1. In the limit $\delta \rightarrow 0$, from System (1.4), we obtain the following system (see Section 2.2 for a proof of convergence):

$$
\begin{cases}\partial_{t} z-\Delta_{c} z=\mathbb{1}_{\omega} u & \text { in }(0, T) \times \Omega_{1} \cup \Omega_{2}, \\ \left(c \partial_{\eta} z\right)_{\mid S_{2}}=\left(c \partial_{\eta} z\right)_{\mid S_{1}} \text { and } z_{\mid S_{1}}=z_{\mid S_{2}} & \text { in }(0, T) \times S, \\ z \mid \partial \Omega=0, & \end{cases}
$$


which corresponds to the case studied in [LR10]. We also refer to the recent works [DOP02, BDL07a, Le 07, BDL07b, BGL07, LR11, LL11, BDL11] for the derivation of Carleman estimates for elliptic and parabolic operators with such coefficients with applications to controllability and inverse problems.

\subsection{Statement of the main results}

1.2.1. Carleman estimate. The Carleman estimate we prove concerns an augmented elliptic operator: we introduce an additional coordinate, $x_{0} \in\left(0, X_{0}\right) \subset \mathbb{R}$, so that $\left(x_{0}, x\right) \in$ $\left(0, X_{0}\right) \times \Omega$. This variable $x_{0}$ was introduced in [LR95]; there it allowed us to obtain the null controllability of the heat equation. This approach was followed in several works [LZ98, JL99, LR10]. It was also used to prove stabilization properties of the wave equation [Leb96, LR97].

We consider the $n+1$-dimensional partially determined elliptic problem

$$
\begin{cases}-\partial_{x_{0}}^{2} w-\Delta_{c} w+\nabla_{a} w+b w=f & \text { in }\left(0, X_{0}\right) \times\left(\Omega_{1} \cup \Omega_{2}\right), \\ -\partial_{x_{0}}^{2} w^{s}-\Delta_{c} w^{s}+\nabla_{a}^{s} w^{s}+b^{s} w^{s} & \\ \quad=\frac{1}{\delta}\left(\left(c \partial_{\eta} w\right)_{\mid\left(0, X_{0}\right) \times S_{2}}-\left(c \partial_{\eta} w\right)_{\mid\left(0, X_{0}\right) \times S_{1}}+\theta^{s}\right) & \text { in }\left(0, X_{0}\right) \times S \\ w_{\mid\left(0, X_{0}\right) \times S_{1}=w^{s}+\theta^{1}} \text { and } w_{\mid\left(0, X_{0}\right) \times S_{2}}=w^{s}+\theta^{2} & \text { in }\left(0, X_{0}\right) \times S .\end{cases}
$$

Note that we add lower-order terms to the elliptic operators here: $\nabla_{a}$ (resp. $\nabla_{a}^{s}$ ) denotes any smooth vector field on $\Omega_{1} \cup \Omega_{2}$ (resp. $S$ ) and $b$ (resp. $b^{s}$ ) some bounded function on $\Omega_{1} \cup \Omega_{2}$ (resp. $S$ ). Moreover, we include source terms $\theta^{j}, j=1,2, \theta^{s}$ at the interface through the transmission conditions. This system is not fully determined as we do not prescribe any boundary condition on $\{0\} \times \Omega$ and $\left\{X_{0}\right\} \times \Omega$.

In Section 3, we introduce a small neighborhood $V_{\varepsilon}$ of $S$ in $\Omega$, where we can use coordinates of the form $\left(y, x_{n}\right)$ with $y \in S$ and $x_{n} \in[-2 \varepsilon, 2 \varepsilon]$. We then set $\mathcal{M}=$ $\left(0, X_{0}\right) \times V_{\varepsilon}$ and $\mathcal{M}_{j}=\mathcal{M} \cap\left(\left(0, X_{0}\right) \times \Omega_{j}\right), j=1,2$.

For a properly chosen weight function $\varphi$ (see Section 3.1), for some $0<\alpha_{0}<X_{0} / 2$, and a cut-off function $\zeta=\zeta\left(x_{n}\right) \in \mathscr{C}_{c}^{\infty}([0,2 \varepsilon))$, with $\zeta=1$ on $[0, \varepsilon)$, we shall prove the following theorem.

Theorem 1.2. For all $\delta_{0}>0$, there exist $C>0$ and $h_{0}>0$ such that

$$
\begin{aligned}
& h\left\|e^{\varphi / h} w\right\|_{0}^{2}+h^{3}\left\|e^{\varphi / h} \nabla_{x_{0}, x} w\right\|_{0}^{2}+h \sum_{j=1,2}\left|e^{\varphi / h} w_{\mid S_{j}}\right|_{0}^{2}+h^{3} \sum_{j=1,2}\left|e^{\varphi / h} \nabla_{x_{0}, x} w_{\mid S_{j}}\right|_{0}^{2} \\
& \leq C\left(h^{4}\left\|e^{\varphi / h} f_{\mid \mathcal{M}_{1}}\right\|_{0}^{2}+h^{4}\left\|e^{\varphi / h} f_{\mid \mathcal{M}_{2}}\right\|_{0}^{2}+h^{2} \delta^{2}\left\|\zeta e^{\varphi / h} f_{\mid \mathcal{M}_{2}}\right\|_{0}^{2}+h\left|e^{\varphi / h} \theta^{1}\right|_{0}^{2}\right. \\
& \left.\quad+\left(h+\delta^{2} / h\right)\left|e^{\varphi / h} \theta^{2}\right|_{0}^{2}+h^{3}\left|e^{\varphi / h} \nabla_{x_{0}, S} \theta^{1}\right|_{0}^{2}+h^{3}\left|e^{\varphi / h} \nabla_{x_{0}, S} \theta^{2}\right|_{0}^{2}+h^{3}\left|e^{\varphi / h} \theta^{s}\right|_{0}^{2}\right)
\end{aligned}
$$

for all $0<\delta<\delta_{0}, 0<h \leq h_{0}$, for $\left(w, \theta^{1}, \theta^{2}, \theta^{s}, f\right)$ satisfying (1.11), $w_{\mid \mathcal{M}_{j}} \in$ $\mathscr{C}^{\infty}\left(\overline{\mathcal{M}_{j}}\right)$, and $w^{s} \in \mathscr{C}^{\infty}\left(\left(0, X_{0}\right) \times S\right)$ with

$$
\operatorname{supp}(w) \subset\left(\alpha_{0}, X_{0}-\alpha_{0}\right) \times S \times(-2 \varepsilon, 2 \varepsilon), \quad \operatorname{supp}\left(w^{s}\right) \subset\left(\alpha_{0}, X_{0}-\alpha_{0}\right) \times S .
$$


Here $\nabla_{x_{0}, x}=\left(\partial_{x_{0}}, \nabla_{g}\right)^{t}, \nabla_{x_{0}, S}=\left(\partial_{x_{0}}, \nabla^{s}\right)^{t}$ and $\|\cdot\|_{0},|\cdot|_{0}$ are $L^{2}$ norms on $\mathcal{M}$ and $\left(0, X_{0}\right) \times S$ respectively. The weight function $\varphi$ will be chosen increasing when crossing $S$ from $\mathcal{M}_{1}$ to $\mathcal{M}_{2}$, which corresponds to an observation on the side $\left(0, X_{0}\right) \times \Omega_{2}$. Observe the nonsymmetric form of the r.h.s. of the estimate above. This originates from our choice of observing the solution $w$ in $\left(0, X_{0}\right) \times \Omega_{2}$.

This type of Carleman estimate is well known away from the interface $S$ (see [Hör63], and [LR95] for an estimate at the Dirichlet boundary $\partial \Omega$ ).

Remark 1.3. The additional variable $x_{0}$ is used here to obtain the spectral inequality of Theorem 1.5 below. The same Carleman inequality holds for the operator $A_{\delta}$. The proof can be adapted from that of Theorem 1.2. In fact, without the additional variable, the proof becomes less involved.

The Carleman estimate of Theorem 1.2 exhibits the loss of half a derivative apart from one term on the r.h.s. (see below). Usually, one proves such Carleman estimates locally in a neighborhood of a point, for instance using local coordinates, treating only the principal part of the operator. Next, one includes lower order terms in the operator, exploiting that the associated contributions can be absorbed thanks to the coefficients $h^{\alpha}$ of the terms on the 1.h.s. of the Carleman estimate. ${ }^{2}$ Finally, one patches these estimates together if a global estimate is needed. This can be achieved again thanks to the precise powers of $h$ in all the terms. For a review of these derivations see for example [LL12].

At the interface, for technical reasons, in the following terms on the r.h.s. of (1.12):

$$
h^{2} \delta^{2}\left\|\zeta e^{\varphi / h} f_{\left|\mathcal{M}_{2}\right|}\right\|_{0}^{2}+\frac{\delta^{2}}{h}\left|e^{\varphi / h} \theta^{2}\right|_{0}^{2}
$$

the powers of $h$ are not the usual expected ones: $h^{4}$ for the first term and $h$ for the second one. For the first term this corresponds in fact to a loss of one and a half derivative. We do not know if these two terms are optimal or not. If we simply prove the Carleman estimate in the neighborhood of a point, because of the powers of $h$ in these terms such local estimates cannot be patched together. The obstruction originates from the diffusion that occurs in the $(n-1)$-dimensional submanifold $S$ through the operator $\Delta_{c^{s}}$. Note that this obstruction naturally disappears in the limit $\delta \rightarrow 0$.

Our strategy will thus differ from what is done classically. The estimate of Theorem 1.2 is of semiglobal nature. It is global in the direction of the submanifold $S$ and local in the other directions ( $x_{0}$ and a normal direction to $S$ in $\Omega$ ): we work in a neighborhood of the whole interface $S$. Thanks to the cut-off function $\zeta$ that confines the term

$$
h^{2} \delta^{2}\left\|\zeta e^{\varphi / h} f_{\mid \mathcal{M}_{2}}\right\|_{0}^{2}
$$

to a neighborhood of $S$, estimate (1.12) can in turn be patched with Carleman estimates away from the interface to form a global estimate. Moreover for the same reasons we do

\footnotetext{
2 Note that the powers of $h$ in estimate (1.3) are in fact optimal.
} 
not restrict our analysis to the principal part: in the proof we also consider the first-order terms of the operator. ${ }^{3}$

Following [LR10] we shall introduce microlocal regions. Here, the regions are defined on the whole (cotangent bundle of $S$. For each region we shall derive a partial Carleman estimate. The different estimates can then be patched together to yield (1.12). Our strategy requires us to work on $S$ globally; we shall thus consider (pseudo-)differential operators on $S$. Yet, we shall often use their expression in local coordinates; this will allow us to use some of the results proven in [LR10].

For the purpose of proving the null controllability of the parabolic problem (1.4), a local Carleman estimate of the form of Theorem 1.2 in the neighborhood of any point at the interface would be sufficient. Yet, an important property of Carleman estimates resides in the possibility of patching them together to obtain a global estimate. Our result thus preserves this important feature.

1.2.2. Interpolation inequality. With the Carleman estimate of Theorem 1.2 we then prove an interpolation inequality of the form introduced in [LR95]. This type of interpolation inequality for elliptic operators has also been used in [Leb96, LR97] to address stabilization problems for the wave equation.

Let $\alpha_{1} \in\left[0, X_{0} / 2\right)$. We set $\mathcal{K}_{\delta}^{0}\left(\alpha_{1}\right)=L^{2}\left(\left(\alpha_{1}, X_{0}-\alpha_{1}\right) ; \mathcal{H}_{\delta}^{0}\right)$ with $\mathcal{K}_{\delta}^{0}=\mathcal{K}_{\delta}^{0}(0)$, and we define the following Sobolev spaces:

$$
\begin{aligned}
\mathcal{K}_{\delta}^{1}\left(\alpha_{1}\right) & =L^{2}\left(\left(\alpha_{1}, X_{0}-\alpha_{1}\right) ; \mathcal{H}_{\delta}^{1}\right) \cap H^{1}\left(\left(\alpha_{1}, X_{0}-\alpha_{1}\right) ; \mathcal{H}_{\delta}^{0}\right), \quad \mathcal{K}_{\delta}^{1}=\mathcal{K}_{\delta}^{1}(0), \\
\mathcal{K}_{\delta}^{2} & =L^{2}\left(\left(0, X_{0}\right) ; D\left(A_{\delta}\right)\right) \cap H^{1}\left(\left(0, X_{0}\right) ; \mathcal{H}_{\delta}^{1}\right) \cap H^{2}\left(\left(0, X_{0}\right) ; \mathcal{H}_{\delta}^{0}\right) .
\end{aligned}
$$

Theorem 1.4. For all $\delta_{0}>0$, there exist $C \geq 0$ and $\nu_{0} \in(0,1)$ such that for all $\delta \in$ $\left(0, \delta_{0}\right)$,

$$
\|U\|_{\mathcal{K}_{\delta}^{1}\left(\alpha_{1}\right)} \leq C\|U\|_{\mathcal{K}_{\delta}^{1}}^{1-\nu_{0}}\left(\left\|\left(-\partial_{x_{0}}^{2}+A_{\delta}\right) U\right\|_{\mathcal{K}_{\delta}^{0}}+\left\|\partial_{x_{0}} u(0, x)\right\|_{L^{2}(\omega)}\right)^{\nu_{0}}
$$

for all $U=\left(u, u^{s}\right) \in \mathcal{K}_{\delta}^{2}$ with $u_{\mid x_{0}=0}=0$ in $\Omega_{1} \cup \Omega_{2}$.

An important consequence of this interpolation inequality is the spectral inequality that we present in the next section.

1.2.3. Spectral inequality and null controllability result. From the above interpolation inequality we deduce a spectral inequality for the elliptic operator $A_{\delta}$ defined in (1.8). We consider $\mathscr{E}_{\delta, j}=\left(e_{\delta, j}, e_{\delta, j}^{s}\right), j \in \mathbb{N}$, a Hilbert basis of $\mathcal{H}_{\delta}^{0}$ composed of eigenfunctions of the operator $A_{\delta}$ associated with the nonnegative eigenvalues $\mu_{\delta, j} \in \mathbb{R}, j \in \mathbb{N}$, sorted in an increasing sequence (see Proposition 2.5).

Theorem 1.5. For $\delta_{0}>0$, there exists $C>0$ such that for all $0<\delta \leq \delta_{0}$ and $\mu \in \mathbb{R}$,

$$
\|Z\|_{\mathcal{H}_{\delta}^{0}} \leq C e^{C \sqrt{\mu}}\|z\|_{L^{2}(\omega)}, \quad Z=\left(z, z^{s}\right) \in \operatorname{span}\left\{\mathscr{E}_{\delta, j} ; \mu_{\delta, j} \leq \mu\right\}
$$

Following [LR95], this estimate then yields a construction of the control function $u_{\delta}(t, x)$ in (1.4), by sequentially acting on a finite yet increasing number of eigenspaces, and we

\footnotetext{
3 This technical point explains the regularity requirements we made above for $\nabla_{a}$ and $\nabla_{a}^{s}$. Yet, we can treat bounded coefficients for the zero-order terms.
} 
hence obtain the following $\delta$-uniform controllability theorem. The proof can be adapted from those in [LR95] or [LZ98, Section 5, Proposition 2], and the uniformity with respect to the parameter $\delta>0$ comes naturally. We also refer to [LL12] for an exposition of the method and to [Mil06, Léa10, Mil10, TT11] for further developments.

Theorem 1.6. Let $\delta_{0}>0$. For an arbitrary time $T>0$ and an arbitrary nonempty open subset $\omega \subset \Omega$ there exists $C>0$ such that: for all initial conditions $Z_{0}=\left(z_{0}, z_{0}^{s}\right) \in \mathcal{H}_{\delta}^{0}$ and all $0<\delta \leq \delta_{0}$, there exists $u_{\delta} \in L^{2}((0, T) \times \omega)$ such that the solution $\left(z, z^{s}\right)$ of (1.4) satisfies $\left(z(T), z^{s}(T)\right)=(0,0)$ and moreover

$$
\left\|u_{\delta}\right\|_{L^{2}((0, T) \times \omega)} \leq C\left\|Z_{0}\right\|_{\mathcal{H}_{\delta}^{0}} .
$$

An important feature of this result is that the control is uniformly bounded as $\delta \rightarrow 0$, so that we can extract a subsequence $u_{\delta}$ weakly convergent in $L^{2}((0, T) \times \omega)$. In Corollary 2.9 below we prove that the associated solution of Problem (1.4) converges towards a controlled solution of Problem (1.10). For this last control problem (previously treated in [LR10]), we hence construct a control function which is robust with respect to small viscous perturbations in the interface.

It is classical to deduce a boundary null controllability result from the previous distributed control result.

N.B. Here, for the sake of fixing the notation for the statement of the Carleman estimate above, we chose the observation in $\Omega_{2}$. This corresponds to $\omega \subset \Omega_{2}$ in the proofs of Theorems 1.4-1.6. Yet, $\omega$ can be chosen as an arbitrary open subset of $\Omega$.

\subsection{Some additional results and remarks}

1.3.1. A stabilization result.. A second important consequence of the interpolation inequality of Theorem 1.4 concerns the stabilization properties of the hyperbolic system (studied in [KZ06, LZ12])

$$
\begin{cases}\partial_{t t} z-\Delta_{c} z+a(x) \partial_{t} z=0 & \text { in }(0, T) \times \Omega_{1} \cup \Omega_{2}, \\ \partial_{t t} z^{s}-\Delta_{c} z^{s}=\frac{1}{\delta}\left(\left(c \partial_{\eta} z\right)_{\mid S_{2}}-\left(c \partial_{\eta} z\right)_{\mid S_{1}}\right) & \text { in }(0, T) \times S, \\ z_{\mid S_{1}}=z^{s}=z_{\mid S_{2}} & \text { in }(0, T) \times S, \\ z_{\mid \partial \Omega}=0, & \end{cases}
$$

where $a$ is a nonvanishing nonnegative smooth function on $\Omega_{1} \cup \Omega_{2}$. According to [Leb96, LR97], a local version of (1.13) (see Lemma 5.1 below) allows one to produce resolvent estimates which in turn give a result of the following type: for all $\delta_{0}>0$ and all $k \in \mathbb{N}$ there exists $C>0$ such that for any $0<\delta<\delta_{0}$, we have the energy decay estimate

$$
\begin{aligned}
\left\|\left(\partial_{t} z, \partial_{t} z^{s}\right)\right\|_{\mathcal{H}_{\delta}^{0}}+\| & \left(z, z^{s}\right) \|_{\mathcal{H}_{\delta}^{1}} \\
& \leq \frac{C}{[\log (2+t)]^{k}}\left(\left\|\left(\partial_{t} z, \partial_{t} z^{s}\right)_{\mid t=0}\right\|_{D\left(A_{\delta}^{k / 2}\right)}+\left\|\left(z, z^{s}\right)_{\mid t=0}\right\|_{D\left(A_{\delta}^{(k+1) / 2}\right)}\right)
\end{aligned}
$$

for all solutions of (1.15). In particular, this decay rate is uniform with respect to $\delta$. See [Bur98, Theorem 3] to obtain the power $k$ exactly. The same properties can be obtained for this hyperbolic system with a boundary damping (see [LR97]). 
1.3.2. Other geometrical situations. Above we assumed that $\Omega$ could be partitioned as $\Omega=\Omega_{1} \cup \Omega_{2} \cup S$. More general situations can be treated (interpolation and spectral inequalities, and null controllability result) because of the local nature of the Carleman estimate of Theorem 1.2. If $V$ is a neighborhood of $S$, we require $V$ to be of the form $V_{1} \cup V_{2} \cup S$ with $V_{1}$ and $V_{2}$ on both sides of $S$. Several nonintersecting interfaces can be considered as well. For example, the geometrical situations in Figure 1 can be addressed as well. If needed, a global Carleman estimate can be derived by combining Theorem 1.2 and the arguments of Section 5 in [LR11].

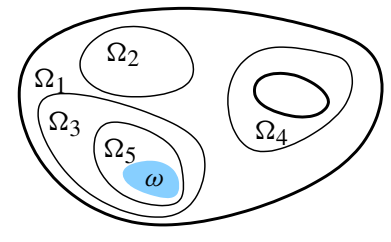

(a)

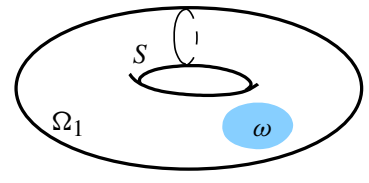

(b)

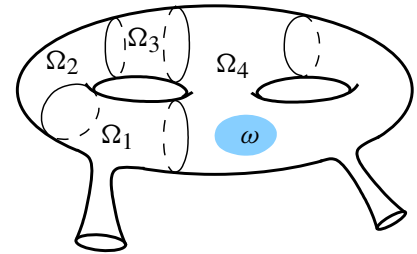

(c)

Fig. 1. Other geometrical situations: (a) $\Omega$ is a bounded open subset of $\mathbb{R}^{n}$; (b) and (c) $\Omega$ is a compact manifold with boundary.

1.3.3. Lack of controllability from the interface. It is important to note that the parabolic controllability result of Theorem 1.6 does not hold in general if the control function acts on the interface $S$. Let $\omega^{s}$ be an open subset of $S$. Then in general there is no $u \in L^{2}((0, T) \times S)$ that brings the solution of

$$
\begin{cases}\partial_{t} z-\Delta_{c} z=0 & \text { in }(0, T) \times \Omega_{1} \cup \Omega_{2}, \\ \partial_{t} z^{s}-\Delta_{c^{s}} z^{s}=\frac{1}{\delta}\left(\left(c \partial_{\eta} z\right)_{\mid S_{2}}-\left(c \partial_{\eta} z\right)_{\mid S_{1}}\right)+\mathbb{1}_{\omega^{s}} u & \text { in }(0, T) \times S, \\ z_{\mid S_{1}}=z^{s}=z_{\mid S_{2}} & \text { in }(0, T) \times S, \\ z_{\mid \partial \Omega}=0 & \end{cases}
$$

to zero at time $T$.

Let us consider the following two-dimensional example: $\Omega=\mathbb{R} /(2 \pi \mathbb{Z}) \times(-\pi, \pi)$ is the cylinder endowed with a flat metric. For consistency with the notation of Section 3 we use $\left(y, x_{n}\right)$ as the coordinates in $\Omega$, with periodic conditions in $y$. We define the interface as $S=\left\{x_{n}=0\right\}=\mathbb{R} /(2 \pi \mathbb{Z}) \times\{0\}$, so that $\Omega_{1}=\left\{x_{n}<0\right\}$ and $\Omega_{2}=\left\{x_{n}>0\right\}$.

We take the diffusion coefficient $c$ to be piecewise constant (i.e. $c=c_{j}$ in $\Omega_{j}$ for $j=1,2$ ) and define the operator $A_{\delta}$ as in (1.8) (with Dirichlet boundary conditions in the $x_{n}$ variable). In this geometrical context, we have the following result.

Proposition 1.7. If $\gamma:=\sqrt{c_{2} / c_{1}} \in \mathbb{N}^{*}$, then for all $c^{s}>0, \delta>0$, and $j \in \mathbb{Z}$, the function

$$
\mathscr{E}_{\delta, j}:=\left(\begin{array}{c}
e_{\delta, j} \\
0
\end{array}\right) \quad \text { with } \quad e_{\delta, j}\left(y, x_{n}\right)= \begin{cases}e^{i j \gamma y} \sin \left(\gamma^{2} j x_{n}\right) & \text { for } x_{n}<0, \\
e^{i j \gamma y} \sin \left(j x_{n}\right) & \text { for } x_{n}>0,\end{cases}
$$

is an eigenfunction of the operator $A_{\delta}$ associated with the eigenvalue $c_{2} j^{2}\left(1+\gamma^{2}\right)$. 
As a consequence, the adjoint problem of (1.16) (which is of the same form as (1.16) without any control function) does not have the unique continuation property when observed from any subset of $S$. More precisely, we notice that the set of "invisible" modes is of infinite dimension. As a consequence, System (1.16) is not approximately controllable in this case and moreover the set of noncontrollable modes is of infinite dimension.

The phenomenon exhibited in this example is due to the high level of symmetry. However, in a general setting, if the Laplace operator has an eigenfunction which has a $\mathscr{C}^{\infty}$ closed nodal curve, then the associated problem (1.16) with $c_{1}=c_{2}=1$ and $S$ given by this nodal curve is not controllable from $S$. We hence see that this question is connected to properties of the eigenfunctions of the Laplace operator and of their nodal sets.

\subsection{Notation: semiclassical operators and geometrical setting}

1.4.1. Semiclassical operators on $\mathbb{R}^{d}$. We shall use the notation $\langle\eta\rangle:=\left(1+|\eta|^{2}\right)^{1 / 2}$. For a parameter $h \in\left(0, h_{0}\right]$ for some $h_{0}>0$, we denote by $S^{m}\left(\mathbb{R}^{d} \times \mathbb{R}^{d}\right), S^{m}$ for short, the space of smooth functions $a(z, \zeta, h)$ that satisfy the following property: for all multi-indices $\alpha, \beta$, there exists $C_{\alpha, \beta} \geq 0$ such that

$$
\left|\partial_{z}^{\alpha} \partial_{\zeta}^{\beta} a(z, \zeta, h)\right| \leq C_{\alpha, \beta}\langle\zeta\rangle^{m-|\beta|}, \quad z \in \mathbb{R}^{d}, \zeta \in \mathbb{R}^{d}, h \in\left(0, h_{0}\right] .
$$

Then, for all sequences $a_{m-j} \in S^{m-j}, j \in \mathbb{N}$, there exists a symbol $a \in S^{m}$ such that $a \sim \sum_{j} h^{j} a_{m-j}$, in the sense that

$$
a-\sum_{j<N} h^{j} a_{m-j} \in h^{N} S^{m-N}
$$

(see for instance [Mar02, Proposition 2.3.2] or [Hör85a, Proposition 18.1.3]), with $a_{m}$ as principal symbol. We define $\Psi^{m}$ as the space of semiclassical operators $A=\operatorname{Op}(a)$, for $a \in S^{m}$, formally defined by

$$
A u(z)=(2 \pi h)^{-d} \iint e^{i\langle z-t, \zeta\rangle / h} a(z, \zeta, h) u(t) d t d \zeta, \quad u \in \mathscr{S}^{\prime}\left(\mathbb{R}^{d}\right) .
$$

We shall denote the principal symbol $a_{m}$ by $\sigma(A)$. We shall use the techniques of pseudodifferential calculus in this article, such as construction of parametrices, composition formula, formula for the symbol of the adjoint operator, etc. We refer the reader to [Tay81, Hör85a, Mar02]. We provide composition and change of variables formulæ in the case of tangential operators in Appendix B. Those formulæ can be adapted to the case of operators acting in the whole space $\mathbb{R}^{d}$. In the main text the variable $z$ will be $\left(x_{0}, x\right) \in \mathbb{R}^{n+1}$ and $\zeta=\left(\xi_{0}, \xi\right) \in \mathbb{R}^{n+1}$.

We set

$$
\begin{aligned}
& S^{-\infty}=\bigcap_{m>0} S^{-m}, \quad h^{\infty} S^{-\infty}=\bigcap_{m>0} h^{m} S^{-m}, \\
& \Psi^{-\infty}=\bigcap_{m>0} \Psi^{-m}, \quad h^{\infty} \Psi^{-\infty}=\bigcap_{m>0} h^{m} \Psi^{-m} .
\end{aligned}
$$


Note that if there exists a closed set $F$ such that in the asymptotic expansion (1.17) we have $\operatorname{supp}\left(a_{m-j}\right) \subset F, j \in \mathbb{N}$, then a representative of $a$ modulo $h^{\infty} S^{-\infty}$ can be chosen supported in $F$.

We shall also denote by $\mathscr{D}^{m}$ the space of semiclassical differential operators, i.e., the case where $a(z, \zeta, h)$ is a polynomial function of order $m$ in $\zeta$. In particular we set

$$
D=\frac{h}{i} \partial, \quad \text { and we have } \quad \sigma(D)=\xi .
$$

We now introduce Sobolev spaces on $\mathbb{R}^{d}$ and Sobolev norms which are adapted to the scaling parameter $h$. The natural norm on $L^{2}\left(\mathbb{R}^{d}\right)$ is written as $\|u\|_{L^{2}\left(\mathbb{R}^{d}\right)}=\|u\|_{0}:=$ $\left(\int|u(x)|^{2} d x\right)^{1 / 2}$. Let $r \in \mathbb{R}$; we then set

$$
\|u\|_{r}=\|u\|_{\mathscr{H}^{r}\left(\mathbb{R}^{d}\right)}=\left\|\Lambda^{r} u\right\|_{0},
$$

with

$$
\Lambda^{r}:=\operatorname{Op}\left(\langle\xi\rangle^{r}\right) \quad \text { and } \quad \mathscr{H}^{r}\left(\mathbb{R}^{d}\right):=\left\{u \in \mathscr{S}^{\prime}\left(\mathbb{R}^{d}\right) ;\|u\|_{r}<\infty\right\} .
$$

The space $\mathscr{H}^{r}\left(\mathbb{R}^{d}\right)$ is algebraically equal to the classical Sobolev space $H^{r}\left(\mathbb{R}^{d}\right)$. For a fixed value of $h$, the norm $\|\cdot\|_{r}$ is equivalent to the classical Sobolev norm that we write $\|\cdot\|_{H^{r}\left(\mathbb{R}^{d}\right)}$. However, these norms are not uniformly equivalent as $h$ goes to 0 .

1.4.2. Tangential semiclassical operators on $\mathbb{R}^{d}, d \geq 2$. We set $z=\left(z^{\prime}, z_{d}\right), z^{\prime}=$ $\left(z_{1}, \ldots, z_{d-1}\right)$ and $\zeta^{\prime}=\left(\zeta_{1}, \ldots, \zeta_{d-1}\right)$ accordingly. We denote by $S_{\mathcal{T}}^{m}\left(\mathbb{R}^{d} \times \mathbb{R}^{d-1}\right)$, $S_{\mathcal{T}}^{m}$ for short, the space of smooth functions $b\left(z, \zeta^{\prime}, h\right)$, defined for $h \in\left(0, h_{0}\right]$ for some $h_{0}>0$, that have the following property: for all multi-indices $\alpha, \beta$, there exists $C_{\alpha, \beta} \geq 0$ such that

$$
\left|\partial_{z}^{\alpha} \partial_{\zeta^{\prime}}^{\beta} b\left(z, \zeta^{\prime}, h\right)\right| \leq C_{\alpha, \beta}\left\langle\zeta^{\prime}\right\rangle^{m-|\beta|}, \quad z \in \mathbb{R}^{d}, \zeta^{\prime} \in \mathbb{R}^{d-1}, h \in\left(0, h_{0}\right]
$$

As above, for any sequence $b_{m-j} \in S_{\mathcal{T}}^{m-j}, j \in \mathbb{N}$, there exists a symbol $b \in S_{\mathcal{T}}^{m}$ such that $b \sim \sum_{j} h^{j} b_{m-j}$, in the sense that $b-\sum_{j<N} h^{j} b_{m-j} \in h^{N} S_{\mathcal{T}}^{m-N}$, with $b_{m}$ as principal symbol. We define $\Psi_{\mathcal{T}}^{m}$ as the space of tangential semiclassical operators $B=\mathrm{Op}_{\mathcal{T}}(b)$ (observe that the notation we adopt is different from that above to avoid confusion), for $b \in S_{\mathcal{T}}^{m}$, formally defined by

$$
B u(z)=(2 \pi h)^{-(d-1)} \iint e^{i\left\langle z^{\prime}-t^{\prime}, \zeta^{\prime}\right\rangle / h} b\left(z, \zeta^{\prime}, h\right) u\left(t^{\prime}, z_{d}\right) d t^{\prime} d \zeta^{\prime}, \quad u \in \mathscr{S}^{\prime}\left(\mathbb{R}^{d}\right) .
$$

In the main text the variable $z$ will be $\left(x_{0}, x^{\prime}, x_{n}\right) \in \mathbb{R}^{n+1}$ and $\zeta^{\prime}=\left(\xi_{0}, \xi^{\prime}\right) \in \mathbb{R}^{n}$.

We shall also denote the principal symbol $b_{m}$ by $\sigma(B)$. In the case where the symbol is polynomial in $\zeta^{\prime}$ and $h$, we shall denote the space of associated tangential differential operators by $\mathscr{D}_{\mathcal{T}}^{m}$. We shall denote by $\Lambda_{\mathcal{T}}^{s}$ the tangential pseudo-differential operator whose symbol is $\left\langle\zeta^{\prime}\right\rangle^{s}$. We set

$$
\begin{aligned}
& S_{\mathcal{T}}^{-\infty}=\bigcap_{m>0} S_{\mathcal{T}}^{-m}, \quad h^{\infty} S_{\mathcal{T}}^{-\infty}=\bigcap_{m>0} h^{m} S_{\mathcal{T}}^{-m}, \\
& \Psi_{\mathcal{T}}^{-\infty}=\bigcap_{m>0} \Psi_{\mathcal{T}}^{-m}, \quad h^{\infty} \Psi_{\mathcal{T}}^{-\infty}=\bigcap_{m>0} h^{m} \Psi_{\mathcal{T}}^{-m} .
\end{aligned}
$$


For functions defined on $z_{d}=0$ or restricted to $z_{d}=0$, following [LR95, LR97], we shall denote by $(\cdot, \cdot)_{0}$ the inner product, i.e., $(f, g)_{0}:=\iint f\left(z^{\prime}\right) \bar{g}\left(z^{\prime}\right) d z^{\prime}$. The induced norm is denoted by $|\cdot|_{0}$, i.e., $|f|_{0}^{2}=(f, f)_{0}$. For $r \in \mathbb{R}$ we introduce

$$
|f|_{\mathscr{H}^{r}\left(\mathbb{R}^{d-1}\right)}=|f|_{r}:=\left|\Lambda_{\mathcal{T}}^{r} f\right|_{0} .
$$

The composition formula and the action of change of variables are given in Appendix B.1.

Note that we shall keep the notation $\Psi_{\mathcal{T}}^{m}$ for operators with symbols independent of $z_{d}$, acting on $\left\{z_{d}=0\right\}$. These operators are in fact in $\Psi^{m}\left(\mathbb{R}^{d-1}\right)$. A similar notation will be used in the case of operators on a manifold.

1.4.3. Local charts, pullbacks, and Sobolev norms. The submanifold $S$ is of dimension $n-1$ and is furnished with a finite atlas $\left(U_{j}, \phi_{j}\right), j \in J$. The map $\phi_{j}: U_{j} \rightarrow \tilde{U}_{j} \subset \mathbb{R}^{n-1}$ is a smooth diffeomorphism. If $U_{j} \cap U_{k} \neq \emptyset$ we also set

$$
\phi_{j k}: \phi_{j}\left(U_{j} \cap U_{k}\right) \subset \tilde{U}_{j} \rightarrow \phi_{k}\left(U_{j} \cap U_{k}\right) \subset \tilde{U}_{k}, \quad y \mapsto \phi_{k} \circ \phi_{j}^{-1}(y) .
$$

The local charts and the diffeomorphisms we introduce are illustrated in Figure 2.

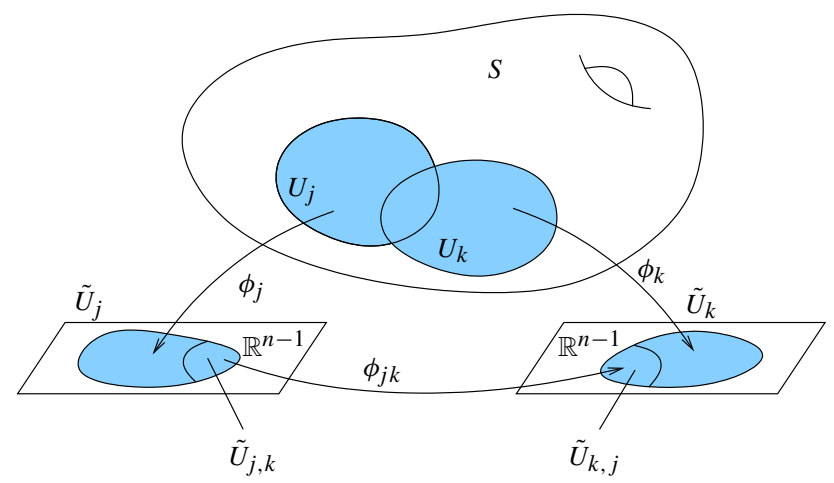

Fig. 2. Local charts and diffeomorphisms for the submanifold $S$.

For a diffeomorphism $\phi$ between two open sets, $\phi: U_{1} \rightarrow U_{2}$, the associated pullback (here stated for continuous functions) is

$$
\phi^{*}: \mathscr{C}\left(U_{2}\right) \rightarrow \mathscr{C}\left(U_{1}\right), \quad u \mapsto u \circ \phi
$$

For a function defined on phase space, e.g. a symbol, the pullback is given by

$$
\phi^{*} u(y, \eta)=u\left(\phi(y),{ }^{t}\left(\phi^{\prime}(y)\right)^{-1} \eta\right), \quad y \in U_{1}, \eta \in T_{y}^{*}\left(U_{1}\right), u \in \mathscr{C}\left(T^{*} U_{2}\right) .
$$

We shall use semiclassical Sobolev norms over the manifold $S$ together with a finite atlas $\left(U_{j}, \phi_{j}\right)_{j}, \phi_{j}: U_{j} \rightarrow \mathbb{R}^{n-1}$, and a partition of unity $\left(\psi_{j}\right)_{j}$ subordinated to this 
covering of $S$ :

$$
\psi_{j} \in \mathscr{C}^{\infty}(S), \quad \operatorname{supp}\left(\psi_{j}\right) \subset U_{j}, \quad 0 \leq \psi_{j} \leq 1, \quad \sum_{j} \psi_{j}=1 .
$$

We then set

$$
|u|_{\mathscr{H}^{r}(S)}=\sum_{j}\left|\left(\phi_{j}^{-1}\right)^{*} \psi_{j} u\right|_{\mathscr{H}^{r}\left(\mathbb{R}^{n-1}\right)} .
$$

Note that the 1.h.s. denotes a norm on the manifold and the r.h.s. is defined in (1.18). We shall need the following elementary result.

Lemma 1.8. Let $\left(f_{j}\right)_{j}$ be a family of smooth functions on $S$ with $\operatorname{supp}\left(f_{j}\right) \subset U_{j}$ and $\sum_{j} f_{j}=f \geq C>0$ in $S$. We set $N_{r}(u)=\sum_{j}\left|\left(\phi_{j}^{-1}\right)^{*} f_{j} u\right|_{\mathscr{C}\left(\mathbb{R}^{n-1}\right)}$. Then $N_{r}$ is a norm equivalent to $|\cdot| \mathscr{H}_{\left(\mathbb{R}^{n-1}\right)}$, uniformly in $h$.

For a proof see Appendix C.1. Note that the $L^{2}$ norm $(r=0)$ defined in (1.20) is equivalent to the natural $L^{2}$ norm on the Riemannian manifold $S$ given through the inner product in (1.5).

Norms in codimension 1. For a function $u$ defined on $\left(0, X_{0}\right) \times \mathbb{R}^{n-1}$ we set

$$
|u|_{0}=|u|_{L^{2}\left(\left(0, X_{0}\right) \times \mathbb{R}^{n-1}\right)}, \quad|u|_{1}^{2}=\left|D_{x_{0}} u\right|_{0}^{2}+\int_{0}^{X_{0}}|u|_{\mathscr{H}^{1}\left(\mathbb{R}^{n-1}\right)}^{2} d x_{0} .
$$

Note that the latter norm is equivalent to $|u|_{\mathscr{H} 1}\left(\mathbb{R} \times \mathbb{R}^{n-1}\right)$ if moreover the function $u$ is compactly supported in the $x_{0}$ variable. For a function $u$ defined on $\left(0, X_{0}\right) \times S$, we set

$$
|u|_{\ell}=\sum_{j}\left|\left(\phi_{j}^{-1}\right)^{*} \psi_{j} u\right|_{\ell}, \quad \ell=0,1,
$$

where $\phi_{j}$ stands for Id $\otimes \phi_{j}$.

Norms in all dimensions. For a function $u$ defined on $\left(0, X_{0}\right) \times \mathbb{R}^{n-1} \times \mathbb{R}$ we set

$$
\begin{aligned}
& \|u\|_{0}=\|u\|_{L^{2}\left(\left(0, X_{0}\right) \times \mathbb{R}^{n-1} \times \mathbb{R}\right)}, \\
& \|u\|_{1}^{2}=\left\|D_{x_{0}} u\right\|_{0}^{2}+\int_{0}^{X_{0}} \int_{\mathbb{R}}\|u\|_{\mathscr{H}^{1}\left(\mathbb{R}^{n-1}\right)}^{2} d x_{0} d x_{n}+\left\|D_{x_{n}} u\right\|_{0}^{2} .
\end{aligned}
$$

Note that the latter norm is equivalent to $\|u\|_{\mathscr{H}}\left(\mathbb{R} \times \mathbb{R}^{n-1} \times \mathbb{R}\right)$ if moreover $u$ is compactly supported in the $x_{0}$ variable. For a function $u$ defined on $\left(0, X_{0}\right) \times S \times \mathbb{R}$, we set

$$
\|u\|_{\ell}=\sum_{j}\left\|\left(\phi_{j}^{-1}\right)^{*} \psi_{j} u\right\|_{\ell}, \quad \ell=0,1,
$$

where $\phi_{j}$ stands for Id $\otimes \phi_{j} \otimes$ Id.

The following lemma is a counterpart of Lemma 1.8 when working on a local chart of $\left(0, X_{0}\right) \times S$ or $\left(0, X_{0}\right) \times S \times \mathbb{R}$.

Lemma 1.9. Let $u$ be such that $\operatorname{supp}(u) \subset K \subset\left(0, X_{0}\right) \times U_{j}\left(\right.$ resp. $\left.\left(0, X_{0}\right) \times U_{j} \times \mathbb{R}\right)$ with $K$ compact. Then for some constant $C_{K}$ we have $C_{K}^{-1}|u|_{\ell} \leq\left|\left(\phi_{j}^{-1}\right)^{*} u\right|_{\ell} \leq C_{K}|u|_{\ell}\left(\right.$ resp. $\left.C_{K}^{-1}\|u\|_{\ell} \leq\left\|\left(\phi_{j}^{-1}\right)^{*} u\right\|_{\ell} \leq C_{K}\|u\|_{\ell}\right), \quad \ell=0,1$. 
Proof. We treat the case of a function defined in $\left(0, X_{0}\right) \times U_{j}$. Consider a partition of unity of $S, \sum_{k} \hat{\psi}_{k}=1, \hat{\psi}_{k} \in \mathscr{C}_{c}^{\infty}\left(\left(0, X_{0}\right) \times U_{k}\right)$, such that $1 \otimes \hat{\psi}_{j}=1$ in a neighborhood of $K$. Then the induced norms are equivalent to that given above by Lemma 1.8 and for the particular function $u$ they are equal to $\left|\left(\phi_{j}^{-1}\right)^{*} u\right|_{\ell}, \ell=0,1$.

Tangential semiclassical operators on a manifold. We can define tangential semiclassical operators on a manifold by means of local representations. This relies on the change of variables formula for semiclassical operators in $\mathbb{R}^{d}$ presented in Appendix B.1. We provide details of this construction in Appendix B.2. In particular we define the local symbol of the operator in each chart and its principal symbol on the manifold. We also provide composition and Sobolev regularity results for such operators. In Section 3.6 below we introduce a particular class of tangential operators that will be important in the proof of the Carleman estimate as they will allow us to separate the analysis into microlocal regions.

A trace formula. In the sections below, we shall also use of the following trace formula [LR97, p. 486] connecting the tangential and volume norms introduced above:

$$
\left|\psi_{\mid x_{n}=0^{+}}\right|_{0} \leq C h^{-1 / 2}\|\psi\|_{1}
$$

for $\psi$ defined on $\mathbb{R}^{n+1}$, as well as for $\psi$ defined on $\left(0, X_{0}\right) \times S \times[0,2 \varepsilon]$.

\section{Well-posedness and asymptotic behavior}

We introduce a more general operator

$$
\mathcal{A}_{\delta} Z=\left(\begin{array}{c}
-\Delta_{c} z+\nabla_{a} z+b z \\
-\Delta_{c^{s}} z^{s}+\nabla_{a}^{s} z^{s}+b^{s} z^{s}-\frac{1}{\delta}\left(\left(c \partial_{\eta} z\right)_{\mid S_{2}}-\left(c \partial_{\eta} z\right)_{\mid S_{1}}\right)
\end{array}\right),
$$

with domain $D\left(\mathcal{A}_{\delta}\right)=D\left(A_{\delta}\right)$ (see (1.9)), where $\nabla_{a}$ (resp. $\nabla_{a}^{s}$ ) denotes a smooth vector field $a(x) \nabla_{g}$ (resp. $\left.a^{s}(x) \nabla^{s}\right)$, and $b$ (resp. $b^{s}$ ) is a bounded function.

We start by considering the well-posedness of the evolution problem (1.4), $\partial_{t} Z+$ $\mathcal{A}_{\delta} Z=F$. Note that the lower-order perturbations we add to $A_{\delta}$ to form $\mathcal{A}_{\delta}$ do not affect the well-posedness properties (compare with (1.8)).

\subsection{Well-posedness}

In this section we simply assume that $a, a^{s}$ are bounded coefficients. For $Z, \tilde{Z} \in D\left(A_{\delta}\right)$, an integration by parts gives

$$
\begin{aligned}
\left(\left(\mathcal{A}_{\delta}+\lambda \mathrm{Id}\right) Z, \tilde{Z}\right)_{\mathcal{H}_{\delta}^{0}}= & \left(c \nabla_{g} z, \nabla_{g} \tilde{z}\right)_{L^{2}\left(\Omega_{1} \cup \Omega_{2}\right)}+\left(\nabla_{a} z+(b+\lambda) z, \tilde{z}\right)_{L^{2}\left(\Omega_{1} \cup \Omega_{2}\right)} \\
& +\delta\left(c^{s} \nabla^{s} z^{s}, \nabla^{s} \tilde{z}^{s}\right)_{L^{2}(S)}+\delta\left(\nabla_{a}^{s} z^{s}+\left(b^{s}+\lambda\right) z^{s}, \tilde{z}^{s}\right)_{L^{2}(S)} \\
= & : a_{\lambda}(Z, \tilde{Z}) .
\end{aligned}
$$

The bilinear form $a_{\lambda}$ is in fact continuous on $\left(\mathcal{H}_{\delta}^{1}\right)^{2}$. 
Lemma 2.1. There exists $\lambda_{0} \geq 0$ sufficiently large such that the bilinear form $a_{\lambda}$ is coercive, uniformly in $\delta$, if $\lambda \geq \lambda_{0}$.

Proof. This follows from

$$
\begin{aligned}
a_{\lambda}(Z, Z) \geq & \frac{c_{\min }}{2}\left\|\nabla_{g} z\right\|_{L^{2}\left(\Omega_{1} \cup \Omega_{2}\right)}^{2}+\left(\lambda-\frac{\|a\|_{L^{\infty}\left(\Omega_{1} \cup \Omega_{2}\right)}^{2}}{2 c_{\min }}-\|b\|_{L^{\infty}\left(\Omega_{1} \cup \Omega_{2}\right)}\right)\|z\|_{L^{2}\left(\Omega_{1} \cup \Omega_{2}\right)}^{2} \\
& +\delta \frac{c_{\min }^{s}}{2}\left|\nabla^{s} z^{s}\right|_{L^{2}(S)}^{2}+\delta\left(\lambda-\frac{\left|a^{s}\right|_{L^{\infty}(S)}^{2}}{2 c_{\min }^{s}}-\left|b^{s}\right|_{L^{\infty}(S)}\right)\left|z^{s}\right|_{L^{2}(S)}^{2} .
\end{aligned}
$$

The coercivity of $a_{\lambda}$ shows that the problem $\left(\mathcal{A}_{\delta}+\lambda\right.$ Id $) Z=F$ for $F \in \mathcal{H}_{\delta}^{0}$ is well-posed in a weak sense; for any continuous linear form $L$ on $\mathcal{H}_{\delta}^{1}$, the Lax-Milgram theorem ensures the existence and uniqueness of $Z \in \mathcal{H}_{\delta}^{1}$ satisfying

$$
a_{\lambda}(Z, \tilde{Z})=L(\tilde{Z}) \quad \text { for any } \tilde{Z} \in \mathcal{H}_{\delta}^{1},
$$

and $\|Z\|_{\mathcal{H}_{\delta}^{1}} \leq C\|L\|_{\left(\mathcal{H}_{\delta}^{1}\right)^{\prime}}$ with the constant $C$ uniform in $\delta$. If we take $L(\tilde{Z})=(F, \tilde{Z})_{\mathcal{H}_{\delta}^{0}}$ for some $F \in \mathcal{H}_{\delta}^{0}$, this linear form is continuous on $\mathcal{H}_{\delta}^{1}$. Then, for some constant $C>0$ uniform in $\delta$ the solution satisfies

$$
\|Z\|_{\mathcal{H}_{\delta}^{1}} \leq C\|F\|_{\mathcal{H}_{\delta}^{0}} .
$$

Higher regularity can be obtained.

Proposition 2.2. Let $\lambda \geq \lambda_{0}$ and $F \in \mathcal{H}_{\delta}^{0}$. The unique weak solution $Z=\left(z, z^{s}\right) \in \mathcal{H}_{\delta}^{1}$ to $(2.2)$ with $L(\tilde{Z})=(F, \tilde{Z})_{\mathcal{H}_{\delta}^{0}}$ belongs to $D\left(A_{\delta}\right)$. Hence, for all $F \in \mathcal{H}_{\delta}^{0}$ there exists a unique $Z \in D\left(A_{\delta}\right)$ such that $\mathcal{A}_{\delta} Z+\lambda Z=F$ and moreover for some positive constant $C$ uniform in $\delta$ we have

$$
\sum_{i=1,2}\left\|z_{\mid \Omega_{i}}\right\|_{H^{2}\left(\Omega_{i}\right)}+\delta^{1 / 2}\left|z^{s}\right|_{H^{2}(S)} \leq C\|F\|_{\mathcal{H}_{\delta}^{0}} .
$$

Proposition 2.3. Let $\lambda \geq \lambda_{0}$ and $F=\left(f, f^{s}\right) \in H^{m}\left(\Omega_{1} \cup \Omega_{2}\right) \times H^{m}(S)$. The unique weak solution $Z=\left(z, z^{s}\right) \in \mathcal{H}_{\delta}^{1}$ to $(2.2)$ with $L(\tilde{Z})=(F, \tilde{Z})_{\mathcal{H}_{\delta}^{0}}$ belongs to $H^{m+2}\left(\Omega_{1} \cup \Omega_{2}\right) \times H^{m+2}(S)$ with

$$
\sum_{i=1,2}\left\|\left.z\left|\Omega_{i} \|_{H^{m+2}\left(\Omega_{i}\right)}+\delta^{1 / 2}\right| z^{s}\right|_{H^{m+2}(S)} \leq C\left(\sum_{i=1,2}\left\|f_{\mid \Omega_{i}}\right\|_{H^{m}\left(\Omega_{i}\right)}+\delta^{1 / 2}\left|f^{s}\right|_{H^{m}(S)}\right)\right.
$$

We refer to Appendices C.2 and C. 3 for proofs.

A consequence of the properties we have gathered on $\mathcal{A}_{\delta}$ is the following wellposedness for the evolution problem.

Proposition 2.4. Let $a, b, a^{s}$ and $b^{s}$ be bounded coefficients. Then the operator $\left(-\mathcal{A}_{\delta}, D\left(\mathcal{A}_{\delta}\right)\right)$ generates a $\mathscr{C}^{0}$-semigroup on $\mathcal{H}_{\delta}^{0}$. If moreover $a=0$, $a^{s}=0$ and $b, b^{s} \in \mathbb{R}$, then $\mathcal{A}_{\delta}$ is self-adjoint on $\mathcal{H}_{\delta}^{0}$. 
Proof. Lemma 2.1 shows that $\mathcal{A}_{\delta}+\lambda_{0}$ Id is monotone and Proposition 2.2 shows that this operator maps its domain $D\left(A_{\delta}\right)$ onto $\mathcal{H}_{\delta}^{0}$. Hence $\mathcal{A}_{\delta}+\lambda_{0}$ Id is maximal monotone. The Lumer-Phillips theorem (see e.g. [Paz83]) then allows one to conclude that $\mathcal{A}_{\delta}$ generates a strongly continuous semigroup on $\mathcal{H}_{\delta}^{0}$.

Note that if $a=0, a^{s}=0$ and $b, b^{s} \in \mathbb{R}$, from (2.1) we see that the operator $\mathcal{A}_{\delta}$ is symmetric. It is self-adjoint, as the surjectivity of $\mathcal{A}_{\delta}+\lambda_{0} I$ implies $D\left(\mathcal{A}_{\delta}^{*}\right)=D\left(\mathcal{A}_{\delta}\right)=$ $D\left(A_{\delta}\right)$ (see e.g. [Bre83, Proposition VII-6]).

By the Rellich theorem $\mathcal{H}_{\delta}^{1}$ injects compactly in $\mathcal{H}_{\delta}^{0}$. Hence the inverse $\left(\mathcal{A}_{\delta}+\lambda_{0} \text { Id }\right)^{-1}$ that we constructed is a compact map from $\mathcal{H}_{\delta}^{0}$ into itself. One then deduces the following spectral result.

Proposition 2.5. There exists a Hilbert basis of $\mathcal{H}_{\delta}^{0}$ formed of eigenfunctions, $\mathscr{E}_{j}=$ $\left(e_{\delta, j}, e_{\delta, j}^{s}\right), j \in \mathbb{N}$, of the self-adjoint operator $A_{\delta}$ associated with the eigenvalues $0 \leq$ $\mu_{\delta, 0} \leq \mu_{\delta, 1} \leq \cdots$.

Note that if $\Omega$ is a manifold without boundary then 0 is an eigenfunction for $A_{\delta}$. If $\Omega$ has a boundary, the Dirichlet boundary condition that we prescribe implies the first eigenvalue is positive.

Corollary 2.6. The function space

$$
\mathscr{T}=\left\{\left(z, z^{s}\right) \in \mathcal{H}_{\delta}^{1} ; z_{\mid \Omega_{i}} \in \mathscr{C}^{\infty}\left(\overline{\Omega_{i}}\right), i=1,2\right\}
$$

is dense in $D\left(A_{\delta}\right)$.

Proof. From Proposition 2.3 the eigenfunctions of $A_{\delta}$ are in $\mathscr{T}$. The result follows as they generate a dense subset in $D\left(A_{\delta}\right)$.

\subsection{Asymptotic behavior of solutions as $\delta \rightarrow 0$}

2.2.1. Asymptotic behavior in the elliptic problem. Consider $F_{\delta}=\left(f_{\delta}, f_{\delta}^{s}\right) \in \mathcal{H}_{\delta}^{0}$. Let $Z_{\delta}=\left(z_{\delta}, z_{\delta}^{S}\right)$ be the strong solution defined in the previous section for the elliptic equation $\left(A_{\delta}+\lambda\right) Z_{\delta}=F_{\delta}$.

We also consider the weak solution $z \in H_{0}^{1}$ of the elliptic problem

$$
-\operatorname{div}_{g}\left(c \nabla_{g} z\right)+\lambda z=f \quad \text { in } \Omega .
$$

Arguing as in the previous section such a solution exists and is unique for $\lambda \geq \lambda_{0}$ (the same value of $\lambda_{0}$ as in Lemma 2.1 can be used). In particular we have $z_{\mid S_{1}}=z_{\mid S_{2}}$, i.e. the solution is continuous across the interface, and as $c \nabla_{g} z$ has its divergence in $L^{2}(\Omega)$ we have $c \partial_{\eta} \mathrm{z}_{\mid S_{1}}=c \partial_{\eta} \mathrm{z}_{\mid S_{2}}$. Moreover $\mathrm{z}_{\mid \Omega_{i}} \in H^{2}\left(\Omega_{i}\right)$ and

$$
\sum_{i=1,2}\left\|\mathrm{z} \mid \Omega_{i}\right\|_{H^{2}\left(\Omega_{i}\right)} \leq C\|\mathrm{f}\|_{L^{2}\left(\Omega_{1} \cup \Omega_{2}\right)} .
$$

Proposition 2.7. Suppose that $\left\|F_{\delta}\right\|_{\mathcal{H}_{\delta}^{0}} \leq C$ uniformly in $\delta$ and $f_{\delta} \rightarrow \mathrm{f}$ in $L^{2}\left(\Omega_{1} \cup \Omega_{2}\right)$ as $\delta \rightarrow 0$. Then $z_{\delta \mid \Omega_{j}} \rightarrow \mathrm{z}_{\mid \Omega_{j}}$ in $H^{2}\left(\Omega_{j}\right)$ for $j=1,2$. 
Note that the assumption $\left\|F_{\delta}\right\|_{\mathcal{H}_{\delta}^{0}} \leq C$ implies that there always exists a sequence $\delta_{n} \rightarrow 0$ such that $f_{\delta_{n}} \rightarrow \mathrm{f}$.

Proof. We set $\zeta_{\delta}:=z_{\delta}-z$. According to (2.4), the boundedness assumption on $F_{\delta}$, and (2.7), we have

$$
\sum_{i=1,2}\left\|\zeta_{\delta \mid \Omega_{i}}\right\|_{H^{2}\left(\Omega_{i}\right)} \leq C
$$

uniformly in $\delta$. Moreover, $\zeta_{\delta}$ satisfies

$$
\begin{cases}-\operatorname{div}_{g}\left(c \nabla_{g} \zeta_{\delta}\right)+\lambda \zeta_{\delta}=f_{\delta}-\mathrm{f} & \text { in } \Omega_{1} \cup \Omega_{2}, \\ \left(c \partial_{\eta} \zeta_{\delta}\right)_{\mid S_{2}}-\left(c \partial_{\eta} \zeta_{\delta}\right)_{\mid S_{1}}=\delta\left(-\Delta_{c} z_{\delta}^{s}+\lambda z_{\delta}^{s}-f_{\delta}^{s}\right) & \text { in } S, \\ \zeta_{\delta \mid S_{1}}=\zeta_{\delta \mid S_{2}} & \text { in } S, \\ \zeta_{\delta \mid \partial \Omega}=0 . & \end{cases}
$$

Taking the inner product of the first line of this system with $\zeta_{\delta}$ and integrating by parts, we obtain

$$
\begin{aligned}
\left(c \nabla_{g} \zeta_{\delta}, \nabla_{g} \zeta_{\delta}\right)_{L^{2}\left(\Omega_{1} \cup \Omega_{2}\right)}+\left(\left(c \partial_{\eta} \zeta_{\delta}\right)_{\mid S_{2}}-\left(c \partial_{\eta} \zeta_{\delta}\right)_{\mid S_{1}}, \zeta_{\delta}\right)_{L^{2}(S)}+\lambda\left(\zeta_{\delta}, \zeta_{\delta}\right)_{L^{2}\left(\Omega_{1} \cup \Omega_{2}\right)} & =\left(f_{\delta}-\mathrm{f}, \zeta_{\delta}\right)_{L^{2}\left(\Omega_{1} \cup \Omega_{2}\right)}
\end{aligned}
$$

In this expression, we have

$$
\begin{aligned}
& \left|\left(\left(c \partial_{\eta} \zeta \delta\right)_{\mid S_{2}}-\left(c \partial_{\eta} \zeta \delta\right)_{\mid S_{1}}, \zeta_{\delta}\right)_{L^{2}(S)}\right|=\delta^{1 / 2}\left|\left(\delta^{1 / 2} \Delta_{C^{s}} z_{\delta}^{s}-\delta^{1 / 2} \lambda z_{\delta}^{s}+\delta^{1 / 2} f_{\delta}^{s}, \zeta_{\delta}\right)_{L^{2}(S)}\right| \\
& \leq C \delta^{1 / 2}\left(\delta^{1 / 2}\left|z_{\delta}^{s}\right|_{H^{2}(S)}+\left\|F_{\delta}\right\|_{\mathcal{H}_{\delta}^{0}}\right)\left\|\zeta_{\delta}\right\|_{H^{1}\left(\Omega_{1} \cup \Omega_{2}\right)} \leq C \delta^{1 / 2}\|\zeta \delta\|_{H^{2}\left(\Omega_{1} \cup \Omega_{2}\right)} \rightarrow 0
\end{aligned}
$$

according to (2.4), the trace estimate and the boundedness assumption on $F_{\delta}$. Moreover, since $\zeta_{\delta}$ is bounded in $H^{2}\left(\Omega_{1} \cup \Omega_{2}\right)$, from every sequence $\delta_{n} \rightarrow 0$ we can extract a subsequence, also called $\delta_{n}$, such that $\zeta_{\delta_{n}}$ converges strongly in $L^{2}\left(\Omega_{1} \cup \Omega_{2}\right)$, and we have

As a consequence, we obtain

$$
\left(f_{\delta_{n}}-\mathrm{f}, \zeta_{\delta_{n}}\right)_{L^{2}\left(\Omega_{1} \cup \Omega_{2}\right)} \rightarrow 0 .
$$

$$
\left(c \nabla_{g} \zeta_{\delta_{n}}, \nabla_{g} \zeta_{\delta_{n}}\right)_{L^{2}\left(\Omega_{1} \cup \Omega_{2}\right)}+\lambda\left(\zeta_{\delta_{n}}, \zeta_{\delta_{n}}\right)_{L^{2}\left(\Omega_{1} \cup \Omega_{2}\right)} \rightarrow 0,
$$

i.e. $\zeta_{\delta_{n}} \rightarrow 0$ in $H^{1}\left(\Omega_{j}\right)$, for $j=1$, 2. Because the limit is the same for any subsequence of $\zeta_{\delta_{n}}$, this implies that the whole $\zeta_{\delta}$ converges to zero in $H^{1}\left(\Omega_{j}\right)$. Since $\zeta_{\delta \mid \Omega_{j}}$ is uniformly bounded in $H^{2}\left(\Omega_{j}\right)$, the result follows.

2.2.2. Asymptotic behavior in the parabolic problem. Here, we discuss, for some $\lambda>0$ (one can take $\lambda=0$ if $\partial \Omega \neq \emptyset$ ) the convergence of the solution $Z_{\delta}=\left(z_{\delta}, z_{\delta}^{s}\right)$ of

$$
\begin{cases}\partial_{t} z_{\delta}-\Delta_{c} z_{\delta}+\lambda z_{\delta}=f_{\delta} & \text { in }(0, T) \times \Omega_{1} \cup \Omega_{2}, \\ \partial_{t} z_{\delta}^{s}-\Delta_{c} z_{\delta}^{s}+\lambda z_{\delta}^{s}=\frac{1}{\delta}\left(\left(c \partial_{\eta} z_{\delta}\right)_{\mid S_{2}}-\left(c \partial_{\eta} z_{\delta}\right)_{\mid S_{1}}\right)+f_{\delta}^{s} & \text { in }(0, T) \times S, \\ z_{\delta \mid S_{1}}=z_{\delta}^{s}=z_{\delta \mid S_{2}} & \text { in }(0, T) \times S, \\ z_{\delta \mid \partial \Omega}=0 & \text { in }(0, T), \\ z_{\delta \mid t=0}=z_{0} \quad \text { and } \quad z_{\delta \mid t=0}^{s}=z_{0}^{s}, & \end{cases}
$$


towards the solution $z$ of

$$
\begin{cases}\partial_{t} z-\Delta_{c} z+\lambda z=f & \text { in }(0, T) \times \Omega_{1} \cup \Omega_{2}, \\ z_{\mid S_{1}}=z_{\mid S_{2}} \text { and } \quad\left(c \partial_{\eta} z\right)_{\mid S_{2}}=\left(c \partial_{\eta} z\right)_{\mid S_{1}} & \text { in }(0, T) \times S, \\ z_{\mid \partial \Omega}=0 & \text { in }(0, T), \\ z_{\mid t=0}=z_{0} & \text { in } \Omega .\end{cases}
$$

Proposition 2.8. Suppose that $\left\|F_{\delta}\right\|_{L^{2}\left(0, T ; \mathcal{H}_{\delta}^{0}\right)} \leq C$ uniformly in $\delta, f_{\delta} \rightarrow \mathrm{f}$ in $L^{2}\left((0, T) \times \Omega_{1} \cup \Omega_{2}\right)$ as $\delta \rightarrow 0$ and $z_{0} \in H_{0}^{1}(\Omega)$ and $z_{0}^{s} \in H^{1}(S)$. Then $z_{\delta \mid \Omega_{j}} \rightarrow \mathrm{z}_{\mid \Omega_{j}}$ in $L^{2}\left(0, T ; H^{2}\left(\Omega_{j}\right)\right) \cap H^{1}\left(0, T ; L^{2}\left(\Omega_{j}\right)\right)$ and $*$-weak in $L^{\infty}\left(0, T ; H^{1}\left(\Omega_{j}\right)\right)$, and there exists $C^{\prime}>0$ such that for all $t \in[0, T],\left\|z_{\delta \mid \Omega_{j}}(t)\right\|_{H^{1}\left(\Omega_{j}\right)} \leq C^{\prime}$ for $j=1,2$.

Proof. First, Problem (2.9) can be equivalently rewritten as $\partial_{t} Z_{\delta}+\left(A_{\delta}+\lambda\right) Z_{\delta}=F_{\delta}$ with $Z_{\delta}(0)=\left(z_{0}, z_{0}^{s}\right)$. For $Z_{\delta}(0) \in D\left(A_{\delta}\right)$ and $F_{\delta} \in \mathscr{C}^{0}\left([0, T] ; \mathcal{H}_{\delta}^{0}\right)$ the semigroup solution of this equation is in $\mathscr{C}^{0}\left([0, T] ; D\left(A_{\delta}\right)\right) \cap \mathscr{C}^{1}\left([0, T] ; \mathcal{H}_{\delta}^{0}\right)$ (see [Paz83, Corollary 2.6, Chap. 4] or [Bre83, Théorème VII.10]). As a consequence, we can form the square of the $\mathcal{H}_{\delta}^{0}$ norm of this equation and integrate on $(0, T)$. This yields

$$
\begin{aligned}
& \int_{0}^{T}\left(\frac{d}{d t}\left\|\left(A_{\delta}+\lambda\right)^{1 / 2} Z_{\delta}\right\|_{\mathcal{H}_{\delta}^{0}}^{2}+\left\|\left(A_{\delta}+\lambda\right) Z_{\delta}(t)\right\|_{\mathcal{H}_{\delta}^{0}}^{2}+\left\|\partial_{t} Z_{\delta}(t)\right\|_{\mathcal{H}_{\delta}^{0}}^{2}\right) d t \\
&=\int_{0}^{T}\left\|F_{\delta}(t)\right\|_{\mathcal{H}_{\delta}^{0}}^{2} d t
\end{aligned}
$$

which in turns gives the stability estimate for the solution of (2.9):

$$
\begin{aligned}
&\left\|Z_{\delta}(T)\right\|_{\mathcal{H}_{\delta}^{1}}^{2}+\int_{0}^{T}\left\|\left(A_{\delta}+\lambda\right) Z_{\delta}(t)\right\|_{\mathcal{H}_{\delta}^{0}}^{2} d t+\int_{0}^{T}\left\|\partial_{t} Z_{\delta}(t)\right\|_{\mathcal{H}_{\delta}^{0}}^{2} d t \\
& \leq C\left(\int_{0}^{T}\left\|F_{\delta}(t)\right\|_{\mathcal{H}_{\delta}^{0}}^{2} d t+\left\|Z_{\delta}(0)\right\|_{\mathcal{H}_{\delta}^{1}}^{2}\right),
\end{aligned}
$$

uniformly in $\delta$. By a density argument, this energy estimate remains valid if $Z_{\delta}(0) \in \mathcal{H}_{\delta}^{1}$ and $F_{\delta}(t) \in L^{2}\left(0, T ; \mathcal{H}_{\delta}^{0}\right)$.

According to (2.4), this yields

$$
\begin{aligned}
\left\|z_{\delta}(T)\right\|_{H^{1}}^{2}+\delta\left|z_{\delta}^{S}(T)\right|_{H^{1}(S)}^{2}+\int_{0}^{T}\left\|z_{\delta}(t)\right\|_{H^{2}}^{2} d t+\delta \int_{0}^{T}\left|z_{\delta}^{S}(t)\right|_{H^{2}(S)}^{2} d t+\int_{0}^{T}\left\|\partial_{t} z_{\delta}(t)\right\|_{L^{2}}^{2} d t \\
\quad+\delta \int_{0}^{T}\left|\partial_{t} z_{\delta}^{S}(t)\right|_{L^{2}(S)}^{2} d t \leq C\left(\int_{0}^{T}\left\|F_{\delta}(t)\right\|_{\mathcal{H}_{\delta}^{0}}^{2} d t+\left\|Z_{\delta}(0)\right\|_{\mathcal{H}_{\delta}^{1}}^{2}\right) \leq C, \quad
\end{aligned}
$$

uniformly in $\delta$ (the volume norms are taken over $\Omega_{1} \cup \Omega_{2}$ ).

In addition, the solution of (2.10) also satisfies

$$
\|\mathrm{z}(T)\|_{H^{1}}^{2}+\int_{0}^{T}\left(\left\|\left(-\Delta_{c}+\lambda\right) \mathrm{z}(t)\right\|_{L^{2}}^{2}+\left\|\partial_{t} \mathrm{z}(t)\right\|_{L^{2}}^{2}\right) d t \leq C\left(\int_{0}^{T}\|\mathrm{f}(t)\|_{L^{2}}^{2} d t+\|\mathrm{z}(0)\|_{H^{1}}^{2}\right),
$$


where all the norms are taken over $\Omega_{1} \cup \Omega_{2}$. By using the additional regularity (2.7), this gives

$$
\|\mathrm{z}(T)\|_{H^{1}}^{2}+\int_{0}^{T}\left(\|\mathrm{z}(t)\|_{H^{2}}^{2}+\left\|\partial_{t} \mathrm{z}(t)\right\|_{L^{2}}^{2}\right) d t \leq C\left(\int_{0}^{T}\|\mathrm{f}(t)\|_{L^{2}}^{2} d t+\|\mathrm{z}(0)\|_{H^{1}}^{2}\right) .
$$

Now, we set $\zeta_{\delta}=z_{\delta}-z$. According to (2.11)-(2.12), we have

$$
\sum_{j=1,2}\left(\left\|\zeta_{\delta \mid \Omega_{j}}\right\|_{L^{\infty}\left(0, T ; H^{1}\left(\Omega_{j}\right)\right)}+\left\|\zeta_{\delta \mid \Omega_{j}}\right\|_{L^{2}\left(0, T ; H^{2}\left(\Omega_{j}\right)\right)}+\left\|\zeta_{\delta \mid \Omega_{j}}\right\|_{H^{1}\left(0, T ; L^{2}\left(\Omega_{j}\right)\right)}\right) \leq C,
$$

uniformly in $\delta$. Moreover, $\zeta_{\delta}$ satisfies

$$
\begin{cases}\partial_{t} \zeta_{\delta}-\Delta_{c} \zeta_{\delta}+\lambda \zeta_{\delta}=f_{\delta}-\mathrm{f} & \text { in }(0, T) \times\left(\Omega_{1} \cup \Omega_{2}\right) \\ \left(c \partial_{\eta} \zeta_{\delta}\right)_{\mid S_{2}}-\left(c \partial_{\eta} \zeta_{\delta}\right)_{\mid S_{1}}=\delta\left(\partial_{t} z_{\delta}^{s}-\Delta_{c} z_{\delta}^{s}+\lambda z_{\delta}^{s}-f_{\delta}^{s}\right) & \text { in }(0, T) \times S \\ \zeta_{\delta \mid S_{1}}=\zeta_{\delta \mid S_{2}} & \text { in }(0, T) \times S \\ \zeta_{\delta \mid \partial \Omega}=0 & \text { in }(0, T) \\ \zeta_{\delta \mid t=0}=0 & \text { in } \Omega .\end{cases}
$$

Forming the inner product of the first line of this system with $\zeta_{\delta}$ and integrating on $(0, T)$, we obtain

$$
\begin{aligned}
\frac{1}{2}\left\|\zeta_{\delta}(T)\right\|_{L^{2}(\Omega)}^{2} & +\left\|\sqrt{c} \nabla_{g} \zeta_{\delta}\right\|_{L^{2}((0, T) \times \Omega)}^{2}+\lambda\left\|\zeta_{\delta}\right\|_{L^{2}((0, T) \times \Omega)}^{2} \\
& +\left(\left(c \partial_{\eta} \zeta_{\delta}\right)_{\mid S_{2}}-\left(c \partial_{\eta} \zeta_{\delta}\right)_{\mid S_{1}}, \zeta_{\delta}\right)_{L^{2}((0, T) \times S)}=\left(f_{\delta}-\mathrm{f}, \zeta_{\delta}\right)_{L^{2}((0, T) \times \Omega)}
\end{aligned}
$$

In this expression, we have

$$
\begin{aligned}
\mid\left(\left(c \partial_{\eta} \zeta_{\delta}\right)_{\mid S_{2}}-\right. & \left.\left(c \partial_{\eta} \zeta \delta\right)_{\mid S_{1}}, \zeta \delta\right)_{L^{2}((0, T) \times S)} \mid \\
& =\delta^{1 / 2}\left|\left(\delta^{1 / 2} \partial_{t} z_{\delta}^{s}-\delta^{1 / 2} \Delta_{c^{s}} z_{\delta}^{s}+\delta^{1 / 2} \lambda z_{\delta}^{s}-\delta^{1 / 2} f_{\delta}^{s}, \zeta \delta\right)_{L^{2}((0, T) \times S)}\right| \\
& \leq C \delta^{1 / 2}\left\|\zeta_{\delta}\right\|_{L^{2}\left(0, T ; H^{2}\left(\Omega_{1} \cup \Omega_{2}\right)\right)} \rightarrow 0,
\end{aligned}
$$

according to (2.11) (proceeding as in (2.8)). As in the proof of Proposition 2.7, for a subsequence we have

$$
\left(f_{\delta}-\mathrm{f}, \zeta_{\delta}\right)_{L^{2}\left((0, T) \times\left(\Omega_{1} \cup \Omega_{2}\right)\right)} \rightarrow 0,
$$

and we obtain

$$
\frac{1}{2}\left\|\zeta_{\delta}(T)\right\|_{L^{2}(\Omega)}^{2}+\left\|\sqrt{c} \nabla_{g} \zeta_{\delta}\right\|_{L^{2}((0, T) \times \Omega)}^{2}+\lambda\left\|\zeta_{\delta}\right\|_{L^{2}((0, T) \times \Omega)}^{2} \rightarrow 0 .
$$

This, together with (2.13), concludes the proof of the proposition.

As a consequence, we can obtain a convergence result for the control problem under study. We denote by $u_{\delta}$ the control function given by Theorem 1.6, which satisfies

$$
\left\{\begin{array}{l}
\partial_{t} Z_{\delta}+A_{\delta} Z_{\delta}=B u_{\delta}, \\
Z_{\delta \mid t=0}=Z_{0}, \\
Z_{\delta \mid t=T}=0
\end{array}\right.
$$


According to Theorem 1.6, $u_{\delta}$ is uniformly bounded in $L^{2}((0, T) \times \omega)$, so that we can extract a subsequence (also denoted by $u_{\delta}$ ) weakly converging in this space towards $u$. We also consider the solution $\tilde{Z}_{\delta}=\left(\tilde{z}_{\delta}, \tilde{z}_{\delta}^{s}\right)$ of

$$
\left\{\begin{array}{l}
\partial_{t} \tilde{Z}_{\delta}+A_{\delta} \tilde{Z}_{\delta}=B \mathrm{u} \\
\tilde{Z}_{\delta \mid t=0}=Z_{0}
\end{array}\right.
$$

The following result is a consequence of Proposition 2.8.

Corollary 2.9. The limit $\mathrm{u}$ is a null-control function for the limit system (1.10). Moreover, $\left(\tilde{z}_{\delta}-z_{\delta}\right)_{\mid \Omega_{j}} \rightarrow 0$ in $L^{2}\left(0, T ; H^{2}\left(\Omega_{j}\right)\right) \cap H^{1}\left(0, T ; L^{2}\left(\Omega_{j}\right)\right)$ and *-weak in $L^{\infty}\left(0, T ; H^{1}\left(\Omega_{j}\right)\right)$, and there exists $C>0$ such that for all $t \in[0, T]$, $\left\|z_{\delta \mid \Omega_{j}}(t)-\tilde{z}_{\delta \mid \Omega_{j}}(t)\right\|_{H^{1}\left(\Omega_{j}\right)} \leq C$ for $j=1,2$.

In particular, $\tilde{z}_{\delta}(T) \rightarrow 0$ in $H^{1}(\Omega)$. This shows that the limit $\mathrm{u}$ is a control function for the limit system (1.10) which is robust with respect to small viscous perturbations. Indeed, it realizes an approximate control for System (2.15).

\section{Local setting in a neighborhood of the interface}

In a sufficiently small neighborhood of $S$, say $V_{\varepsilon}$, we can use normal geodesic coordinates (with respect to the spatial variables $x$ ). More precisely (see [Hör85a, Appendix C.5]) for $\varepsilon$ sufficiently small, there exists a diffeomorphism

$$
\mathrm{F}: S \times[-2 \varepsilon, 2 \varepsilon] \rightarrow V_{\varepsilon}, \quad\left(y, x_{n}\right) \mapsto \mathrm{F}\left(y, x_{n}\right),
$$

so that the differential operator $-\partial_{x_{0}}^{2}-\Delta_{c}+\nabla_{a}$ takes the form, on both sides of the interface,

$$
-\partial_{x_{0}}^{2}-c\left(y, x_{n}\right)\left(\partial_{x_{n}}^{2}-R_{2}\left(y, x_{n}\right)\right)+R_{1}\left(y, x_{n}\right),
$$

and the differential operator $-\partial_{x_{0}}^{2}-\Delta_{c}^{s}+\nabla_{a}^{s}$ on the interface takes the form

$$
-\partial_{x_{0}}^{2}+c^{s}(y) R_{2}\left(y, x_{n}=0\right)+R_{1}^{s}(y),
$$

where $R_{2}\left(y, x_{n}\right)$ is an $x_{n}$-family of second-order elliptic differential operators on $S$, i.e., a tangential operator, with principal symbol $r\left(y, x_{n}, \eta\right), \eta \in T_{y}^{*}(S)$, that satisfies

$$
r\left(y, x_{n}, \eta\right) \in \mathbb{R} \quad \text { and } \quad C_{1}|\eta|_{g}^{2} \leq r\left(y, x_{n}, \eta\right) \leq C_{2}|\eta|_{g}^{2},
$$

for some $0<C_{1} \leq C_{2}<\infty$, and $R_{1}\left(y, x_{n}\right)$ is a first-order operator on $S \times([-2 \varepsilon, 0) \cup$ $(0,2 \varepsilon])$ (involving partial derivatives in all variables and having a jump across $S \times\{0\}$ ); finally $R_{1}^{S}(y)$ is a first-order operator on $S$.

By abuse of notation we shall write $V_{\varepsilon}$ in place of $S \times[-2 \varepsilon, 2 \varepsilon]$. In this setting,

$$
V_{\varepsilon}^{-}=\mathrm{F}(S \times[-2 \varepsilon, 0))=V_{\varepsilon} \cap \Omega_{1}, \quad V_{\varepsilon}^{+}=\mathrm{F}(S \times(0,2 \varepsilon])=V_{\varepsilon} \cap \Omega_{2},
$$

and we recall that the observation region $\omega$ is in $\Omega_{2}$. 
We shall often write

$$
x:=\left(y, x_{n}\right), \quad \mathrm{x}:=\left(x_{0}, x\right)=\left(x_{0}, y, x_{n}\right) \in\left[0, X_{0}\right] \times S \times[-2 \varepsilon, 2 \varepsilon] .
$$

We set

$P=-\frac{1}{c} \partial_{x_{0}}^{2}-\left(\partial_{x_{n}}^{2}-R_{2}(x)\right)+\frac{1}{c} R_{1}(x), \quad P^{s}=-\frac{1}{c^{s}} \partial_{x_{0}}^{2}+R_{2}\left(y, x_{n}=0\right)+\frac{1}{c^{s}} R_{1}^{s}(y)$.

They both have smooth coefficients.

In this framework, in the neighborhood $V_{\varepsilon}$ of $S$, System (1.11) becomes

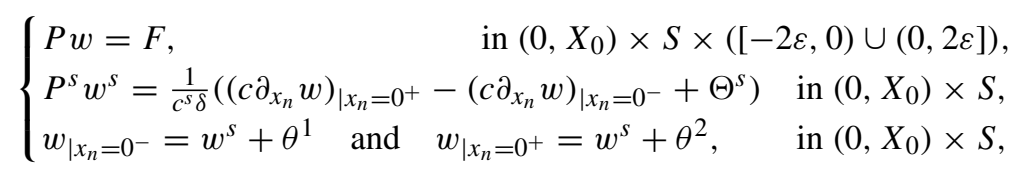

with

$$
F=\frac{1}{c} f+R_{0} w, \quad \Theta^{s}=\theta^{s}+\delta R_{0}^{s} w^{s},
$$

where $R_{0}$ and $R_{0}^{S}$ are zero-order operators with bounded coefficients on $S \times([-2 \varepsilon, 0) \cup$ $(0,2 \varepsilon])$ and $S$ respectively.

\subsection{Properties of weight functions}

We denote by $\tilde{r}\left(x, \eta, \eta^{\prime}\right)$ the symmetric bilinear form associated with the quadratic principal symbol $r(x, \eta)$. We introduce the symmetric bilinear form

$$
\tilde{\beta}\left(x ; \xi_{0}, \eta ; \xi_{0}^{\prime}, \eta^{\prime}\right)=\frac{1}{c(x)} \xi_{0} \xi_{0}^{\prime}+\tilde{r}\left(x, \eta, \eta^{\prime}\right)
$$

and the associated positive definite quadratic form $\beta\left(x ; \xi_{0}, \eta\right)$. We choose a positive bounded continuous function $\gamma(x)$ in $\overline{V_{\varepsilon}^{+}}$such that

$\beta\left(y,-x_{n} ; \xi_{0}, \eta\right)-\gamma\left(y, x_{n}\right) \beta\left(y, x_{n} ; \xi_{0}, \eta\right) \geq C\left|\left(\xi_{0}, \eta\right)\right|^{2}>0,\left(\xi_{0}, \eta\right) \in \mathbb{R} \times T_{y}^{*}(S)$,

for $x=\left(y, x_{n}\right) \in \overline{V_{\varepsilon}^{+}}$.

We then choose a function $\varphi=\varphi(x)$ on $\left[0, X_{0}\right] \times V_{\varepsilon}$ that is smooth on both sides of the interface and simply continuous across the interface, and moreover has the following properties:

1. For a function $\gamma^{\prime}$ such that $0<\gamma^{\prime}(x) \leq \gamma(x)-\epsilon$ in $\overline{V_{\varepsilon}^{+}}$, for some $\epsilon>0$, we have

$$
\gamma^{\prime}\left(y, x_{n}\right)\left(\partial_{x_{n}} \varphi\right)^{2}\left(x_{0}, y, x_{n}\right)-\left(\partial_{x_{n}} \varphi\right)^{2}\left(x_{0}, y,-x_{n}\right) \geq C>0,
$$

for $x_{0} \in\left[0, X_{0}\right]$, and $x=\left(y, x_{n}\right) \in V_{\varepsilon}^{+}$.

2. For a given value of $v>0$ sufficiently small we have

$$
\left|\partial_{x_{0}} \varphi(\mathrm{x})\right|+\left|\nabla^{s} \varphi(\mathrm{x})\right|_{g} \leq \operatorname{vinf}_{\overline{V_{\varepsilon}}}\left|\partial_{x_{n}} \varphi\right|, \quad \mathrm{x}=\left(x_{0}, x\right) \in\left[0, X_{0}\right] \times V_{\varepsilon} .
$$

3. We have $\left|\partial_{x_{0}} \varphi\right|+\left|\nabla^{s} \varphi\right|_{g}+\left|\partial_{x_{n}} \varphi\right|>0$ in $\left[0, X_{0}\right] \times V_{\varepsilon}$ and Hörmander's subellipticity condition is satisfied on both sides of the interface. This condition will be precisely stated below after the introduction of the conjugate operator (see (3.18)). 
Note that $\inf _{V_{\varepsilon}^{+}}\left|\partial_{x_{n}} \varphi\right| \geq C>0$.

The first condition states the increase in the normal slope of the weight function when crossing the interface. The value of $v$ in the second condition will be determined below (see (3.19)-(3.20) and the proof of Proposition 3.5). We thus require the weight function to be relatively flat in the tangent directions to the interface as compared to its variations in the normal direction. We explain below how a weight function satisfying the subellipticity condition can be built through a convexification procedure (see Remark 3.3).

Remark 3.1. Property (3.6) and $\left|\partial_{x_{0}} \varphi\right|+\left|\nabla^{s} \varphi\right|_{g}+\left|\partial_{x_{n}} \varphi\right|>0$ can be obtained by choosing $\varphi$ such that $\left(\partial_{x_{n}} \varphi\right)_{\mid\left[0, X_{0}\right] \times S} \geq C>0$ and assuming that (3.6) only holds on $\left[0, X_{0}\right] \times S$ and then shrinking the neighborhood $V_{\varepsilon}$ by choosing $\varepsilon$ sufficiently small.

An example of such a function will be given in the application of the Carleman estimate in Section 5.

Remark 3.2. Note that the conditions we impose on the weight function are much simpler than the conditions given in [LR10]. Such conditions are proven to be sharp in [LL11] in the limiting case $\delta \rightarrow 0$. If (3.6) is not satisfied, i.e., the increase in the normal slope of the weight function is chosen too small, one can build a quasi-mode that concentrates at the interface and shows that the Carleman estimate cannot hold.

\subsection{A system formulation}

Following [Be103, LR10], we shall consider (3.2) as a system of two equations coupled at the boundary $x_{n}=0^{+}$. Here, the coupling involves a tangential second-order elliptic operator. In $\left[0, X_{0}\right] \times S \times[-2 \varepsilon, 0)$, we make the change of variables $x_{n}$ to $-x_{n}$. For a function $\psi$ defined in $V_{\varepsilon}$, we set

$$
\psi^{r}\left(y, x_{n}\right)=\psi\left(y, x_{n}\right) \quad \text { and } \quad \psi^{l}\left(y, x_{n}\right):=\psi\left(y,-x_{n}\right), \quad \text { for } x_{n} \geq 0,
$$

and similarly for symbols and operators, e.g.,

$$
r^{r}\left(y, x_{n}, \eta\right)=r\left(y, x_{n}, \eta\right) \quad \text { and } \quad r^{l}\left(y, x_{n}, \eta\right)=r\left(y,-x_{n}, \eta\right), \quad \text { for } x_{n} \geq 0 .
$$

We set $V_{\varepsilon}^{+}=S \times(0,2 \varepsilon]$. System (3.2) then takes the form

$$
\begin{cases}P^{r / l} w^{r / l}=F^{r / l}, & \text { in }\left(0, X_{0}\right) \times V_{\varepsilon}^{+}, \\ P^{s} w^{s}=\frac{1}{c^{s} \delta}\left(\left(c^{r} \partial_{x_{n}} w^{r}\right)_{\mid x_{n}=0^{+}}+\left(c^{l} \partial_{x_{n}} w^{l}\right)_{\mid x_{n}=0^{+}}+\Theta^{s}\right) & \text { in }\left(0, X_{0}\right) \times S, \\ w_{\mid x_{n}=0^{+}}^{r / l}=w^{s}+\theta^{r / l} & \text { in }\left(0, X_{0}\right) \times S .\end{cases}
$$

\subsection{Conjugation by a weight function}

We now consider the weight functions $\varphi^{r / l}$ built up as above from the continuous function $\varphi$ defined on $V_{\varepsilon}$. We introduce the following conjugate differential operators:

$$
P_{\varphi}^{r / l}=h^{2} e^{\varphi^{r / l} / h} P^{r / l} e^{-\varphi^{r / l} / h}, \quad P_{\varphi}^{S}=h^{2} e^{\varphi_{\mid S} / h} P^{s} e^{-\varphi_{\mid S} / h} .
$$


With the functions

$$
\begin{aligned}
& v^{r / l}=e^{\varphi^{r / l} / h} w^{r / l}, \quad v^{s}=e^{\varphi_{\mid S} / h} w^{s}, \\
& F_{\varphi}^{r / l}=h^{2} e^{\varphi^{r / l} / h} F^{r / l}, \quad \Theta_{\varphi}^{s}=-i h e^{\varphi \mid S / h} \Theta^{s}, \quad \theta_{\varphi}^{r / l}=e^{\varphi \mid S / h} \theta^{r / l},
\end{aligned}
$$

for $0<h<h_{0}$, System (3.8) can be rewritten as

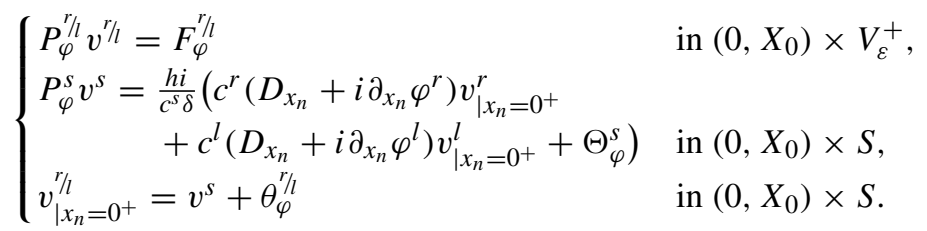

Recall that $D=h \partial / i$ here. We shall consider the operators $P_{\varphi}^{r / l}$ and $P_{\varphi}^{s}$ as semiclassical differential operators.

We separate the self-adjoint and anti-self-adjoint parts of the operators $P_{\varphi}^{r / l}$, viz.,

$$
\tilde{Q}_{2}^{r / l}=\frac{1}{2}\left(P_{\varphi}^{r / l}+\left(P_{\varphi}^{r / l}\right)^{*}\right) . \quad \tilde{Q}_{1}^{r / l}=\frac{1}{2 i}\left(P_{\varphi}^{r / l}-\left(P_{\varphi}^{r / l}\right)^{*}\right)
$$

The (semiclassical) principal symbols $\tilde{q}_{j}$ of $\tilde{Q}_{j}, j=1,2$, are then

$$
\begin{aligned}
& \tilde{q}_{2}^{r / l}\left(\mathrm{x}, \xi_{0}, \eta, \xi_{n}\right)=\xi_{n}^{2}+q_{2}^{r / l}\left(\mathrm{x}, \xi_{0}, \eta\right), \\
& \tilde{q}_{1}^{r / l}\left(\mathrm{x}, \xi_{0}, \eta, \xi_{n}\right)=2 \xi_{n} \partial_{x_{n}} \varphi^{r / l}+2 q_{1}^{r / l}\left(\mathrm{x}, \xi_{0}, \eta\right),
\end{aligned}
$$

for $(y, \eta) \in T^{*}(S)$, with

$$
\begin{aligned}
& q_{2}^{r / l}\left(\mathrm{x}, \xi_{0}, \eta\right)=\frac{\xi_{0}^{2}}{c^{r / l}}+r^{r / l}(x, \eta)-\left(\frac{\left(\partial_{x_{0}} \varphi^{r / l}\right)^{2}}{c^{r / l}}+r^{r / l}\left(x, d_{y} \varphi^{r / l}\right)+\left(\partial_{x_{n}} \varphi^{r / l}\right)^{2}\right), \\
& q_{1}^{r / l}\left(\mathrm{x}, \xi_{0}, \eta\right)=\frac{\xi_{0} \partial_{x_{0}} \varphi^{r / l}}{c^{r / l}}+\tilde{r}^{r / l}\left(x ; \eta, d_{y} \varphi^{r / l}\right) .
\end{aligned}
$$

Recall that $\tilde{r}^{r / l}\left(x, \eta, \eta^{\prime}\right)$ stands for the symmetric bilinear form associated with the quadratic principal symbol $r^{r / l}(x, \eta)$. The principal symbol of $P_{\varphi}^{r / l}$ is naturally

$$
p_{\varphi}^{r / l}=\tilde{q}_{2}^{r / l}+i \tilde{q}_{1}^{r / l}=\xi_{n}^{2}+2 i \xi_{n} \partial_{x_{n}} \varphi^{r / l}+q_{2}^{r / l}+2 i q_{1}^{r / l} .
$$

For concision we sometimes omit variable dependencies, e.g. writing $\varphi^{r / l}$ in place of $\varphi^{r / l}(\mathrm{x})$.

Note also that the symbol of $P_{\varphi}^{s}$ is given by

$$
\begin{aligned}
p_{\varphi}^{s}= & \frac{\xi_{0}^{2}}{c^{s}}+r(x, \eta)-\left.\left(\frac{\left(\partial_{x_{0}} \varphi\right)^{2}}{c^{s}}+r\left(x, d_{y} \varphi_{\mid x_{n}=0}\right)\right)\right|_{x_{n}=0} \\
& +\left.2 i\left(\frac{\xi_{0} \partial_{x_{0}} \varphi}{c^{s}}+\tilde{r}\left(x ; \eta, d_{y} \varphi_{\mid x_{n}=0}\right)\right)\right|_{x_{n}=0} .
\end{aligned}
$$

(Recall that $r^{l}$ and $r^{r}$ (resp. $\varphi^{l}$ and $\varphi^{r}$ ) coincide at $x_{n}=0^{+}$.) 


\subsection{Phase-space regions}

Following [LR97, LR10] we introduce the quantity

$$
\mu^{r / l}\left(\mathrm{x}, \xi_{0}, \eta\right)=q_{2}^{r / l}\left(\mathrm{x}, \xi_{0}, \eta\right)+\frac{\left(q_{1}^{r / l}\left(\mathrm{x}, \xi_{0}, \eta\right)\right)^{2}}{\left(\partial_{x_{n}} \varphi^{r / l}\right)^{2}},
$$

and the following sets in the (tangential) phase space:

$E^{r / l, \pm}=\left\{\left(x_{0}, y, x_{n} ; \xi_{0}, \eta\right) \in\left[0, X_{0}\right] \times S \times[0,2 \varepsilon] \times \mathbb{R} \times T_{y}^{*}(S) ;\right.$

$$
\left.\mu^{r / l}\left(x_{0}, y, x_{n} ; \xi_{0}, \eta\right) \gtrless 0\right\},
$$

$Z^{r / l}=\left\{\left(x_{0}, y, x_{n} ; \xi_{0}, \eta\right) \in\left[0, X_{0}\right] \times S \times[0,2 \varepsilon] \times \mathbb{R} \times T_{y}^{*}(S) ;\right.$

$$
\left.\mu^{r / l}\left(x_{0}, y, x_{n} ; \xi_{0}, \eta\right)=0\right\} \text {. }
$$

The analysis we carry out will specify the behavior of the roots of $p_{\varphi}^{r / l}$ (viewing $p_{\varphi}^{r / l}$ as a second-order polynomial in the variable $\xi_{n}$, see (3.10)) as $\left(x, \xi_{0}, \eta\right)$ varies. In particular, we prove that $\left(\mathrm{x}, \xi_{0}, \eta\right) \in Z^{r / l}$, i.e. $\mu^{r / l}\left(\mathrm{x}, \xi_{0}, \eta\right)=0$, if and only if there exists $\xi_{n} \in \mathbb{R}$ such that $\left(\mathrm{x}, \xi_{0}, \eta, \xi_{n}\right) \in \operatorname{Char}\left(P_{\varphi}^{r / l}\right)$.

With the symmetric bilinear forms

$$
\begin{aligned}
\tilde{\beta}^{r / l}\left(x ; \xi_{0}, \eta ; \xi_{0}^{\prime}, \eta^{\prime}\right) & =\frac{1}{c^{r / l}} \xi_{0} \xi_{0}^{\prime}+\tilde{r}^{r / l}\left(x, \eta, \eta^{\prime}\right), \\
\tilde{\alpha}^{r / l}\left(x ; \xi_{0}, \eta, \xi_{n} ; \xi_{0}^{\prime}, \eta^{\prime}, \xi_{n}^{\prime}\right) & =\tilde{\beta}^{r / l}\left(x ; \xi_{0}, \eta ; \xi_{0}^{\prime}, \eta^{\prime}\right)+\xi_{n} \xi_{n}^{\prime},
\end{aligned}
$$

and the associated quadratic forms, $\beta^{r / l}\left(x ; \xi_{0}, \eta\right)$ and $\alpha^{r / l}\left(x ; \xi_{0}, \eta, \xi_{n}\right)$, we have

$$
\begin{aligned}
\mu^{r / l}\left(\mathrm{x}, \xi_{0}, \eta\right)= & \beta^{r / l}\left(x, \xi_{0}, \eta\right)+\frac{\left(\tilde{\beta}^{r / l}\left(x ; \xi_{0}, \eta ; \partial_{x_{0}} \varphi^{r / l}, d_{y} \varphi^{r / l}\right)\right)^{2}}{\left(\partial_{x_{n}} \varphi^{r / l}\right)^{2}} \\
& -\alpha^{r / l}\left(x ; \partial_{x_{0}} \varphi^{r / l}, d_{y} \varphi^{r / l}, \partial_{x_{n}} \varphi^{r / l}\right) .
\end{aligned}
$$

We also define the quadratic form

$$
\beta_{\varphi}^{r / l}\left(\mathrm{x} ; \xi_{0}, \eta\right)=\left(\tilde{\beta}^{r / l}\left(x ; \xi_{0}, \eta ; \partial_{x_{0}} \varphi^{r / l}, d_{y} \varphi^{r / l}\right)\right)^{2} .
$$

The quadratic forms $\beta^{r / l}$ are positive definite. With the function $\gamma(x)$ on $\overline{V_{\varepsilon}^{+}}$chosen in Section 3.1 we have

$$
\beta^{l}\left(x ; \xi_{0}, \eta\right)-\gamma(x) \beta^{r}\left(x ; \xi_{0}, \eta\right) \geq C\left|\left(\xi_{0}, \eta\right)\right|^{2}>0 .
$$

From the properties of the weight function listed in Section 3.1 we have

$$
\gamma^{\prime}\left(\partial_{x_{n}} \varphi^{r}\right)^{2}-\left(\partial_{x_{n}} \varphi^{l}\right)^{2} \geq C>0, \quad 0<\gamma^{\prime}(x) \leq \gamma(x)-\epsilon, \epsilon>0,
$$

and

$$
\left|\partial_{x_{0}} \varphi^{r / l}\right|+\left|d_{y} \varphi^{r / l}\right|_{g} \leq v \inf \left(\left|\partial_{x_{n}} \varphi^{l}\right|\right)
$$


with $v>0$ sufficiently small. Furthermore $\left|\partial_{x_{0}} \varphi^{r / h}\right|+\left|\nabla_{g} \varphi^{r / h}\right|+\left|\partial_{x_{n}} \varphi^{r / h}\right|>0$ in $\left[0, X_{0}\right] \times \overline{V_{\varepsilon}^{+}}$, and the following subellipticity property is satisfied:

$$
\begin{aligned}
\forall x \in\left[0, X_{0}\right] \times \overline{V_{\varepsilon}^{+}},\left(\xi_{0}, \eta, \xi_{n}\right) \in \mathbb{R} \times T_{y}^{*}(S) \times \mathbb{R}, \\
p_{\varphi}^{r / l}\left(\mathrm{x}, \xi_{0}, \eta, \xi_{n}\right)=0 \Rightarrow\left\{\tilde{q}_{2}^{r / h}, \tilde{q}_{1}^{r / l}\right\}\left(\mathrm{x}, \xi_{0}, \eta, \xi_{n}\right)>0 .
\end{aligned}
$$

The subellipticity property (3.18) is necessary for the derivation of the Carleman estimate and is geometrically invariant (see e.g. [Hör63, Section 8.1, p. 186]; see also [LL12]).

Remark 3.3. A weight function $\varphi$ with the properties of Section 3.1, or (3.16)-(3.18) equivalently, can be obtained in the following classical way. Choose a continuous function $\psi$, smooth on both sides of $S$, such that $\psi^{r / h}$ satisfies conditions (3.16), (3.17) and $\left|\partial_{x_{0}} \psi^{r / l}\right|+\left|\nabla^{s} \psi^{r / l}\right|_{g}+\left|\partial_{x_{n}} \psi^{r / l}\right|>0$ on $\left[0, X_{0}\right] \times \overline{V_{\varepsilon}^{+}}$. These conditions are then also satisfied by $\varphi=e^{\lambda \psi}, \lambda \geq 1$. For the parameter $\lambda$ sufficiently large, $\varphi$ will also fulfill the subellipticity condition (see e.g. Lemma 3 in [LR95, Section 3.B], Theorem 8.6.3 in [Hör63, Chapter 8], or Proposition 28.3.3 in [Hör85b, Chapter 28]).

Using (3.15)-(3.17) for $v$ sufficiently small, we obtain

$$
\beta^{l}-\gamma(x)\left(\beta^{r}+\beta_{\varphi}^{r} /\left(\partial_{x_{n}} \varphi^{r}\right)^{2}\right) \geq C\left|\left(\xi_{0}, \eta\right)\right|^{2}>0,
$$

and

$$
\gamma(x) \alpha^{r}\left(x ; \partial_{x_{0}} \varphi^{r}, d_{y} \varphi^{r}, \partial_{x_{n}} \varphi^{r}\right)-\alpha^{l}\left(x ; \partial_{x_{0}} \varphi^{l}, d_{y} \varphi^{l}, \partial_{x_{n}} \varphi^{l}\right) \geq C>0,
$$

where we have used that $\gamma \geq \gamma^{\prime}+\varepsilon$.

The assumption we have formulated yields the following key property.

Proposition 3.4. There exists $C_{0}>0$ such that in the neighborhood $V_{\varepsilon}$ we have

$$
\begin{aligned}
& \left(\mu^{l}-\gamma(x) \mu^{r}\right)\left(\mathrm{x}, \xi_{0}, \eta\right) \geq C_{0}\left\langle\left(\xi_{0}, \eta\right)\right\rangle^{2}>0, \\
& \quad \mathrm{x}=\left(x_{0}, x\right)=\left(x_{0}, y, x_{n}\right),\left(\xi_{0}, \eta\right) \in \mathbb{R} \times T_{y}^{*}(S) .
\end{aligned}
$$

In particular, $E^{r,+} \cup Z^{r} \subset E^{l,+}$.

Proof. From the properties of the weight function in Section 3.1, and more precisely (3.19)-(3.20) that follow from them, we have

$$
\begin{aligned}
\mu^{l}= & \beta^{l}\left(x ; \xi_{0}, \eta\right)-\gamma(x)\left(\beta^{r}\left(x ; \xi_{0}, \eta\right)+\beta_{\varphi}^{r}\left(\mathrm{x} ; \xi_{0}, \eta\right) /\left(\partial_{x_{n}} \varphi^{r}\right)^{2}\right)+\beta_{\varphi}^{l}\left(\mathrm{x} ; \xi_{0}, \eta\right) /\left(\partial_{x_{n}} \varphi^{l}\right)^{2} \\
& +\gamma(x) \alpha^{r}\left(x ; \varphi^{r \prime}\right)-\alpha^{l}\left(x ; \varphi^{l \prime}\right)+\gamma(x) \mu^{r} \\
\geq & C\left\langle\left(\xi_{0}, \eta\right)\right\rangle^{2}+\gamma(x) \mu^{r} .
\end{aligned}
$$

Proposition 3.5. For the weight function $\varphi$ with the properties of Section 3.1 we have

$$
\operatorname{Char}\left(p_{\varphi}^{s}\right) \subset \operatorname{Char}\left(\operatorname{Re} p_{\varphi}^{s}\right) \subset E^{l,-} \cap\left\{x_{n}=0\right\} .
$$


Proof. From (3.11) we see that $\operatorname{Re} p_{\varphi}^{s}=0$ implies

$$
|\eta|_{g}+\left|\xi_{0}\right| \leq C\left(\left|\partial_{x_{0}} \varphi^{r / l}\right|+\left|d_{y} \varphi^{r / l}\right|_{g}\right)_{x_{n}=0}
$$

and we find

$$
\begin{aligned}
& \mu_{\mid x_{n}=0^{+}}^{l} \\
& =\left.\left[\left(\xi_{0}^{2}-\left(\partial_{x_{0}} \varphi\right)^{2}\right)\left(\frac{1}{c^{l}}-\frac{1}{c^{s}}\right)-\left(\partial_{x_{n}} \varphi^{l}\right)^{2}+\frac{1}{\left(\partial_{x_{n}} \varphi\right)^{2}}\left(\frac{\xi_{0} \partial_{x_{0}} \varphi}{c^{l}}+\tilde{r}\left(x ; \eta, d_{y} \varphi_{\mid x_{n}=0}\right)\right)^{2}\right]\right|_{x_{n}=0} .
\end{aligned}
$$

Using (3.21) together with (3.17) in this expression gives

$$
\mu_{\mid x_{n}=0^{+}}^{l} \leq\left.\left[-\left(\partial_{x_{n}} \varphi^{l}\right)^{2}+C v \inf \left(\left(\partial_{x_{n}} \varphi^{l}\right)^{2}\right)\right]\right|_{x_{n}=0} .
$$

The result thus follows when taking $v$ sufficiently small.

\subsection{Root properties}

The following lemma describes the position of the roots of $p_{\varphi}^{r / l}$ of (3.10) viewed as a second-order polynomial in $\xi_{n}$. The proof is given in Appendix C.4.

Lemma 3.6. We have the following root properties:

1. In the region $E^{r / l,+}$, the polynomial $p_{\varphi}^{r / l}$ defined in (3.10) has two distinct roots that satisfy $\operatorname{Im} \rho^{r / l,+}>0$ and $\operatorname{Im} \rho^{r / l,-}<0$. Moreover,

$$
\mu^{r / l} \geq C>0 \Leftrightarrow \operatorname{Im} \rho^{r / l,+} \geq C^{\prime}>0 \text { and } \operatorname{Im} \rho^{r / l,-} \leq-C^{\prime}<0 .
$$

2. In the region $E^{r l l,-}$, the imaginary parts of the two roots have the sign of $-\partial_{x_{n}} \varphi^{r / l}$.

3. In the region $Z^{r / l}$, one of the roots is real.

Moreover, there exist $C>0$ and $H>0$ such that $\left|\rho^{r / l,+}-\rho^{r / l,-}\right| \geq\left|\operatorname{Im} \rho^{r / l,+}-\operatorname{Im} \rho^{r / l,-}\right| \geq$ $C>0$ in the region $\left\{\mu^{r / l} \geq-H\right\}$.

Remark 3.7. Note that $\left(x, \xi_{0}, \eta\right) \in E^{r / l,+}$ for $\left|\xi_{0}\right|+|\eta|_{g}$ sufficiently large, say $\left|\xi_{0}\right|+$ $|\eta|_{g} \geq R$, uniformly in $\mathrm{x} \in\left[0, X_{0}\right] \times \overline{V_{\varepsilon}^{+}}$and for $h$ bounded. Note also that in the region $\left\{\mu^{r / l} \geq-H\right\}$, the roots $\rho^{r / l, \pm}$ are smooth since they do not cross.

For the polynomial $p_{\varphi}^{r}$, for $\left|\xi_{0}\right|+|\eta|_{g}$ small, i.e. in the region $E^{r,-}$, the two roots $\rho^{r,+}$ and $\rho^{r,-}$ both have negative imaginary parts. As the value of $\mu^{r}$ increases, the root $\rho^{r,+}$ moves towards the real axis, and crosses it in $Z^{r}$. In $E^{r,+}$ we have $\operatorname{Im} \rho^{r,+}>0$ and $\operatorname{Im} \rho^{r,-}<0$.

For the polynomial $p_{\varphi}^{l}$, for $\left|\xi_{0}\right|+|\eta|_{g}$ small, i.e. in $E^{l,-}$, the two roots $\rho^{l,+}$ and $\rho^{l,-}$ both have positive imaginary parts. As the value of $\mu^{l}$ increases, the root $\rho^{l,-}$ moves towards the real axis, and crosses it in $Z^{l}$. In $E^{l,+}$ we have $\operatorname{Im} \rho^{l,+}>0$ and $\operatorname{Im} \rho^{l,-}<0$. The "motion" of the roots of $p_{\varphi}^{l}$ and $p_{\varphi}^{r}$ is illustrated in Figure 3. 

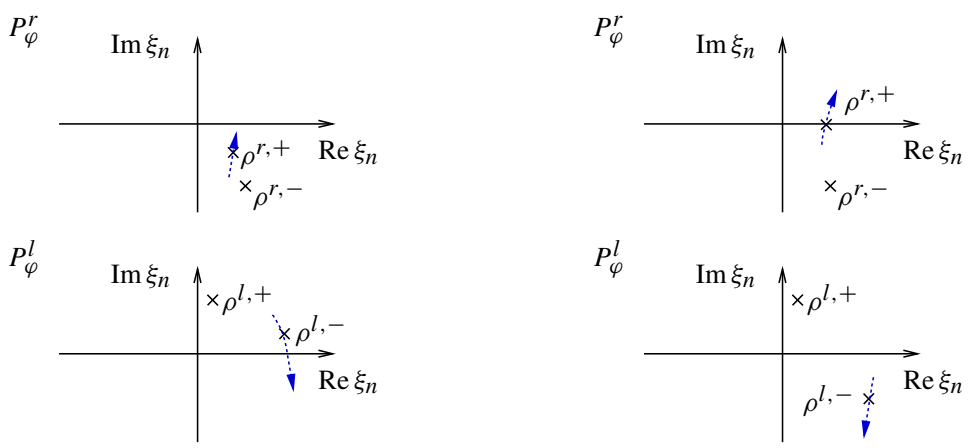

$P_{\varphi}^{l}$

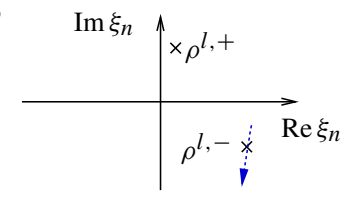

(a) Root configuration in $E^{r,-}, \mu^{d}<0$

(b) Root configuration in $Z^{r}, \mu^{r}=0$

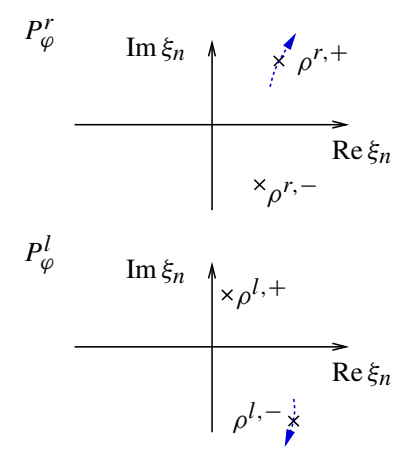

(c) Root configuration in $E^{r,+}, \mu^{r}>0$

Fig. 3. The root $\rho^{r,+}$ crosses the real axis before the root $\rho^{l,-}$ does, as $\mu^{r}$ decreases.

We now define

$$
\mathcal{M}_{+}=\left(0, X_{0}\right) \times S \times[0,2 \varepsilon] .
$$

We also set

$$
\begin{aligned}
\mathcal{M}_{+}^{*} & :=\left\{\left(x_{0}, y, x_{n}, \xi_{0}, \eta\right) \in\left(0, X_{0}\right) \times S \times[0,2 \varepsilon] \times \mathbb{R} \times T_{y}^{*}(S)\right\} \\
& \simeq T^{*}\left(\left(0, X_{0}\right) \times S\right) \times[0,2 \varepsilon] .
\end{aligned}
$$

With the symbols defined in Section B.2 (see Definition B.4) we obtain the following result.

Lemma 3.8. Let $H$ be as given in Lemma 3.6. Let $\chi^{r / l} \in S_{\mathcal{T}}^{0}\left(\mathcal{M}_{+}^{*}\right)$ with support in $\left\{\mu^{r / l} \geq-H\right\}$. Then $\chi^{r / l} \rho^{r / l}, \pm \in S_{\mathcal{T}}^{1}\left(\mathcal{M}_{+}^{*}\right)$. Let $C_{0}>0$. There exists $C>0$ such that $\left|\operatorname{Im} \rho^{r / l, \pm}\right| \geq C\left(1+\left|\xi_{0}\right|+|\eta|_{g}\right)$ in $\left\{\mu^{r / l} \geq C_{0}\right\}$. It follows that for some $C^{\prime}>0$ we have

$$
\left|\rho^{r / l,+}-\rho^{r / l,-}\right| \geq\left|\operatorname{Im}\left(\rho^{r / l,+}-\rho^{r / l,-}\right)\right| \geq C^{\prime}\left(1+\left|\xi_{0}\right|+|\eta|_{g}\right) \quad \text { in }\left\{\mu^{r / l} \geq C_{0}\right\} .
$$

We refer to Appendix C.5 for a proof. 


\subsection{Microlocalization operators}

We define the following open sets in (tangential) phase space:

$$
\begin{aligned}
& \mathscr{E}=\left\{\left(\mathrm{x}, \xi_{0}, \eta\right) \in \mathcal{M}_{+}^{*} ; \epsilon_{1}<\mu^{r}\left(\mathrm{x}, \xi_{0}, \eta\right)\right\}, \\
& \mathscr{Z}=\left\{\left(\mathrm{x}, \xi_{0}, \eta\right) \in \mathcal{M}_{+}^{*} ;-2 \epsilon_{1}<\mu^{r}\left(\mathrm{x}, \xi_{0}, \eta\right)<2 \epsilon_{1}\right\}, \\
& \mathscr{F}=\left\{\left(\mathrm{x}, \xi_{0}, \eta\right) \in \mathcal{M}_{+}^{*} ; \epsilon_{2}<\mu^{l}\left(\mathrm{x}, \xi_{0}, \eta\right) \text { and } \mu^{r}\left(\mathrm{x}, \xi_{0}, \eta\right)<-\epsilon_{1}\right\}, \\
& \mathscr{G}=\left\{\left(\mathrm{x}, \xi_{0}, \eta\right) \in \mathcal{M}_{+}^{*} ; \mu^{l}\left(\mathrm{x}, \xi_{0}, \eta\right)<2 \epsilon_{2}\right\} .
\end{aligned}
$$

The constants $\epsilon_{1}$ and $\epsilon_{2}$ are taken such that $\sup (\gamma) \epsilon_{1}+\epsilon_{2}<C_{0} / 2$, with $C_{0}$ as in Proposition 3.4. Our analysis in the region $\mathscr{Z}$ will require $\epsilon_{1}$ to be small (see Section 4.4 below). Recall that $\gamma$ is defined in Section 3.1. This yields $\overline{\mathscr{G}} \cap \overline{\mathscr{Z}}=\emptyset$. As a consequence of Propositions 3.4 and 3.5, the position of the different microlocal zones can be represented as in Figure 4. In particular, $\operatorname{Char}\left(p_{\varphi}^{s}\right) \subset(\mathscr{G} \backslash \mathscr{F}) \cap\left\{x_{n}=0\right\}$.

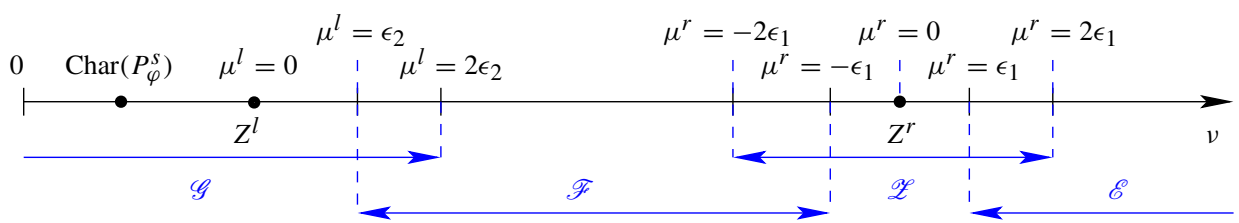

Fig. 4. Sketch of the relative position of the different phase-space regions. Here, $\left(x, \xi_{0}, \eta\right)$ is fixed and we plot the different zones for $\left(\mathrm{x}, v \xi_{0}, v \eta\right)$ as $v$ increases from 0 to $\infty$, with $v$ representing the norm of the tangential frequencies. This situation can be represented in this form since for $\mathrm{x}$ fixed, the sets $E^{r / l},-$ and $\left\{p_{\varphi}^{s} \leq 0\right\}$ are star-shaped with respect to 0 in the variables $\left(\xi_{0}, \eta\right) \in$ $T_{\left(x_{0}, y\right)}^{*}\left(\left(0, X_{0}\right) \times S\right)$.

We introduce a $\mathscr{C}^{\infty}$ partition of unity subordinated to the open covering of $\mathcal{M}_{+}^{*}$ by $\mathscr{E}, \mathscr{Z}, \mathscr{F}$ and $\mathscr{G}$,

$$
\chi_{\mathscr{E}}+\chi_{\mathscr{Z}}+\chi_{\mathscr{F}}+\chi_{\mathscr{G}}=1, \quad 0 \leq \chi_{\bullet} \leq 1, \operatorname{supp}\left(\chi_{\bullet}\right) \subset \bullet \bullet \bullet=\mathscr{E}, \mathscr{Z}, \mathscr{F}, \mathscr{G} .
$$

The sets $\mathscr{Z}, \mathscr{F}$ and $\mathscr{G}$ are relatively compact, which gives $\chi_{\mathscr{Z}}, \chi_{\mathscr{F}}, \chi_{\mathscr{G}} \in S_{\mathcal{T}}^{-\infty}\left(\mathcal{M}_{+}^{*}\right)$ and consequently $\chi_{\mathscr{E}} \in S_{\mathcal{T}}^{0}\left(\mathcal{M}_{+}^{*}\right)$. Associated with these symbols, we now define tangential pseudo-differential operators on $\mathcal{M}_{+}$.

Given $0<\alpha_{0}<X_{0} / 2$, we choose a function $\zeta^{1} \in \mathscr{C}_{c}^{\infty}\left(0, X_{0}\right)$ that satisfies $\zeta^{1}=1$ on a neighborhood of $\left(\alpha_{0}, X_{0}-\alpha_{0}\right)$ and $0 \leq \zeta^{1} \leq 1$. Setting

$$
\zeta_{j}\left(x_{0}, y, x_{n}\right)=\zeta^{1}\left(x_{0}\right) \psi_{j}(y)
$$

gives a partition of unity on $\left(\alpha_{0}, X_{0}-\alpha_{0}\right) \times S \times[0,2 \varepsilon]$. Recall that $\left(\psi_{j}\right)_{j \in J}$ is a partition of unity on $S$ (see Section 1.4.3).

We define the following operators on $\mathcal{M}_{+}$:

$$
\Xi_{\bullet}=\sum_{j \in J} \Xi_{\bullet}, j \quad \text { with } \quad \Xi_{\bullet}, j=\phi_{j}^{*} \operatorname{Op}_{\mathcal{T}}\left(\chi_{\bullet}, j\right)\left(\phi_{j}^{-1}\right)^{*} \zeta_{j}, j \in J, \bullet=\mathscr{E}, \mathscr{Z}, \mathscr{F}, \mathscr{G},
$$


where $\phi_{j}^{*}$ denotes the pullback by the function $\phi_{j}$ and

$$
\chi_{\bullet, j}=\tilde{\zeta}_{j}\left(\phi_{j}^{-1}\right)^{*} \chi_{\bullet}
$$

and $\tilde{\zeta}_{j}$ denotes a function in $\mathscr{C}_{c}^{\infty}\left(\left(0, X_{0}\right) \times \tilde{U}_{j}\right)$ with $\tilde{\zeta}_{j}=1$ in a neighborhood of $\operatorname{supp}\left(\left(\phi_{j}^{-1}\right)^{*} \zeta_{j}\right)$

Proposition B.14 in Appendix B.3 shows that the operators $\Xi_{\bullet}$ are zero-order tangential semiclassical operators on $\mathcal{M}_{+}$, with principal symbol $\zeta^{1}\left(x_{0}\right) \chi_{\bullet}\left(\mathrm{x}, \xi_{0}, \eta\right)$.

Remark 3.9. The role of the parameter $\alpha_{0}$ introduced here is to avoid considering boundary problems on $\left(\{0\} \cup\left\{X_{0}\right\}\right) \times S \times[0,2 \varepsilon]$.

\section{Proof of the Carleman estimate in a neighborhood of the interface}

In this section, we prove Carleman estimates in the four microlocal regions described above, that is, for functions $\Xi_{\bullet} v^{r / l}$, with $v^{r / l} \in \mathscr{C}_{c}^{\infty}\left(\left(0, X_{0}\right) \times S \times[0,2 \varepsilon)\right)$ and $\bullet=$ $\mathscr{E}, \mathscr{Z}, \mathscr{F}, \mathscr{G}$. It will be more convenient to do this in local coordinates, ${ }^{4}$ since we can then use the techniques and some of the results of [LR10].

Our strategy in each microlocal region $\bullet$ (with $\bullet=\mathscr{E}, \mathscr{Z}, \mathscr{F}, \mathscr{G}$ ) is hence the following: We first produce Carleman estimates in each local chart $\left(0, X_{0}\right) \times \tilde{U}_{j} \times[0,2 \varepsilon)$ for the functions

$$
u_{\bullet, j}^{r / l}:=\mathrm{Op}_{\mathcal{T}}\left(\chi_{\bullet, j}\right) v_{j}^{r / l} \quad \text { and } \quad u_{\bullet, j}^{s}:=\mathrm{Op}_{\mathcal{T}}\left(\chi_{\bullet, j_{\mid x_{n}=0^{+}}}\right) v_{j}^{s},
$$

where

$$
v_{j}^{r / l}:=\left(\phi_{j}^{-1}\right)^{*} \zeta_{j} v^{r / l} \quad \text { and } \quad v_{j}^{s}:=\left(\phi_{j}^{-1}\right)^{*} \zeta_{j} v^{s},
$$

with $\zeta_{j}$ defined in (3.23). Then, we pull the local estimates back to the manifold and patch them together to finally obtain a Carleman estimate for $\Xi_{\bullet} v^{r / l}$, as

$$
\Xi_{\bullet} v^{r / l}=\sum_{j} \phi_{j}^{*} u_{\bullet, j}^{r / l} .
$$

Note that the functions $v_{j}^{r / l}$ (resp. $\left.v_{j}^{s}\right)$ are defined in $\left(0, X_{0}\right) \times \tilde{U}_{j} \times[0,2 \varepsilon)$ (resp. $\left.\left(0, X_{0}\right) \times \tilde{U}_{j}\right)$. Yet, because of their compact support, we naturally extend them by zero to $\mathbb{R} \times \mathbb{R}^{n-1} \times \mathbb{R}^{+}$(resp. $\mathbb{R} \times \mathbb{R}^{n-1}$ ). In the following, functions with such a compact support will be extended similarly.

We shall use the notation $\lesssim$ for $\leq C$, with a constant $C$ independent of $\delta$ and $h$ (but depending on $\delta_{0}$ and $h_{0}$ ).

\footnotetext{
4 However, note that it would be interesting to obtain the results of [LR10] directly in a global setting.
} 


\subsection{Preliminary observations}

In the local chart $\tilde{U}_{j}$, the differential operators $P_{\varphi}^{\alpha}, \alpha=r, l$ or $s$, are given by

$$
P_{\varphi, j}^{\alpha}=\left(\phi_{j}^{-1}\right)^{*} P_{\varphi}^{\alpha} \phi_{j}^{*},
$$

with principal symbol $p_{\varphi, j}^{\alpha}=\left(\phi_{j}^{-1}\right)^{*} p_{\varphi}^{\alpha}$.

Observe that the definition of $\mu^{r / l}$ in (3.12), and of the associated microlocal regions $Z^{r / l}, E^{r / l, \pm}$ in (3.13)-(3.14), and $\mathscr{E}, \mathscr{Z}, \mathscr{F}$ and $\mathscr{G}$ in (3.22), are geometrically invariant.

In local coordinates, System (3.9) becomes

$$
\left\{\begin{array}{rlrl}
P_{\varphi, j}^{r / l} v_{j}^{r / h}= & \tilde{F}_{\varphi, j}^{r / l} & & \text { in }\left(0, X_{0}\right) \times \tilde{U}_{j} \times[0,2 \varepsilon), \\
P_{\varphi, j}^{s} v_{j}^{s}= & \frac{h i}{c_{j}^{s} \delta}\left(c_{j}^{r}\left(D_{x_{n}}+i \partial_{x_{n}} \varphi_{j}^{r}\right) v_{j \mid x_{n}=0^{+}}^{r}\right. & & \\
& \left.+\left.c_{j}^{l}\left(D_{x_{n}}+i \partial_{x_{n}} \varphi_{j}^{l}\right) v_{j}^{l}\right|_{x_{n}=0^{+}}+\tilde{\Theta}_{\varphi, j}^{s}\right) & & \text { in }\left(0, X_{0}\right) \times \tilde{U}_{j}, \\
v_{j}^{r / l}{ }_{\mid x_{n}=0^{+}}=v_{j}^{s}+\theta_{\varphi, j}^{r / l} & & \text { in }\left(0, X_{0}\right) \times \tilde{U}_{j},
\end{array}\right.
$$

where we have set

$$
\left\{\begin{array}{l}
\tilde{F}_{\varphi, j}^{r / l}=\left(\phi_{j}^{-1}\right)^{*} \zeta_{j} F_{\varphi}^{r / l}+\left(\phi_{j}^{-1}\right)^{*}\left[P_{\varphi}^{r / l}, \zeta_{j}\right] v^{r / l}, \\
\theta_{\varphi, j}^{\prime / l}=\left(\phi_{j}^{-1}\right)^{*} \zeta_{j} \theta_{\varphi}^{r / l}, \\
\Theta_{\varphi, j}^{s}=\left(\phi_{j}^{-1}\right)^{*} \zeta_{j} \Theta_{\varphi}^{s}, \quad \tilde{\Theta}_{\varphi, j}^{s}=\Theta_{\varphi, j}^{s}+\frac{c_{j}^{s} \delta}{h i}\left(\phi_{j}^{-1}\right)^{*}\left[P_{\varphi}^{s}, \zeta_{j}\right] v^{s} \\
\varphi_{j}^{r / l}=\left(\phi_{j}^{-1}\right)^{*} \varphi^{r / l}, \quad c_{j}^{r / l}=\left(\phi_{j}^{-1}\right)^{*} c^{r / l}, \quad c_{j}^{s}=\left(\phi_{j}^{-1}\right)^{*} c^{s}
\end{array}\right.
$$

with $\left[P_{\varphi}^{r / h}, \zeta_{j}\right] \in h \mathscr{D}^{1}\left(\mathcal{M}_{+}\right)$and $\left[P_{\varphi}^{s}, \zeta_{j}\right] \in h \mathscr{D}_{\mathcal{T}}^{1}\left(\mathcal{M}_{+}\right)$.

We now formulate System (4.3) in terms of $u_{\bullet}, j$ in preparation for the estimations in the four different microlocal zones. First, we have

$$
P_{\varphi, j}^{r / l} u_{\bullet}^{r / l},=\operatorname{Op} \mathcal{T}\left(\chi_{\bullet, j}\right) P_{\varphi, j}^{r / l} v_{j}^{r / l}+\underbrace{\left[P_{\varphi, j}^{r / l}, \operatorname{Op} \mathcal{T}\left(\chi_{\bullet}\right)\right]}_{\in h\left(\Psi_{\mathcal{T}}^{0} D_{x_{n}}+\Psi_{\mathcal{T}}^{1}\right)} v_{j}^{r / l} .
$$

In particular, this gives

$$
\left\|P_{\varphi, j}^{r / h} u_{\bullet, j}^{r / l}\right\|_{0} \lesssim\left\|P_{\varphi, j}^{r / l} v_{j}^{r / l}\right\|_{0}+h\left\|v_{j}^{r / h}\right\|_{1} .
$$

Second, as a consequence of (4.3), the transmission conditions satisfied by $u_{\bullet, j}^{r / h}$ and $u_{\bullet, j}^{s}$ read

$\left\{\begin{array}{l}\frac{\delta c_{j}^{s}}{h i} P_{\varphi, j}^{s} u_{\bullet, j}^{s}=\left(c_{j}^{l}\left(D_{x_{n}}+i \partial_{x_{n}} \varphi_{j}^{l}\right) u_{\bullet, j}^{l}\right)_{\mid x_{n}=0^{+}}+\left(c_{j}^{r}\left(D_{x_{n}}+i \partial_{x_{n}} \varphi_{j}^{r}\right) u_{\bullet, j}^{r}\right)_{\mid x_{n}=0^{+}}+G_{1}, \\ u_{\bullet, j \mid x_{n}=0^{+}}^{r / h}=u_{\bullet, j}^{s}+\theta_{\bullet}^{r / l},\end{array}\right.$

with $\theta_{\bullet, j}^{r / l}=\mathrm{Op}_{\mathcal{T}}\left(\left.\chi_{\bullet, j}\right|_{\mid x_{n}=0^{+}}\right) \theta_{\varphi, j}^{r / l}$ and 


$$
\begin{aligned}
G_{1}= & \frac{\delta c_{j}^{s}}{h i} \underbrace{\left[P_{\varphi, j}^{s}, \operatorname{Op} \mathcal{T}\left(\chi_{\bullet, j} j_{\mid x_{n}=0^{+}}\right)\right]}_{\in h \Psi_{\mathcal{T}}^{1}} v_{j}^{s}+\underbrace{\left[\mathrm{Op} \mathcal{T}\left(\chi_{\bullet, j} j_{\mid x_{n}=0^{+}}\right), c_{j}^{l}\left(D_{x_{n}}+i \partial_{x_{n}} \varphi_{j}^{l}\right)\right]}_{\in h \Psi_{\mathcal{T}}^{0}} v_{j_{\mid x_{n}=0^{+}}^{l}}^{l} \\
& +\underbrace{\left[\mathrm{Op} \mathcal{T}\left(\left.\chi_{\bullet, j}\right|_{x_{n}=0^{+}}\right), c_{j}^{r}\left(D_{x_{n}}+i \partial_{x_{n}} \varphi_{j}^{r}\right)\right]}_{\in h \Psi_{\mathcal{T}}^{0}} v_{\left.\left.\right|_{\mid x_{n}=0^{+}} ^{r}+\mathrm{Op}_{\mathcal{T}}\left(\left.\chi_{\bullet, j}\right|_{\mid x_{n}=0^{+}}\right)\right) \tilde{\Theta}_{\varphi, j}^{s} .}^{s}
\end{aligned}
$$

We have the estimate

$$
\begin{aligned}
\left|G_{1}\right|_{0} & \lesssim \delta\left|v_{j}^{s}\right|_{1}+h\left|v_{j_{\mid x_{n}=0^{+}}}^{l}\right|_{0}+h\left|v_{j_{\mid x_{n}=0^{+}}^{r}}\right|_{0}+\left|\tilde{\Theta}_{\varphi, j}^{s}\right|_{0} \\
& \lesssim(\delta+h)\left|v_{j}^{s}\right|_{1}+h\left|\theta_{\varphi, j}^{l}\right|_{0}+h\left|\theta_{\varphi, j}^{r}\right|_{0}+\left|\Theta_{\varphi, j}^{s}\right|_{0},
\end{aligned}
$$

by (4.3) and (4.4). We set

$$
\gamma_{0}\left(u_{\bullet}^{r / h}\right)=\left.u_{\bullet, j}^{r / l}\right|_{x_{n}=0^{+}}, \quad \gamma_{1}\left(u_{\bullet}^{r / h}\right)=\left(D_{x_{n}} u_{\bullet}^{r / h}\right)_{\mid x_{n}=0^{+}} .
$$

In this local setting we also introduce

$$
\begin{aligned}
& \beta=\left(c_{j}^{r} / c_{j}^{l}\right)_{\mid x_{n}=0^{+}}, \quad \tilde{G}_{1}=i \partial_{x_{n}} \varphi_{j}^{l}\left(\theta_{\bullet, j}^{l}-\theta_{\bullet, j}^{r}\right)+\frac{1}{c_{j \mid x_{n}=0^{+}}^{l}} G_{1}, \\
& k=-i\left(\partial_{x_{n}} \varphi_{j \mid x_{n}=0^{+}}^{l}+\beta \partial_{x_{n}} \varphi_{j \mid x_{n}=0^{+}}^{r}\right) .
\end{aligned}
$$

Transmission conditions (4.6) can be written as

$$
\left\{\begin{array}{l}
\frac{\delta c_{j}^{s}}{h i c_{j}^{l}} P_{\varphi, j}^{s} u_{\bullet, j}^{s}=\gamma_{1}\left(u_{\bullet, j}^{l}\right)+\beta \gamma_{1}\left(u_{\bullet}^{r}\right)-k \gamma_{0}\left(u_{\bullet, j}^{r}\right)+\tilde{G}_{1}, \\
\gamma_{0}\left(u_{\bullet, j}^{r / l}\right)=u_{\bullet, j}^{s}+\theta_{\bullet}^{r / l},
\end{array}\right.
$$

where the remainder $\tilde{G}_{1}$ can be estimated thanks to (4.7) by

$$
\left|\tilde{G}_{1}\right|_{0} \lesssim(\delta+h)\left|v_{j}^{s}\right|_{1}+\left|\Theta_{\varphi, j}^{s}\right|_{0}+\left|\theta_{\varphi, j}^{l}\right|_{0}+\left|\theta_{\varphi, j}^{r}\right|_{0} .
$$

We are now prepared to prove the different Carleman estimates in the four microlocal regions.

\subsection{Estimate in the region $\mathscr{G}$}

Here, we prove a Carleman estimate for $u_{\mathscr{G}, j}$, and consequently for $\Xi_{\mathscr{G} V}$.

We introduce a microlocal cut-off function $\chi_{\mathscr{G} \mathscr{F}} \in \mathscr{C}_{c}^{\infty}\left(\mathcal{M}_{+}^{*}\right), 0 \leq \chi \mathscr{G} \mathscr{F} \leq 1$, satisfying

$$
\begin{array}{ll}
\chi \mathscr{G} \mathscr{F}=1 & \text { on a neighborhood of } \operatorname{supp}(\chi \mathscr{G}), \\
\chi \mathscr{G}+\chi_{\mathscr{F}}=1 & \text { on a neighborhood of } \operatorname{supp}\left(\chi_{\mathscr{G} \mathscr{F}}\right) .
\end{array}
$$

We choose $\zeta^{2} \in \mathscr{C}_{c}^{\infty}\left(0, X_{0}\right)$ such that $0 \leq \zeta^{2} \leq 1, \zeta^{2}=1$ on a neighborhood of $\operatorname{supp}\left(\zeta^{1}\right)$ (with $\zeta^{1}$ defined in (3.23)), and such that $\tilde{\zeta}_{j}=1$ on $\operatorname{supp}\left(\left(\phi_{j}^{-1}\right)^{*} \zeta_{j}^{2}\right)$ where 
$\zeta_{j}^{2}\left(x_{0}, y\right)=\zeta^{2}\left(x_{0}\right) \psi_{j}(y)$. As in (3.25) we set

$$
\chi \mathscr{G F}, j=\tilde{\zeta}_{j}\left(\phi_{j}^{-1}\right)^{*} \chi \mathscr{G} \mathscr{F},
$$

and we define the associated tangential pseudo-differential operator $\Xi_{\mathscr{G} \mathscr{F}}$ by

$$
\Xi_{\mathscr{G} \mathscr{F}}=\sum_{j \in J} \Xi_{\mathscr{G} \mathscr{F}, j} \quad \text { with } \quad \Xi_{\mathscr{G} \mathscr{F}, j}=\phi_{j}^{*} \mathrm{Op}_{\mathcal{T}}(\chi \mathscr{G} \mathscr{F}, j)\left(\phi_{j}^{-1}\right)^{*} \zeta_{j}^{2}, j \in J,
$$

Note that the local symbol (see Proposition B.7) of $\Xi_{\mathscr{G} \mathscr{F}}$ in each chart is equal to 1 in the support of the local symbol of $\Xi_{\mathscr{G}}$.

We recall that the function $\zeta=\zeta\left(x_{n}\right) \in \mathscr{C}_{c}^{\infty}([0,2 \varepsilon))$ satisfies $\zeta(0)=1$ on $[0, \varepsilon)$.

Making use of the Calderón projector technique for $P_{\varphi, j}^{r}$ and of the standard Carleman techniques for $P_{\varphi, j}^{l}$, we obtain the following partial estimate.

Proposition 4.1. Suppose that the weight function $\varphi$ satisfies the properties listed in Section 3.1. Then, for all $\delta_{0}>0$, there exist $C>0$ and $h_{0}>0$ such that, for all $0<\delta \leq \delta_{0}$ and $0<h \leq h_{0}$, and for $v^{r / l} \in \mathscr{C}_{c}^{\infty}\left(\left(0, X_{0}\right) \times S \times[0,2 \varepsilon)\right)$ and $v^{s} \in \mathscr{C}_{c}^{\infty}\left(\left(0, X_{0}\right) \times S\right)$ satisfying (3.9), we have

$$
\begin{aligned}
\left\|\Xi_{\mathscr{G}} v^{r}\right\|_{1}^{2}+h\left|\Xi_{\mathscr{G}} v_{\mid x_{n}=0^{+}}^{r}\right|_{1}^{2} & +h\left|D_{x_{n}} \Xi_{\mathscr{G}} v_{\mid x_{n}=0^{+}}^{r}\right|_{0}^{2} \\
& \leq C\left(\left\|P_{\varphi}^{r} v^{r}\right\|_{0}^{2}+h^{2}\left\|v^{r}\right\|_{1}^{2}+h^{4}\left|D_{x_{n}} v_{\mid x_{n}=0^{+}}^{r}\right|_{0}^{2}\right),
\end{aligned}
$$

and

$$
\begin{aligned}
h\left\|\Xi_{\mathscr{G}} v^{l}\right\|_{1}^{2}+h\left|\Xi_{\mathscr{G}} v_{\mid x_{n}=0^{+}}^{l}\right|_{1}^{2}+h\left|D_{x_{n}} \boldsymbol{\Xi}_{\mathscr{G}} v_{\mid x_{n}=0^{+}}^{l}\right|_{0}^{2} \\
\leq C\left(1+\frac{\delta^{2}}{h^{2}}\right)\left(\left\|\zeta P_{\varphi}^{r} v^{r}\right\|_{0}^{2}+h^{2}\left\|\Xi_{\mathscr{G} \mathscr{F}} v^{r}\right\|_{1}^{2}+h^{4}\left|D_{x_{n}} v_{\mid x_{n}=0^{+}}^{r}\right|_{0}^{2}+h^{4}\left\|v^{r}\right\|_{1}^{2}+h^{3}\left|v^{s}\right|_{1}^{2}\right) \\
\quad+C\left(\left\|P_{\varphi}^{l} v^{l}\right\|_{0}^{2}+h^{2}\left\|v^{l}\right\|_{1}^{2}+h\left|\theta_{\varphi}^{l}\right|_{1}^{2}+\frac{\delta^{2}}{h}\left|\theta_{\varphi}^{r}\right|_{0}^{2}+h\left|\theta_{\varphi}^{r}\right|_{1}^{2}+h\left|\Theta_{\varphi}^{s}\right|_{0}^{2}\right) .
\end{aligned}
$$

Proof. The function $u_{\mathscr{G}}, j$, defined in (4.1), satisfies $\left(\mathrm{TC}_{\bullet, j}\right)$, with $\bullet=\mathscr{G}$. On the " $r$ " side, the root configuration described in Lemma 3.6 (and represented in Figure 3) allows us to apply the Calderón projector technique used in [LR97, LR10]. According to [LR10, Remark 2.5] and using (2.59)-(2.61) therein, applied with $v^{d}$ replaced here by $v_{j}^{r}$, we have

$$
\begin{aligned}
\left\|u_{\mathscr{G}, j}^{r}\right\|_{1}+h^{1 / 2}\left|\gamma_{0}\left(u_{\mathscr{G}, j}^{r}\right)\right|_{1}+ & h^{1 / 2}\left|\gamma_{1}\left(u_{\mathscr{G}_{, j}}^{r}\right)\right|_{0} \\
& \lesssim\left\|P_{\varphi, j}^{r} v_{j}^{r}\right\|_{0}+h\left\|v_{j}^{r}\right\|_{1}+\left.h^{2}\left|D_{x_{n}} v_{j}^{r}\right|_{x_{n}=0^{+}}\right|_{0},
\end{aligned}
$$

which is a local version of (4.13).

Let us now explain how such local estimates can be patched together to yield (4.13). Concerning the first term on the left-hand side of (4.15), and using the definition of Sobolev norms given in (1.20)-(1.22), we have

$$
\left\|\Xi_{\mathscr{G}} v^{r}\right\|_{1} \lesssim \sum_{j \in J}\left\|u_{\mathscr{G}, j}^{r}\right\|_{1}, \quad\left|\Xi_{\mathscr{G}} v^{r} x_{n}=0^{+}\right|_{1} \lesssim \sum_{j \in J}\left|\gamma_{0}\left(u_{\mathscr{G}, j}^{r}\right)\right|_{1}
$$


by (4.2) and Lemma B.15. Similarly we have $D_{x_{n}} \Xi_{\mathscr{G}} v_{\mid x_{n}=0^{+}}^{r}=\sum_{j} \phi_{j}^{*} \gamma_{1}\left(u_{\mathscr{G}, j}^{r}\right)$ since $\phi_{j}^{*}$ does not depend on the $x_{n}$ variable. As a consequence, we obtain

$$
\left|\left(D_{x_{n}} \Xi_{\mathscr{G}} v^{r}\right)_{\mid x_{n}=0^{+}}\right|_{0} \leq \sum_{j \in J}\left|\phi_{j}^{*} \gamma_{1}\left(u_{\mathscr{G}, j}^{r}\right)\right|_{0} \lesssim \sum_{j \in J}\left|\gamma_{1}\left(u_{\mathscr{G}, j}^{r}\right)\right|_{0},
$$

by Lemma 1.9 .

Now concerning the right-hand side of (4.15), we directly have

$$
\left\|v_{j}^{r}\right\|_{1}=\left\|\left(\phi_{j}^{-1}\right)^{*} \zeta_{j} v^{r}\right\|_{1}=\left\|\zeta^{1}\left(\phi_{j}^{-1}\right)^{*} \psi_{j} v^{r}\right\|_{1} \lesssim\left\|\left(\phi_{j}^{-1}\right)^{*} \psi_{j} v^{r}\right\|_{1} \lesssim\left\|v^{r}\right\|_{1},
$$

by the definition of $\|\cdot\|_{1}$ on $\mathcal{M}_{+}$, as well as

$$
\left|D_{x_{n}} v_{j}^{r}{\mid x_{n}=0^{+}}_{0}\right|_{0} \lesssim\left|D_{x_{n}} v_{\mid x_{n}=0^{+}}^{r}\right|_{0} .
$$

Finally, we compute

$$
P_{\varphi, j}^{r} v_{j}^{r}=\left(\phi_{j}^{-1}\right)^{*} P_{\varphi}^{r} \phi_{j}^{*}\left(\phi_{j}^{-1}\right)^{*} \zeta_{j} v^{r}=\left(\phi_{j}^{-1}\right)^{*} \zeta_{j} P_{\varphi}^{r} v^{r}+\left(\phi_{j}^{-1}\right)^{*}\left[P_{\varphi}^{r}, \zeta_{j}\right] v^{r} .
$$

We have

$$
\left\|\left(\phi_{j}^{-1}\right)^{*} \zeta_{j} P_{\varphi}^{r} v^{r}\right\|_{0}=\left\|\zeta^{1}\left(\phi_{j}^{-1}\right)^{*} \psi_{j} P_{\varphi}^{r} v^{r}\right\|_{0} \leq\left\|P_{\varphi}^{r} v^{r}\right\|_{0}
$$

and, using Lemma 1.9,

$$
\left\|\left(\phi_{j}^{-1}\right)^{*}\left[P_{\varphi}^{r}, \zeta_{j}\right] v^{r}\right\|_{0} \lesssim\left\|\left[P_{\varphi}^{r}, \zeta_{j}\right] v^{r}\right\|_{0} \lesssim h\left\|v^{r}\right\|_{1},
$$

since $\left[P_{\varphi}^{r}, \zeta_{j}\right] \in h \mathscr{D}^{1}\left(\mathcal{M}_{+}\right)$. Finally combining all the estimates (4.16)-(4.21) with the local inequalities (4.15) summed over $j \in J$, we obtain the desired global estimate (4.13) on $\mathcal{M}_{+}$.

To obtain estimate (4.14) on the " $l$ " side we first need a more precise estimate for the " $r$ " side. For this, we introduce another microlocal cut-off function $\tilde{\chi}_{\mathscr{G} \mathscr{F}}$ satisfying the same requirements (4.12) as $\chi \mathscr{G \mathscr { F }}$, and such that $\chi \mathscr{G \mathscr { F }}=1$ on a neighborhood of $\operatorname{supp}(\tilde{\chi} \mathscr{G} \mathscr{F})$. We choose $\zeta^{3} \in \mathscr{C}_{c}^{\infty}\left(0, X_{0}\right)$ such that $0 \leq \zeta^{3} \leq 1, \zeta^{3}=1$ on a neighborhood of $\operatorname{supp}\left(\zeta^{1}\right)$, and such that $\zeta^{2}=1$ on a neighborhood of $\operatorname{supp}\left(\zeta^{3}\right)$. As in (3.25) we set

$$
\tilde{\chi}_{\mathscr{G} \mathscr{F}, j}=\tilde{\zeta}_{j}\left(\phi_{j}^{-1}\right)^{*} \tilde{\chi} \mathscr{G},
$$

and we define the associated tangential pseudo-differential operator $\tilde{\Xi}_{\mathscr{G} \mathscr{F}}$ by

$$
\tilde{\Xi}_{\mathscr{G} \mathscr{F}}=\sum_{j \in J} \tilde{\Xi}_{\mathscr{G} \mathscr{F}, j} \quad \text { with } \quad \tilde{\Xi}_{\mathscr{G} \mathscr{F}, j}=\phi_{j}^{*} \mathrm{Op}_{\mathcal{T}}\left(\tilde{\chi}_{\mathscr{G} \mathscr{F}, j}\right)\left(\phi_{j}^{-1}\right)^{*} \zeta_{j}^{3}, \zeta_{j}^{3}=\zeta^{3} \psi_{j}, j \in J,
$$

According to [LR10, Remark 2.5] and using (2.60) and (2.61) therein, applied with $v^{d}$ replaced by $\zeta\left(x_{n}\right)\left(\phi_{j}^{-1}\right)^{*} \zeta_{j} \tilde{\Xi}_{\mathscr{G} \mathscr{F}} v^{r}$, we have

$$
\begin{aligned}
& h^{1 / 2}\left|\gamma_{0}\left(\mathrm{Op}_{\mathcal{T}}\left(\chi \mathscr{G}_{, j}\right)\left(\phi_{j}^{-1}\right)^{*} \zeta_{j} \tilde{\Xi}_{\mathscr{G} \mathscr{F}} v^{r}\right)\right|_{1}+h^{1 / 2}\left|\gamma_{1}\left(\mathrm{Op}_{\mathcal{T}}\left(\chi \mathscr{G}_{, j}\right)\left(\phi_{j}^{-1}\right)^{*} \zeta_{j} \tilde{\Xi}_{\mathscr{G} \mathscr{F}} v^{r}\right)\right|_{0} \\
& \quad \lesssim\left\|P_{\varphi, j}^{r} \zeta\left(\phi_{j}^{-1}\right)^{*} \zeta_{j} \tilde{\Xi}_{\mathscr{G} \mathscr{F}} v^{r}\right\|_{0}+h\left\|\zeta\left(\phi_{j}^{-1}\right)^{*} \zeta_{j} \tilde{\Xi}_{\mathscr{G} \mathscr{F}} v^{r}\right\|_{1}+h^{2}\left|\gamma_{1}\left(\left(\phi_{j}^{-1}\right)^{*} \zeta_{j} \tilde{\Xi}_{\mathscr{G} \mathscr{F}} v^{r}\right)\right|_{0} .
\end{aligned}
$$


We notice that the right-hand side of this inequality can be bounded directly by global quantities. First, we have

$$
\left\|\zeta\left(\phi_{j}^{-1}\right)^{*} \zeta_{j} \tilde{\Xi}_{\mathscr{G} \mathscr{F}} v^{r}\right\|_{1} \lesssim\left\|\tilde{\Xi}_{\mathscr{G} \mathscr{F}} v^{r}\right\|_{1} .
$$

Second, we estimate

$$
\left|\gamma_{1}\left(\left(\phi_{j}^{-1}\right)^{*} \zeta_{j} \tilde{\Xi}_{\mathscr{G} \mathscr{F}} v^{r}\right)\right|_{0} \leq\left|\left(D_{x_{n}} \tilde{\Xi}_{\mathscr{G} \mathscr{F}} v^{r}\right)_{\mid x_{n}=0^{+}}\right|_{0},
$$

where

$$
\left(D_{x_{n}} \tilde{\Xi}_{\mathscr{G} \mathscr{F}} v^{r}\right)_{\mid x_{n}=0^{+}}=\left(\tilde{\Xi}_{\mathscr{G} \mathscr{F}} D_{x_{n}} v^{r}\right)_{\mid x_{n}=0^{+}}+(\underbrace{\left[D_{x_{n}}, \tilde{\Xi}_{\mathscr{G} \mathscr{F}}\right]}_{\in h \Psi_{\mathcal{T}}^{0}\left(\mathcal{M}_{+}\right)} v^{r})_{\mid x_{n}=0^{+}} .
$$

Using Proposition B.12 and the trace formula (1.23), we have the estimate

$$
h^{2}\left|\gamma_{1}\left(\left(\phi_{j}^{-1}\right)^{*} \zeta_{j} \tilde{\Xi}_{\mathscr{G} \mathscr{F}} v^{r}\right)\right|_{0} \lesssim h^{2}\left|D_{x_{n}} v_{\mid x_{n}=0^{+}}^{r}\right|_{0}+h^{5 / 2}\left\|v^{r}\right\|_{1} .
$$

Concerning the term with $P_{\varphi, j}^{r}$ on the right-hand side of (4.22), we can proceed as in (4.20)-(4.21) to obtain

$$
\begin{aligned}
\left\|P_{\varphi, j}^{r} \zeta\left(\phi_{j}^{-1}\right)^{*} \zeta_{j} \tilde{\Xi}_{\mathscr{G} \mathscr{F}} v^{r}\right\|_{0} & \\
& =\left\|\left(\phi_{j}^{-1}\right)^{*} P_{\varphi}^{r} \zeta \zeta_{j} \tilde{\Xi}_{\mathscr{G} \mathscr{F}} v^{r}\right\|_{0} \lesssim\left\|P_{\varphi}^{r} \zeta \tilde{\Xi}_{\mathscr{G} \mathscr{F}} v^{r}\right\|_{0}+h\left\|\tilde{\Xi}_{\mathscr{G} \mathscr{F}} v^{r}\right\|_{1} .
\end{aligned}
$$

Moreover, using Proposition B.10, we have $\tilde{\Xi}_{\mathscr{G} \mathscr{F}}\left(1-\Xi_{\mathscr{G} \mathscr{F}}\right) \in h^{\infty} \Psi_{\mathcal{T}}^{-\infty}\left(\mathcal{M}_{+}\right)$, as their local symbols in every chart have disjoint supports by Proposition B.14, because of the supports of $\zeta^{3}$ and $\tilde{\chi} \mathscr{G} \mathscr{F}$. We then obtain, by Proposition B.12,

$h\left\|\tilde{\Xi}_{\mathscr{G} \mathscr{F}} v^{r}\right\|_{1} \lesssim h\left\|\tilde{\boldsymbol{\Xi}}_{\mathscr{G} \mathscr{F}} \boldsymbol{\Xi}_{\mathscr{G} \mathscr{F}} v^{r}\right\|_{1}+h\left\|\tilde{\Xi}_{\mathscr{G} \mathscr{F}}\left(1-\boldsymbol{\Xi}_{\mathscr{G} \mathscr{F}}\right) v^{r}\right\|_{1} \lesssim h\left\|\boldsymbol{\Xi}_{\mathscr{G} \mathscr{F}} v^{r}\right\|_{1}+h^{2}\left\|v^{r}\right\|_{1}$.

We also have

$$
\left\|P_{\varphi}^{r} \zeta \tilde{\Xi}_{\mathscr{G} \mathscr{F}} v^{r}\right\|_{0} \lesssim\left\|\tilde{\boldsymbol{\Xi}}_{\mathscr{G} \mathscr{F}} \zeta P_{\varphi}^{r} v^{r}\right\|_{0}+\left\|\left[P_{\varphi}^{r}, \tilde{\Xi}_{\mathscr{G} \mathscr{F}} \zeta\right] v^{r}\right\|_{0}
$$

Arguing as above with the use of Propositions B.10 and B.14, and also Corollary B.11, we have

$$
\left[P_{\varphi}^{r}, \tilde{\Xi}_{\mathscr{G} \mathscr{F}} \zeta\right]=\underbrace{\left[P_{\varphi}^{r}, \tilde{\Xi}_{\mathscr{G} \mathscr{F}} \zeta\right]}_{\in h \Psi^{1}\left(\mathcal{M}_{+}\right)} \Xi_{\mathscr{G} \mathscr{F}}+\underbrace{\left[P_{\varphi}^{r}, \tilde{\Xi}_{\mathscr{G} \mathscr{F}} \zeta\right]\left(1-\Xi_{\mathscr{G} \mathscr{F}}\right)}_{\in h^{\infty} \Psi^{-\infty}\left(\mathcal{M}_{+}\right)}
$$

so that (4.27) now reads, by Proposition B.12,

$$
\left\|P_{\varphi}^{r} \zeta \tilde{\Xi}_{\mathscr{G} \mathscr{F}} v^{r}\right\|_{0} \lesssim\left\|\zeta P_{\varphi}^{r} v^{r}\right\|_{0}+h\left\|\Xi_{\mathscr{G} \mathscr{F}} v^{r}\right\|_{1}+h^{2}\left\|v^{r}\right\|_{1}
$$

The three estimates (4.25), (4.26) and (4.28) give

$$
\left\|P_{\varphi, j}^{r} \zeta\left(\phi_{j}^{-1}\right)^{*} \zeta_{j} \tilde{\Xi}_{\mathscr{G} \mathscr{F}} v^{r}\right\|_{0} \lesssim\left\|\zeta P_{\varphi}^{r} v^{r}\right\|_{0}+h\left\|\Xi_{\mathscr{G} \mathscr{F}} v^{r}\right\|_{1}+h^{2}\left\|v^{r}\right\|_{1}
$$


Combining (4.22) with (4.23)-(4.24), (4.26) and (4.29), we finally have

$$
\begin{aligned}
& h^{1 / 2}\left|\gamma_{0}\left(\mathrm{Op}_{\mathcal{T}}(\chi \mathscr{G}, j)\left(\phi_{j}^{-1}\right)^{*} \zeta_{j} \tilde{\Xi}_{\mathscr{G} \mathscr{F}} v^{r}\right)\right|_{1}+h^{1 / 2}\left|\gamma_{1}\left(\mathrm{Op}_{\mathcal{T}}\left(\chi_{\mathscr{G}, j}\right)\left(\phi_{j}^{-1}\right)^{*} \zeta_{j} \tilde{\Xi}_{\mathscr{G} \mathscr{F}} v^{r}\right)\right|_{0} \\
& \lesssim\left\|\zeta P_{\varphi}^{r} v^{r}\right\|_{0}+h\left\|\Xi_{\mathscr{G} \mathscr{F}} v^{r}\right\|_{1}+h^{2}\left|D_{x_{n}} v^{r}\right| x_{n}=0^{+}\left.\right|_{0}+h^{2}\left\|v^{r}\right\|_{1} .
\end{aligned}
$$

Then, we need the following lemma to come back to the variable $u_{\mathscr{G}, j}^{r}=$ $\mathrm{Op}_{\mathcal{T}}\left(\chi_{\mathscr{G}, j}\right)\left(\phi_{j}^{-1}\right)^{*} \zeta_{j} v^{r}$ on the left-hand side of (4.30).

Lemma 4.2. There exists $R \in h^{\infty} \Psi_{\mathcal{T}}^{-\infty}\left(\mathcal{M}_{+}\right)$such that

$$
\mathrm{Op}_{\mathcal{T}}\left(\chi \mathcal{G}_{, j}\right)\left(\phi_{j}^{-1}\right)^{*} \zeta_{j} \tilde{\Xi}_{\mathscr{G} \mathscr{F}} v^{r}=u_{\mathscr{G}, j}^{r}+\left(\phi_{j}^{-1}\right)^{*} R v^{r} .
$$

This lemma is proven in Appendix C.6. As a consequence we have

$$
\begin{aligned}
h^{1 / 2}\left|\gamma_{0}\left(u_{\mathscr{G}, j}^{r}\right)\right|_{1} & \lesssim h^{1 / 2}\left|\gamma_{0}\left(\operatorname{Op}_{\mathcal{T}}\left(\chi \chi_{G}, j\right)\left(\phi_{j}^{-1}\right)^{*} \zeta_{j} \tilde{\Xi}_{\mathscr{G} \mathscr{F}} v^{r}\right)\right|_{1}+h^{1 / 2}\left|\gamma_{0}\left(\left(\phi_{j}^{-1}\right)^{*} R v^{r}\right)\right|_{0} \\
& \lesssim h^{1 / 2}\left|\gamma_{0}\left(\operatorname{Op}_{\mathcal{T}}\left(\chi \chi_{\mathscr{G}, j}\right)\left(\phi_{j}^{-1}\right)^{*} \zeta_{j} \tilde{\Xi}_{\mathscr{G} \mathscr{F}} v^{r}\right)\right|_{1}+h^{2}\left\|v^{r}\right\|_{1}
\end{aligned}
$$

by the trace formula (1.23). This, together with estimate (4.30), gives

$$
h^{1 / 2}\left|\gamma_{0}\left(u_{\mathscr{G}, j}^{r}\right)\right|_{1} \lesssim\left\|\zeta P_{\varphi}^{r} v^{r}\right\|_{0}+h\left\|\Xi_{\mathscr{G} \mathscr{F}} v^{r}\right\|_{1}+h^{2}\left\|v^{r}\right\|_{1}+h^{2}\left|D_{x_{n}} v_{\mid x_{n}=0^{+}}^{r}\right|_{0}
$$

Lemma 4.2 also yields

$$
\begin{aligned}
& h^{1 / 2}\left|\gamma_{1}\left(u_{\mathscr{G}, j}^{r}\right)\right|_{0} \lesssim h^{1 / 2}\left|\gamma_{1}\left(\operatorname{Op}_{\mathcal{T}}\left(\chi \mathscr{G}_{, j}\right)\left(\phi_{j}^{-1}\right)^{*} \psi_{j} \tilde{\Xi}_{\mathscr{G} \mathscr{F}} v^{r}\right)\right|_{0}+h^{1 / 2}\left|\gamma_{1}\left(\left(\phi_{j}^{-1}\right)^{*} R v^{r}\right)\right|_{0} \\
& \lesssim h^{1 / 2}\left|\gamma_{1}\left(\operatorname{Op}_{\mathcal{T}}\left(\chi \chi_{\mathscr{G}, j}\right)\left(\phi_{j}^{-1}\right)^{*} \psi_{j} \tilde{\Xi} \mathscr{G}_{\mathscr{F}} v^{r}\right)\right|_{0}+h^{2}\left\|v^{r}\right\|_{1}+h^{2}\left|D_{x_{n}} v_{\mid x_{n}=0^{+}}^{r}\right|_{0} .
\end{aligned}
$$

Combining (4.30) with (4.32), we finally obtain

$$
h^{1 / 2}\left|\gamma_{1}\left(u_{\mathscr{G}, j}^{r}\right)\right|_{0} \lesssim\left\|\zeta P_{\varphi}^{r} v^{r}\right\|_{0}+h\left\|\Xi_{\mathscr{G} \mathscr{F}} v^{r}\right\|_{1}+h^{2}\left\|v^{r}\right\|_{1}+h^{2}\left|D_{x_{n}} v_{\mid x_{n}=0^{+}}^{r}\right|_{0} .
$$

On the "l" side, we apply the Carleman method. By the properties of the weight function of Section 3.1 and in particular by (3.18), and by Lemma 2 in [LR95], we then have

$$
\begin{array}{r}
h\left\|u_{\mathscr{G}, j}^{l}\right\|_{1}^{2}+\operatorname{Re}\left(h \mathscr{B}^{l}\left(u_{\mathscr{G}, j}^{l}\right)+h^{2}\left(\left(D_{n} u_{\mathscr{G}, j}^{l}+L_{1}^{l} u_{\mathscr{G}, j}^{l}\right)_{\mid x_{n}=0^{+}}, L_{0^{\prime}}^{l} u_{\mathscr{G}_{, j}}^{l}{ }_{\mid x_{n}=0^{+}}\right)_{0}\right) \\
\lesssim\left\|P_{\varphi, j} u_{\mathscr{G}, j}^{l}\right\|_{0}^{2},
\end{array}
$$

for $0<h \leq h_{0}, h_{0}$ sufficiently small, where $L_{1}^{l} \in \mathscr{D}_{\mathcal{T}}^{1}$ and $L_{0}^{l} \in \Psi_{\mathcal{T}}^{0}$. The quadratic form $\mathscr{B}^{l}$ is given by

$$
\begin{aligned}
& \mathscr{B}^{l}(\psi) \\
& =\left(\left(\begin{array}{cc}
2 \partial_{x_{n}} \varphi_{j \mid x_{n}=0^{+}}^{l} & B_{1}^{l} \\
B_{1}^{l \prime} & B_{2}^{l}
\end{array}\right)\left(\begin{array}{l}
\gamma_{1}(\psi) \\
\gamma_{0}(\psi)
\end{array}\right),\left(\begin{array}{l}
\gamma_{1}(\psi) \\
\gamma_{0}(\psi)
\end{array}\right)\right)_{0}, \operatorname{supp}(\psi) \subset\left(0, X_{0}\right) \times \tilde{U}_{j} \times[0,2 \varepsilon),
\end{aligned}
$$

where $B_{1}^{l}, B_{1}^{l \prime} \in \mathscr{D}_{\mathcal{T}}^{1}$ with principal symbols $\sigma\left(B_{1}^{l}\right)=\sigma\left(B_{1}^{l \prime}\right)=2\left(\phi_{j}^{-1}\right)^{*} q_{1 \mid x_{n}=0^{+}}^{l}$, and $B_{2}^{l} \in \mathscr{D}_{\mathcal{T}}^{2}$ with $\sigma\left(B_{2}^{l}\right)=-2 \partial_{x_{n}} \varphi_{j}^{l}\left(\phi_{j}^{-1}\right)^{*} q_{2 \mid x_{n}=0^{+}}^{l}$. 
Observe that

$$
\begin{aligned}
&\left|\left(\left(D_{n} u_{\mathscr{G}, j}^{l}+L_{1}^{l} u_{\mathscr{G}, j}^{l}\right)_{\mid x_{n}=0^{+}},\left.L_{0}^{l} u_{\mathscr{G}, j}^{l}\right|_{\mid x_{n}=0^{+}}\right)_{0}\right| \lesssim\left|\gamma_{1}\left(u_{\mathscr{G}_{, j}}^{l}\right)\right|_{0}^{2}+\left|\gamma_{0}\left(u_{\mathscr{G}_{, j}}^{l}\right)\right|_{1}^{2}, \\
&\left|\mathscr{B}^{l}\left(u_{\mathscr{G}, j}^{l}\right)\right| \lesssim\left|\gamma_{0}\left(u_{\mathscr{G}_{, j}}^{l}\right)\right|_{1}^{2}+\left|\gamma_{1}\left(u_{\mathscr{G}_{, j}}^{l}\right)\right|_{0}^{2} .
\end{aligned}
$$

Now, using (4.34), together with the estimates (4.36) and (4.37), we have

$$
h\left\|u_{\mathscr{G}, j}^{l}\right\|_{1}^{2} \lesssim\left\|P_{\varphi, j}^{l} u_{\mathscr{G}, j}^{l}\right\|_{0}^{2}+h\left|\gamma_{0}\left(u_{\mathscr{G}, j}^{l}\right)\right|_{1}^{2}+h\left|\gamma_{1}\left(u_{\mathscr{G}, j}^{l}\right)\right|_{0}^{2} .
$$

It remains to estimate the traces on the "l" side by the traces on the " $r$ " side, through the transmission conditions $\left(\mathrm{TC}_{\bullet}, j\right)$ :

$$
\left\{\begin{array}{l}
\gamma_{0}\left(u_{\mathscr{G}, j}^{l}\right)=\gamma_{0}\left(u_{\mathscr{G}, j}^{r}\right)+\theta_{\mathscr{G}, j}^{l}-\theta_{\mathscr{G}, j}^{r}, \\
\gamma_{1}\left(u_{\mathscr{G}, j}^{l}\right)=\frac{\delta c_{j}^{s}}{h i c_{j}^{l}} P_{\varphi, j}^{s}\left(\gamma_{0}\left(u_{\mathscr{G}, j}^{r}\right)-\theta_{\mathscr{G}, j}^{r}\right)-\beta \gamma_{1}\left(u_{\mathscr{G}, j}^{r}\right)+k \gamma_{0}\left(u_{\mathscr{G}, j}^{r}\right)-\tilde{G}_{1}, \\
u_{\mathscr{G}, j}^{s}=\gamma_{0}\left(u_{\mathscr{G}, j}^{r}\right)-\theta_{\mathscr{G}, j}^{r} .
\end{array}\right.
$$

As a consequence, $\gamma_{0}\left(u_{\mathscr{G}, j}^{l}\right)$ and $\gamma_{1}\left(u_{\mathscr{G}, j}^{l}\right)$ can be estimated as follows:

$$
\left\{\begin{array}{l}
\left|\gamma_{0}\left(u_{\mathscr{G}, j}^{l}\right)\right|_{1} \leq\left|\gamma_{0}\left(u_{\mathscr{G}, j}^{r}\right)\right|_{1}+\left|\theta_{\mathscr{G}, j}^{l}\right|_{1}+\left|\theta_{\mathscr{G}, j}^{r}\right|_{1}, \\
\left|\gamma_{1}\left(u_{\mathscr{G}, j}^{l}\right)\right|_{0} \lesssim\left|\gamma_{1}\left(u_{\mathscr{G}, j}^{r}\right)\right|_{0}+\frac{\delta}{h}\left|P_{\varphi, j}^{s} \gamma_{0}\left(u_{\mathscr{G}, j}^{r}\right)\right|_{0}+\frac{\delta}{h}\left|P_{\varphi, j}^{s} \theta_{\mathscr{G}, j}^{r}\right|_{0}+\left|\gamma_{0}\left(u_{\mathscr{G}, j}^{r}\right)\right|_{0}+\left|\tilde{G}_{1}\right|_{0} .
\end{array}\right.
$$

We now prove that, on the support of $\chi_{\mathscr{G}, j}$, the operator $P_{\varphi, j}^{s}$ is of order 0. For this, let $\tilde{\chi} \in \mathscr{C}_{c}^{\infty}\left(T^{*}\left(\mathbb{R}^{n}\right)\right)$ be equal to 1 on a neighborhood of $\operatorname{supp}\left(\left.\chi \chi_{\mathscr{G}, j}\right|_{x_{n}=0^{+}}\right)$. Then

$$
\begin{aligned}
\gamma_{0}\left(u_{\mathscr{G}, j}^{r}\right) & =\mathrm{Op}_{\mathcal{T}}\left(\chi_{\mathscr{G}, j}\right) v_{j \mid x_{n}=0^{+}}^{r} \\
& =\mathrm{Op}_{\mathcal{T}}(\tilde{\chi}) \mathrm{Op}_{\mathcal{T}}\left(\chi_{\mathscr{G}, j}\right) v_{j \mid x_{n}=0^{+}}^{r}+\underbrace{\mathrm{Op}_{\mathcal{T}}(1-\tilde{\chi}) \mathrm{Op}_{\mathcal{T}}\left(\chi_{\mathscr{G}, j}\right)}_{\in h^{\infty} \Psi_{\mathcal{T}}^{-\infty}} v_{\left.\right|_{\mid x_{n}=0^{+}} ^{r},},
\end{aligned}
$$

which yields

$$
P_{\varphi, j}^{s} \gamma_{0}\left(u_{\mathscr{G}, j}^{r}\right)=\underbrace{\left(P_{\varphi, j}^{s} \mathrm{Op}_{\mathcal{T}}(\tilde{\chi})\right)}_{\in \Psi_{\mathcal{T}}^{0}} \gamma_{0}\left(u_{\mathscr{G}, j}^{r}\right)+\underbrace{P_{\varphi, j}^{s} \mathrm{Op}_{\mathcal{T}}(1-\tilde{\chi}) \mathrm{Op}_{\mathcal{T}}\left(\chi_{\mathscr{G}, j}\right)}_{\in h^{\infty} \Psi_{\mathcal{T}}^{-\infty}} v_{j}^{r}{\mid x_{n}=0^{+}} .
$$

This, together with the trace formula (1.23), gives the estimate

$$
\frac{\delta}{h}\left|P_{\varphi, j}^{s} \gamma_{0}\left(u_{\mathscr{G}, j}^{r}\right)\right|_{0} \leq C \frac{\delta}{h}\left|\gamma_{0}\left(u_{\mathscr{G}, j}^{r}\right)\right|_{0}+C_{N} \delta h^{N}\left\|v_{j}^{r}\right\|_{1}, \quad N \in \mathbb{N} .
$$

Similarly, we have the estimate

$$
\frac{\delta}{h}\left|P_{\varphi, j}^{s} \theta_{\mathscr{G}, j}^{r}\right|_{0} \leq C \frac{\delta}{h}\left|\theta_{\mathscr{G}, j}^{r}\right|_{0}+C_{N} \delta h^{N}\left|\theta_{\varphi, j}^{r}\right|_{0} \lesssim \frac{\delta}{h}\left|\theta_{\varphi, j}^{r}\right|_{0} .
$$


The last two estimates and the second estimate of (4.39) yield

$$
\begin{aligned}
\left|\gamma_{1}\left(u_{\mathscr{G}, j}^{l}\right)\right|_{0} \lesssim & \left|\gamma_{1}\left(u_{\mathscr{G}, j}^{r}\right)\right|_{0}+\left(1+\frac{\delta}{h}\right)\left|\gamma_{0}\left(u_{\mathscr{G}, j}^{r}\right)\right|_{0} \\
& +\frac{\delta}{h}\left|\theta_{\varphi, j}^{r}\right|_{0}+\left|\tilde{G}_{1}\right|_{0}+C_{N} \delta h^{N}\left\|v_{j}^{r}\right\|_{1}, \quad N \in \mathbb{N} .
\end{aligned}
$$

Using estimates (4.31) and (4.33) to bound the traces on the " $r$ " side, we obtain

$$
\begin{aligned}
h^{1 / 2}\left|\gamma_{1}\left(u_{\mathscr{G}, j}^{l}\right)\right|_{0} \lesssim & \left(1+\frac{\delta}{h}\right)\left(\left\|\zeta P_{\varphi}^{r} v^{r}\right\|_{0}+h\left\|\Xi_{\mathscr{G} \mathscr{F}} v^{r}\right\|_{1}+h^{2}\left\|v^{r}\right\|_{1}+h^{2}\left|D_{x_{n}} v_{\mid x_{n}=0^{+}}^{r}\right|_{0}\right) \\
& +\frac{\delta}{h^{1 / 2}}\left|\theta_{\varphi, j}^{r}\right|_{0}+h^{1 / 2}\left|\tilde{G}_{1}\right|_{0},
\end{aligned}
$$

for $0<h \leq h_{0}$, and using (4.11) to estimate the remainder, we have

$$
\begin{aligned}
h^{1 / 2}\left|\gamma_{1}\left(u_{\mathscr{G}, j}^{l}\right)\right|_{0} \lesssim & \left(1+\frac{\delta}{h}\right)\left(\left\|\zeta P_{\varphi}^{r} v^{r}\right\|_{0}+h\left\|\Xi \mathscr{G} \mathscr{F} v^{r}\right\|_{1}+h^{2}\left\|v^{r}\right\|_{1}+h^{2}\left|D_{x_{n}} v_{\mid x_{n}=0^{+}}^{r}\right|_{0}\right. \\
& \left.+h^{3 / 2}\left|v_{j}^{s}\right|_{1}+h^{1 / 2}\left|\theta_{\varphi, j}^{r}\right|_{0}\right)+h^{1 / 2}\left|\Theta_{\varphi, j}^{s}\right|_{0}+h^{1 / 2}\left|\theta_{\varphi, j}^{l}\right|_{0},
\end{aligned}
$$

We now observe that the first line of (4.39) together with (4.31) yields

$$
\begin{aligned}
h^{1 / 2}\left|\gamma_{0}\left(u_{\mathscr{G}, j}^{l}\right)\right|_{1} \lesssim & \left\|\zeta P_{\varphi}^{r} v^{r}\right\|_{0}+h\left\|\Xi \mathscr{G F} v^{r}\right\|_{1}+h^{2}\left\|v^{r}\right\|_{1}+h^{2}\left|D_{x_{n}} v_{\mid x_{n}=0^{+}}^{r}\right|_{0} \\
& +h^{1 / 2}\left|\theta_{\varphi, j}^{l}\right|_{1}+h^{1 / 2}\left|\theta_{\varphi, j}^{r}\right|_{1} .
\end{aligned}
$$

Combining (4.5) with (4.38), (4.40) and (4.41) we obtain

$$
\begin{aligned}
& h\left\|u_{\mathscr{G}, j}^{l}\right\|_{1}^{2}+h\left|\gamma_{0}\left(u_{\mathscr{G}, j}^{l}\right)\right|_{1}^{2}+h\left|\gamma_{1}\left(u_{\mathscr{G}, j}^{l}\right)\right|_{0}^{2} \\
& \quad \lesssim\left(1+\frac{\delta^{2}}{h^{2}}\right)\left(\left\|\zeta P_{\varphi}^{r} v^{r}\right\|_{0}^{2}+h^{2}\left\|\Xi \mathscr{G} \mathscr{F} v^{r}\right\|_{1}^{2}+h^{4}\left\|v^{r}\right\|_{1}^{2}+h^{4}\left|D_{x_{n}} v_{\mid x_{n}=0^{+}}^{r}\right|_{0}^{2}+h^{3}\left|v_{j}^{s}\right|_{1}^{2}\right) \\
& \quad+h\left|\theta_{\varphi, j}^{l}\right|_{1}^{2}+\frac{\delta^{2}}{h}\left|\theta_{\varphi, j}^{r}\right|_{0}^{2}+h\left|\theta_{\varphi, j}^{r}\right|_{1}^{2}+h\left|\Theta_{\varphi, j}^{s}\right|_{0}^{2}+\left\|P_{\varphi, j}^{l} v_{j}^{l}\right\|_{0}^{2}+h^{2}\left\|v_{j}^{l}\right\|_{1}^{2} .
\end{aligned}
$$

This is a local version of (4.14). Patching together on $\mathcal{M}_{+}$the local Carleman estimates (4.42) as we did in (4.16)-(4.21) yields (4.14). This concludes the proof of Proposition 4.1 .

\subsection{Estimate in the region $\mathscr{F}$}

Here, we prove a Carleman estimate for $u_{\mathscr{F}, j}$, and consequently for $\Xi_{\mathscr{F}} v$. Making use of the Calderón projector technique for both $P_{\varphi, j}^{r}$ and $P_{\varphi, j}^{l}$, we obtain the following partial estimate. 
Proposition 4.3. Suppose that the weight function $\varphi$ has the properties listed in Section 3.1. Then, for all $\delta_{0}>0$, there exist $C>0$ and $h_{0}>0$ such that, for all $0<\delta \leq \delta_{0}$ and $0<h \leq h_{0}$, and for $v^{r / l} \in \mathscr{C}_{c}^{\infty}\left(\left(0, X_{0}\right) \times S \times[0,2 \varepsilon)\right)$ and $v^{s} \in \mathscr{C}_{c}^{\infty}\left(\left(0, X_{0}\right) \times S\right)$ satisfying (3.9), we have

$$
\begin{aligned}
\left\|\Xi_{\mathscr{F}} v^{r}\right\|_{1}^{2}+h\left|\Xi_{\mathscr{F}} v_{\mid x_{n}=0^{+}}^{r}\right|_{1}^{2} & +h\left|D_{x_{n}} \Xi_{\mathscr{F}} v_{\mid x_{n}=0^{+}}^{r}\right|_{0}^{2} \\
& \leq C\left(\left\|P_{\varphi}^{r} v^{r}\right\|_{0}^{2}+h^{2}\left\|v^{r}\right\|_{1}^{2}+h^{4}\left|D_{x_{n}} v_{\mid x_{n}=0^{+}}^{r}\right|_{0}^{2}\right),
\end{aligned}
$$

and

$$
\begin{aligned}
&\left\|\Xi_{\mathscr{F}} v^{l}\right\|_{1}^{2}+h\left|\Xi_{\mathscr{F}} v_{\mid x_{n}=0^{+}}^{l}\right|_{1}^{2}+h\left|D_{x_{n}} \Xi_{\mathscr{F}} v_{\mid x_{n}=0^{+}}^{l}\right|_{0}^{2} \\
& \leq C\left(\left\|P_{\varphi}^{l} v^{l}\right\|_{0}^{2}+h^{2}\left\|v^{l}\right\|_{1}^{2}+h^{4}\left|D_{x_{n}} v_{\mid x_{n}=0^{+}}^{l}\right|_{0}^{2}+\left\|P_{\varphi}^{r} v^{r}\right\|_{0}^{2}\right. \\
&\left.+h^{2}\left\|v^{r}\right\|_{1}^{2}+h^{4}\left|D_{x_{n}} v_{\mid x_{n}=0^{+}}^{r}\right|_{0}^{2}+h\left|\theta_{\varphi}^{l}\right|_{1}^{2}+h\left|\theta_{\varphi}^{r}\right|_{1}^{2}\right) .
\end{aligned}
$$

Proof. Here, the functions $u_{\mathscr{F}, j}^{r / l}, j \in J$ satisfy $\left(\mathrm{TC}_{\bullet, j}\right)$, with $\bullet=\mathscr{F}$. On both the " $r$ " and " $l$ " sides, the root configuration described in Lemma 3.6 (and represented in Figure 5)
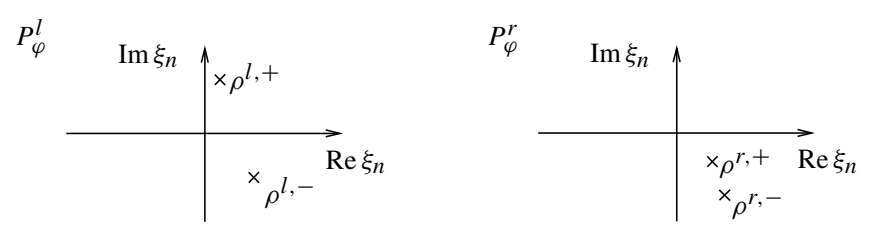

Fig. 5. Root configuration in the region $\mathscr{F}$.

allows us to use the Calderón projector technique used in [LR97, LR10]. According to [LR10, Remark 2.5] and using (2.59)-(2.61) therein, applied with $v^{d}$ replaced here by $v_{j}^{r}$, we have

$$
\begin{aligned}
\left\|u_{\mathscr{F}, j}^{r}\right\|_{1}+h^{1 / 2}\left|\gamma_{0}\left(u_{\mathscr{F}, j}^{r}\right)\right|_{1}+ & h^{1 / 2}\left|\gamma_{1}\left(u_{\mathscr{F}, j}^{r}\right)\right|_{0} \\
& \lesssim\left\|P_{\varphi, j}^{r} v_{j}^{r}\right\|_{0}+h\left\|v_{j}^{r}\right\|_{1}+h^{2}\left|D_{x_{n}} v_{j}^{r}\right|_{\mid x_{n}=0^{+}} \mid 0 .
\end{aligned}
$$

This is a local version of (4.43). Patching together on $\mathcal{M}_{+}$the local Carleman estimates (4.45) as we did in (4.16)-(4.21) yields (4.43).

On the "l" side, since both roots are separated by the real axis (see Figure 5) we only obtain one relation between the two traces at the interface: according to [LR10, Eq. (2.67)], we have

$$
\begin{aligned}
\left\|u_{\mathscr{F}, j}^{l}\right\|_{1} \lesssim & \left\|P_{\varphi, j}^{l} v_{j}^{l}\right\|_{0}+h\left\|v_{j}^{l}\right\|_{1}+h^{1 / 2}\left|\gamma_{0}\left(u_{\mathscr{F}, j}^{l}\right)\right|_{1}+h^{1 / 2}\left|\gamma_{1}\left(u_{\mathscr{F}, j}^{l}\right)\right|_{0} \\
& +h^{2}\left|D_{x_{n}} v_{j_{X_{n}=0^{+}}}^{l}\right|_{0},
\end{aligned}
$$

together with the following relation between the two traces [LR10, Eq. (2.68)]:

$$
\left(1-\mathrm{Op}_{\mathcal{T}}\left(a^{l}\right)\right) \gamma_{0}\left(u_{\mathscr{F}, j}^{l}\right)=\mathrm{Op}_{\mathcal{T}}\left(b^{l}\right) \gamma_{1}\left(u_{\mathscr{F}, j}^{l}\right)+G_{2}^{l},
$$


where $a^{l} \in S_{\mathcal{T}}^{0}$ and $b^{l} \in S_{\mathcal{T}}^{-1}$ have principal parts respectively

$$
a_{0}^{l}=-\left.\left(\tilde{\chi} \frac{\rho_{j}^{l,-}}{\rho_{j}^{l,+}-\rho_{j}^{l,-}}\right)\right|_{x_{n}=0^{+}} \quad \text { and } \quad b_{-1}^{l}=\left.\left(\tilde{\chi} \frac{1}{\rho_{j}^{l,+}-\rho_{j}^{l,-}}\right)\right|_{x_{n}=0^{+}},
$$

where $\rho_{j}^{l, \pm}$ are the roots of $p_{\varphi, j}^{l}$ (i.e. $\rho_{j}^{l, \pm}=\left(\phi_{j}^{-1}\right)^{*} \rho^{l, \pm}$ with $\rho^{l, \pm}$ described in Lemma 3.6) and $\tilde{\chi} \in \mathscr{C}_{c}^{\infty}\left(T^{*}\left(\mathbb{R}^{n}\right)\right)$ is compactly supported and equal to 1 on a neighborhood of the support of $\chi_{\mathscr{F}, j} j_{\mid x_{n}=0^{+}}$. The remainder $G_{2}^{l}$ (coming from the Calderón projector method) satisfies [LR10, Eq. (2.69)]

$$
\left|G_{2}^{l}\right|_{1} \lesssim h^{-1 / 2}\left(\left\|P_{\varphi, j}^{l} v_{j}^{l}\right\|_{0}+h\left\|v_{j}^{l}\right\|_{1}+h^{2}\left|D_{x_{n}} v_{j_{\mid x_{n}=0^{+}}^{l}}\right| 0\right) .
$$

Let $\hat{\chi} \in \mathscr{C}^{\infty}\left(T^{*}\left(\mathbb{R}^{n}\right)\right)$ satisfy the same requirements as $\tilde{\chi}$ with $\tilde{\chi}$ equal to 1 in a neighborhood of the support of $\hat{\chi}$. Since $b_{-1}^{l}$ does not vanish in a neighborhood of $\operatorname{supp}(\hat{\chi})$, one can introduce a parametrix for $\mathrm{Op}_{\mathcal{T}}\left(b^{l}\right)$, say $\mathrm{Op}_{\mathcal{T}}(e)$, with $e \in S_{\mathcal{T}}^{1}$, satisfying

$$
\mathrm{Op}_{\mathcal{T}}(e) \mathrm{Op}_{\mathcal{T}}\left(b^{l}\right)=\mathrm{Op}_{\mathcal{T}}(\hat{\chi})+R, \quad R \in h^{\infty} \Psi_{\mathcal{T}}^{-\infty} .
$$

Applying this parametrix to (4.47) gives the estimate

$\left|\gamma_{1}\left(u_{\mathscr{F}, j}^{l}\right)\right|_{0} \lesssim\left|\gamma_{0}\left(u_{\mathscr{F}, j}^{l}\right)\right|_{1}+\left|G_{2}^{l}\right|_{1}+C_{N} h^{N}\left(\left\|v_{j}^{l}\right\|_{1}+\left|D_{x_{n}} v_{j \mid x_{n}=0^{+}}^{l}\right|_{0}\right), \quad N \in \mathbb{N}$.

Here, we have used the trace formula (1.23) together with

$$
\begin{aligned}
& \gamma_{1}\left(u_{\mathscr{F}, j}^{l}\right)=\mathrm{Op}_{\mathcal{T}}(\hat{\chi}) \gamma_{1}\left(u_{\mathscr{F}, j}^{l}\right)+\underbrace{\left(\left(1-\mathrm{Op}_{\mathcal{T}}(\hat{\chi})\right) \mathrm{Op}_{\mathcal{T}}\left(\chi_{\mathscr{F}, j}\right)_{\mid x_{n}=0^{+}}\right)}_{\in h^{\infty} \Psi_{\mathcal{T}}^{-\infty}} D_{x_{n}} v_{j_{\mid x_{n}=0^{+}}^{l}} \\
& +\underbrace{\left(\left(1-\mathrm{Op}_{\mathcal{T}}(\hat{\chi})\right)\left[D_{x_{n}}, \mathrm{Op}_{\mathcal{T}}\left(\chi_{\mathscr{F}, j}\right)\right]_{\mid x_{n}=0^{+}}\right)}_{\in h^{\infty} \Psi_{\mathcal{T}}^{-\infty}} v_{j_{\mid x_{n}=0^{+}}}^{l} .
\end{aligned}
$$

We now use the second equation in the transmission conditions $\left(\mathrm{TC}_{\bullet}, j\right)$, which by (4.45), yields

$$
\begin{aligned}
h^{1 / 2}\left|\gamma_{0}\left(u_{\mathscr{F}, j}^{l}\right)\right|_{1} & \leq h^{1 / 2}\left|\gamma_{0}\left(u_{\mathscr{F}, j}^{r}\right)\right|_{1}+h^{1 / 2}\left|\theta_{\mathscr{F}, j}^{l}\right|_{1}+h^{1 / 2}\left|\theta_{\mathscr{F}, j}^{r}\right|_{1} \\
& \lesssim\left\|P_{\varphi, j}^{r} v_{j}^{r}\right\|_{0}+h\left\|v_{j}^{r}\right\|_{1}+h^{2}\left|D_{x_{n}} v_{\left.\right|_{\mid x_{n}=0^{+}} ^{r}}^{r}\right|_{0}+h^{1 / 2}\left|\theta_{\mathscr{F}, j}^{l}\right|_{1}+h^{1 / 2}\left|\theta_{\mathscr{F}, j}^{r}\right|_{1} .
\end{aligned}
$$

This estimate together with (4.48) and (4.49) provides an estimate for $\left|\gamma_{1}\left(u_{\mathscr{F}, j}^{l}\right)\right|_{0}$, which, summed with (4.46), yields

$$
\begin{aligned}
\left\|u_{\mathscr{F}, j}^{l}\right\|_{1}+h^{1 / 2}\left|\gamma_{0}\left(u_{\mathscr{F}, j}^{l}\right)\right|_{1}+h^{1 / 2}\left|\gamma_{1}\left(u_{\mathscr{F}, j}^{l}\right)\right|_{0} \lesssim\left\|P_{\varphi, j}^{l} v_{j}^{l}\right\|_{0}+h\left\|v_{j}^{l}\right\|_{1}+h^{2}\left|D_{x_{n}} v_{j \mid x_{n}=0^{+}}^{l}\right|_{0} \\
+\left\|P_{\varphi, j}^{r} v_{j}^{r}\right\|_{0}+h\left\|v_{j}^{r}\right\|_{1}+h^{2}\left|D_{x_{n}} v_{j \mid x_{n}=0^{+}}^{r}\right|_{0}+h^{1 / 2}\left|\theta_{\mathscr{F}, j}^{l}\right|_{1}+h^{1 / 2}\left|\theta_{\mathscr{F}, j}^{r}\right|_{1} .
\end{aligned}
$$

This is a local version of (4.44). Patching together on $\mathcal{M}_{+}$such local estimates as we did in (4.16)-(4.21) yields (4.44). This concludes the proof of Proposition 4.3. 


\subsection{Estimate in the region $\mathscr{Z}$}

Here, we prove a Carleman estimate for $u \mathscr{Z}, j$, and consequently for $\Xi_{\mathscr{Z}} v$.

As a consequence of property (3.6) of the weight function (see also (3.16)) and the compactness of $\left[0, X_{0}\right] \times S \times[0,2 \varepsilon]$, we remark that in the region $\mathscr{Z}$, there exists $K_{1}>0$ such that

$$
\left(\partial_{x_{n}} \varphi^{r}\right)^{2}-\mu^{r} \geq \min \left(\partial_{x_{n}} \varphi^{r}\right)^{2}-2 \epsilon_{1} \geq K_{1}>0
$$

for $\epsilon_{1}$ sufficiently small (the constant $\epsilon_{1}$ is used in the definition of the microlocal regions in (3.22)).

Making use of the Calderón projector technique for $P_{\varphi, j}^{l}$, and standard techniques to prove Carleman estimates for $P_{\varphi, j}^{r}$, we obtain the following partial estimate.

Proposition 4.4. Suppose that the weight function $\varphi$ has the properties listed in Section 3.1. Then, for all $\delta_{0}>0$, there exist $C>0$ and $h_{0}>0$ such that, for all $0<\delta \leq \delta_{0}$ and $0<h \leq h_{0}$, and for $v^{r / l} \in \mathscr{C}_{c}^{\infty}\left(\left(0, X_{0}\right) \times S \times[0,2 \varepsilon)\right)$ and $v^{s} \in \mathscr{C}_{c}^{\infty}\left(\left(0, X_{0}\right) \times S\right)$ satisfying (3.9), we have

$$
\begin{aligned}
h \| & \Xi_{\mathscr{Z}} v^{r} \|_{1}^{2}+h\left(1+\frac{\delta^{2}}{h^{2}}\right)\left|\Xi_{\mathscr{Z}} v_{\mid x_{n}=0^{+}}^{r}\right|_{1}^{2}+h\left|D_{x_{n}} \Xi_{\mathscr{Z}} v_{\mid x_{n}=0^{+}}^{r}\right|_{0}^{2} \\
\leq & C\left(\left\|P_{\varphi}^{r} v^{r}\right\|_{0}^{2}+h^{2}\left\|v^{r}\right\|_{1}^{2}+h\left(\delta^{2}+h^{2}\right)\left|v^{s}\right|_{1}^{2}+\left\|P_{\varphi}^{l} v^{l}\right\|_{0}^{2}+h^{2}\left\|v^{l}\right\|_{1}^{2}+h^{4}\left|D_{x_{n}} v_{\mid x_{n}=0^{+}}^{l}\right|_{0}^{2}\right. \\
& \left.+\frac{\delta^{2}}{h}\left|\theta_{\varphi}^{r}\right|_{0}^{2}+h\left|\theta_{\varphi}^{l}\right|_{1}^{2}+h\left|\theta_{\varphi}^{r}\right|_{1}^{2}+h\left|\Theta_{\varphi}^{s}\right|_{0}^{2}\right)
\end{aligned}
$$

and

$$
\begin{aligned}
& \left\|\Xi_{\mathscr{Z}} v^{l}\right\|_{1}^{2}+h\left|\Xi_{\mathscr{Z}} v_{\mid x_{n}=0^{+}}^{l}\right|_{1}^{2}+h\left|D_{x_{n}} \Xi_{\mathscr{Z}} v_{\mid x_{n}=0^{+}}^{l}\right|_{0}^{2} \\
& \leq C\left(\left\|P_{\varphi}^{l} v^{l}\right\|_{0}^{2}+h^{2}\left\|v^{l}\right\|_{1}^{2}+h^{4}\left|D_{x_{n}} v_{\mid x_{n}=0^{+}}^{l}\right|_{0}^{2}+h^{3}\left|v^{s}\right|_{1}^{2}+\frac{h^{2}}{\delta^{2}+h^{2}}\left(\left\|P_{\varphi}^{r} v^{r}\right\|_{0}^{2}+h^{2}\left\|v^{r}\right\|_{1}^{2}\right)\right. \\
& \left.\quad+h\left|\theta_{\varphi}^{l}\right|_{1}^{2}+h\left|\theta_{\varphi}^{r}\right|_{1}^{2}+\frac{h^{3}}{\delta^{2}+h^{2}}\left|\Theta_{\varphi}^{s}\right|_{0}^{2}\right) .
\end{aligned}
$$

Proof. The function $u_{\mathscr{Z}, j}$ satisfies $\left(\mathrm{TC}_{\bullet}, j\right.$ ), with $\bullet=\mathscr{Z}$. On the " $l$ " side, the root configuration described in Lemma 3.6 (and represented in Figure 3b) allows us to apply the Calderón projector technique as in [LR97, LR10]. Since the two roots are separated by the real axis we only obtain one relation between the two traces at the interface: according to [LR10, Eq. (2.67)], we have

$$
\begin{aligned}
\left\|u_{\mathscr{Z}, j}^{l}\right\|_{1} \lesssim & \left\|P_{\varphi, j}^{l} v_{j}^{l}\right\|_{0}+h\left\|v_{j}^{l}\right\|_{1}+h^{1 / 2}\left|\gamma_{0}\left(u_{\mathscr{Z}, j}^{l}\right)\right|_{1}+h^{1 / 2}\left|\gamma_{1}\left(u_{\mathscr{Z}, j}^{l}\right)\right|_{0} \\
& +h^{2}\left|D_{x_{n}} v_{j \mid x_{n}=0^{+}}^{l}\right|_{0}
\end{aligned}
$$

together with the following relation between the two traces [LR10, Eq. (2.68)]:

$$
\left(1-\mathrm{Op}_{\mathcal{T}}\left(a^{l}\right)\right) \gamma_{0}\left(u_{\mathscr{Z}, j}^{l}\right)=\mathrm{Op}_{\mathcal{T}}\left(b^{l}\right) \gamma_{1}\left(u_{\mathscr{Z}, j}^{l}\right)+G_{2}^{l},
$$


where $a^{l} \in S_{\mathcal{T}}^{0}$ and $b^{l} \in S_{\mathcal{T}}^{-1}$ have principal parts respectively

$$
a_{0}^{l}=-\left.\left(\tilde{\chi} \frac{\rho_{j}^{l,-}}{\rho_{j}^{l,+}-\rho_{j}^{l,-}}\right)\right|_{x_{n}=0^{+}} \quad \text { and } \quad b_{-1}^{l}=\left.\left(\tilde{\chi} \frac{1}{\rho_{j}^{l,+}-\rho_{j}^{l,-}}\right)\right|_{x_{n}=0^{+}},
$$

where $\rho_{j}^{l, \pm}$ are the roots of $p_{\varphi, j}^{l}$ (i.e. $\rho_{j}^{l, \pm}=\left(\phi_{j}^{-1}\right)^{*} \rho^{l, \pm}$ with $\rho^{l, \pm}$ described in Lemma 3.6) and $\tilde{\chi} \in \mathscr{C}_{c}^{\infty}\left(T^{*}\left(\mathbb{R}^{n}\right)\right)$ is equal to 1 on a neighborhood of the support of $\left.\chi_{\mathscr{Z}, j}\right|_{\mid x_{n}=0^{+}}$and equal to zero in a neighborhood of

$$
\left(\left(\phi_{j}^{-1}\right)^{*} \mathscr{G}\right) \cap\left\{x_{n}=0\right\}=\left\{\left(x_{0}, \phi_{j}(y) ; \xi_{0},{ }^{t} d \phi_{j}^{-1}\left(\phi_{j}(y)\right) \eta\right) ;\left(x_{0}, y, 0 ; \xi_{0}, \eta\right) \in \mathscr{G}\right\} .
$$

The remainder $G_{2}^{l}$ (coming from the Calderón projector method) satisfies [LR10, Eq. (2.69)]:

$$
\left|G_{2}^{l}\right|_{1} \lesssim h^{-1 / 2}\left(\left\|P_{\varphi, j}^{l} v_{j}^{l}\right\|_{0}+h\left\|v_{j}^{l}\right\|_{1}+h^{2}\left|D_{x_{n}} v_{j_{\mid x_{n}=0^{+}}}^{l}\right| 0\right) .
$$

On the " $r$ " side, we apply the Carleman method to the operators $P_{\varphi, j}^{r}$. By the properties of the weight function of Section 3.1, and in particular by (3.18), and by Lemma 2 in [LR95], we then have

$$
\begin{aligned}
h\left\|u_{\mathscr{Z}, j}^{r}\right\|_{1}^{2}+\operatorname{Re}\left(h \mathscr{B}^{r}\left(u_{\mathscr{Z}, j}^{r}\right)+h^{2}\left(\left(D_{n} u_{\mathscr{Z}, j}^{r}+L_{1}^{r} u_{\mathscr{Z}, j}^{r}\right)_{\mid x_{n}=0^{+}},\left.L_{0}^{r} u_{\mathscr{Z}, j}^{r}\right|_{\mid x_{n}=0^{+}}\right)_{0}\right) \\
\lesssim\left\|P_{\varphi, j} u_{\mathscr{Z}, j}^{r}\right\|_{0}^{2}
\end{aligned}
$$

for $h$ sufficiently small, where $L_{1}^{r} \in \mathscr{D}_{\mathcal{T}}^{1}, L_{0}^{r} \in \Psi_{\mathcal{T}}^{0}$. The quadratic form $\mathscr{B}^{r}$ is given by

$$
\begin{aligned}
& \mathscr{B}^{r}(\psi) \\
& =\left(\left(\begin{array}{cc}
2 \partial_{x_{n}} \varphi_{j \mid x_{n}=0^{+}}^{r} & B_{1}^{r} \\
B_{1}^{r \prime} & B_{2}^{r}
\end{array}\right)\left(\begin{array}{l}
\gamma_{1}(\psi) \\
\gamma_{0}(\psi)
\end{array}\right),\left(\begin{array}{l}
\gamma_{1}(\psi) \\
\gamma_{0}(\psi)
\end{array}\right)\right)_{0}, \operatorname{supp}(\psi) \subset\left(0, X_{0}\right) \times \tilde{U}_{j} \times[0,2 \varepsilon),
\end{aligned}
$$

where $B_{1}^{r}, B_{1}^{r \prime} \in \mathscr{D}_{\mathcal{T}}^{1}, B_{2}^{r} \in \mathscr{D}_{\mathcal{T}}^{2}$, with principal symbols $\sigma\left(B_{1}^{r}\right)=\sigma\left(B_{1}^{r \prime}\right)=\left.2 q_{1, j}^{r}\right|_{x_{n}=0^{+}}$ and $\sigma\left(B_{2}^{r}\right)=-\left.2 \partial_{x_{n}} \varphi_{j}^{r} q_{2, j}^{r}\right|_{x_{n}=0^{+}}$with $q_{k, j}^{r}=\left(\phi_{j}^{-1}\right)^{*} q_{k}^{r}, k=1,2$.

Observe that

$$
\left|\left(\left(D_{n} u_{\mathscr{Z}, j}^{r}+L_{1}^{r} u_{\mathscr{Z}, j}^{r}\right)_{\mid x_{n}=0^{+}}, L_{0}^{r} u_{\mathscr{Z}, j}^{r}{\mid x_{n}=0^{+}}_{0}\right)_{0}\right| \lesssim\left|\gamma_{1}\left(u_{\mathscr{Z}, j}^{r}\right)\right|_{0}^{2}+\left|\gamma_{0}\left(u_{\mathscr{Z}, j}^{r}\right)\right|_{1}^{2} .
$$

Thanks to the transmission conditions $\left(\mathrm{TC}_{\bullet}, j\right.$ ) at the interface and the trace relation (4.55) on the "l" side, we shall be able to express $\gamma_{1}\left(u_{\mathscr{Z}, j}^{r}\right)$ through $\gamma_{0}\left(u_{\mathscr{Z}, j}^{r}\right)$ on the "r" side. This will allow us to turn $\mathscr{B}^{r}$ into a quadratic form operating on $\gamma_{0}\left(u_{\mathscr{Z}, j}^{r^{\prime}}\right)$ only. We first formulate $\left(\mathrm{TC}_{\bullet}, j\right)$ in the following manner:

$$
\left\{\begin{array}{l}
\gamma_{0}\left(u_{\mathscr{Z}, j}^{l}\right)=\gamma_{0}\left(u_{\mathscr{Z}, j}^{r}\right)+\theta_{\mathscr{Z}, j}^{l}-\theta_{\mathscr{Z}, j}^{r} \\
\gamma_{1}\left(u_{\mathscr{Z}, j}^{l}\right)=\frac{\delta c_{j}^{s}}{h i c_{j}^{l}} P_{\varphi, j}^{s}\left(\gamma_{0}\left(u_{\mathscr{Z}, j}^{r}\right)-\theta_{\mathscr{Z}, j}^{r}\right)-\beta \gamma_{1}\left(u_{\mathscr{Z}, j}^{r}\right)+k \gamma_{0}\left(u_{\mathscr{Z}, j}^{r}\right)-\tilde{G}_{1}, \\
u_{\mathscr{Z}, j}^{s}=\gamma_{0}\left(u_{\mathscr{Z}, j}^{r}\right)-\theta_{\mathscr{Z}, j}^{r} .
\end{array}\right.
$$


Let $\hat{\chi} \in \mathscr{C}^{\infty}\left(T^{*}\left(\mathbb{R}^{n}\right)\right)$ satisfy the same requirements as $\tilde{\chi}$ with $\tilde{\chi}$ equal to 1 in a neighborhood of the support of $\hat{\chi}$. Since the principal part $b_{-1}^{l}$ does not vanish in a neighborhood of $\operatorname{supp}(\hat{\chi})$ (see (4.56)) one can introduce a parametrix for $\mathrm{Op}_{\mathcal{T}}\left(b^{l}\right)$, say $\mathrm{Op}_{\mathcal{T}}(e)$ with $e \in S_{\mathcal{T}}^{1}$, satisfying

$$
\mathrm{Op}_{\mathcal{T}}(e) \mathrm{Op}_{\mathcal{T}}\left(b^{l}\right)=\mathrm{Op}_{\mathcal{T}}(\hat{\chi})+R, \quad R \in h^{\infty} \Psi_{\mathcal{T}}^{-\infty} .
$$

Note that the principal part of the parametrix $e$ is given by $\sigma(e)=\hat{\chi}\left(\rho_{j}^{l,+}-\rho_{j}^{l,-}\right)_{\mid x_{n}=0^{+}}$. Applying this parametrix to (4.55) gives

$$
\begin{gathered}
\mathrm{Op}_{\mathcal{T}}(e)\left(1-\mathrm{Op}_{\mathcal{T}}\left(a^{l}\right)\right) \gamma_{0}\left(u_{\mathscr{Z}, j}^{l}\right)=\mathrm{Op}_{\mathcal{T}}(\hat{\chi}) \gamma_{1}\left(u_{\mathscr{Z}, j}^{l}\right)+R \gamma_{1}\left(u_{\mathscr{Z}, j}^{l}\right)+\mathrm{Op}_{\mathcal{T}}(e) G_{2}^{l} \\
=\gamma_{1}\left(u_{\mathscr{Z}, j}^{l}\right)+R_{1} D_{x_{n}} v_{\left.\right|_{\mid x_{n}=0^{+}}}^{l}+R_{0} v_{j_{\mid x_{n}=0^{+}}}+\mathrm{Op}_{\mathcal{T}}(e) G_{2}^{l},
\end{gathered}
$$

with $R_{1} \in h^{\infty} \Psi_{\mathcal{T}}^{-\infty}$ and $R_{0} \in h^{\infty} \Psi_{\mathcal{T}}^{-\infty}$, since

$$
\begin{aligned}
\gamma_{1}\left(u_{\mathscr{Z}, j}^{l}\right)= & \mathrm{Op}_{\mathcal{T}}(\hat{\chi}) \gamma_{1}\left(u_{\mathscr{Z}, j}^{l}\right)+\underbrace{\left(1-\mathrm{Op}_{\mathcal{T}}(\hat{\chi})\right) \mathrm{Op}_{\mathcal{T}}\left(\chi_{\mathscr{Z}, j}\right)}_{\in h^{\infty} \Psi_{\mathcal{T}}^{-\infty}} D_{x_{n}} v_{\left.\right|_{\mid x_{n}=0^{+}} ^{l}} \\
& +\underbrace{\left(1-\mathrm{Op}_{\mathcal{T}}(\hat{\chi})\right)\left[D_{x_{n}}, \mathrm{Op}_{\mathcal{T}}\left(\chi_{\mathscr{Z}, j}\right)\right]}_{\in h^{\infty} \Psi_{\mathcal{T}}^{-\infty}} v_{j_{x_{n}=0^{+}}^{l}}^{l}
\end{aligned}
$$

and

$$
R \gamma_{1}\left(u_{\mathscr{Z}, j}^{l}\right)=\underbrace{R \mathrm{Op}_{\mathcal{T}}\left(\chi_{\mathscr{Z}, j}\right)}_{\in h^{\infty} \Psi_{\mathcal{T}}^{-\infty}} D_{x_{n}} v_{\mid x_{n}=0^{+}}^{l}+\underbrace{R\left[D_{x_{n}}, \mathrm{Op}_{\mathcal{T}}\left(\chi_{\mathscr{Z}, j}\right)\right]}_{\in h^{\infty} \Psi_{\mathcal{T}}^{-\infty}} v_{\mid x_{n}=0^{+}}^{l} .
$$

Using the first relation of (4.61) to replace $\gamma_{0}\left(u_{\mathscr{Z}, j}^{l}\right)$ by $\gamma_{0}\left(u_{\mathscr{Z}, j}^{r}\right)$ in (4.62), we obtain

$$
\begin{aligned}
& \mathrm{Op}_{\mathcal{T}}(e)\left(1-\mathrm{Op}_{\mathcal{T}}\left(a^{l}\right)\right)\left(\gamma_{0}\left(u_{\mathscr{Z}, j}^{r}\right)+\theta_{\mathscr{Z}, j}^{l}-\theta_{\mathscr{Z}, j}^{r}\right) \\
& \quad=\gamma_{1}\left(u_{\mathscr{Z}, j}^{l}\right)+R_{1} D_{x_{n}} v_{j_{x_{n}=0^{+}}}^{l}+R_{0} v_{\left.\right|_{x_{n}=0^{+}}}^{l}+\mathrm{Op} \mathcal{T}(e) G_{2}^{l} .
\end{aligned}
$$

Now, applying (4.63) in the second equation of (4.61) yields the following relation between the two traces of $u_{\mathscr{Z}, j}^{r}$ :

$$
\begin{aligned}
\beta \gamma_{1}\left(u_{\mathscr{Z}, j}^{r}\right)= & \left(\frac{\delta c_{j}^{s}}{h i c_{j}^{l}} P_{\varphi, j}^{s}-\mathrm{Op}_{\mathcal{T}}(e)\left(1-\mathrm{Op}_{\mathcal{T}}\left(a^{l}\right)\right)+k\right) \gamma_{0}\left(u_{\mathscr{Z}, j}^{r}\right)-\frac{\delta c_{j}^{s}}{h i c_{j}^{l}} P_{\varphi, j}^{s} \theta_{\mathscr{Z}, j}^{r} \\
& -\mathrm{Op}_{\mathcal{T}}(e)\left(1-\mathrm{Op}_{\mathcal{T}}\left(a^{l}\right)\right)\left(\theta_{\mathscr{Z}, j}^{l}-\theta_{\mathscr{Z}, j}^{r}\right)-\tilde{G}_{1}+\mathrm{Op}_{\mathcal{T}}(e) G_{2}^{l} \\
& +R_{1} D_{x_{n}} v_{\left.\right|_{\mid x_{n}=0^{+}} ^{l}}^{l}+R_{0} v_{j_{\mid x_{n}=0^{+}}^{l} .}
\end{aligned}
$$

This equation can be written in the form

$$
\gamma_{1}\left(u_{\mathscr{Z}, j}^{r}\right)=\Sigma_{\delta} \gamma_{0}\left(u_{\mathscr{Z}, j}^{r}\right)+G_{3},
$$

where

$$
\Sigma_{\delta}=\frac{1}{\beta}\left(\frac{\delta c_{j}^{s}}{h i c_{j}^{l}} P_{\varphi, j}^{s}-\mathrm{Op}_{\mathcal{T}}(e)\left(1-\mathrm{Op}_{\mathcal{T}}\left(a^{l}\right)\right)+k\right)
$$


and by (4.11) and (4.57) the term $G_{3}$ can be estimated as

$$
\begin{aligned}
\left|G_{3}\right|_{0} \lesssim & \frac{\delta}{h}\left|\theta_{\varphi, j}^{r}\right|_{0}+\left|\theta_{\varphi, j}^{l}\right|_{1}+\left|\theta_{\varphi, j}^{r}\right|_{1}+(\delta+h)\left|v_{j}^{s}\right|_{1}+\left|\Theta_{\varphi, j}^{s}\right|_{0} \\
& +h^{-1 / 2}\left(\left\|P_{\varphi, j}^{l} v_{j}^{l}\right\|_{0}+h\left\|v_{j}^{l}\right\|_{1}+h^{2}\left|D_{x_{n}} v_{j_{\mid x_{n}=0^{+}}}^{l}\right|_{0}\right),
\end{aligned}
$$

where we have used the trace formula (1.23) and

$$
P_{\varphi, j}^{s} \theta_{\mathscr{Z}, j}^{r}=\underbrace{P_{\varphi, j}^{s} \mathrm{Op}_{\mathcal{T}}\left(\chi_{\mathscr{Z}, j}\right)}_{\in \Psi_{\mathcal{T}}^{0}} \theta_{\varphi, j}^{r} .
$$

$\operatorname{In} \operatorname{supp}(\hat{\chi})$, from (4.56) the symbol $\sigma_{\delta}$ of $\Sigma_{\delta}$ reads

$$
\sigma_{\delta}=\beta^{-1}\left(-i \frac{\delta c_{j}^{s}}{h c_{j}^{l}} p_{\varphi, j}^{s}-\rho_{j}^{l,+}+k\right)+r \quad \text { with } r \in \delta S_{\mathcal{T}}^{1}+h S_{\mathcal{T}}^{0},
$$

where functions are evaluated at the interface, i.e. $x_{n}=0^{+}$.

Using (4.64) in (4.59), we can now write $\mathscr{B}^{r}\left(u_{\mathscr{Z}, j}^{r}\right)$ as

$$
\begin{aligned}
& \mathscr{B}^{r}\left(u_{\mathscr{Z}, j}^{r}\right)=\left(\left(\begin{array}{cc}
2 \partial_{x_{n}} \varphi_{j \mid x_{n}=0^{+}}^{r} & B_{1}^{r} \\
B_{1}^{r \prime} & B_{2}^{r}
\end{array}\right)\left(\begin{array}{c}
\Sigma_{\delta} \gamma_{0}\left(u_{\mathscr{Z}, j}^{r}\right)+G_{3} \\
\gamma_{0}\left(u_{\mathscr{Z}, j}^{r}\right)
\end{array}\right),\left(\begin{array}{c}
\Sigma_{\delta} \gamma_{0}\left(u_{\mathscr{Z}, j}^{r}\right)+G_{3} \\
\gamma_{0}\left(u_{\mathscr{Z}, j}^{r}\right)
\end{array}\right)\right)_{0} \\
& =\left(\tilde{\Sigma}_{\delta} \gamma_{0}\left(u_{\mathscr{Z}, j}^{r}\right), \gamma_{0}\left(u_{\mathscr{Z}, j}^{r}\right)\right)_{0}+4 \operatorname{Re}\left(\partial_{x_{n}} \varphi_{j}^{r}{ }_{\mid x_{n}=0^{+}} \Sigma_{\delta} \gamma_{0}\left(u_{\mathscr{Z}, j}^{r}\right), G_{3}\right)_{0} \\
& \quad+\left(B_{1}^{r} \gamma_{0}\left(u_{\mathscr{Z}, j}^{r}\right), G_{3}\right)_{0}+\left(B_{1}^{r \prime} G_{3}, \gamma_{0}\left(u_{\mathscr{Z}, j}^{r}\right)_{0}+2\left(\partial_{x_{n}} \varphi_{j}^{r}{ }_{\mid x_{n}=0^{+}} G_{3}, G_{3}\right)_{0},\right.
\end{aligned}
$$

with

$$
\tilde{\Sigma}_{\delta}=2 \Sigma_{\delta}^{*} \partial_{x_{n}} \varphi_{j}^{r}{ }_{\mid x_{n}=0^{+}} \Sigma_{\delta}+\Sigma_{\delta}^{*} B_{1}^{r}+B_{1}^{r \prime} \Sigma_{\delta}+B_{2}^{r} .
$$

The following lemma makes use of condition (4.51) that describes the smallness of the region $\mathscr{Z}$.

Lemma 4.5. Let $\tilde{\sigma}_{\delta}$ be the symbol of $\tilde{\Sigma}_{\delta}$. We have $\hat{\chi} \frac{h^{2}}{h^{2}+\delta^{2}} \tilde{\sigma}_{\delta} \in S_{\mathcal{T}}^{0}$. Moreover, in $\operatorname{supp}(\hat{\chi})$, for $h_{0}>0$ sufficiently small, we have

$$
\frac{h^{2}}{h^{2}+\delta^{2}} \tilde{\sigma}_{\delta} \geq C_{0}>0, \quad 0<h \leq h_{0}
$$

We refer to Appendix C.7 for a proof.

Let $\check{\chi} \in \mathscr{C}_{c}^{\infty}\left(T^{*}\left(\mathbb{R}^{n}\right)\right)$ be equal to 1 on a neighborhood of $\operatorname{supp}\left(\left.\chi_{\mathscr{Z}, j}\right|_{\mid x_{n}=0^{+}}\right)$and such that $\hat{\chi}$ is equal to 1 on a neighborhood of $\operatorname{supp}(\check{\chi})$. We then write

$$
\begin{aligned}
\frac{h^{2}}{h^{2}+\delta^{2}} \tilde{\sigma}_{\delta}=s_{\delta}+r_{\delta}, \quad s_{\delta} & =\frac{h^{2}}{h^{2}+\delta^{2}} \tilde{\sigma}_{\delta} \check{\chi}+C_{0}\left\langle\left(\xi_{0}, \xi^{\prime}\right)\right\rangle^{2}(1-\check{\chi}), \\
r_{\delta} & =\left(\frac{h^{2}}{h^{2}+\delta^{2}} \tilde{\sigma}_{\delta}-C_{0}\left\langle\left(\xi_{0}, \xi^{\prime}\right)\right\rangle^{2}\right)(1-\check{\chi}) .
\end{aligned}
$$


By Lemma 4.5, we have $s_{\delta} \geq C_{0}\left\langle\left(\xi_{0}, \xi^{\prime}\right)\right\rangle^{2}$ and observe that $s_{\delta} \in S_{\mathcal{T}}^{2}$. The Gårding inequality yields, for $h_{0}$ sufficiently small and $0<h \leq h_{0}$,

$$
\left(\tilde{\Sigma}_{\delta} \gamma_{0}\left(u_{\mathscr{Z}, j}^{r}\right), \gamma_{0}\left(u_{\mathscr{Z}, j}^{r}\right)\right)_{0} \geq C\left(1+\frac{\delta^{2}}{h^{2}}\right)\left|\gamma_{0}\left(u_{\mathscr{Z}, j}^{r}\right)\right|_{1}^{2}-C_{N} h^{N}\left|v_{j}^{r} x_{n}=0^{+}\right|_{0}^{2},
$$

as $\operatorname{supp}\left(r_{\delta}\right) \cap \operatorname{supp}(\chi \mathscr{Z}, j)=\emptyset$.

We now estimate the other terms in the expression (4.68). Using the Young inequality, we have, for all $\varepsilon>0$,

$$
\begin{aligned}
\left|\left(B_{1}^{r} \gamma_{0}\left(u_{\mathscr{Z}, j}^{r}\right), G_{3}\right)_{0}\right| & +\left|\left(B_{1}^{r \prime} G_{3}, \gamma_{0}\left(u_{\mathscr{Z}, j}^{r}\right)\right)_{0}\right|+2\left|\left(\partial_{x_{n}} \varphi_{j}^{r}{ }_{\mid x_{n}=0^{+}} G_{3}, G_{3}\right)_{0}\right| \\
& \lesssim \varepsilon\left(1+\frac{\delta^{2}}{h^{2}}\right)\left|\gamma_{0}\left(u_{\mathscr{Z}, j}^{r}\right)\right|_{1}^{2}+\left(1+\frac{h^{2}}{\varepsilon\left(h^{2}+\delta^{2}\right)}\right)\left|G_{3}\right|_{0}^{2} .
\end{aligned}
$$

For the remaining term in (4.68), we have

$$
4\left|\operatorname{Re}\left(\partial_{x_{n}} \varphi_{j \mid x_{n}=0^{+}}^{r} \Sigma_{\delta} \gamma_{0}\left(u_{\mathscr{Z}, j}^{r}\right), G_{3}\right)_{0}\right| \lesssim\left(\frac{\delta}{h}\left|P_{\varphi, j}^{s} \gamma_{0}\left(u_{\mathscr{Z}, j}^{r}\right)\right|_{0}+\left|\gamma_{0}\left(u_{\mathscr{Z}, j}^{r}\right)\right|_{1}\right)\left|G_{3}\right|_{0},
$$

according to (4.65) and (4.67). Taking $\check{\chi}$ as above, we can write

$$
\begin{aligned}
P_{\varphi, j}^{s} \gamma_{0}\left(u_{\mathscr{Z}, j}^{r}\right) & =\underbrace{P_{\varphi, j}^{s} \mathrm{Op}_{\mathcal{T}}(\check{\chi})}_{\in \Psi_{\mathcal{T}}^{0}} \gamma_{0}\left(u_{\mathscr{Z}, j}^{r}\right)+\left.\underbrace{P_{\varphi, j}^{s}\left(1-\mathrm{Op}_{\mathcal{T}}(\check{\chi})\right) \mathrm{Op}_{\mathcal{T}}\left(\chi_{\mathscr{Z}, j}\right)}_{\in h^{\infty} \Psi_{\mathcal{T}}^{-\infty}} v_{j}^{r}\right|_{x_{n}=0^{+}} .
\end{aligned}
$$

Using the Young inequality, for all $\varepsilon>0, N \in \mathbb{N}$ we obtain

$$
\begin{aligned}
4 \mid \operatorname{Re}\left(\partial_{x_{n}} \varphi_{j}^{r}\right. & \left.\Sigma_{\delta x_{n}=0^{+}} \Sigma_{\delta} \gamma_{0}\left(u_{\mathscr{Z}, j}^{r}\right), G_{3}\right)_{0} \mid \\
& \lesssim \varepsilon\left(1+\frac{\delta^{2}}{h^{2}}\right)\left|\gamma_{0}\left(u_{\mathscr{Z}, j}^{r}\right)\right|_{1}^{2}+\frac{1}{\varepsilon}\left|G_{3}\right|_{0}^{2}+\varepsilon C_{N} \delta^{2} h^{N}\left|v_{j \mid x_{n}=0^{+}}^{r}\right|_{0}^{2} .
\end{aligned}
$$

Combining (4.70) and (4.68) with (4.71) and (4.73) gives, for $\varepsilon$ sufficiently small and $\delta \leq \delta_{0}$,

$$
\left(1+\frac{\delta^{2}}{h^{2}}\right)\left|\gamma_{0}\left(u_{\mathscr{Z}, j}^{r}\right)\right|_{1}^{2} \lesssim \mathscr{B}^{r}\left(u_{\mathscr{Z}, j}^{r}\right)+\left|G_{3}\right|_{0}^{2}+C_{N} h^{N}\left|v_{j \mid x_{n}=0^{+}}^{r}\right|_{0}^{2} .
$$

Finally, returning to the Carleman form at the boundary, (4.58), and using (4.60), we obtain, for all $N \in \mathbb{N}$, for $h_{0}$ sufficiently small and $0<h \leq h_{0}$,

$$
\begin{aligned}
h\left\|u_{\mathscr{Z}, j}^{r}\right\|_{1}^{2}+h(1+ & \left.\frac{\delta^{2}}{h^{2}}\right)\left|\gamma_{0}\left(u_{\mathscr{Z}, j}^{r}\right)\right|_{1}^{2} \\
& \lesssim\left\|P_{\varphi, j}^{r} v_{j}^{r}\right\|_{0}^{2}+h\left|G_{3}\right|_{0}^{2}+C_{N} h^{N}\left|v_{j \mid x_{n}=0^{+}}^{r}\right|_{0}^{2}+h^{2}\left|\gamma_{1}\left(u_{\mathscr{Z}, j}^{r}\right)\right|_{0}^{2}
\end{aligned}
$$


Using (4.64), (4.65), (4.67) and (4.72) to estimate $\left|\gamma_{1}\left(u_{\mathscr{Z}, j}^{r}\right)\right|_{0}$ in terms of $\left|\gamma_{0}\left(u_{\mathscr{Z}, j}^{r}\right)\right|_{1}$, we obtain

$$
\left|\gamma_{1}\left(u_{\mathscr{Z}, j}^{r}\right)\right|_{0} \lesssim\left(1+\frac{\delta}{h}\right)\left|\gamma_{0}\left(u_{\mathscr{Z}, j}^{r}\right)\right|_{1}+\left|G_{3}\right|_{0}+C_{N} h^{N}\left|v_{j \mid x_{n}=0^{+}}^{r}\right|_{0} .
$$

Then, replacing $\left|G_{3}\right|_{0}$ by its estimate (4.66) gives, for $h_{0}$ sufficiently small and $0<$ $h \leq h_{0}$,

$$
\begin{aligned}
h\left\|u_{\mathscr{Z}, j}^{r}\right\|_{1}^{2} & +h\left|\gamma_{1}\left(u_{\mathscr{Z}, j}^{r}\right)\right|_{0}^{2}+h\left(1+\frac{\delta^{2}}{h^{2}}\right)\left|\gamma_{0}\left(u_{\mathscr{Z}, j}^{r}\right)\right|_{1}^{2} \\
\lesssim & \left\|P_{\varphi, j}^{r} v_{j}^{r}\right\|_{0}^{2}+h^{2}\left\|v_{j}^{r}\right\|_{1}^{2}+h\left(\delta^{2}+h^{2}\right)\left|v_{j}^{s}\right|_{1}^{2}+\left\|P_{\varphi, j}^{l} v_{j}^{l}\right\|_{0}^{2}+h^{2}\left\|v_{j}^{l}\right\|_{1}^{2} \\
& +\left.h^{4}\left|D_{x_{n}} v_{j}^{l}\right|_{\mid x_{n}=0^{+}}\right|_{0} ^{2}+\frac{\delta^{2}}{h}\left|\theta_{\varphi, j}^{r}\right|_{0}^{2}+h\left|\theta_{\varphi, j}^{l}\right|_{1}^{2}+h\left|\theta_{\varphi, j}^{r}\right|_{1}^{2}+h\left|\Theta_{\varphi, j}^{s}\right|_{0}^{2},
\end{aligned}
$$

using the trace formula (1.23). This is a local version of (4.52). Patching together on $\mathcal{M}_{+}$ such local estimates as we did in (4.16)-(4.21) yields (4.52).

Let us now conclude the proof on the "l" side. The trace equation (4.62) yields

$$
\begin{aligned}
\left|\gamma_{1}\left(u_{\mathscr{Z}, j}^{l}\right)\right|_{0} \leq & \left|\gamma_{0}\left(u_{\mathscr{Z}, j}^{l}\right)\right|_{1}+\left|G_{2}^{l}\right|_{1}+C_{N} h^{N}\left(\left|D_{x_{n}} v_{j \mid x_{n}=0^{+}}^{l}\right|_{0}+\left|v_{\left.\right|_{\mid x_{n}=0^{+}}}^{l}\right|_{0}\right) \\
\leq & \left|\gamma_{0}\left(u_{\mathscr{Z}, j}^{r}\right)\right|_{1}+\left|\theta_{\varphi, j}^{r}\right|_{1}+\left|\theta_{\varphi, j}^{l}\right|_{1}+\left|G_{2}^{l}\right|_{1} \\
& +C_{N} h^{N}\left(\left|D_{x_{n}} v_{j_{x_{n}=0^{+}}}^{l}\right|_{0}+\left|v_{\left.\right|_{\mid x_{n}=0^{+}}}^{l}\right|_{0}\right), \quad N \in \mathbb{N},
\end{aligned}
$$

after using the first relation of (4.61).

Using this last inequality, together with estimate (4.74) on $\left|\gamma_{0}\left(u_{\mathscr{Z}, j}^{r}\right)\right|_{1}$, estimate (4.57) on $\left|G_{2}^{l}\right|_{1}$, (4.54), and the first transmission condition in (4.61), we finally obtain, for $h_{0}$ sufficiently small and $0<h \leq h_{0}$,

$$
\begin{aligned}
\left\|u_{\mathscr{Z}, j}^{l}\right\|_{1}^{2}+ & h\left|\gamma_{0}\left(u_{\mathscr{Z}, j}^{l}\right)\right|_{1}^{2}+h\left|\gamma_{1}\left(u_{\mathscr{Z}, j}^{l}\right)\right|_{0}^{2} \\
\lesssim & \left\|P_{\varphi, j}^{l} v_{j}^{l}\right\|_{0}^{2}+h^{2}\left\|v_{j}^{l}\right\|_{1}^{2}+h^{4}\left|D_{x_{n}} v_{j \mid x_{n}=0^{+}}^{l}\right|_{0}^{2}+\frac{h^{2}}{\delta^{2}+h^{2}}\left(\left\|P_{\varphi, j}^{r} v_{j}^{r}\right\|_{0}^{2}+h^{2}\left\|v_{j}^{r}\right\|_{1}^{2}\right) \\
& +h^{3}\left|v_{j}^{s}\right|_{1}^{2}+\frac{h \delta^{2}}{\delta^{2}+h^{2}}\left|\theta_{\varphi, j}^{r}\right|_{0}^{2}+h\left|\theta_{\varphi, j}^{r}\right|_{1}^{2}+h\left|\theta_{\varphi, j}^{l}\right|_{1}^{2}+\frac{h^{3}}{\delta^{2}+h^{2}}\left|\Theta_{\varphi, j}^{s}\right|_{0}^{2} .
\end{aligned}
$$

This is a local version of (4.53). Patching together on $\mathcal{M}_{+}$such local estimates as we did in (4.16)-(4.21) yields (4.53).

\subsection{Estimate in the region $\mathscr{E}$}

Here, in the region $\mathscr{E}$ (high frequencies), we prove a Carleman estimate for $u_{\mathscr{E}, j}$, and consequently for $\Xi_{\mathscr{E}} v$. Using in this region the ellipticity of $P_{\varphi, j}^{s}$ and the Calderón projector technique for both $P_{\varphi, j}^{r}$ and $P_{\varphi, j}^{l}$, we obtain the following partial estimate. 
Proposition 4.6. Suppose that the weight function $\varphi$ has the properties listed in Section 3.1. Then, for all $\delta_{0}>0$, there exist $C>0$ and $h_{0}>0$ such that, for all $0<\delta \leq \delta_{0}$ and $0<h \leq h_{0}$, and for $v^{r / l} \in \mathscr{C}_{c}^{\infty}\left(\left(0, X_{0}\right) \times S \times[0,2 \varepsilon)\right)$ and $v^{s} \in \mathscr{C}_{c}^{\infty}\left(\left(0, X_{0}\right) \times S\right)$ satisfying (3.9), we have

$$
\begin{aligned}
\left\|\Xi_{\mathscr{E}} v^{r / l}\right\|_{1}^{2}+ & h\left|\Xi_{\mathscr{E}} v_{\mid x_{n}=0^{+}}^{r / l}\right|_{1}^{2}+h\left|D_{x_{n}} \Xi_{\mathscr{E}} v_{\mid x_{n}=0^{+}}^{r / l}\right|_{0}^{2} \\
\leq & C\left(\left\|P_{\varphi}^{r} v^{r}\right\|_{0}^{2}+h^{2}\left\|v^{r}\right\|_{1}^{2}+h^{4}\left|D_{x_{n}} v_{\mid x_{n}=0^{+}}^{r}\right|_{0}^{2}+\left\|P_{\varphi}^{l} v^{l}\right\|_{0}^{2}+h^{2}\left\|v^{l}\right\|_{1}^{2}\right. \\
& \left.+h^{4}\left|D_{x_{n}} v_{\mid x_{n}=0^{+}}^{l}\right|_{0}^{2}+h^{3}\left|v^{s}\right|_{1}^{2}+h\left|\Theta_{\varphi}^{s}\right|_{0}^{2}+h\left|\theta_{\varphi}^{r}\right|_{1}^{2}+h\left|\theta_{\varphi}^{l}\right|_{1}^{2}\right) .
\end{aligned}
$$

Proof. The function $u_{\mathscr{E}, j}$ satisfies $\left(\mathrm{TC}_{\bullet}, j\right.$ ), with $\bullet=\mathscr{E}$. On each side, the root configuration described in Lemma 3.6 (and represented in Figure 3c) allows us to apply the Calderón projector technique as in [LR97, LR10]. Since the two roots are separated by the real axis we only obtain one relation between the two traces at the interface: according to [LR10, Eq. (2.37)], we have

$$
\begin{aligned}
\left\|u_{\mathscr{E}, j}^{r / l}\right\|_{1} \lesssim & \left\|P_{\varphi, j}^{r / l} v_{j}^{r / l}\right\|_{0}+h\left\|v_{j}^{r / l}\right\|_{1}+h^{1 / 2}\left|\gamma_{0}\left(u_{\mathscr{E}, j}^{r / l}\right)\right|_{1}+h^{1 / 2}\left|\gamma_{1}\left(u_{\mathscr{E}, j}^{r / l}\right)\right|_{0} \\
& +\left.h^{2}\left|D_{x_{n}} v_{j}^{r / l}\right|_{n=0^{+}}\right|_{0},
\end{aligned}
$$

together with one relation between the two traces [LR10, Eq. (2.38)]:

$$
\left(1-\mathrm{Op}_{\mathcal{T}}\left(a^{r / l}\right)\right) \gamma_{0}\left(u_{\mathscr{E}, j}^{r / l}\right)=\mathrm{Op}_{\mathcal{T}}\left(b^{r / l}\right) \gamma_{1}\left(u_{\mathscr{E}, j}^{r / l}\right)+G_{2}^{r / l} .
$$

In this last expression, $a^{r / l} \in S_{\mathcal{T}}^{0}$ and $b^{r / l} \in S_{\mathcal{T}}^{-1}$ have principal parts respectively

$$
a_{0}^{r / l}=-\left.\left(\tilde{\chi} \frac{\rho_{j}^{r / l,-}}{\rho_{j}^{r / l,+}-\rho_{j}^{r / l,-}}\right)\right|_{x_{n}=0^{+}} \quad \text { and } \quad b_{-1}^{r / l}=\left.\left(\tilde{\chi} \frac{1}{\rho_{j}^{r / l,+}-\rho_{j}^{r / l,-}}\right)\right|_{x_{n}=0^{+}},
$$

where $\rho_{j}^{l, \pm}$ are the roots of $p_{\varphi, j}^{l}$ (i.e. $\rho_{j}^{l, \pm}=\left(\phi_{j}^{-1}\right)^{*} \rho^{l, \pm}$ with $\rho^{l, \pm}$ described in Lemma 3.6) and $\tilde{\chi} \in \mathscr{C}^{\infty}\left(T^{*}\left(\mathbb{R}^{n}\right)\right)$ is equal to 1 in the neighborhood of the support of $\chi_{\mathscr{E}}, j_{\mid x_{n}=0^{+}}$, with support in

$$
\left(\left(\phi_{j}^{-1}\right)^{*} \mathscr{E}\right) \cap\left\{x_{n}=0\right\}=\left\{\left(x_{0}, \phi_{j}(y) ; \xi_{0},{ }^{t} d \phi_{j}^{-1}\left(\phi_{j}(y)\right) \eta\right) ;\left(x_{0}, y, 0 ; \xi_{0}, \eta\right) \in \mathscr{E}\right\} .
$$

The remainder $G_{2}^{r / l}$ satisfies

$$
\left|G_{2}^{r / l}\right|_{1} \lesssim h^{-1 / 2}\left(\left\|P_{\varphi, j}^{r / l} v_{j}^{r / l}\right\|_{0}+h\left\|v_{j}^{r / l}\right\|_{1}+h^{2}\left|D_{x_{n}} v_{j \mid x_{n}=0^{+}}^{r / l}\right| 0\right) .
$$

The principal part of $b^{r / l}$ satisfies

$$
b_{-1}^{r / l} \geq C\left\langle\left(\xi_{0}, \xi^{\prime}\right)\right\rangle^{-1} \quad \text { in } \operatorname{supp}(\tilde{\chi}),
$$

as $\rho_{j}^{r / l,+}$ and $\rho_{j}^{r / l,-}$ are tangential symbols of order one such that $\rho_{j}^{r / l,+}-\rho_{j}^{r / l,-}$ does not vanish in a neighborhood of $\operatorname{supp}(\tilde{\chi})$. Let $\hat{\chi} \in \mathscr{C}^{\infty}\left(T^{*}\left(\mathbb{R}^{n}\right)\right)$ satisfy the same requirements as $\tilde{\chi}$ with $\tilde{\chi}$ equal to 1 in a neighborhood of the support of $\hat{\chi}$. We can introduce parametrices for $\operatorname{Op}_{\mathcal{T}}\left(b^{r / l}\right)$, say $\mathrm{Op}_{\mathcal{T}}\left(e^{r / l}\right)$ with $e^{r / l} \in S_{\mathcal{T}}^{1}$, satisfying

$$
\mathrm{Op}_{\mathcal{T}}\left(e^{r / l}\right) \mathrm{Op}_{\mathcal{T}}\left(b^{r / l}\right)=\mathrm{Op}_{\mathcal{T}}(\hat{\chi})+R^{r / l}, \quad R^{r / l} \in h^{\infty} \Psi_{\mathcal{T}}^{-\infty} .
$$


Note that the principal parts of the parametrices $e^{r / l}$ are given by $\sigma\left(e^{r / l}\right)=$ $\hat{\chi}\left(\rho_{j}^{r / l,+}-\rho_{j}^{r / l,-}\right){ }_{\mid x_{n}=0^{+}}$.

Applying these parametrices to (4.77) and arguing as in (4.50) gives

$$
\begin{aligned}
\mathrm{Op}_{\mathcal{T}}\left(e^{r / l}\right)(1 & \left.-\mathrm{Op}_{\mathcal{T}}\left(a^{r / l}\right)\right) \gamma_{0}\left(u_{\mathscr{E}, j}^{r / l}\right) \\
& =\gamma_{1}\left(u_{\mathscr{E}, j}^{r / l}\right)+R_{1}^{r / l} D_{x_{n}} v_{j \mid x_{n}=0^{+}}^{r / l}+R_{0}^{r / l} v_{j}^{r / l}{ }_{\mid x_{n}=0^{+}}+\mathrm{Op}_{\mathcal{T}}\left(e^{r / l}\right) G_{2}^{r / l}
\end{aligned}
$$

with $R_{0}^{r / l}, R_{1}^{r / l} \in h^{\infty} \Psi_{\mathcal{T}}^{-\infty}$. This yields the following estimate of $\gamma_{1}\left(u_{\mathscr{E}, j}^{r / l}\right)$ in terms of $\gamma_{0}\left(u_{\mathscr{E}, j}^{r / l}\right)$ :

$$
\begin{aligned}
& \left|\gamma_{1}\left(u_{\mathscr{E}, j}^{r / l}\right)\right|_{0} \\
& \quad \lesssim\left|\gamma_{0}\left(u_{\mathscr{E}, j}^{r / l}\right)\right|_{1}+\left|G_{2}^{r / l}\right|_{1}+C_{N} h^{N}\left(\left|D_{x_{n}} v_{j \mid x_{n}=0^{+}}^{r / l}\right|_{0}+\left|v_{j \mid x_{n}=0^{+}}^{r / l}\right|_{0}\right), \quad N \in \mathbb{N} .
\end{aligned}
$$

On the other hand, replacing $u_{\mathscr{E}, j}^{s}$ in the first equation of $\left(\mathrm{TC}_{\bullet, j}\right)$ by its expression in the second equation of $\left(\mathrm{TC}_{\bullet}, j\right)$ gives

$$
\delta \frac{c_{j}^{s}}{i c_{j}^{l}} P_{\varphi, j}^{s}\left(\gamma_{0}\left(u_{\mathscr{E}, j}^{r}\right)-\theta_{\mathscr{E}, j}^{r}\right)=h\left(\gamma_{1}\left(u_{\mathscr{E}, j}^{l}\right)+\beta \gamma_{1}\left(u_{\mathscr{E}, j}^{r}\right)-k \gamma_{0}\left(u_{\mathscr{E}, j}^{r}\right)+\tilde{G}_{1}\right) .
$$

By using (4.80) and the first equation of $\left(\mathrm{TC}_{\bullet}, j\right)$, this yields

$$
\Omega_{\delta} \gamma_{0}\left(u_{\mathscr{E}, j}^{r}\right)=G_{3}
$$

with

$\Omega_{\delta}=\delta \frac{c_{j}^{s}}{i c_{j}^{l}} P_{\varphi, j}^{s}+h\left(k-\beta \mathrm{Op}_{\mathcal{T}}\left(e^{r}\right)\left(1-\mathrm{Op}_{\mathcal{T}}\left(a^{r}\right)\right)-\mathrm{Op}_{\mathcal{T}}\left(e^{l}\right)\left(1-\mathrm{Op}_{\mathcal{T}}\left(a^{l}\right)\right)\right)$

and

$$
\begin{aligned}
& G_{3}=\delta \frac{c_{j}^{s}}{i c_{j}^{l}} P_{\varphi, j}^{s} \theta_{\mathscr{E}, j}^{r}+h \tilde{G}_{1}-h \beta\left(R_{1}^{r} D_{x_{n}} v_{j \mid x_{n}=0^{+}}^{r}+R_{0}^{r} v_{j}^{r}{ }_{\mid x_{n}=0^{+}}+\operatorname{Op}_{\mathcal{T}}\left(e^{r}\right) G_{2}^{r}\right) \\
& -h\left(R_{1}^{l} D_{x_{n}} v_{j \mid x_{n}=0^{+}}^{l}+R_{0}^{l} v_{j \mid x_{n}=0^{+}}^{l}+\operatorname{Op}_{\mathcal{T}}\left(e^{l}\right) G_{2}^{l}+\operatorname{Op} \mathcal{T}\left(e^{l}\right)\left(1-\operatorname{Op}_{\mathcal{T}}\left(a^{l}\right)\right)\left(\theta_{\mathscr{E}, j}^{r}-\theta_{\mathscr{E}, j}^{l}\right)\right)
\end{aligned}
$$

Here, we introduce a class of pseudo-differential operators adapted to the operator $\Omega_{\delta}$ in order to perform uniform estimates in the singular limit $\delta \rightarrow 0^{+}$. On the tangential phase space $W=T^{*}\left(\mathbb{R}^{n}\right)$, we define the order function

$$
\Lambda^{2}:=\frac{\delta}{\delta+h}\left\langle\left(\xi_{0}, \xi^{\prime}\right)\right\rangle^{2}+\frac{h}{\delta+h}\left\langle\left(\xi_{0}, \xi^{\prime}\right)\right\rangle
$$

associated with the metric

$$
g_{W}=\left|d\left(x_{0}, x^{\prime}\right)\right|^{2}+\frac{\left|d\left(\xi_{0}, \xi^{\prime}\right)\right|^{2}}{\left\langle\left(\xi_{0}, \xi^{\prime}\right)\right\rangle^{2}} .
$$


Lemma 4.7. The order function $\Lambda$ is admissible, i.e., slowly varying and temperate.

We refer to Appendix C.8 for a proof. For a review of these notions see [Hör79] or [Hör85a, Sec. 18.4-5], or the recent monograph [Ler10, Def. 2.2.4 and 2.2.15]. Thanks to the previous lemma, we can define a proper Hörmander-class calculus. We now prove that $\Omega_{\delta}$ is elliptic in this class.

We set

$$
\omega_{\delta}=\delta \frac{c_{j}^{s}}{i c_{j}^{l}} p_{\varphi, j}^{s}+h\left(k-\beta \hat{\chi} \rho_{j}^{r,+}-\hat{\chi} \rho_{j}^{l,+}\right) .
$$

We have $(\delta+h)^{-1} \omega_{\delta} \in S_{\mathcal{T}}\left(\Lambda^{2}, g_{W}\right)$. By (4.78) we see that

$$
\Omega_{\delta}-\mathrm{Op}_{\mathcal{T}}\left(\omega_{\delta}\right) \in h \delta \Psi_{\mathcal{T}}^{1}+h^{2} \Psi_{\mathcal{T}}^{0} \subset(h+\delta) \Psi_{\mathcal{T}}\left(h \Lambda^{2} /\left\langle\left(\xi_{0}, \xi^{\prime}\right)\right\rangle, g_{W}\right) .
$$

From the definition of $k$ in (4.10) this gives

$$
\operatorname{Im}\left(\omega_{\delta}\right)=-\delta \frac{c_{j}^{s}}{c_{j}^{l}} \operatorname{Re}\left(p_{\varphi, j}^{s}\right)-h\left(\partial_{x_{n}} \varphi_{j \mid x_{n}=0^{+}}^{l}+\beta \partial_{x_{n}} \varphi_{j \mid x_{n}=0^{+}}^{r}+\hat{\chi} \operatorname{Im}\left(\rho_{j}^{l,+}+\beta \rho_{j}^{r,+}\right)\right) .
$$

In this expression, we have

$$
\operatorname{Re}\left(p_{\varphi, j}^{s}\right) \geq C\left\langle\left(\xi_{0}, \xi^{\prime}\right)\right\rangle^{2} \quad \text { on supp } \hat{\chi}
$$

by Proposition 3.5 (see also the position of $\operatorname{Char}\left(P_{\varphi, j}^{s}\right)$ in Figure 4). Next, in the region where $\hat{\chi}=1$ we have

$$
\begin{aligned}
\partial_{x_{n}} \varphi_{j \mid x_{n}=0^{+}}^{l}+\beta \partial_{x_{n}} \varphi_{j \mid x_{n}=0^{+}}^{r}+\operatorname{Im}\left(\rho_{j}^{l,+}+\beta \rho_{j}^{r,+}\right) \\
\quad=\frac{1}{2} \operatorname{Im}\left(\rho_{j}^{l,+}-\rho_{j}^{l,-}\right)+\frac{1}{2} \beta \operatorname{Im}\left(\rho_{j}^{r,+}-\rho_{j}^{r,-}\right) \geq C\left\langle\left(\xi_{0}, \xi^{\prime}\right)\right\rangle,
\end{aligned}
$$

as $\partial_{x_{n}} \varphi_{j}^{r / l}=-\frac{1}{2} \operatorname{Im}\left(\rho_{j}^{l,+}+\rho_{j}^{l,-}\right)$ and by Lemma 3.8. Estimates (4.86) and (4.87) yield

$$
\left|\omega_{\delta}\right| \geq C(\delta+h) \Lambda^{2}
$$

in the region where $\hat{\chi}=1$. There, the symbol $(\delta+h)^{-1} \omega_{\delta}$ is elliptic in the class $S_{\mathcal{T}}\left(\Lambda^{2}, g_{W}\right)$. Hence, there exists $l \in S_{\mathcal{T}}\left(\Lambda^{-2}, g_{W}\right)$ (with principal part $\check{\chi} \omega_{\delta}^{-1}$ ) such that

$$
\mathrm{Op}_{\mathcal{T}}(l)(\delta+h)^{-1} \Omega_{\delta}=\mathrm{Op}_{\mathcal{T}}(\check{\chi})+R, \quad R \in h^{\infty} \Psi_{\mathcal{T}}^{-\infty},
$$

by (4.85), for some $\check{\chi} \in \mathscr{C}^{\infty}\left(T^{*}\left(\mathbb{R}^{n}\right)\right)$ equal to 1 on a neighborhood of $\operatorname{supp}\left(\chi_{\mathscr{E}}, j_{\mid x_{n}=0^{+}}\right)$ and such that $\hat{\chi}$ is equal to 1 on a neighborhood of $\operatorname{supp}(\check{\chi})$.

Applying this parametrix to (4.82) gives

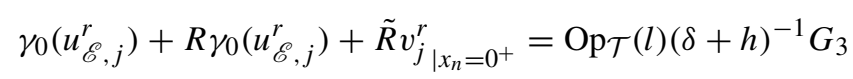

with $R \in h^{\infty} \Psi_{\mathcal{T}}^{-\infty}$ and $\tilde{R}=\operatorname{Op}_{\mathcal{T}}(\check{\chi}-1) \operatorname{Op}_{\mathcal{T}}\left(\chi_{\mathscr{E}, j_{\mid x_{n}=0^{+}}}\right) \in h^{\infty} \Psi_{\mathcal{T}}^{-\infty}$. 
We estimate

$$
\left|\mathrm{Op}_{\mathcal{T}}(l)(\delta+h)^{-1} G_{3}\right|_{1}=\left|\mathrm{Op}_{\mathcal{T}}\left(\left\langle\left(\xi_{0}, \xi^{\prime}\right)\right\rangle\right) \mathrm{Op}_{\mathcal{T}}(l)(\delta+h)^{-1} G_{3}\right|_{0}
$$

with

$\operatorname{Op}_{\mathcal{T}}\left(\left\langle\left(\xi_{0}, \xi^{\prime}\right)\right\rangle\right) \operatorname{Op}_{\mathcal{T}}(l)(\delta+h)^{-1} \in \Psi_{\mathcal{T}}\left(\frac{\left\langle\left(\xi_{0}, \xi^{\prime}\right)\right\rangle}{(\delta+h) \Lambda^{2}}, g_{W}\right)=\Psi_{\mathcal{T}}\left(\frac{1}{\delta\left\langle\left(\xi_{0}, \xi^{\prime}\right)\right\rangle+h}, g_{W}\right)$.

We thus obtain, as $\operatorname{Op}_{\mathcal{T}}\left(\frac{1}{\delta\left\langle\left(\xi_{0}, \xi^{\prime}\right)\right\rangle+h}\right)$ is a Fourier multiplier,

$$
\left|\mathrm{Op}_{\mathcal{T}}(l)(\delta+h)^{-1} G_{3}\right|_{1} \lesssim\left|\mathrm{Op}_{\mathcal{T}}\left(\frac{1}{\delta\left\langle\left(\xi_{0}, \xi^{\prime}\right)\right\rangle+h}\right) G_{3}\right|_{0}
$$

In view of (4.84), this yields

$$
\begin{aligned}
& \left|\mathrm{Op}_{\mathcal{T}}(l)(\delta+h)^{-1} G_{3}\right|_{1} \\
& \quad \lesssim\left|P_{\varphi, j}^{s} \theta_{\mathscr{E}, j}^{r}\right|_{-1}+\left|\operatorname{Op} \mathcal{T}\left(\frac{h}{\delta\left\langle\left(\xi_{0}, \xi^{\prime}\right)\right\rangle+h}\right) \tilde{G}_{1}\right|_{0}+\left|R_{1}^{r} D_{x_{n}} v_{j \mid x_{n}=0^{+}}^{r}\right|_{0}+\left|R_{0}^{r} v_{j \mid x_{n}=0^{+}}^{r}\right|_{0} \\
& \quad+\left|R_{1}^{l} D_{x_{n}} v_{j \mid x_{n}=0^{+}}^{l}\right|_{0}+\left|R_{0}^{l} v_{j_{\mid x_{n}=0^{+}}}^{l}\right|_{0}+\left|G_{2}^{r}\right|_{1}+\left|G_{2}^{l}\right|_{1}+\left|\theta_{\mathscr{E}, j}^{r}\right|_{1}+\left|\theta_{\mathscr{E}, j}^{l}\right|_{1} .
\end{aligned}
$$

Since

$$
\left|\operatorname{Op}_{\mathcal{T}}\left(\frac{h}{\delta\left\langle\left(\xi_{0}, \xi^{\prime}\right)\right\rangle+h}\right) \tilde{G}_{1}\right|_{0} \leq \frac{h}{\delta+h}\left|\tilde{G}_{1}\right|_{0},
$$

and by (4.11), (4.79), and the trace formula (1.23), using also $P_{\varphi, j}^{s} \in \Psi_{\mathcal{T}}^{2}$ gives

$$
\begin{aligned}
\left|\mathrm{Op}_{\mathcal{T}}(l)(\delta+h)^{-1} G_{3}\right|_{1} \lesssim & h\left|v_{j}^{s}\right|_{1}+h^{-1 / 2}\left(\left\|P_{\varphi, j}^{r} v_{j}^{r}\right\|_{0}+h\left\|v_{j}^{r}\right\|_{1}+h^{2}\left|D_{x_{n}} v_{j \mid x_{n}=0^{+}}^{r}\right|_{0}\right) \\
& +h^{-1 / 2}\left(\left\|P_{\varphi, j}^{l} v_{j}^{l}\right\|_{0}+h\left\|v_{j}^{l}\right\|_{1}+h^{2}\left|D_{x_{n}} v_{j \mid x_{n}=0^{+}}^{l}\right|_{0}\right) \\
& +\left|\theta_{\varphi, j}^{r}\right|_{1}+\left|\theta_{\varphi, j}^{l}\right|_{1}+\left|\Theta_{\varphi, j}^{s}\right|_{0} .
\end{aligned}
$$

By (4.88), the transmission conditions (TC•,j), and (4.80) that represents $\gamma_{1}\left(u_{\mathscr{E}, j}^{r / l}\right)$ as a function of $\gamma_{0}\left(u_{\mathscr{E}, j}^{r / l}\right)$ (thanks to the Calderón projectors), we obtain

$$
\begin{aligned}
& h^{1 / 2}\left|\gamma_{0}\left(u_{\mathscr{E}, j}^{r / l}\right)\right|_{1}+h^{1 / 2}\left|\gamma_{1}\left(u_{\mathscr{E}, j}^{r / l}\right)\right|_{0} \lesssim h^{3 / 2}\left|v_{j}^{s}\right|_{1}+\left\|P_{\varphi, j}^{r} v_{j}^{r}\right\|_{0}+h\left\|v_{j}^{r}\right\|_{1}+h^{2}\left|D_{x_{n}} v_{j \mid x_{n}=0^{+}}^{r}\right|_{0} \\
& \quad+\left\|P_{\varphi, j}^{l} v_{j}^{l}\right\|_{0}+h\left\|v_{j}^{l}\right\|_{1}+h^{2}\left|D_{x_{n}} v_{j \mid x_{n}=0^{+}}^{l}\right|_{0}+h^{1 / 2}\left|\theta_{\varphi, j}^{r}\right|_{1}+h^{1 / 2}\left|\theta_{\varphi, j}^{l}\right|_{1}+h^{1 / 2}\left|\Theta_{\varphi, j}^{s}\right|_{0} .
\end{aligned}
$$

Inserting these estimates in (4.76) we obtain a local version of (4.75). Patching together on $\mathcal{M}_{+}$such local estimates as we did in (4.16)-(4.21) yields the result.

\subsection{A semiglobal Carleman estimate: proof of Theorem 1.2}

In this section, we explain how we can patch together the four microlocal estimates of Propositions 4.1, 4.3, 4.4 and 4.6 to obtain a global Carleman estimate in a neighborhood of $S$, and prove Theorem 1.2. 
First, let us introduce some notation. We set

$$
\begin{aligned}
\operatorname{BT}(w) & :=h\left|w_{\mid x_{n}=0^{+}}\right|_{1}^{2}+h\left|D_{x_{n}} w_{\mid x_{n}=0^{+}}\right|_{0}^{2}, \\
\operatorname{RHS}^{r / l}(w) & :=\left\|P_{\varphi}^{r / l} w\right\|_{0}^{2}+h^{2}\|w\|_{1}^{2}+h^{4}\left|D_{x_{n}} w_{\mid x_{n}=0^{+}}\right|_{0}^{2}, \\
R_{\theta} & :=h\left|\Theta_{\varphi}^{s}\right|_{0}^{2}+h\left|\theta_{\varphi}^{r}\right|_{1}^{2}+h\left|\theta_{\varphi}^{l}\right|_{1}^{2} .
\end{aligned}
$$

This allows us to formulate concisely the four microlocal estimates of Propositions 4.1, 4.3, 4.4 and 4.6:

$$
\begin{gathered}
\left\|\Xi_{\mathscr{G}} v^{r}\right\|_{1}^{2}+\mathrm{BT}\left(\Xi_{\mathscr{G}} v^{r}\right) \lesssim \operatorname{RHS}^{r}\left(v^{r}\right), \\
\varepsilon h\left\|\Xi_{\mathscr{G}} v^{l}\right\|_{1}^{2}+\varepsilon \operatorname{BT}\left(\Xi_{\mathscr{G}} v^{l}\right) \lesssim\left(1+\frac{\delta^{2}}{h^{2}}\right)\left(\varepsilon\left\|\zeta P_{\varphi}^{r} v^{r}\right\|_{0}^{2}+\varepsilon h^{4}\left|D_{x_{n}} v_{\mid x_{n}=0^{+}}^{r}\right|_{0}^{2}+\varepsilon h^{4}\left\|v^{r}\right\|_{1}^{2}\right) \\
+\varepsilon \operatorname{RHS}^{l}\left(v^{l}\right)+\varepsilon\left(h^{2}+\delta^{2}\right)\left\|\Xi_{\mathscr{G} \mathscr{F}} v^{r}\right\|_{1}^{2}+\varepsilon h\left(h^{2}+\delta^{2}\right)\left|v^{s}\right|_{1}^{2}+\varepsilon R_{\theta}+\varepsilon \frac{\delta^{2}}{h}\left|\theta_{\varphi}^{r}\right|_{0}^{2}, \\
\left\|\Xi_{\mathscr{F}} v^{r}\right\|_{1}^{2}+\operatorname{BT}\left(\Xi_{\mathscr{F}} v^{r}\right) \lesssim \operatorname{RHS}^{r}\left(v^{r}\right), \\
\left\|\Xi_{\mathscr{F}} v^{l}\right\|_{1}^{2}+\operatorname{BT}\left(\Xi_{\mathscr{F}} v^{l}\right) \lesssim \operatorname{RHS}^{l}\left(v^{l}\right)+\operatorname{RHS}^{r}\left(v^{r}\right)+R_{\theta} . \\
\varepsilon h\left\|\Xi_{\mathscr{Z}} v^{r}\right\|_{1}^{2}+\varepsilon \operatorname{BT}\left(\Xi_{\mathscr{Z}} v^{r}\right) \lesssim \varepsilon \operatorname{RHS}^{r}\left(v^{r}\right)+\varepsilon \operatorname{RHS}^{l}\left(v^{l}\right) \\
\quad+\varepsilon \operatorname{sh}^{l}\left(h^{2}+\delta^{2}\right)\left|v^{s}\right|_{1}^{2}+\varepsilon \frac{\delta^{2}}{h}\left|\theta_{\varphi}^{r}\right|_{0}^{2}+\varepsilon R_{\theta}, \\
\left\|\Xi_{\mathscr{Z}} v^{l}\right\|_{1}^{2}+\operatorname{BT}\left(\Xi_{\mathscr{Z}} v^{l}\right) \lesssim \operatorname{RHS}^{l}\left(v^{l}\right)+h^{3}\left|v^{s}\right|_{1}^{2}+\frac{h^{2}}{\delta^{2}+h^{2}} \operatorname{RHS}^{r}\left(v^{r}\right)+R_{\theta} . \\
\left\|\Xi_{\mathscr{E}} v^{r / l}\right\|_{1}^{2}+\operatorname{BT}\left(\Xi_{\mathscr{E}} v^{r / l}\right) \lesssim \operatorname{RHS}^{l}\left(v^{l}\right)+\operatorname{RHS}^{r}\left(v^{r}\right)+h^{3}\left|v^{s}\right|_{1}^{2}+R_{\theta} .
\end{gathered}
$$

To derive the final Carleman estimate we need to sum together these microlocal estimates and many terms on the r.h.s. need to be absorbed by those on the 1.h.s. This is a standard procedure usually making use of the powers of the parameter $h$ in front of these terms and choosing $h$ sufficiently small. Note, however, that some powers of $h$ are critical here so that the related (framed) terms on the right-hand sides cannot be absorbed directly. To overcome this problem, we have multiplied the two relevant equations by a small parameter $\varepsilon>0$ whose value is independent of $h$ and $\delta$.

Note that these three atypical terms are the reason for the introduction of the microlocal region $\mathscr{F}$ (cf. the microlocal regions used in [LR10]). In fact, the microlocal region $\mathscr{F}$ acts as a buffer: as $\mathscr{F}$ is an elliptic region for both the operators $P_{\varphi}^{r / l}$, it provides terms on the 1.h.s. of the associated microlocal estimates of better quality than those obtained in the regions $\mathscr{G}$ and $\mathscr{Z}$ (compare the powers of $h$ in the 1.h.s. terms of these estimates).

Observe that the property $\chi_{\mathscr{E}}+\chi_{\mathscr{Z}}+\chi_{\mathscr{F}}+\chi_{\mathscr{G}}=1$ implies (see Section 3.6)

$$
\Xi_{\mathscr{G}, j}+\Xi_{\mathscr{F}, j}+\Xi_{\mathscr{Z}, j}+\Xi_{\mathscr{E}, j}=\zeta_{j}\left(x_{0}, y\right) .
$$

As a consequence of the definition of the operators $\Xi_{\bullet}, \bullet=\mathscr{E}, \mathscr{Z}, \mathscr{F}, \mathscr{G}$, given in (3.24)(3.25), this yields

$$
\Xi_{\mathscr{E}}+\Xi_{\mathscr{Z}}+\Xi_{\mathscr{F}}+\Xi_{\mathscr{G}}=\zeta^{1}\left(x_{0}\right)
$$


We now treat the three atypical terms and use the small parameter $\varepsilon$.

As $\operatorname{supp}\left(v^{s}\right) \subset\left(\alpha_{0}, X_{0}-\alpha_{0}\right) \times S$ (see the statement of Theorem 1.2 and Section 3.3), by (4.96), and using the transmission conditions (3.9), we have

$$
\begin{aligned}
v^{s} & =\zeta^{1} v^{s}=\Xi_{\mathscr{E}} v^{s}+\Xi_{\mathscr{Z}} v^{s}+\Xi_{\mathscr{F}} v^{s}+\Xi_{\mathscr{G}} v^{s} \\
& =\Xi_{\mathscr{E}} v^{l}-\Xi_{\mathscr{E}} \theta_{\varphi}^{l}+\Xi_{\mathscr{Z}} v^{l}-\Xi_{\mathscr{Z}} \theta_{\varphi}^{l}+\Xi_{\mathscr{F}} v^{l}-\Xi_{\mathscr{F}} \theta_{\varphi}^{l}+\Xi_{\mathscr{G}} v^{r}-\Xi_{\mathscr{G}} \theta_{\varphi}^{r} \quad \text { at } x_{n}=0^{+} .
\end{aligned}
$$

Hence, for $\delta \leq \delta_{0}$ and $h \leq h_{0}$ we can estimate the two atypical terms involving $v^{s}$ in (4.90) and (4.93) as

$$
\varepsilon h \delta^{2}\left|v^{s}\right|_{1}^{2} \lesssim \varepsilon h\left|\Xi_{\mathscr{E}} v^{l}\right|_{1}^{2}+\varepsilon h\left|\Xi_{\mathscr{Z}} v^{l}\right|_{1}^{2}+\varepsilon h\left|\Xi_{\mathscr{F}} v^{l}\right|_{1}^{2}+\varepsilon h\left|\Xi_{\mathscr{G}} v^{r}\right|_{1}^{2}+\varepsilon R_{\theta} .
$$

When summing all the estimates (4.89)-(4.95) together and taking $\varepsilon$ sufficiently small, the four terms $\varepsilon h\left|\Xi_{\mathscr{E}} v^{l}\right|_{1}^{2}, \varepsilon h\left|\Xi_{\mathscr{Z}} v^{l}\right|_{1}^{2}, \varepsilon h\left|\Xi_{\mathscr{F}} v^{l}\right|_{1}^{2}, \varepsilon h\left|\Xi_{\mathscr{G}} v^{r}\right|_{1}^{2}$ can be absorbed by the 1.h.s. of (4.95), (4.94), (4.92), and (4.89) respectively.

The remaining atypical term is in (4.90):

$$
\varepsilon\left(h^{2}+\delta^{2}\right)\left\|\Xi_{\mathscr{G} \mathscr{F}} v^{r}\right\|_{1}^{2} \lesssim \varepsilon\left\|\Xi_{\mathscr{G} \mathscr{F}} v^{r}\right\|_{1}^{2} .
$$

We choose $\zeta^{4} \in \mathscr{C}_{c}^{\infty}\left(0, X_{0}\right)$ such that $\zeta^{4}=1$ on a neighborhood of $\left(\alpha_{0}, X_{0}-\alpha_{0}\right), \zeta^{1}=1$ on a neighborhood of $\operatorname{supp}\left(\zeta^{4}\right)$ and $0 \leq \zeta^{4} \leq 1$. Since $\operatorname{supp}\left(v^{r}\right) \subset\left(\alpha_{0}, X_{0}-\alpha_{0}\right) \times S \times$ $[0,2 \varepsilon)$, we have

$$
\Xi_{\mathscr{G} \mathscr{F}} v^{r}=\Xi_{\mathscr{G} \mathscr{F}}\left(\Xi_{\mathscr{G}}+\Xi_{\mathscr{F}}\right) v^{r}+\Xi_{\mathscr{G} \mathscr{F}}\left(1-\Xi_{\mathscr{G}}-\Xi_{\mathscr{F}}\right) \zeta^{4} v^{r}
$$

From Proposition B.14 and Proposition B.10, the principal symbol of the operator $\Xi_{\mathscr{G} \mathscr{F}}\left(1-\Xi_{\mathscr{G}}-\Xi_{\mathscr{F}}\right) \zeta^{4}$ is

$$
\zeta^{2} \chi_{\mathscr{G} \mathscr{F}}\left(1-\zeta^{1}\left(\chi_{\mathscr{G}}+\chi_{\mathscr{F}}\right)\right) \zeta^{4}=\zeta^{2} \chi_{\mathscr{G} \mathscr{F}}\left(1-\left(\chi_{\mathscr{G}}+\chi_{\mathscr{F}}\right)\right) \zeta^{4}=0
$$

since $\chi_{\mathscr{G}}+\chi \mathscr{F}=1$ on $\operatorname{supp}(\chi \mathscr{G} \mathscr{F})$ by (4.12). We thus have $\Xi_{\mathscr{G} \mathscr{F}}\left(1-\Xi_{\mathscr{G}}-\Xi_{\mathscr{F}}\right) \zeta^{4} \in$ $h \Psi_{\mathcal{T}}^{-1}\left(\mathcal{M}_{+}\right)$, so that (4.97) gives

$$
\varepsilon\left(h^{2}+\delta^{2}\right)\left\|\Xi_{\mathscr{G} \mathscr{F}} v^{r}\right\|_{1}^{2} \lesssim \varepsilon\left\|\Xi_{\mathscr{G}} v^{r}\right\|_{1}^{2}+\varepsilon\left\|\Xi_{\mathscr{F}} v^{r}\right\|_{1}^{2}+\varepsilon h^{2}\left\|v^{r}\right\|_{1}^{2} .
$$

When summing all the estimates (4.89)-(4.95) together and taking $\varepsilon$ sufficiently small, the two terms $\varepsilon\left\|\Xi_{\mathscr{G}} v^{r}\right\|_{1}^{2}, \varepsilon\left\|\Xi_{\mathscr{F}} v^{r}\right\|_{1}^{2}$ in this expression can be absorbed by the 1.h.s. of (4.89) and (4.91), respectively. This is possible since these two estimates are obtained in elliptic regions yielding better powers in $h$.

Now, if we sum all the partial estimates (4.89)-(4.95), and handle the atypical terms as explained above, we obtain

$$
\begin{array}{r}
\left\|\Xi_{\mathscr{G}} v^{r}\right\|_{1}^{2}+\mathrm{BT}\left(\Xi_{\mathscr{G}} v^{r}\right)+h\left\|\Xi_{\mathscr{G}} v^{l}\right\|_{1}^{2}+\mathrm{BT}\left(\Xi_{\mathscr{G}} v^{l}\right)+\left\|\Xi_{\mathscr{F}} v^{r}\right\|_{1}^{2}+\mathrm{BT}\left(\Xi_{\mathscr{F}} v^{r}\right)+\left\|\Xi_{\mathscr{F}} v^{l}\right\|_{1}^{2} \\
+\mathrm{BT}\left(\Xi_{\mathscr{F}} v^{l}\right)+h\left\|\Xi_{\mathscr{Z}} v^{r}\right\|_{1}^{2}+\mathrm{BT}\left(\Xi_{\mathscr{Z}} v^{r}\right)+\left\|\Xi_{\mathscr{Z}} v^{l}\right\|_{1}^{2} \\
+\mathrm{BT}\left(\Xi_{\mathscr{Z}} v^{l}\right)+\left\|\Xi_{\mathscr{E}} v^{r / l}\right\|_{1}^{2}+\mathrm{BT}\left(\Xi_{\mathscr{E}} v^{r / l}\right) \\
\lesssim \\
\operatorname{RHS}^{r}\left(v^{r}\right)+\operatorname{RHS}^{l}\left(v^{l}\right)+\left(1+\frac{\delta^{2}}{h^{2}}\right)\left\|\zeta P_{\varphi}^{r} v^{r}\right\|_{0}^{2} \\
+h^{3}\left|v^{s}\right|_{1}^{2}+h^{2}\left|D_{x_{n}} v_{\mid x_{n}=0^{+}}^{r}\right|_{0}^{2}+R_{\theta}+\frac{\delta^{2}}{h}\left|\theta_{\varphi}^{r}\right|_{0}^{2} .
\end{array}
$$


Using $\operatorname{supp}\left(v^{r / l}\right) \subset\left(\alpha_{0}, X_{0}-\alpha_{0}\right) \times S \times[0,2 \varepsilon)$ and (4.96), we can write

$$
\left\|v^{r / l}\right\|_{1} \leq\left\|\Xi_{\mathscr{G}} v^{r / l}\right\|_{1}+\left\|\Xi_{\mathscr{F}} v^{r / l}\right\|_{1}+\left\|\Xi_{\mathscr{Z}} v^{r / l}\right\|_{1}+\left\|\Xi_{\mathscr{E}} v^{r / l}\right\|_{1},
$$

together with

$$
\left|v_{\mid x_{n}=0^{+}}^{r / l}\right|_{1} \leq\left|\boldsymbol{\Xi}_{\mathscr{G}} v_{\mid x_{n}=0^{+}}^{r / l}\right|_{1}+\left|\boldsymbol{\Xi}_{\mathscr{F}} v_{\mid x_{n}=0^{+}}^{r / l}\right|_{1}+\left|\boldsymbol{\Xi}_{\mathscr{Z}} v_{\mid x_{n}=0^{+}}^{r / l}\right|_{1}+\left|\boldsymbol{\Xi}_{\mathscr{E}} v_{\mid x_{n}=0^{+}}^{r / l}\right|_{1}
$$

and

$$
\begin{aligned}
& \left|D_{x_{n}} v_{\mid x_{n}=0^{+}}^{r / l}\right|_{0} \\
& \quad \leq\left|D_{x_{n}} \Xi_{\mathscr{G}} v_{\mid x_{n}=0^{+}}^{r / l}\right|_{0}+\left|D_{x_{n}} \boldsymbol{\Xi}_{\mathscr{F}} v_{\mid x_{n}=0^{+}}^{r / l}\right|_{0}+\left|D_{x_{n}} \boldsymbol{\Xi}_{\mathscr{Z}} v_{\mid x_{n}=0^{+}}^{r / l}\right|_{0}+\left|D_{x_{n}} \boldsymbol{\Xi}_{\mathscr{E}} v_{\mid x_{n}=0^{+}}^{r / l}\right|_{0} .
\end{aligned}
$$

These three inequalities together with (4.98) give

$$
\begin{aligned}
& h\left\|v^{r / l}\right\|_{1}^{2}+h\left|v_{\mid x_{n}=0^{+}}^{r / l}\right|_{1}^{2}+h\left|D_{x_{n}} v_{\mid x_{n}=0^{+}}^{r / l}\right|_{0}^{2} \\
& \lesssim\left\|P_{\varphi}^{l} v^{l}\right\|_{0}^{2}+h^{2}\left\|v^{l}\right\|_{1}^{2}+h^{4}\left|D_{x_{n}} v_{\mid x_{n}=0^{+}}^{l}\right|_{0}^{2}+\left(1+\frac{\delta^{2}}{h^{2}}\right)\left\|\zeta P_{\varphi}^{r} v^{r}\right\|_{0}^{2}+\left\|P_{\varphi}^{r} v^{r}\right\|_{0}^{2} \\
& \quad+h^{2}\left\|v^{r}\right\|_{1}^{2}+h^{2}\left|D_{x_{n}} v_{\mid x_{n}=0^{+}}^{r}\right|_{0}^{2}+h^{3}\left|v^{s}\right|_{1}^{2}+R_{\theta}+\frac{\delta^{2}}{h}\left|\theta_{\varphi}^{r}\right|_{0}^{2} .
\end{aligned}
$$

Taking $0<h \leq h_{0}$ with $h_{0}$ sufficiently small in this expression gives

$$
\begin{aligned}
h\left\|v^{r / l}\right\|_{1}^{2}+h \mid v_{\mid x_{n}=0^{+}}^{r / l} & \left.\right|_{1} ^{2}+h\left|D_{x_{n}} v_{\mid x_{n}=0^{+}}^{r / l}\right|_{0}^{2} \\
& \lesssim\left\|P_{\varphi}^{l} v^{l}\right\|_{0}^{2}+\left\|P_{\varphi}^{r} v^{r}\right\|_{0}^{2}+\left(1+\frac{\delta^{2}}{h^{2}}\right)\left\|\zeta P_{\varphi}^{r} v^{r}\right\|_{0}^{2}+R_{\theta}+\frac{\delta^{2}}{h}\left|\theta_{\varphi}^{r}\right|_{0}^{2} .
\end{aligned}
$$

Recalling the definitions of $v^{r / l}=e^{\varphi^{r / l} / h} w^{r / l}, F_{\varphi}^{r / l}, \theta_{\varphi}^{r / l}, \Theta_{\varphi}^{s}$ (see Section 3.3 and (3.3)), and observing that

$$
\left\|e^{\varphi^{r / l} / h} D_{x_{k}} w^{r / l}\right\|_{0} \leq\left\|D_{x_{k}}\left(e^{\varphi^{r / l} / h} w^{r / l}\right)\right\|_{0}+\left\|\left(\partial_{x_{k}} \varphi^{r / l}\right) e^{\varphi^{r / l} / h} w^{r / l}\right\|_{0},
$$

and similar inequalities for the norms at the interface $\left\{x_{n}=0^{+}\right\}$, we can absorb the zeroorder terms in (3.3), which concludes the proof of Theorem 1.2.

\section{Interpolation and spectral inequalities}

\subsection{Interpolation inequality}

Here, we prove Theorem 1.4. We start by proving a local version of the interpolation inequality at the interface. In fact, the inequality we prove is local in $\left(x_{0}, x_{n}\right)$ but global on $S$. Here, we closely follow the geometrical setting of [LR10]. As in Section 3, we use local coordinates where the interface is given by $\left\{x_{n}=0\right\}$, in a small neighborhood $\left[0, X_{0}\right] \times V_{\varepsilon}$. We choose a point $z_{0} \in\left(\alpha_{1}, X_{0}-\alpha_{1}\right)$. We also pick $\alpha_{0}$ such that $0<\alpha_{0}<\alpha_{1}$ to be used when applying the Carleman estimate of Theorem 1.2. 
We define the following anisotropic distance in $\mathbb{R}^{2}$ :

$$
\operatorname{dist}_{\alpha}\left(\left(a_{0}, a_{n}\right),\left(b_{0}, b_{n}\right)\right)=\left(\alpha\left|a_{0}-b_{0}\right|^{2}+\left|a_{n}-b_{n}\right|^{2}\right)^{1 / 2}, \quad \alpha>0 .
$$

We fix $z_{n} \in \mathbb{R}_{+}^{*}$. Then, for $\left(x_{0}, x_{n}\right) \in\left[0, X_{0}\right] \times \mathbb{R}$ and $\kappa>0$, we set

$$
\psi\left(x_{0}, x_{n}\right)= \begin{cases}-\operatorname{dist}_{\alpha}\left(\left(x_{0}, x_{n}\right),\left(z_{0}, z_{n}\right)\right) & \text { if } x_{n} \geq 0, \\ -\operatorname{dist}_{\alpha}\left(\left(x_{0}, \kappa x_{n}\right),\left(z_{0}, z_{n}\right)\right) & \text { if } x_{n}<0 .\end{cases}
$$

We shall also consider $\psi$ as a function on $V_{z_{0}} \times S \times \mathbb{R}$. We note that $\psi$ is continuous across the interface $\left\{x_{n}=0\right\}$ and that

$$
\partial_{x_{n}} \psi\left(x_{0}, x_{n}\right)= \begin{cases}\left(x_{n}-z_{n}\right)\left(\psi\left(x_{0}, x_{n}\right)\right)^{-1} & \text { if } x_{n} \geq 0, \\ \kappa\left(\kappa x_{n}-z_{n}\right)\left(\psi\left(x_{0}, x_{n}\right)\right)^{-1} & \text { if } x_{n} \leq 0,\end{cases}
$$

which yields $\partial_{x_{n}} \psi_{\mid x_{n}=0^{-}}=\kappa \partial_{x_{n}} \psi_{\mid x_{n}=0^{+}}$. We also have

$$
\partial_{x_{0}} \psi\left(x_{0}, x_{n}\right)=\alpha\left(x_{0}-z_{0}\right)\left(\psi\left(x_{0}, x_{n}\right)\right)^{-1} .
$$

Let us check that the associated weight function $\varphi=e^{\lambda \psi}$ has the properties listed in Section 3.1.

According to Remark 3.3, it suffices to check that $\psi$ satisfies (3.6) and (3.7) possibly with different constants. In fact, we work in a sufficiently small neighborhood $V=V_{z_{0}} \times$ $V_{\varepsilon^{\prime}}$ of $\left\{z_{0}\right\} \times S \times\{0\}$ which does not contain $\left(z_{0}, y, z_{n}\right)$ for all $y \in S$, where $V_{z_{0}}$ is a neighborhood of $z_{0}$ in $\left(\alpha_{0}, X_{0}-\alpha_{0}\right)$ and $0<\varepsilon^{\prime}<\varepsilon$, so that $\nabla \psi$ does not vanish in $V$. First fixing $\kappa$ sufficiently small, we see that (3.6) is satisfied. Second, note that $\left|x_{0}-z_{0}\right|$ is bounded. Hence, from (5.1), we can choose the parameter $\alpha$ sufficiently small to have $\left|\partial_{x_{0}} \psi\right|$ small as compared to inf $\left|\partial_{x_{n}} \psi\right|$, so that (3.7) is satisfied. Level sets for the function $\psi$ are represented in Figure 6.

The Carleman estimate of Theorem 1.2 then follows, with the weight function $\varphi$.

We choose $0<s_{1}<s_{1}^{\prime}$ and $0<\sigma<\sigma^{\prime}$ such that

$$
\mathcal{U}^{\prime}=\left\{\left(x_{0}, y, x_{n}\right) ;\left|x_{0}-z_{0}\right|<s_{1}^{\prime}, y \in S,\left|x_{n}\right|<\sigma^{\prime}\right\} \subset V .
$$

We also set

$$
\mathcal{U}=\left\{\left(x_{0}, y, x_{n}\right) ;\left|x_{0}-z_{0}\right|<s_{1}, y \in S,\left|x_{n}\right|<\sigma\right\} \subset \mathcal{U}^{\prime} .
$$

We now choose $r_{1}<r_{1}^{\prime}<r_{2}<\psi\left(z_{0}, 0\right)<r_{2}^{\prime}<r_{3}<r_{3}^{\prime}$ such that

$$
\begin{aligned}
& \mathcal{C}_{1}=\left\{\left(x_{0}, y, x_{n}\right) \in \mathbb{R} \times S \times \mathbb{R} ; \psi\left(x_{0}, x_{n}\right)=r_{1}\right\}, \\
& \mathcal{C}_{3}^{\prime}=\left\{\left(x_{0}, y, x_{n}\right) \in \mathbb{R} \times S \times \mathbb{R} ; \psi\left(x_{0}, x_{n}\right)=r_{3}^{\prime}\right\}
\end{aligned}
$$

satisfy $\mathcal{C}_{1} \cap\left\{x_{n}<0\right\} \subset \mathcal{U}, \mathcal{C}_{1} \cap\left\{x_{n}>0\right\} \cap \mathcal{U} \neq \emptyset$, which is equivalent to

$$
\psi\left(z_{0} \pm s_{1}, 0\right)=-\left(\alpha s_{1}^{2}+z_{n}^{2}\right)^{1 / 2}<r_{1},
$$

and finally $\mathcal{C}_{3}^{\prime} \cap \mathcal{U}^{\prime} \subset\left\{x_{n} \leq \sigma\right\}$. We illustrate these choices in Figure 7. We set $R_{j}=e^{\lambda r_{j}}$, $R_{j}^{\prime}=e^{\lambda r_{j}^{\prime}}, j=1,2,3$. 


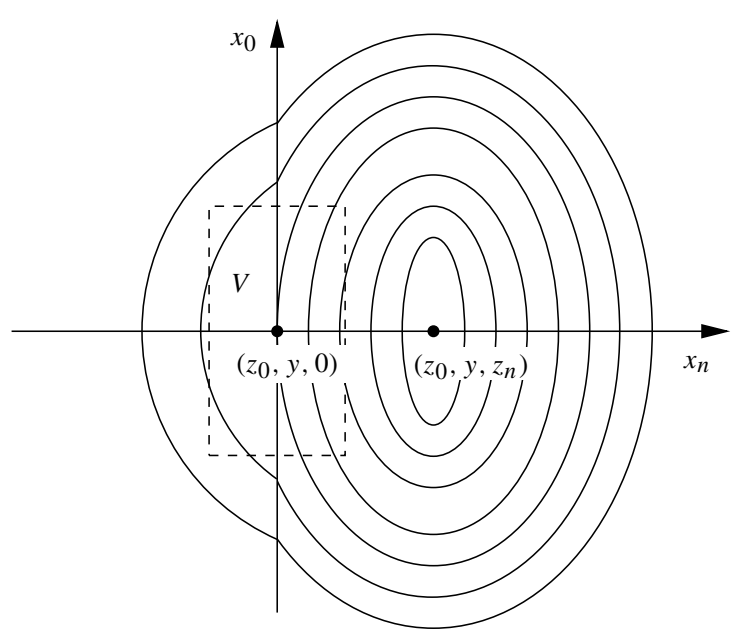

Fig. 6. Level sets for the weight functions $\psi$ and $\varphi=e^{\lambda \psi}$ in $\left(x_{0}, x_{n}\right)$ coordinates. The manifold $S \ni y$ can be represented normal to the drawing. The Carleman estimate of Theorem 1.2 can be applied in a region $V$ close to $\left\{z_{0}\right\} \times S \times\{0\}$ (represented by the dashed line).

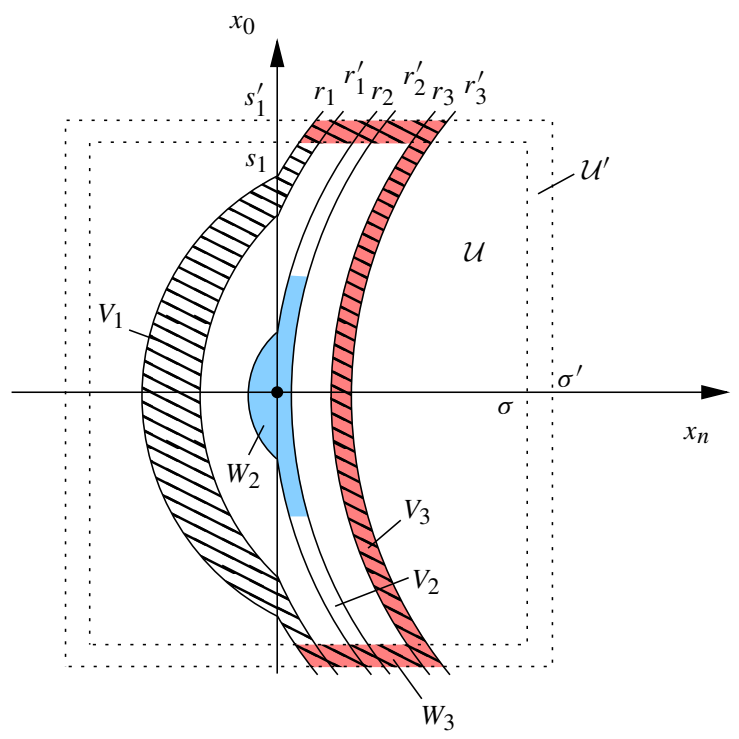

Fig. 7. Neighborhoods of the point of interest for the proof of the interpolation inequality.

Following [LR95], we introduce

$$
V_{j}:=\left\{\left(x_{0}, y, x_{n}\right) \in \mathcal{U}^{\prime} ; r_{j}<\psi\left(x_{0}, x_{n}\right)<r_{j}^{\prime}\right\}, \quad j=1,2,3,
$$


and we further set

$$
\begin{aligned}
V_{1^{\prime} \rightarrow 3} & :=\left\{\left(x_{0}, y, x_{n}\right) \in \mathcal{U} ; r_{1}^{\prime}<\psi\left(x_{0}, x_{n}\right)<r_{3}\right\}, \\
V_{1 \rightarrow 3^{\prime}}^{\prime} & :=\left\{\left(x_{0}, y, x_{n}\right) \in \mathcal{U}^{\prime} ; r_{1}<\psi\left(x_{0}, x_{n}\right)<r_{3}^{\prime}\right\}, \\
W_{3} & :=V_{3} \cup\left(V_{1 \rightarrow 3^{\prime}}^{\prime} \backslash \mathcal{U}\right) .
\end{aligned}
$$

The region $W_{3}$ is shaded and striped in Figure 7 . With the choices we have made above, $W_{3}$ is contained in $\left\{x_{n}>0\right\}$ and is finitely away from the interface $\mathbb{R}_{x_{0}} \times S=\left\{x_{n}=0\right\}$. For $s_{0} \in\left(0, s_{1}\right)$ we also choose $W_{2}=V_{2} \cap\left\{\left(x_{0}, y, x_{n}\right) ;\left|x_{0}-z_{0}\right|<s_{0}, y \in S\right\} \Subset \mathcal{U}$. The region $W_{2}$ contains $\left\{z_{0}\right\} \times S \times\{0\}$ and is shaded in Figure 7 .

Now that the geometrical context is set, we can state a local interpolation inequality in the neighborhood of $\left\{z_{0}\right\} \times S \times\{0\}$.

Lemma 5.1. For all $\delta_{0}>0$, there exist $C \geq 0$ and $\nu_{0} \in(0,1)$ such that for all $\delta \in$ $\left(0, \delta_{0}\right)$,

$$
\begin{aligned}
\|u\|_{H^{1}\left(W_{2}\right)}+\delta^{1 / 2}\left|u^{s}\right|_{H^{1}\left(W_{2} \cap\left\{x_{n}=0\right\}\right)} & \\
& \leq C\|U\|_{\mathcal{K}_{\delta}^{1}}^{1-v}\left(\|u\|_{H^{1}\left(W_{3}\right)}+\left\|\left(-\partial_{x_{0}}^{2}+A_{\delta}\right) U\right\|_{\mathcal{K}_{\delta}^{0}}\right)^{v}
\end{aligned}
$$

for all $0<v \leq v_{0}$ and $U=\left(u, u^{s}\right) \in \mathcal{K}_{\delta}^{2}$.

This inequality can be read as the "observation" of the local $\mathcal{K}_{\delta}^{1}$ norm of $U$ in the neighborhood $W_{2}$ of any strip $\left\{z_{0}\right\} \times S \times\{0\}$ by the $H^{1}$ norm of $u$ in a neighborhood away from the interface and the $\mathcal{K}_{\delta}^{0}$ norm of $\left(-\partial_{x_{0}}^{2}+A_{\delta}\right) U$.

Proof. We choose $\chi \in \mathscr{C}_{c}^{\infty}\left(\mathcal{U}^{\prime}\right)$ independent of $y \in S$ such that $\chi$ is equal to 1 on $V_{1^{\prime} \rightarrow 3}$ and vanishes outside $V_{1 \rightarrow 3^{\prime}}^{\prime}$. Then $\nabla_{x_{0}, x_{n}} \chi$ vanishes outside $V_{1 \rightarrow 3^{\prime}}^{\prime} \backslash V_{1^{\prime} \rightarrow 3}$, which is the striped region in Figure 7 .

For $U=\left(u, u^{s}\right) \in \mathcal{K}_{\delta}^{2}$, we set

$$
\left\{\begin{array}{l}
B u:=-\left(\partial_{x_{0}}^{2}+\Delta_{c}\right) u \in L^{2}\left(\left(0, X_{0}\right) \times \Omega_{1} \cup \Omega_{2}\right) \\
B^{s} U:=-\left(\partial_{x_{0}}^{2}+\Delta_{c^{s}}\right) u^{s}-\frac{1}{\delta}\left(\left(c \partial_{x_{n}} u\right)_{\mid x_{n}=0^{+}}-\left(c \partial_{x_{n}} u\right)_{\mid x_{n}=0^{-}}\right) \in L^{2}\left(\left(0, X_{0}\right) \times S\right)
\end{array}\right.
$$

and recall that $u_{\mid x_{n}=0^{-}}=u^{s}=u_{\mid x_{n}=0^{+}}$. Setting $W=\left(w, w^{s}\right)$ with $w=\chi u$ and $w^{s}=$ $\chi \mid x_{n}=0 u^{s}$, we have

$$
\begin{cases}B w=\chi B u+F, & \text { in } \mathcal{U} \\ B^{s} W=\frac{1}{\delta}\left(\delta \chi B^{s} U+\Theta\right) & \text { in } \mathcal{U} \cap S \\ w_{\mid x_{n}=0^{-}}=w^{s}=w_{\mid x_{n}=0^{+}} & \text {in } \mathcal{U} \cap S\end{cases}
$$

where

$$
\left\{\begin{array}{l}
F=\left[-\left(\partial_{x_{0}}^{2}+\Delta_{c}\right), \chi\right] u \\
\Theta=\delta\left[-\left(\partial_{x_{0}}^{2}+\Delta_{c^{s}}\right), \chi\right] u^{s}-\left(c_{\mid x_{n}=0^{+}}-c_{\mid x_{n}=0^{-}}\right) \partial_{x_{n}} \chi \mid x_{n}=0 u^{s}
\end{array}\right.
$$


Using the density result of Corollary 2.6, the Carleman estimate of Theorem 1.2 can be applied to $W=\left(w, w^{s}\right)$ :

$$
\begin{aligned}
& h\left\|e^{\varphi / h} w\right\|_{0}^{2}+h^{3}\left\|e^{\varphi / h} \nabla_{x_{0}, x} w\right\|_{0}^{2}+h\left|e^{\varphi / h} w^{s}\right|_{0}^{2}+h^{3}\left|e^{\varphi / h} \nabla_{x_{0}, S} w^{s}\right|_{0}^{2} \\
& \lesssim h^{2}\left(h^{2}+\delta^{2}\right)\left\|e^{\varphi / h} \chi B u\right\|_{0}^{2}+h^{2}\left(h^{2}+\delta^{2}\right)\left\|e^{\varphi / h} F\right\|_{0}^{2}+h^{3} \delta^{2}\left|e^{\varphi / h} \chi B^{s} U\right|_{0}^{2}+h^{3}\left|e^{\varphi / h} \Theta\right|_{0}^{2} .
\end{aligned}
$$

Note that $\Theta$ is supported in $V_{1} \cap\left\{x_{n}=0\right\}$ and in this set $e^{\varphi / h} \leq e^{R_{1}^{\prime} / h}$. Similarly, $F$ is supported in $V_{1 \rightarrow 3^{\prime}}^{\prime} \backslash V_{1^{\prime} \rightarrow 3}$ and in this set $e^{\varphi / h} \leq e^{R_{3}^{\prime} / h}$. Moreover, the operators $\left[-\left(\partial_{x_{0}}^{2}+\Delta_{c}\right), \chi\right]$ and $\left[-\left(\partial_{x_{0}}^{2}+\Delta_{c} s\right), \chi\right]$ are of order one. We thus have

$$
\begin{aligned}
\left\|e^{\varphi / h} F\right\|_{0} & \lesssim e^{R_{3}^{\prime} / h}\|u\|_{H^{1}\left(W_{3}\right)}+e^{R_{1}^{\prime} / h}\|u\|_{H^{1}\left(V_{1}\right)} \\
& \lesssim e^{R_{3}^{\prime} / h}\|u\|_{H^{1}\left(W_{3}\right)}+e^{R_{1}^{\prime} / h}\|U\|_{\mathcal{K}_{\delta}^{1}}, \\
\left|e^{\varphi / h} \Theta\right|_{0} & \lesssim e^{R_{1}^{\prime} / h}\left(\delta\left|u^{s}\right|_{H^{1}\left(V_{1} \cap\left\{x_{n}=0\right\}\right)}+\left|u^{s}\right|_{L^{2}\left(V_{1} \cap\left\{x_{n}=0\right\}\right)}\right) .
\end{aligned}
$$

Using the trace formula together with $\delta \leq \delta^{1 / 2} \delta_{0}^{1 / 2}$ in this last inequality, we obtain

$$
\left|e^{\varphi / h} \Theta\right|_{0} \lesssim e^{R_{1}^{\prime} / h}\left(\delta^{1 / 2}\left|u^{s}\right|_{H^{1}\left(\left(0, X_{0}\right) \times S\right)}+\|u\|_{H^{1}\left(\mathcal{U}^{\prime}\right)}\right) \lesssim e^{R_{1}^{\prime} / h}\|U\|_{\mathcal{K}_{\delta}^{1}}
$$

Moreover,

$$
\begin{aligned}
\left\|e^{\varphi / h} \chi B u\right\|_{0} & \lesssim e^{R_{3}^{\prime} / h}\|B u\|_{L^{2}\left(\mathcal{U}^{\prime}\right)} \lesssim e^{R_{3}^{\prime} / h}\left\|\left(-\partial_{x_{0}}^{2}+A_{\delta}\right) U\right\|_{\mathcal{K}_{\delta}^{0}} \\
\delta^{1 / 2}\left|e^{\varphi / h} \chi B^{s} U\right|_{0} & \lesssim \delta^{1 / 2} e^{R_{3}^{\prime} / h}\left|B^{s} U\right|_{L^{2}\left(\mathcal{U}^{\prime} \cap\left\{x_{n}=0\right\}\right)} \lesssim e^{R_{3}^{\prime} / h}\left\|\left(-\partial_{x_{0}}^{2}+A_{\delta}\right) U\right\|_{\mathcal{K}_{\delta}^{0}}
\end{aligned}
$$

Concerning the 1.h.s. of (5.3), we have $e^{\varphi / h} \geq e^{R_{2} / h}$ and $\chi=1$ on $W_{2}$, so that, using $\delta \leq \delta_{0}$

$$
\begin{aligned}
h\left\|e^{\varphi / h} w\right\|_{0}^{2}+h^{3}\left\|e^{\varphi / h} \nabla_{x_{0}, y, x_{n}} w\right\|_{0}^{2}+h\left|e^{\varphi / h} w^{s}\right|_{0}^{2}+h^{3}\left|e^{\varphi / h} \nabla_{x_{0}, y} w^{s}\right|_{0}^{2} \\
\\
\gtrsim h^{3} e^{2 R_{2} / h}\|u\|_{H^{1}\left(W_{2}\right)}^{2}+h^{3} \delta e^{2 R_{2} / h}\left|u^{s}\right|_{H^{1}\left(W_{2} \cap\left\{x_{n}=0\right\}\right)}^{2} .
\end{aligned}
$$

Using (5.4)-(5.8) in (5.3), we thus obtain

$$
\begin{aligned}
h^{3 / 2} e^{R_{2} / h}\left(\|u\|_{H^{1}\left(W_{2}\right)}+\delta^{1 / 2}\left|u^{s}\right|_{H^{1}\left(W_{2} \cap\left\{x_{n}=0\right\}\right)}\right) & \\
& \lesssim h\left(e^{R_{1}^{\prime} / h}\|U\|_{\mathcal{K}_{\delta}^{1}}+e^{R_{3}^{\prime} / h}\left(\left\|\left(-\partial_{x_{0}}^{2}+A_{\delta}\right) U\right\|_{\mathcal{K}_{\delta}^{0}}+\|u\|_{H^{1}\left(W_{3}\right)}\right)\right) .
\end{aligned}
$$

Fixing some $\tilde{R}_{2} \in\left(R_{1}^{\prime}, R_{2}\right)$, we have $h^{1 / 2} e^{R_{2} / h} \gtrsim e^{\tilde{R}_{2} / h}$ for all $0<h<h_{0}$. Thus, (5.9) becomes

$$
\begin{aligned}
e^{\tilde{R}_{2} / h}\left(\|u\|_{H^{1}\left(W_{2}\right)}+\right. & \left.\delta^{1 / 2}\left|u^{s}\right|_{H^{1}\left(W_{2} \cap\left\{x_{n}=0\right\}\right)}\right) \\
& \lesssim e^{R_{1}^{\prime} / h}\|U\|_{\mathcal{K}_{\delta}^{1}}+e^{R_{3}^{\prime} / h}\left(\left\|\left(-\partial_{x_{0}}^{2}+A_{\delta}\right) U\right\|_{\mathcal{K}_{\delta}^{0}}+\|u\|_{H^{1}\left(W_{3}\right)}\right) .
\end{aligned}
$$

Finally, optimizing with respect to $h$ as in [Rob95] we obtain the desired local interpolation inequality. 
Away from the interface, the $\mathcal{K}_{\delta}^{s}$ norms, $s=0,1$, coincide with the usual $H^{s}$ norm, and local interpolation inequalities similar to (5.2) are proven in [LR95, Lemme 3, p. 352]. Now that we have obtained the interpolation inequality (5.2) at the interface, we can apply the procedure described in [LR95, pp. 353-356] (propagation of smallness) and prove the desired global interpolation inequality (1.13). See [LZ98, proof of Theorem 3] to obtain the term $\left\|\partial_{x_{0}} u(0, x)\right\|_{L^{2}(\omega)}$ on the r.h.s. of (1.13). This concludes the proof of Theorem 1.4.

\subsection{Spectral inequality}

From the interpolation inequality proven in Theorem 1.4, we now deduce the uniform spectral inequality of Theorem 1.5. Recall that $\mathscr{E}_{\delta, j}=\left(e_{\delta, j}, e_{\delta, j}^{s}\right), j \in \mathbb{N}$, denotes a Hilbert basis of $\mathcal{H}_{\delta}^{0}$ composed of eigenfunctions of the operator $A_{\delta}$ associated with the positive eigenvalues $\mu_{\delta, j} \in \mathbb{R}, j \in \mathbb{N}$, sorted in an increasing sequence. We denote by $\Pi_{\delta, \mu}$ the spectral projector over the eigenfunctions associated with eigenvalues lower than $\mu$, i.e.,

$$
\Pi_{\delta, \mu} Y=\sum_{\mu_{\delta, j} \leq \mu}\left(Y, \mathscr{E}_{\delta, j}\right)_{\mathcal{H}_{\delta}^{0}} \mathscr{E}_{\delta, j}, \quad Y \in \mathcal{H}_{\delta}^{0}
$$

The proof of Theorem 1.5 is classical. Yet, we have to make sure that all the constants involved are independent of the parameter $\delta$.

First we take some $Y_{\delta}=\left(y_{\delta}, y_{\delta}^{s}\right) \in \Pi_{\delta, \mu} \mathcal{H}_{\delta}^{0}$, and apply the interpolation inequality (1.13) of Theorem 1.4 to

$$
U_{\delta}=\left(u_{\delta}, u_{\delta}^{s}\right)=A_{\delta}^{-1 / 2} \sinh \left(x_{0} A_{\delta}^{1 / 2}\right) Y_{\delta}
$$

defined by using the classical functional calculus for self-adjoint operators. ${ }^{5}$

We notice that $\left(-\partial_{x_{0}}^{2}+A_{\delta}\right) U_{\delta}=0, U_{\delta}(0, x)=0$ and $\left\|\partial_{x_{0}} u_{\delta}(0, x)\right\|_{L^{2}(\omega)}=\left\|y_{\delta}\right\|_{L^{2}(\omega)}$. Concerning the 1.h.s. of the interpolation inequality (1.13), we have

$$
\begin{aligned}
\left\|U_{\delta}\right\|_{\mathcal{K}_{\delta}^{1}\left(\alpha_{1}\right)}^{2} & \geq\left\|U_{\delta}\right\|_{\mathcal{K}_{\delta}^{0}\left(\alpha_{1}\right)}^{2}=\left\|U_{\delta}\right\|_{L^{2}\left(\alpha_{1}, X_{0}-\alpha_{1} ; \mathcal{H}_{\delta}^{0}\right)}^{2} \\
& =\int_{\alpha_{1}}^{X_{0}-\alpha_{1}}\left\|A_{\delta}^{-1 / 2} \sinh \left(x_{0} A_{\delta}^{1 / 2}\right) Y_{\delta}\right\|_{\mathcal{H}_{\delta}^{0}}^{2} d x_{0} \\
& \geq \int_{\alpha_{1}}^{X_{0}-\alpha_{1}}\left\|\left[A_{\delta}^{-1 / 2} \sinh \left(x_{0} A_{\delta}^{1 / 2}\right) \Pi_{\delta, \mu}\right]^{-1}\right\|_{\mathcal{L}_{\left(\mathcal{H}_{\delta}^{0}\right)}^{-2}}^{X_{0}}\left\|Y_{\delta}\right\|_{\mathcal{H}_{\delta}^{0}}^{2} d x_{0} \\
& \geq \int_{\alpha_{1}}^{X_{0}-\alpha_{1}} x_{0}^{2} d x_{0}\left\|Y_{\delta}\right\|_{\mathcal{H}_{\delta}^{0}}^{2} \geq C\left(X_{0}, \alpha_{1}\right)\left\|Y_{\delta}\right\|_{\mathcal{H}_{\delta}^{0}}^{2},
\end{aligned}
$$

\footnotetext{
5 Note that if $A_{\delta}$ is not invertible, i.e. $0 \in \operatorname{Sp}\left(A_{\delta}\right)$ (this occurs if $\Omega$ has no boundary), the following analysis can be done with $A_{\delta}+$ Id in place of $A_{\delta}$. Theorems 1.2 and 1.4 remain valid for this operator. The spectral inequality proven for $A_{\delta}+$ Id implies the same inequality for $A_{\delta}$.
} 
since $t^{-1 / 2} \sinh \left(x_{0} t^{1 / 2}\right) \geq x_{0}$ for $t>0$. Now, concerning the r.h.s. of (1.13) we have

$$
\begin{aligned}
\left\|U_{\delta}\right\|_{\mathcal{K}_{\delta}^{1}}^{2} & =\left\|U_{\delta}\right\|_{\mathcal{K}_{\delta}^{0}}^{2}+\left\|A_{\delta}^{1 / 2} U_{\delta}\right\|_{\mathcal{K}_{\delta}^{0}}^{2}+\left\|\partial_{x_{0}} U_{\delta}\right\|_{\mathcal{K}_{\delta}^{0}}^{2} \\
& =\int_{0}^{X_{0}}\left(\left\|U_{\delta}\right\|_{\mathcal{H}_{\delta}^{0}}^{2}+\left\|A_{\delta}^{1 / 2} U_{\delta}\right\|_{\mathcal{H}_{\delta}^{0}}^{2}+\left\|\partial_{x_{0}} U_{\delta}\right\|_{\mathcal{H}_{\delta}^{0}}^{2}\right) d x_{0} .
\end{aligned}
$$

Let us estimate the three terms in this expression. First, we have

$$
\begin{aligned}
\int_{0}^{X_{0}}\left\|U_{\delta}\right\|_{\mathcal{H}_{\delta}^{0}}^{2} d x_{0} & \leq \int_{0}^{X_{0}}\left\|A_{\delta}^{-1 / 2} \sinh \left(x_{0} A_{\delta}^{1 / 2}\right) \Pi_{\delta, \mu}\right\|_{\mathcal{L}\left(\mathcal{H}_{\delta}^{0}\right)}^{2}\left\|Y_{\delta}\right\|_{\mathcal{H}_{\delta}^{0}}^{2} d x_{0} \\
& \leq \int_{0}^{X_{0}}\left(x_{0} e^{x_{0} \sqrt{\mu}}\right)^{2} d x_{0}\left\|Y_{\delta}\right\|_{\mathcal{H}_{\delta}^{0}}^{2} \leq X_{0}^{3} e^{2 X_{0} \sqrt{\mu}}\left\|Y_{\delta}\right\|_{\mathcal{H}_{\delta}^{0}}^{2},
\end{aligned}
$$

since $t^{-1 / 2} \sinh \left(x_{0} t^{1 / 2}\right) \leq x_{0} e^{x_{0} \sqrt{\mu}}$ for $0 \leq t \leq \mu$. Second, we have

$$
\begin{aligned}
\int_{0}^{X_{0}}\left\|A_{\delta}^{1 / 2} U_{\delta}\right\|_{\mathcal{H}_{\delta}^{0}}^{2} d x_{0} & \leq \int_{0}^{X_{0}}\left\|\sinh \left(x_{0} A_{\delta}^{1 / 2}\right) \Pi_{\delta, \mu}\right\|_{\mathcal{L}\left(\mathcal{H}_{\delta}^{0}\right)}^{2}\left\|Y_{\delta}\right\|_{\mathcal{H}_{\delta}^{0}}^{2} d x_{0} \\
& \leq \int_{0}^{X_{0}} e^{2 x_{0} \sqrt{\mu}} d x_{0}\left\|Y_{\delta}\right\|_{\mathcal{H}_{\delta}^{0}}^{2} \leq X_{0} e^{2 X_{0} \sqrt{\mu}}\left\|Y_{\delta}\right\|_{\mathcal{H}_{\delta}^{0}}^{2},
\end{aligned}
$$

together with

$$
\begin{aligned}
\int_{0}^{X_{0}}\left\|\partial_{x_{0}} U_{\delta}\right\|_{\mathcal{H}_{\delta}^{0}}^{2} d x_{0} & =\int_{0}^{X_{0}}\left\|\cosh \left(x_{0} A_{\delta}^{1 / 2}\right) Y_{\delta}\right\|_{\mathcal{H}_{\delta}^{0}}^{2} d x_{0} \leq \int_{0}^{X_{0}} e^{2 x_{0} \sqrt{\mu}} d x_{0}\left\|Y_{\delta}\right\|_{\mathcal{H}_{\delta}^{0}}^{2} \\
& \leq X_{0} e^{2 X_{0} \sqrt{\mu}}\left\|Y_{\delta}\right\|_{\mathcal{H}_{\delta}^{0}}^{2}
\end{aligned}
$$

By using the last three estimates in (5.11), together with (5.10), the interpolation inequality (1.13) yields

$$
\left\|Y_{\delta}\right\|_{\mathcal{H}_{\delta}^{0}} \leq C\left(X_{0}, \alpha_{1}\right)\left(e^{X_{0} \sqrt{\mu}}\left\|Y_{\delta}\right\|_{\mathcal{H}_{\delta}^{0}}\right)^{1-\nu_{0}}\left\|y_{\delta}\right\|_{L^{2}(\omega)}^{\nu_{0}} .
$$

Finally, for $\delta_{0}>0$, there exists $C>0$ such that for all $0<\delta \leq \delta_{0}$ and $\mu \in \mathbb{R}$, we have

$$
\left\|Y_{\delta}\right\|_{\mathcal{H}_{\delta}^{0}} \leq C e^{X_{0} \frac{1-v_{0}}{v_{0}} \sqrt{\mu}}\left\|y_{\delta}\right\|_{L^{2}(\omega)}, \quad Y_{\delta}=\left(y_{\delta}, y_{\delta}^{s}\right) \in \Pi_{\delta, \mu} \mathcal{H}_{\delta}^{0} .
$$

This concludes the proof of Theorem 1.5.

\section{Appendix A. Derivation of the model}

Here, we (formally) derive the model (1.4) studied in the main part of this article. We use the notation of the beginning of Section 3. In a small neighborhood of the interface $S$ we use normal geodesic coordinates

$$
\mathrm{F}: S \times[-2 \varepsilon, 2 \varepsilon] \rightarrow V_{\varepsilon}, \quad\left(y, x_{n}\right) \mapsto \mathrm{F}\left(y, x_{n}\right) .
$$




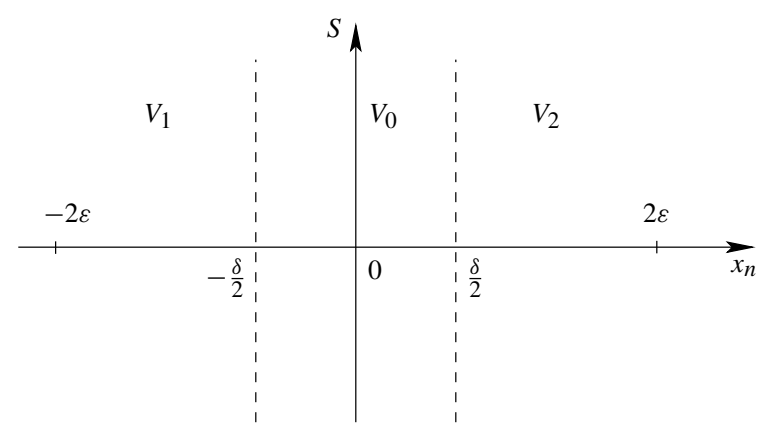

Fig. 8. Local geometry of a three-layer model near the interface $S=\left\{x_{n}=0\right\}$. The inner layer, $V_{0}$, shrinks to zero as $\delta$ goes to zero.

In such coordinates the metric reads

$$
g=\left(\begin{array}{cc}
g_{\mid T(S)} & 0 \\
0 & 1
\end{array}\right)
$$

and the elliptic operators we consider, $-\operatorname{div}_{g} c \nabla_{g}$, take the form $-\partial_{x_{n}} c \partial_{x_{n}}-\operatorname{div}^{s} c \nabla^{s}$. The interface $S$ is given by $\left\{x_{n}=0\right\}$.

Let $\delta \in(0,4 \varepsilon)$. We consider three regions in $V_{\varepsilon}$ as represented in Figure 8:

$$
V_{1}=\left\{-2 \varepsilon \leq x_{n} \leq-\delta / 2\right\}, \quad V_{0}=\left\{-\delta / 2 \leq x_{n} \leq \delta / 2\right\}, \quad V_{2}=\left\{\delta / 2 \leq x_{n} \leq 2 \varepsilon\right\} .
$$

With three coefficients $c^{0}, c^{1}, c^{2}$ we consider the following parabolic problem:

$$
\partial_{t} z^{j}-\operatorname{div}_{g}\left(c^{j} \nabla_{g} z^{j}\right)=f^{j} \quad \text { in }(0, T) \times V_{j}, \quad j=1,0,2,
$$

along with the natural transmission conditions at $x_{n}=\delta / 2$ and $x_{n}=-\delta / 2$, given by the continuity of the solution and the continuity of the flux:

$$
z_{\mid x_{n}=-\delta / 2}^{1}=z_{\mid x_{n}=-\delta / 2}^{0}, \quad z_{\mid x_{n}=\delta / 2}^{0}=z_{\mid x_{n}=\delta / 2}^{2},
$$

and

$$
\left(c^{1} \partial_{x_{n}} z^{1}\right)_{\mid x_{n}=-\delta / 2}=\left(c^{0} \partial_{x_{n}} z^{0}\right)_{\mid x_{n}=-\delta / 2}, \quad\left(c^{0} \partial_{x_{n}} z^{0}\right)_{\mid x_{n}=\delta / 2}=\left(c^{2} \partial_{x_{n}} z^{2}\right)_{\mid x_{n}=\delta / 2} .
$$

We now wish to describe the present three-region model as the thickness $\delta$ of the inner region, $V_{0}$, becomes asymptotically small. This implies some approximation. Resulting approximate models can be very useful in practice as one is in need of effective models.

We introduce the mean values of $z^{0}$ and $f^{0}$ in the normal direction $x_{n}$,

$$
z^{s}(y):=\frac{1}{\delta} \int_{-\delta / 2}^{\delta / 2} z^{0}\left(y, x_{n}\right) d x_{n} \quad \text { and } \quad f^{s}(y):=\frac{1}{\delta} \int_{-\delta / 2}^{\delta / 2} f^{0}\left(y, x_{n}\right) d x_{n}, \quad y \in S .
$$

Keeping in mind that $\delta$ is meant to be asymptotically small, we first make the following approximation.

Assumption A.1. The diffusion coefficient $c^{0}$ does not depend on the normal variable $x_{n}$. We $\operatorname{set}^{s}(y)=c^{0}\left(y, x_{n}\right)$. 
Under this assumption, using the transmission conditions (A.3), we have

$$
\begin{aligned}
f^{s}(y) & =\frac{1}{\delta} \int_{-\delta / 2}^{\delta / 2}\left(\partial_{t} z^{0}-\operatorname{div}_{g}\left(c^{0} \nabla_{g} z^{0}\right)\right) d x_{n} \\
& =\partial_{t} z^{s}-\operatorname{div}^{s}\left(c^{s} \nabla^{s} z^{s}\right)-\frac{1}{\delta} \int_{-\delta / 2}^{\delta / 2} \partial_{x_{n}} c^{0} \partial_{x_{n}} z^{0} d x_{n} \\
& =\partial_{t} z^{s}-\operatorname{div}^{s}\left(c^{s} \nabla^{s} z^{s}\right)-\frac{1}{\delta}\left(\left(c^{0} \partial_{x_{n}} z^{0}\right)_{\mid x_{n}=\delta / 2}-\left(c^{0} \partial_{x_{n}} z^{0}\right)_{\mid x_{n}=-\delta / 2}\right) \\
& =\partial_{t} z^{s}-\operatorname{div}^{s}\left(c^{s} \nabla^{s} z^{s}\right)-\frac{1}{\delta}\left(\left(c^{2} \partial_{x_{n}} z^{2}\right)_{\mid x_{n}=\delta / 2}-\left(c^{1} \partial_{x_{n}} z^{1}\right)_{\mid x_{n}=-\delta / 2}\right)
\end{aligned}
$$

This provides a first transmission condition between $z^{1}$ and $z^{2}$ that involves the function $z^{s}$. For the problem to be closed, we need two additional transmission conditions.

We begin with a first-order approximation of the system. Yet we show that it cannot be used for the purpose of modeling controllability properties of the original system. We then lower the degree of our approximations and obtain the model studied in the main part of this article.

\section{A.1. A first-order model}

Using the transmission conditions (A.2)-(A.3) we write

$$
\begin{gathered}
z^{2}(y, \delta / 2)-z^{1}(y,-\delta / 2)=z^{0}(y, \delta / 2)-z^{0}(y,-\delta / 2)=\int_{-\delta / 2}^{\delta / 2} \partial_{x_{n}} z^{0}\left(y, x_{n}\right) d x_{n} \\
=\left[x_{n} \partial_{x_{n}} z^{0}\left(y, x_{n}\right)\right]_{-\delta / 2}^{\delta / 2}+R_{1}=\delta / 2\left(\partial_{x_{n}} z^{0}(y, \delta / 2)+\partial_{x_{n}} z^{0}(y,-\delta / 2)\right)+R_{1} \\
=\frac{\delta}{2 c^{s}(y)}\left(c^{2}(y, \delta / 2) \partial_{x_{n}} z^{2}(y, \delta / 2)+c^{1}(y,-\delta / 2) \partial_{x_{n}} z^{1}(y,-\delta / 2)\right)+R_{1},
\end{gathered}
$$

with $R_{1}=-\int_{-\delta / 2}^{\delta / 2} x_{n} \partial_{x_{n}}^{2} z^{0}\left(y, x_{n}\right) d x_{n}$.

A second set of transmission conditions is needed. With two integrations by parts we write

$$
\begin{aligned}
z^{s}(y)= & \frac{1}{\delta} \int_{-\delta / 2}^{\delta / 2} z^{0}\left(y, x_{n}\right) d x_{n} \\
= & \frac{1}{\delta}\left[x_{n} z^{0}\left(y, x_{n}\right)\right]_{-\delta / 2}^{\delta / 2}-\frac{1}{\delta}\left[\frac{x_{n}^{2}}{2} \partial_{x_{n}} z^{0}\left(y, x_{n}\right)\right]_{-\delta / 2}^{\delta / 2}+\frac{1}{\delta} \int_{-\delta / 2}^{\delta / 2} \frac{x_{n}^{2}}{2} \partial_{x_{n}}^{2} z^{0}\left(y, x_{n}\right) d x_{n} \\
= & \frac{1}{2}\left(z^{0}(y, \delta / 2)+z^{0}(y,-\delta / 2)\right)-\frac{\delta}{8}\left(\partial_{x_{n}} z^{0}(y, \delta / 2)-\partial_{x_{n}} z^{0}(y,-\delta / 2)\right) \\
& +\frac{1}{\delta} \int_{-\delta / 2}^{\delta / 2} \frac{x_{n}^{2}}{2} \partial_{x_{n}}^{2} z^{0}\left(y, x_{n}\right) d x_{n} \\
= & \frac{1}{2}\left(z^{2}(y, \delta / 2)+z^{1}(y,-\delta / 2)\right)+R_{2}
\end{aligned}
$$


with

$$
R_{2}=-\frac{\delta}{8}\left(\partial_{x_{n}} z^{0}(y, \delta / 2)-\partial_{x_{n}} z^{0}(y,-\delta / 2)\right)+\frac{1}{\delta} \int_{-\delta / 2}^{\delta / 2} \frac{x_{n}^{2}}{2} \partial_{x_{n}}^{2} z^{0}\left(y, x_{n}\right) d x_{n}
$$

We now make the following assumption on the variations of $z^{0}$ with respect to $x_{n}$.

Assumption A.2. We have $\left|\partial_{x_{n}}^{2} z^{0}\left(y, x_{n}\right)\right| \leq C$ uniformly in $\delta$ (and $x_{n}$, and $y \in S$ ).

We then find that $R_{1}=O\left(\delta^{2}\right)$. Observe that

$$
\partial_{x_{n}} z^{0}(y, \delta / 2)-\partial_{x_{n}} z^{0}(y,-\delta / 2)=\int_{-\delta / 2}^{\delta / 2} \partial_{x_{n}}^{2} z^{0}\left(y, x_{n}\right) d x_{n}=O(\delta) .
$$

It follows that $R_{2}=O\left(\delta^{2}\right)$.

To first order in $\delta$ we thus obtain

$$
\left\{\begin{array}{l}
z^{2}(y, \delta / 2)-z^{1}(y,-\delta / 2) \\
\quad=\frac{\delta}{2 c^{s}(y)}\left(c^{2}(y, \delta / 2) \partial_{x_{n}} z^{2}(y, \delta / 2)+c^{1}(y,-\delta / 2) \partial_{x_{n}} z^{1}(y,-\delta / 2)\right) \\
z^{s}(y)=\frac{1}{2}\left(z^{2}(y, \delta / 2)+z^{1}(y,-\delta / 2)\right) .
\end{array}\right.
$$

As $\delta$ is small we assume that $z^{1}$ and $z^{2}$ are defined on $\left\{x_{n}<0\right\}$ and $\left\{x_{n}>0\right\}$ respectively. We thus write $z_{\mid x_{n}=0^{+}}^{2}$ and $c^{2} \partial_{x_{n}} z_{\mid x_{n}=0^{+}}^{2}$ in place of $z^{2}(y, \delta / 2)$ and $\left(c^{2} \partial_{x_{n}} z^{2}\right)(y, \delta / 2)$ and similarly $z_{\mid x_{n}=0^{-}}^{1}$ and $c^{1} \partial_{x_{n}} z_{\mid x_{n}=0^{-}}^{1}$ in place of $z^{1}(y,-\delta / 2)$ and $\left(c^{2} \partial_{x_{n}} z^{2}\right)(y,-\delta / 2)$. We obtain the following model:

$$
\partial_{t} z^{j}-\operatorname{div}_{g} c^{j} \nabla_{g} z^{j}=f^{j} \quad \text { in }(0, T) \times \Omega_{j}, j=1,2,
$$

and

$$
\left\{\begin{array}{l}
\partial_{t} z^{s}-\operatorname{div}^{s}\left(c^{s} \nabla^{s} z^{s}\right)=f^{s}+\frac{1}{\delta}\left(\left(c^{2} \partial_{x_{n}} z^{2}\right)_{\mid x_{n}=0^{+}}-\left(c^{1} \partial_{x_{n}} z^{1}\right)_{\mid x_{n}=0^{-}}\right) \\
z^{s}=\frac{1}{2}\left(z_{\mid x_{n}=0^{+}}^{2}+z_{\mid x_{n}=0^{-}}^{1}\right) \\
z_{\mid x_{n}=0^{+}}^{2}-z_{\mid x_{n}=0^{-}}^{1}=\frac{\delta}{2 c^{s}}\left(\left(c^{2} \partial_{x_{n}} z^{2}\right)_{\mid x_{n}=0^{+}}+\left(c^{1} \partial_{x_{n}} z^{1}\right)_{\mid x_{n}=0^{-}}\right)
\end{array}\right.
$$

in $(0, T) \times S$.

For the study of the controllability of such a parabolic model we wish to investigate the unique continuation properties of the associated elliptic problem:

$$
-\operatorname{div}_{g} c^{j} \nabla_{g} z^{j}=f^{j} \quad \text { in }(0, T) \times \Omega_{j}, j=1,2,
$$

and

$$
\left\{\begin{array}{l}
-\operatorname{div}^{s}\left(c^{s} \nabla^{s} z^{s}\right)=f^{s}+\frac{1}{\delta}\left(\left(c^{2} \partial_{x_{n}} z^{2}\right)_{\mid x_{n}=0^{+}}-\left(c^{1} \partial_{x_{n}} z^{1}\right)_{\mid x_{n}=0^{-}}\right), \\
z^{s}=\frac{1}{2}\left(z_{\mid x_{n}=0^{+}}^{2}+z_{\mid x_{n}=0^{-}}^{1}\right) \\
z_{\mid x_{n}=0^{+}}^{2}-z_{\mid x_{n}=0^{-}}^{1}=\frac{\delta}{2 c^{s}}\left(\left(c^{2} \partial_{x_{n}} z^{2}\right)_{\mid x_{n}=0^{+}}+\left(c^{1} \partial_{x_{n}} z^{1}\right)_{\mid x_{n}=0^{-}}\right),
\end{array}\right.
$$

in $(0, T) \times S$. 
Note that unique continuation holds for the original problem. This is an important property that we wish to see preserved in this approximation process. Here, we show that there are instances for which eigenfunctions of the elliptic operator in the approximate model (A.8)-(A.9) vanish on one side of the interface. These eigenmodes are then invisible when considering the observability of the parabolic system (A.6)-(A.7), ruining any hope of controllability. This is similar to the situation described in Section 1.3.3.

Let us consider the following two-dimensional example: $\Omega=\mathbb{R} /(2 \pi \mathbb{Z}) \times(-\pi, \pi)$ is the cylinder endowed with a flat metric. For consistency with the notation of Section 3 we use $\left(y, x_{n}\right)$ as the coordinates in $\Omega$, with periodic conditions in $y$. We define the interface as $S=\left\{x_{n}=0\right\}=\mathbb{R} /(2 \pi \mathbb{Z}) \times\{0\}$, so that $\Omega_{1}=\left\{x_{n}<0\right\}$ and $\Omega_{2}=\left\{x_{n}>0\right\}$.

Proposition A.3. Let $c^{s}$ and $c^{1}$ be constant functions such that $c^{s}=r c^{1}$ with $r>1$. For any $\delta_{0}>0$, there exist $0<\delta \leq \delta_{0}, e^{1} \in \mathscr{C}^{\infty}\left(\Omega_{1}\right), e^{s} \in \mathscr{C}^{\infty}(S), \lambda>0$ such that

$$
-\operatorname{div}_{g} c^{1} \nabla_{g} e^{1}=\lambda e^{1} \quad \text { in } \Omega_{1}, \quad-\operatorname{div}^{s}\left(c^{s} \nabla^{s} e^{s}\right)+\frac{1}{\delta}\left(c^{1} \partial_{x_{n}} e^{1}\right)_{\mid x_{n}=0^{-}}=\lambda e^{s} \quad \text { in } S,
$$

and

$$
e^{s}=\frac{1}{2} e_{\mid x_{n}=0^{-}}^{1}, \quad-e_{\mid x_{n}=0^{-}}^{1}=\frac{\delta}{2 c^{s}}\left(c^{1} \partial_{x_{n}} e^{1}\right)_{\mid x_{n}=0^{-}}, \quad \text { in } S,
$$

and $e_{\mid x_{n}=-\pi}^{1}=0$. Hence $\left(e^{1}, e^{s}, 0\right)$ is an eigenfunction of the elliptic operator in (A.8)(A.9) associated with the eigenvalue $\lambda$, for Dirichlet boundary conditions (in $x_{n}$ ).

Proof. We choose $k \in \mathbb{N}$ such that $(r-1) k>1$. For $\mu \in(0,1)$ we set

$$
g(\mu)=\left(\frac{1}{r\left((r-1) k^{2}-\mu^{2}\right)}\right)^{1 / 2} \frac{\mu \cos (\mu \pi)}{\sin (\mu \pi)} .
$$

As $g$ vanishes for $\mu=1 / 2$ and $\lim _{\mu \rightarrow 1^{-}} g(\mu)=-\infty$, there exists $\mu_{0} \in(1 / 2,1)$ such that $g\left(\mu_{0}\right)=-1$. We then set

$$
\delta=2\left(\frac{r}{\left((r-1) k^{2}-\mu_{0}^{2}\right)}\right)^{1 / 2}, \quad \alpha=\frac{2}{\sin \left(\mu_{0} \pi\right)} .
$$

For any given $\delta_{0}$ we can have $0<\delta \leq \delta_{0}$ by choosing $k$ sufficiently large. We have

$$
\frac{\delta \mu_{0} \cos \left(\mu_{0} \pi\right)}{2 r \sin \left(\mu_{0} \pi\right)}=-1
$$

We now set

$$
e^{s}(y)=e^{i k y}, \quad e^{1}\left(y, x_{n}\right)=\alpha \sin \left(\mu_{0}\left(x_{n}+\pi\right)\right) e^{s}(y), \quad-\pi \leq x_{n} \leq 0 .
$$

We have $e_{\mid x_{n}=-\pi}^{1}=0$. Hence the Dirichlet boundary condition is satisfied at $x_{n}=-\pi$. 
We have $-c^{1}\left(\partial_{y}^{2}+\partial_{x_{n}}^{2}\right) e^{1}=\lambda e^{1}$ with $\lambda=c^{1}\left(k^{2}+\mu_{0}^{2}\right)$. Observing that $\partial_{x_{n}} e_{\mid x_{n}=0^{-}}^{1}=$ $\alpha \mu_{0} \cos \left(\mu_{0} \pi\right) e^{s}$ we find

$$
\begin{aligned}
-c^{s} \partial_{y}^{2} e^{s}+\frac{1}{\delta} c \partial_{x_{n}} e_{\mid x_{n}=0^{-}}^{1} & =c^{1}\left(r k^{2}+\frac{\alpha \mu_{0}}{\delta} \cos \left(\mu_{0} \pi\right)\right) e^{s} \\
& =c^{1}\left(r k^{2}+\frac{2 \mu_{0}}{\delta \sin \left(\mu_{0} \pi\right)} \cos \left(\mu_{0} \pi\right)\right) e^{s}=c^{1}\left(r k^{2}-\frac{4 r}{\delta^{2}}\right) e^{s} \\
& =c^{1}\left(r k^{2}-\left((r-1) k^{2}-\mu_{0}\right)\right) e^{s}=\lambda e^{s},
\end{aligned}
$$

by (A.12) and the value we have assigned to $\delta$. We have thus obtained (A.10).

We now compute, using (A.12) and the value we have assigned to $\alpha$,

$$
\frac{1}{2} e_{\mid x_{n}=0^{-}}^{1}=\frac{\alpha}{2} \sin \left(\mu_{0} \pi\right) e^{s}=e^{s} .
$$

Using (A.12) we also compute

$$
e_{\mid x_{n}=0^{-}}^{1}+\frac{\delta}{2 c^{s}}\left(c^{1} \partial_{x_{n}} e^{1}\right)_{\mid x_{n}=0^{-}}=\alpha \sin \left(\mu_{0} \pi\right)\left(1+\frac{\delta}{2 r} \mu_{0} \frac{\cos \left(\mu_{0} \pi\right)}{\sin \left(\mu_{0} \pi\right)}\right) e^{s}=0 .
$$

We have thus obtained (A.11).

\section{A.2. A zero-order model}

The lack of unique continuation of the previous (elliptic) model makes us consider a simpler model. We make a lower-order approximation and we show how to formally obtain the model studied in the main text of this article.

Neglecting the first-order terms in $\delta$ in (A.5) we find

$$
z^{2}(y, \delta / 2)=z^{1}(y,-\delta / 2)=z^{s}(y) .
$$

As $\delta^{-1}\left(\partial_{x_{n}} z^{0}(y, \delta / 2)-\partial_{x_{n}} z^{0}(y,-\delta / 2)\right)=O(1)$ we cannot neglect this term in (A.4). Proceeding as above we thus obtain the model

$$
\partial_{t} z^{j}-\operatorname{div}_{g} c^{j} \nabla_{g} z^{j}=f^{j} \quad \text { in }(0, T) \times \Omega_{j}, j=1,2,
$$

and

$$
\left\{\begin{array}{l}
\partial_{t} z^{s}-\operatorname{div}^{s}\left(c^{s} \nabla^{s} z^{s}\right)=f^{s}+\frac{1}{\delta}\left(\left(c^{2} \partial_{x_{n}} z^{2}\right)_{\mid x_{n}=0^{+}}-\left(c^{1} \partial_{x_{n}} z^{1}\right)_{\mid x_{n}=0^{-}}\right), \\
z_{\mid x_{n}=0^{+}}^{2}=z^{s}=z_{\mid x_{n}=0^{-}}^{1},
\end{array}\right.
$$

in $(0, T) \times S$.

\section{Appendix B. Facts on semiclassical operators}

\section{B.1. Results for tangential semiclassical operators on $\mathbb{R}^{d}, d \geq 2$}

Semiclassical operators are defined in Section 1.4. Here, we provide the properties that we need in the main text. 
The composition formula for tangential symbols $b \in S_{\mathcal{T}}^{m}, b^{\prime} \in S_{\mathcal{T}}^{m^{\prime}}$ is given by

$$
\begin{gathered}
\left(b \# b^{\prime}\right)\left(z, \zeta^{\prime}\right)=(2 \pi h)^{-(d-1)} \iint e^{-i\left\langle t^{\prime}, \tau^{\prime}\right\rangle / h} b\left(z, \zeta^{\prime}+\tau^{\prime}, h\right) b^{\prime}\left(z^{\prime}+t^{\prime}, z_{d}, \zeta^{\prime}, h\right) d t^{\prime} d \tau^{\prime} \\
=\sum_{|\alpha|<N} \frac{(-i h)^{|\alpha|}}{\alpha !} \partial_{\zeta^{\prime}}^{\alpha} b\left(z, \zeta^{\prime}, h\right) \partial_{z^{\prime}}^{\alpha} b^{\prime}\left(z, \zeta^{\prime}, h\right)+r_{N}, \quad r_{N} \in h^{N} S_{\mathcal{T}}^{m+m^{\prime}-N},
\end{gathered}
$$

where

$$
\begin{aligned}
r_{N}=\frac{(-i h)^{N}}{(2 \pi h)^{(d-1)}} & \sum_{|\alpha|=N} \int_{0}^{1} \frac{N(1-s)^{N-1}}{\alpha !} \\
& \times \iint e^{-i\left\langle t^{\prime}, \tau^{\prime}\right\rangle / h} \partial_{\zeta^{\prime}}^{\alpha} b\left(z, \zeta^{\prime}+\tau^{\prime}, h\right) \partial_{z^{\prime}}^{\alpha} b^{\prime}\left(z^{\prime}+s t^{\prime}, z_{d}, \zeta^{\prime}, h\right) d t^{\prime} d \tau^{\prime} d s
\end{aligned}
$$

and yields a tangential symbol in $S_{\mathcal{T}}^{m+m^{\prime}}$.

If $s, m \in \mathbb{R}$ and $b \in S_{\mathcal{T}}^{m}$ we have the following regularity result:

$$
\left\|\Lambda_{\mathcal{T}}^{s} \mathrm{Op}_{\mathcal{T}}(b) u\right\|_{L^{2}\left(\mathbb{R}^{d}\right)} \leq C\left\|\Lambda_{\mathcal{T}}^{s+m} u\right\|_{L^{2}\left(\mathbb{R}^{d}\right)}, \quad u \in \mathscr{S}\left(\mathbb{R}^{d}\right) .
$$

We now consider the effect of change of variables.

Theorem B.1. Let $Z^{\prime}$ and $Z_{\kappa}^{\prime}$ be open subsets of $\mathbb{R}^{d-1}$ and let $\kappa: Z^{\prime} \rightarrow Z_{\kappa}^{\prime}$ be a diffeomorphism. If $b\left(z, \zeta^{\prime}, h\right) \in S_{\mathcal{T}}^{m}$ and the kernel of $\mathrm{Op}_{\mathcal{T}}(b)$ has support contained in $K \times \mathbb{R} \times K \times \mathbb{R}$, with $K$ compact and contained in $Z^{\prime}$, then the function

$$
b_{\kappa}\left(\hat{z}^{\prime}, z_{d}, \zeta^{\prime}, h\right)= \begin{cases}e^{-i\left\langle\kappa\left(z^{\prime}\right), \zeta^{\prime}\right\rangle / h} \mathrm{Op}_{\mathcal{T}}(b) e^{i\left\langle\kappa\left(z^{\prime}\right), \zeta^{\prime}\right\rangle / h} & \text { if } \hat{z}^{\prime}=\kappa\left(z^{\prime}\right) \in Z_{\kappa}^{\prime}, \\ 0 & \text { if } \hat{z}^{\prime} \notin Z_{\kappa}^{\prime}\end{cases}
$$

is in $S_{\mathcal{T}}^{m}$, and the kernel of $\mathrm{Op}_{\mathcal{T}}\left(b_{\kappa}\right)$ has support contained in $\kappa(K) \times \mathbb{R} \times \kappa(K) \times \mathbb{R}$, and

$$
(\kappa \otimes \mathrm{Id})^{*} \mathrm{Op}_{\mathcal{T}}\left(b_{\kappa}\right) u=\mathrm{Op}_{\mathcal{T}}(b)\left((\kappa \otimes \mathrm{Id})^{*} u\right), \quad u \in \mathscr{S}^{\prime}\left(\mathbb{R}^{d}\right) .
$$

For $b_{\kappa}$ we have the asymptotic expansion

$$
b_{\kappa}\left(\kappa\left(z^{\prime}\right), z_{d}, \zeta^{\prime}, h\right)-T_{\kappa, N}(b)\left(\kappa\left(z^{\prime}\right), z_{d}, \zeta^{\prime}, h\right) \in h^{N / 2} S_{\mathcal{T}}^{m-N / 2}
$$

with

$$
T_{\kappa, N}(b)\left(\kappa\left(z^{\prime}\right), z_{d}, \zeta^{\prime}, h\right)=\left.\sum_{\alpha<N} \frac{(-i h)^{|\alpha|}}{\alpha !} \partial_{\zeta^{\prime}}^{\alpha} b\left(z^{\prime}, z_{d},{ }^{t} \kappa\left(z^{\prime}\right)^{\prime} \zeta^{\prime}, h\right) \partial_{t^{\prime}}^{\alpha} e^{i\left\langle\rho_{z^{\prime}}\left(t^{\prime}\right), \zeta^{\prime}\right\rangle / h}\right|_{t^{\prime}=z^{\prime}}
$$

where $\rho_{z^{\prime}}\left(t^{\prime}\right)=\kappa\left(t^{\prime}\right)-\kappa\left(z^{\prime}\right)-\kappa^{\prime}\left(z^{\prime}\right)\left(t^{\prime}-z^{\prime}\right)$. 
A proof is provided in Appendix C.9. In particular we find that

$$
b_{\kappa}\left(\kappa\left(z^{\prime}\right), z_{d}, \zeta, h\right)=b\left(z^{\prime}, z_{d},{ }^{t} \kappa\left(z^{\prime}\right)^{\prime} \zeta^{\prime}, h\right)+h r\left(z^{\prime}, z_{d}, \zeta^{\prime}, h\right) \quad \text { with } r \in h S_{\mathcal{T}}^{m-1} \text {. }
$$

The principal symbol thus transforms as the regular pullback of a function defined in phase space (see Section 1.4.3).

Lemma B.2. Let $a \in S_{\mathcal{T}}^{m}$ be such that the kernel $K_{h}(z, t)=\mathcal{K}_{h, z_{d}}\left(z^{\prime}, t^{\prime}\right) \otimes \delta\left(z_{d}-t_{d}\right)$ of $\operatorname{Op}_{\mathcal{T}}(a)$ is such that $\mathcal{K}_{h, z_{d}}\left(z^{\prime}, t^{\prime}\right)$ vanishes if $\left|z^{\prime}-t^{\prime}\right| \leq \eta$ for some $\eta>0$. Then $a \in h^{\infty} S_{\mathcal{T}}^{-\infty}$.

Proof. We write, as an oscillatory integral,

$$
\mathcal{K}_{h, z_{d}}\left(z^{\prime}, t^{\prime}\right)=\frac{1}{(2 \pi h)^{d-1}} \int e^{i\left\langle z^{\prime}-t^{\prime}, \zeta^{\prime}\right\rangle / h} a\left(z, \zeta^{\prime}, h\right) d \zeta^{\prime} .
$$

Let $\chi \in \mathscr{C}^{\infty}\left(\mathbb{R}^{d-1}\right)$ be such that $\chi\left(z^{\prime}\right)=0$ if $\left|z^{\prime}\right| \leq \eta / 2$ and $\chi\left(z^{\prime}\right)=1$ if $\left|z^{\prime}\right| \geq \eta$. Then $\mathcal{K}_{h, z_{d}}\left(z^{\prime}, t^{\prime}\right)=\chi\left(z^{\prime}-t^{\prime}\right) \mathcal{K}_{h, z_{d}}\left(z^{\prime}, t^{\prime}\right)$. Hence, $\chi\left(z^{\prime}-t^{\prime}\right) a\left(z, \zeta^{\prime}, h\right)$ is an amplitude for $\operatorname{Op}_{\mathcal{T}}(a)$. The asymptotic series providing the associated symbol, which is in fact $a\left(z, \zeta^{\prime}, h\right)$, is (by [GS94])

$$
\left.a\left(z, \zeta^{\prime}, h\right) \sim \sum_{\alpha} \frac{(-i)^{|\alpha|} h^{\alpha}}{\alpha !} \partial_{t^{\prime}}^{\alpha} \partial_{\zeta^{\prime}}^{\alpha}\left(\chi\left(z^{\prime}-t^{\prime}\right) a\left(z, \zeta^{\prime}, h\right)\right)\right|_{t^{\prime}=z^{\prime}}
$$

Because of the support of $\chi$ the result follows.

\section{B.2. Semiclassical (tangential) operators on a manifold}

In the present article, we consider semiclassical operators that act on both the $x_{0}$ and $y$ variables, $x_{0} \in\left(0, X_{0}\right)$ and $y \in S$.

Let $\mathcal{X}$ be a manifold of the form $\left(0, X_{0}\right) \times S \times \mathbb{R}$. We denote by $\left(x_{0}, y, x_{n}\right)$ a typical element. We also set $\mathcal{X}^{\prime}=\left(0, X_{0}\right) \times S$. By abuse of notation we shall also denote by $\phi_{j}$ the map Id $\otimes \phi_{j} \otimes \mathrm{Id}\left(\right.$ resp. Id $\otimes \phi_{j}$ ) on $\mathbb{R} \times U_{j} \times \mathbb{R}\left(\right.$ resp. $\mathbb{R} \times U_{j}$ ); see Section 1.4.3 where the diffeomorphisms $\phi_{j}, j \in J$, are defined.

We recall the definition of a tangential semiclassical symbol in an open set $O \subset \mathbb{R}^{d}$.

Definition B.3. We say that $a\left(z, \zeta^{\prime}, h\right) \in S_{\mathcal{T}}^{m}\left(O \times \mathbb{R}^{d-1}\right)$ if, for any $\chi \in \mathscr{C}_{c}^{\infty}(O), \chi a \in$ $S_{\mathcal{T}}^{m}\left(\mathbb{R}^{d} \times \mathbb{R}^{d-1}\right)$.

We also recall the definition of tangential semiclassical symbols and operators on a manifold.

Definition B.4. 1. Let $m \in \mathbb{R}, j \in J$, and $a \in \mathscr{C}^{\infty}\left(T^{*}\left(\left(0, X_{0}\right) \times U_{j}\right) \times \mathbb{R}\right)$. We say that $a \in S_{\mathcal{T}}^{m}\left(T^{*}\left(\left(0, X_{0}\right) \times U_{j}\right) \times \mathbb{R}\right)$ if $\left(\phi_{j}^{-1}\right)^{*} a \in S_{\mathcal{T}}^{m}\left(\left(0, X_{0}\right) \times \tilde{U}_{j} \times \mathbb{R} \times \mathbb{R}^{n}\right)$.

2. Let $a \in \mathscr{C}^{\infty}\left(T^{*}\left(\mathcal{X}^{\prime}\right) \times \mathbb{R}\right)$. We say that $a \in S_{\mathcal{T}}^{m}\left(T^{*}\left(\mathcal{X}^{\prime}\right) \times \mathbb{R}\right)$ if, for all $j \in J$, $a_{\mid T^{*}\left(\left(0, X_{0}\right) \times U_{j}\right) \times \mathbb{R}} \in S_{\mathcal{T}}^{m}\left(T^{*}\left(\left(0, X_{0}\right) \times U_{j}\right) \times \mathbb{R}\right)$. 
Definition B.5. An operator $A: \mathscr{C}_{c}^{\infty}(\mathcal{X}) \rightarrow \mathscr{C}^{\infty}(\mathcal{X})$ is said to be tangential semiclassical on $\mathcal{X}$ of order $m \in \mathbb{R}$ if:

1. Its kernel is of the form

$$
K_{h}\left(x_{0}, y, x_{n} ; \hat{x}_{0}, \hat{y}, \hat{x}_{n}\right)=\mathcal{K}_{h, x_{n}}\left(x_{0}, y ; \hat{x}_{0}, \hat{y}\right) \otimes \delta\left(x_{n}-\hat{x}_{n}\right)
$$

2. Its kernel is regularizing outside $\operatorname{diag}(\mathcal{X} \times \mathcal{X})$ in the semiclassical sense: for all $\chi, \hat{\chi} \in$ $\mathscr{C}_{c}^{\infty}\left(\mathcal{X}^{\prime}\right)$ such that $\operatorname{supp}(\chi) \cap \operatorname{supp}(\hat{\chi})=\emptyset$ we have

$$
\chi\left(x_{0}, y\right) \hat{\chi}\left(\hat{x}_{0}, \hat{y}\right) \mathcal{K}_{h, x_{n}}\left(x_{0}, y ; \hat{x}_{0}, \hat{y}\right) \in \mathscr{C}^{\infty}\left(\mathcal{X}^{\prime} \times \mathcal{X}^{\prime}\right),
$$

and for all $N, \alpha \in \mathbb{N}$, and for any seminorm $q$ on $\mathscr{C}^{\infty}\left(\mathcal{X}^{\prime} \times \mathcal{X}^{\prime}\right)$ there exists $C=$ $C_{\chi, \hat{\chi}, N, \alpha, q}>0$ such that

$$
\sup _{x_{n} \in \mathbb{R}} q\left(\chi\left(x_{0}, y\right) \hat{\chi}\left(\hat{x}_{0}, \hat{y}\right) \partial_{x_{n}}^{\alpha} \mathcal{K}_{h, x_{n}}\left(x_{0}, y ; \hat{x}_{0}, \hat{y}\right)\right) \leq C h^{N}
$$

3. For all $j \in J$ and all $\lambda \in \mathscr{C}_{c}^{\infty}\left(\left(0, X_{0}\right) \times U_{j}\right), \tilde{\lambda} \in \mathscr{C}_{c}^{\infty}\left(\left(0, X_{0}\right) \times \tilde{U}_{j}\right)$, we have

$$
\mathscr{S}^{\prime}\left(\mathbb{R}^{n+1}\right) \ni u \mapsto\left(\phi_{j}^{-1}\right)^{*}(\lambda \otimes \mathrm{Id}) A \phi_{j}^{*}(\tilde{\lambda} \otimes \mathrm{Id}) u
$$

in $\Psi_{\mathcal{T}}^{m}\left(\mathbb{R}^{n+1}\right)$.

In this case, we write $A \in \Psi_{\mathcal{T}}^{m}(\mathcal{X})$.

Note that we shall often write $\lambda$ and $\tilde{\lambda}$ in place of $\lambda \otimes$ Id and $\tilde{\lambda} \otimes$ Id respectively.

We set

$$
\begin{aligned}
h^{\infty} S_{\mathcal{T}}^{-\infty}\left(\left(0, X_{0}\right) \times \tilde{U}_{j} \times \mathbb{R} \times \mathbb{R}^{n}\right) & =\bigcap_{N \in \mathbb{N}} h^{N} S_{\mathcal{T}}^{-N}\left(\left(0, X_{0}\right) \times \tilde{U}_{j} \times \mathbb{R} \times \mathbb{R}^{n}\right), \\
h^{\infty} \Psi_{\mathcal{T}}^{-\infty}\left(\left(0, X_{0}\right) \times \tilde{U}_{j} \times \mathbb{R}\right) & =\bigcap_{N \in \mathbb{N}} h^{N} \Psi_{\mathcal{T}}^{-N}\left(\left(0, X_{0}\right) \times \tilde{U}_{j} \times \mathbb{R}\right),
\end{aligned}
$$

Remark B.6. The first two points of Definition B.5 in fact state that the semiclassical wave front set of the kernel of the operator is confined to the conormal bundle of the diagonal of $\mathcal{X}$. As a consequence, $A$ maps $\mathscr{E}^{\prime}(\mathcal{X})$ into $\mathscr{D}^{\prime}(\mathcal{X})$ [Hör90, Theorem 8.2.13]. We also note that the same properties hold for the transpose (resp. adjoint) operator. If moreover $A$ is properly supported then $A$ maps

$\mathscr{C}_{c}^{\infty}(\mathcal{X}) \rightarrow \mathscr{C}_{c}^{\infty}(\mathcal{X}), \quad \mathscr{C}^{\infty}(\mathcal{X}) \rightarrow \mathscr{C}^{\infty}(\mathcal{X}), \quad \mathscr{E}^{\prime}(\mathcal{X}) \rightarrow \mathscr{E}^{\prime}(\mathcal{X}), \quad \mathscr{D}^{\prime}(\mathcal{X}) \rightarrow \mathscr{D}^{\prime}(\mathcal{X})$ continuously, and the same holds for ${ }^{t} A$.

Observe that tangential semiclassical differential operators naturally have all the properties listed above. 
Proposition B.7. If $A \in \Psi_{\mathcal{T}}^{m}(\mathcal{X})$, then for all $j \in J$, there exists $a_{j}\left(x_{0}, x^{\prime}, x_{n} ; \xi_{0}, \xi^{\prime}\right) \in$ $S_{\mathcal{T}}^{m}\left(\left(0, X_{0}\right) \times \tilde{U}_{j} \times \mathbb{R} \times \mathbb{R}^{n}\right)$ such that for all $\lambda \in \mathscr{C}_{c}^{\infty}\left(\left(0, X_{0}\right) \times U_{j}\right), \tilde{\lambda} \in \mathscr{C}_{c}^{\infty}\left(\left(0, X_{0}\right) \times \tilde{U}_{j}\right)$,

$$
\left(\phi_{j}^{-1}\right)^{*} \lambda A \phi_{j}^{*} \tilde{\lambda}-\mathrm{Op}_{\mathcal{T}}\left(\left(\left(\phi_{j}^{-1}\right)^{*} \lambda\right) a_{j}\right) \tilde{\lambda} \in h^{\infty} \Psi_{\mathcal{T}}^{-\infty}\left(\mathbb{R}^{n} \times \mathbb{R}\right) .
$$

Moreover, $a_{j}$ is uniquely defined up to $h^{\infty} S_{\mathcal{T}}^{-\infty}\left(\left(0, X_{0}\right) \times \tilde{U}_{j} \times \mathbb{R} \times \mathbb{R}^{n}\right)$.

We refer to Appendix C.10 for a proof. We say that $a_{j}$ is the (representative of the) local symbol of $A$ (modulo $h^{\infty} S_{\mathcal{T}}^{-\infty}$ ) in the chart $\left(0, X_{0}\right) \times \tilde{U}_{j} \times \mathbb{R}$. We find that the symbol of $\left(\phi_{j}^{-1}\right)^{*} \lambda A \phi_{j}^{*} \tilde{\lambda}$ is given by $\left(\left(\phi_{j}^{-1}\right)^{*} \lambda\right) a_{j} \# \tilde{\lambda}$ modulo $h^{\infty} S_{\mathcal{T}}^{-\infty}\left(\mathbb{R}^{n} \times \mathbb{R} \times \mathbb{R}^{n}\right)$, from the previous proposition. The symbols $\left(a_{j}\right)_{j \in J}$ follow the natural transformations when going from one chart to another.

Proposition B.8. If $U_{j} \cap U_{k} \neq \emptyset$, we introduce

$$
\tilde{U}_{j, k}=\phi_{j}\left(U_{j} \cap U_{k}\right) \subset \tilde{U}_{j} \quad \text { and } \quad \tilde{U}_{k, j}=\phi_{k}\left(U_{j} \cap U_{k}\right) \subset \tilde{U}_{k} .
$$

Let $A \in \Psi_{\mathcal{T}}^{m}(\mathcal{X})$ with $a_{j}$ as given in Proposition B.7. Then

$$
a_{k \mid\left(0, X_{0}\right) \times \tilde{U}_{k, j} \times \mathbb{R}}-T_{\phi_{j k}, N}\left(a_{j \mid\left(0, X_{0}\right) \times \tilde{U}_{j, k} \times \mathbb{R}}\right) \in h^{N} S_{\mathcal{T}}^{m-N / 2}\left(\left(0, X_{0}\right) \times \tilde{U}_{k, j} \times \mathbb{R} \times \mathbb{R}^{n}\right) .
$$

We refer to Appendix C.11 for a proof. The notation $T_{\phi_{j k}, N}$ is defined in (B.5). The open sets $\tilde{U}_{j, k}$ and $\tilde{U}_{k, j}$ are represented in Figure 2.

As a consequence, only considering the first term in the sum defining $T_{\phi_{j k}, N}\left(a_{j}\right)$, we observe that the principal part of $a_{j}$ defined on $\left(0, X_{0}\right) \times \tilde{U}_{j} \times \mathbb{R} \times \mathbb{R}^{n}$ transforms as a function on $T^{*}\left(\mathcal{X}^{\prime}\right) \times \mathbb{R}$ through a change of variables.

Let $A \in \Psi_{\mathcal{T}}^{m}(\mathcal{X})$ and let $a_{j}, j \in J$, be representatives of the local symbol (class) given in the local chart by Proposition B.7. We set $a=\sum_{j \in J} \psi_{j} \phi_{j}^{*} a_{j}$ and find

$$
a-\phi_{j}^{*} a_{j} \in h \Psi_{\mathcal{T}}^{m-1}\left(T^{*}\left(\left(0, X_{0}\right) \times U_{j}\right) \times \mathbb{R}\right) .
$$

This defines $a$ modulo $h S_{\mathcal{T}}^{m-1}\left(T^{*}\left(\mathcal{X}^{\prime}\right) \times \mathbb{R}\right)$.

Definition B.9. We define the principal symbol of $A$ as the class of $a$ in $S_{\mathcal{T}}^{m}\left(T^{*}\left(\mathcal{X}^{\prime}\right) \times\right.$ $\mathbb{R}) / h S_{\mathcal{T}}^{m-1}\left(T^{*}\left(\mathcal{X}^{\prime}\right) \times \mathbb{R}\right)$ and we denote it by $\sigma(A)$.

Proposition B.10. Let $A \in \Psi_{\mathcal{T}}^{m}(\mathcal{X}), B \in \Psi_{\mathcal{T}}^{m^{\prime}}(\mathcal{X})$ both be properly supported. Then $A B \in \Psi_{\mathcal{T}}^{m+m^{\prime}}(\mathcal{X})$ and (a representative of) its local symbol in any chart $\left(U_{j}, \phi_{j}\right)$ is given by $a_{j} \# b_{j}$ with the notation of Proposition B.7. In particular, $\sigma(A B)=\sigma(A) \sigma(B)$.

We refer to Appendix C.12 for a proof.

The following natural result is a consequence of what precedes.

Corollary B.11. If $A \in \Psi_{\mathcal{T}}^{m}(\mathcal{X})$ and $B \in \Psi_{\mathcal{T}}^{m^{\prime}}(\mathcal{X})$ are both properly supported then the commutator $[A, B] \in h \Psi_{\mathcal{T}}^{m+m^{\prime}-1}(\mathcal{X})$ and $(h / i)\{\sigma(A), \sigma(B)\}$ is (a representative of) its principal symbol. 
With the Sobolev norms defined in Section 1.4.3 we have the following result.

Proposition B.12. Let $A \in \Psi_{\mathcal{T}}^{\ell}(\mathcal{X})$ be properly supported, $\ell=0,1$. Let $K$ be a compact subset of $\mathcal{X}^{\prime}$. Then there exist $L$, a compact subset of $\mathcal{X}^{\prime}$, and $C>0$ such that for all $u \in \mathscr{C}_{c}^{\infty}\left(\mathcal{X}^{\prime}\right)$ with $\operatorname{supp}(u) \subset K$,

$$
\operatorname{supp}\left((A u)_{\mid x_{n}=0}\right) \subset L \quad \text { and } \quad\left|(A u)_{\mid x_{n}=0}\right|_{k} \leq C|u|_{\ell+k} \quad \text { with } k= \begin{cases}0 \text { or } 1 & \text { if } \ell=0 \\ 0 & \text { if } \ell=1 .\end{cases}
$$

We refer to Appendix C.13 for a proof. The norms in the proposition are those defined in (1.21).

\section{B.3. A particular class of semiclassical operators on $\mathcal{M}_{+}$}

In this section, we prove that the operators $\Xi_{\bullet}$ defined in $(3.24), \bullet=\mathscr{E}, \mathscr{F}, \mathscr{G}, \mathscr{Z}$, are tangential semiclassical pseudo-differential operators on $\mathcal{M}_{+}$. We also establish some properties of their symbols.

Let $\zeta^{0} \in \mathscr{C}_{c}^{\infty}\left(0, X_{0}\right)$ that satisfies $\zeta^{0}=1$ on a neighborhood of $\left(\alpha_{0}, X_{0}-\alpha_{0}\right)$ and $0 \leq \zeta^{0} \leq 1$. We set

$$
\zeta_{j}^{0}\left(x_{0}, y, x_{n}\right)=\zeta^{0}\left(x_{0}\right) \psi_{j}(y)
$$

For all $j \in J$, we choose $\tilde{\zeta}_{j}^{0} \in \mathscr{C}_{c}^{\infty}\left(\left(0, X_{0}\right) \times \tilde{U}_{j}\right)$ with $\tilde{\zeta}_{j}^{0}=1$ in a neighborhood of $\operatorname{supp}\left(\left(\phi_{j}^{-1}\right)^{*} \zeta_{j}^{0}\right)$.

Let $p \in S_{\mathcal{T}}^{m}\left(\mathcal{M}_{+}^{*}\right)$. We define, for some $j \in J$,

$$
p_{j}=\tilde{\zeta}_{j}^{0}\left(\phi_{j}^{-1}\right)^{*} p \quad \text { and } \quad Q=\phi_{j}^{*} \mathrm{Op}_{\mathcal{T}}\left(p_{j}\right)\left(\phi_{j}^{-1}\right)^{*} \zeta_{j}^{0}
$$

Lemma B.13. We have $Q \in \Psi_{\mathcal{T}}^{m}\left(\mathcal{M}_{+}\right)$. Moreover, denoting by $q_{k}$ (a representative of) the local symbol of $Q$ in the chart $\tilde{U}_{k}$, we have

1. $q_{j}=p_{j} \#\left(\left(\phi_{j}^{-1}\right)^{*} \zeta_{j}^{0}\right) \bmod h^{\infty} S_{\mathcal{T}}^{-\infty}\left(\mathbb{R}^{n} \times[0,2 \varepsilon] \times \mathbb{R}^{n}\right)$ and $q_{j}$ can be chosen such that $\operatorname{supp}\left(q_{j}\right) \subset \operatorname{supp}\left(\tilde{\zeta}_{j}^{0}\right) \times \mathbb{R}^{n} \times[0,2 \varepsilon] \subset \tilde{U}_{j} \times \mathbb{R}^{n} \times[0,2 \varepsilon]$;

2. $q_{k}=0$ if $U_{j} \cap U_{k}=\emptyset$;

3. $q_{k}=T_{\phi_{j k}, N}\left(q_{j}\right) \bmod h^{N / 2} S_{\mathcal{T}}^{m-N / 2}\left(\mathbb{R}^{n} \times[0,2 \varepsilon] \times \mathbb{R}^{n}\right)$ for all $N \in \mathbb{N}$, and $\operatorname{supp}\left(q_{k}\right) \subset$ $\phi_{k}\left(U_{j} \cap U_{k}\right) \times \mathbb{R}^{n} \times \mathbb{R}$ if $k \neq j$ and $U_{j} \cap U_{k} \neq \emptyset$.

Proof. Let us first check that $Q \in \Psi_{\mathcal{T}}^{m}\left(\mathcal{M}_{+}\right)$. The definition of $Q$ first yields $\operatorname{supp}\left(K_{Q, h}\right)$ $\subset\left(\left(0, X_{0}\right) \times U_{j} \times[0,2 \varepsilon]\right)^{2}$. Then, for $\lambda \in \mathscr{C}_{c}^{\infty}\left(\left(0, X_{0}\right) \times U_{j}\right), \tilde{\lambda} \in \mathscr{C}_{c}^{\infty}\left(\left(0, X_{0}\right) \times \tilde{U}_{j}\right)$, we have

$$
\left(\phi_{j}^{-1}\right)^{*} \lambda Q \phi_{j}^{*} \tilde{\lambda}=\left(\left(\phi_{j}^{-1}\right)^{*} \lambda\right) \mathrm{Op}_{\mathcal{T}}\left(p_{j}\right)\left(\left(\phi_{j}^{-1}\right)^{*} \zeta_{j}^{0}\right) \tilde{\lambda} \in \Psi_{\mathcal{T}}^{m}\left(\mathbb{R}^{n} \times[0,2 \varepsilon]\right),
$$

and the symbol of this operator is $\left(\left(\phi_{j}^{-1}\right)^{*} \lambda\right) \# p_{j} \#\left(\left(\phi_{j}^{-1}\right)^{*} \zeta_{j}^{0}\right) \tilde{\lambda}$. According to Proposition B.7, this yields $q_{j}=p_{j} \#\left(\phi_{j}^{-1}\right)^{*} \zeta_{j}^{0} \bmod h^{\infty} S_{\mathcal{T}}^{-\infty}\left(\mathbb{R}^{n} \times[0,2 \varepsilon] \times \mathbb{R}^{n}\right)$. The local 
representation $q_{j}$ can be chosen with compact support in $\tilde{U}_{j}$ since $p_{j}=\tilde{\zeta}_{j}^{0}\left(\phi_{j}^{-1}\right)^{*} p$ and $\operatorname{supp}\left(\tilde{\zeta}_{j}^{0}\right) \subset \tilde{U}_{j}$. As a consequence, the first point is satisfied.

Taking now $\lambda$ and $\tilde{\lambda}$ such that $\operatorname{supp}(\lambda) \cap \operatorname{supp}\left(\phi_{j}^{*} \tilde{\lambda}\right)=\emptyset$, we find $\left(\phi_{j}^{-1}\right)^{*} \lambda Q \phi_{j}^{*} \tilde{\lambda} \in$ $h^{\infty} \Psi_{\mathcal{T}}^{-\infty}\left(\mathbb{R}^{n} \times[0,2 \varepsilon]\right)$, so that the kernel of $Q$ satisfies (B.7). Next, we take $k \in J, k \neq j$ and $\lambda \in \mathscr{C}_{c}^{\infty}\left(\left(0, X_{0}\right) \times U_{k}\right), \tilde{\lambda} \in \mathscr{C}_{c}^{\infty}\left(\left(0, X_{0}\right) \times \tilde{U}_{k}\right)$ and compute $\left(\phi_{k}^{-1}\right)^{*} \lambda Q \phi_{k}^{*} \tilde{\lambda}$.

If $U_{j} \cap U_{k}=\emptyset$, this is the null operator and the second point is satisfied. If $U_{j} \cap U_{k} \neq \emptyset$, we take

- $\hat{\lambda}_{j} \in \mathscr{C}_{c}^{\infty}\left(\left(0, X_{0}\right) \times\left(U_{j} \cap U_{k}\right)\right)$ such that $\hat{\lambda}_{j}=1$ on $\operatorname{supp}\left(\phi_{j}^{*} \tilde{\zeta}_{j}^{0}\right) \cap \operatorname{supp}(\lambda)$,

- $\lambda_{j}^{(1)}, \lambda_{j}^{(2)} \in \mathscr{C}_{c}^{\infty}\left(\left(0, X_{0}\right) \times\left(U_{j} \cap U_{k}\right)\right)$ such that $\lambda_{j}^{(1)}=\lambda_{j}^{(2)}=1$ on $\operatorname{supp}\left(\zeta_{j}^{0}\right) \cap$ $\operatorname{supp}\left(\phi_{k}^{*} \tilde{\lambda}\right)$.

We have

$$
\left(\phi_{k}^{-1}\right)^{*} \lambda Q \phi_{k}^{*} \tilde{\lambda}=\left(\left(\phi_{k}^{-1}\right)^{*} \lambda\right)\left(\phi_{j k}^{-1}\right)^{*} \tilde{Q}\left(\phi_{j k}\right)^{*}\left(\left(\phi_{k}^{-1}\right)^{*} \lambda_{j}^{(1)}\right) \tilde{\lambda}
$$

where

$$
\tilde{Q}=\left(\left(\phi_{j}^{-1}\right)^{*} \hat{\lambda}_{j}\right) \mathrm{Op}_{\mathcal{T}}\left(p_{j}\right)\left(\left(\phi_{j}^{-1}\right)^{*} \zeta_{j}^{0} \lambda_{j}^{(2)}\right) .
$$

The kernel of the operator $\tilde{Q}$ has a compact support and we can hence apply Theorem B.1. According to the change of variables formula (B.4) the symbol of the operator $\left(\phi_{k}^{-1}\right)^{*} \lambda Q \phi_{k}^{*} \tilde{\lambda}$ is given by

$$
\begin{aligned}
\left(\phi_{k}^{-1}\right)^{*} \lambda \# T_{\phi_{j k}, N}\left(\left(\left(\phi_{j}^{-1}\right)^{*} \hat{\lambda}_{j}\right) \# p_{j} \#\left(\left(\phi_{j}^{-1}\right)^{*} \zeta_{j}^{0} \lambda_{j}^{(2)}\right)\right) \#\left(\left(\phi_{k}^{-1}\right)^{*} \lambda_{j}^{(1)}\right) \tilde{\lambda} \\
\\
\bmod h^{N / 2} S_{\mathcal{T}}^{m-N / 2}\left(\mathbb{R}^{n} \times[0,2 \varepsilon] \times \mathbb{R}^{n}\right) .
\end{aligned}
$$

Combining the definition of $T_{\phi_{j k}, N}$, the composition formula, and the definition of $q_{j}$ we find this symbol to be

$$
\begin{aligned}
\left(\phi_{k}^{-1}\right)^{*} \lambda \# T_{\phi_{j k}, N}\left(p_{j} \#\left(\phi_{j}^{-1}\right)^{*} \zeta_{j}^{0}\right) \# \tilde{\lambda} \bmod h^{N / 2} S_{\mathcal{T}}^{m-N / 2}\left(\mathbb{R}^{n} \times[0,2 \varepsilon] \times \mathbb{R}^{n}\right) \\
=\left(\phi_{k}^{-1}\right)^{*} \lambda \# T_{\phi_{j k}, N}\left(q_{j}\right) \# \tilde{\lambda} \bmod h^{N / 2} S_{\mathcal{T}}^{m-N / 2}\left(\mathbb{R}^{n} \times[0,2 \varepsilon] \times \mathbb{R}^{n}\right),
\end{aligned}
$$

because of the supports of $\hat{\lambda}_{j}, \lambda_{j}^{(1)}$ and $\lambda_{j}^{(2)}$. This proves the third point. Finally, we obtain $Q \in \Psi_{\mathcal{T}}^{m}\left(\mathcal{M}_{+}\right)$, which concludes the proof of the lemma.

Proposition B.14. Let $P=\sum_{j \in J} \phi_{j}^{*} \mathrm{Op}_{\mathcal{T}}\left(p_{j}\right)\left(\phi_{j}^{-1}\right)^{*} \zeta_{j}^{0}$ with $p_{j}=\tilde{\zeta}_{j}^{0}\left(\phi_{j}^{-1}\right)^{*} p$. Then $P \in \Psi_{\mathcal{T}}^{m}\left(\mathcal{M}_{+}\right)$and its principal symbol is $\sigma(P)\left(\mathrm{x}, \xi_{0}, \eta\right)=\zeta^{0}\left(x_{0}\right) p\left(\mathrm{x}, \xi_{0}, \eta\right)$. Moreover, in each chart $\tilde{U}_{k}$, there exists a (representative of the) local symbol of $P$ supported in $\operatorname{supp}\left(\zeta^{0} \phi_{k}^{*} p\right)$.

Proof. According to Lemma B.13, in the chart $\tilde{U}_{k}$, the local symbol of $P$ is

$$
p_{k} \#\left(\phi_{k}^{-1}\right)^{*} \zeta_{k}^{0}+\sum_{j \neq k} T_{\phi_{j k}, N}\left(p_{j} \#\left(\phi_{j}^{-1}\right)^{*} \zeta_{j}^{0}\right) \bmod h^{N / 2} S_{\mathcal{T}}^{m-N / 2}\left(\mathbb{R}^{n} \times[0,2 \varepsilon] \times \mathbb{R}^{n}\right)
$$


for all $N \in \mathbb{N}$. According to the composition formula (B.1) and the definition of $T_{\phi_{j k}, N}$ (B.5), the principal part of this local representation is

$$
\begin{aligned}
p_{k}\left(\phi_{k}^{-1}\right)^{*} \zeta_{k}^{0}+\sum_{j \neq k}\left(\phi_{j k}^{-1}\right)^{*}\left(p_{j}\left(\phi_{j}^{-1}\right)^{*} \zeta_{j}^{0}\right) & =\tilde{\zeta}_{k}^{0}\left(\phi_{k}^{-1}\right)^{*} p \zeta_{k}^{0}+\sum_{j \neq k}\left(\phi_{j k}^{-1}\right)^{*} \tilde{\zeta}_{j}^{0}\left(\phi_{j}^{-1}\right)^{*} p \zeta_{j}^{0} \\
& =\left(\left(\phi_{k}^{-1}\right)^{*} p\right) \sum_{j \in J}\left(\phi_{k}^{-1}\right)^{*} \zeta_{j}^{0}=\zeta^{0}\left(\phi_{k}^{-1}\right)^{*} p,
\end{aligned}
$$

since $\sum_{j \in J} \zeta_{j}^{0}=\zeta^{0}$, defined in Section 3.6. Moreover, for every $N \in \mathbb{N}$, the expression (B.9) is supported in the support of $\left(\phi_{k}^{-1}\right)^{*} p$. This property can be preserved by a representative of the asymptotic series as $N \rightarrow \infty$. This concludes the proof.

For the Sobolev norms introduced in Section 1.4.3 we have the following natural result.

Lemma B.15. Let $P$ be as in Proposition B.14, let $v \in \mathscr{C}^{\infty}\left(\mathcal{M}_{+}\right)$and set $u_{j}=$ $\mathrm{Op}_{\mathcal{T}}\left(p_{j}\right)\left(\phi_{j}^{-1}\right)^{*} \zeta_{j}^{0} v$. Then

$$
\|P v\|_{\ell} \lesssim \sum_{j \in J}\left\|u_{j}\right\|_{\ell}, \quad\left|(P v)_{\mid x_{n}=0^{+}}\right|_{\ell} \lesssim \sum_{j \in J}\left|u_{j \mid x_{n}=0^{+}}\right| \ell, \quad \ell=0,1 .
$$

Proof. We treat the case of norms in all dimensions. We have $P v=\sum_{j \in J} \phi_{j}^{*} u_{j}$. Then

$$
\|P v\|_{\ell} \leq \sum_{j \in J}\left\|\phi_{j}^{*} u_{j}\right\|_{\ell} .
$$

We then conclude with the use of Lemma 1.9.

\section{Appendix C. Proofs of some technical results}

\section{C.1. Proof of Lemma 1.8}

Let $\left(g_{j}\right)_{j}$ be a family of smooth functions on $S$ with $\operatorname{supp}\left(g_{j}\right) \subset U_{j}$ and $\sum_{j} g_{j}=g \geq$ $C>0$ in $S$. We set $M_{r}(u)=\sum_{j}\left|\left(\phi_{j}^{-1}\right)^{*} g_{j} u\right|_{\mathscr{H}^{r}\left(\mathbb{R}^{n-1}\right)}$. It is sufficient to prove that $\left|\left(\phi_{j}^{-1}\right)^{*} f_{j} u\right|_{\mathscr{H}}\left(\mathbb{R}^{n-1}\right) \leq C M_{r}(u)$ for some constant $C>0$.

We set $\tilde{g}_{j}=g_{j} / g$, which forms a partition of unity. We have

$$
\left|\left(\phi_{j}^{-1}\right)^{*} f_{j} u\right|_{\mathscr{C}^{r}\left(\mathbb{R}^{n-1}\right)} \leq \sum_{k}\left|\left(\phi_{j}^{-1}\right)^{*} f_{j} \tilde{g}_{k} u\right|_{\mathscr{C}^{r}\left(\mathbb{R}^{n-1}\right)} .
$$

Next

$$
\left|\left(\phi_{j}^{-1}\right)^{*} f_{j} \tilde{g}_{k} u\right|_{\mathscr{H} r\left(\mathbb{R}^{n-1}\right)} \leq C\left|\left(\phi_{k}^{-1}\right)^{*} f_{j} \tilde{g}_{k} u\right|_{\mathscr{H}^{r}\left(\mathbb{R}^{n-1}\right)}
$$

as $\phi_{j k}$ is a $\mathscr{C}^{\infty}$ diffeomorphism between $\phi_{j}\left(U_{j} \cap U_{k}\right)$ and $\phi_{k}\left(U_{j} \cap U_{k}\right)$. Introducing $\hat{g}_{k} \in \mathscr{C}_{c}^{\infty}\left(U_{k}\right)$ such that $\hat{g}_{k}=1$ on $\operatorname{supp}\left(g_{k}\right)$ we find

$$
\begin{aligned}
& \left|\left(\phi_{j}^{-1}\right)^{*} f_{j} \tilde{g}_{k} u\right|_{\mathscr{H}^{r}\left(\mathbb{R}^{n-1}\right)} \leq C\left|\left(\phi_{k}^{-1}\right)^{*} f_{j} \hat{g}_{k} g_{k} / g u\right|_{\mathscr{H}^{r}\left(\mathbb{R}^{n-1}\right)} \\
& =C\left|\left(\phi_{k}^{-1}\right)^{*}\left(f_{j} \hat{g}_{k} / g\right)\left(\phi_{k}^{-1}\right)^{*}\left(g_{k} u\right)\right|_{\mathscr{H}\left(\mathbb{R}^{n-1}\right)} \leq C^{\prime}\left|\left(\phi_{k}^{-1}\right)^{*}\left(g_{k} u\right)\right|_{\mathscr{H} r\left(\mathbb{R}^{n-1}\right)} \leq C^{\prime \prime} M_{r}(u),
\end{aligned}
$$

as $v \mapsto v\left(\phi_{k}^{-1}\right)^{*}\left(f_{j} \hat{g}_{k} / g\right)$ is continuous in $\mathscr{H}^{r}\left(\mathbb{R}^{n-1}\right)$. The proof is complete. 


\section{C.2. Proof of Proposition 2.2}

First we note that in the proof it suffices to consider the operator $A_{\delta}+\lambda$ Id for $\lambda$ sufficiently large, in place of $\mathcal{A}_{\delta}+\lambda$ Id. An inspection of the proof that follows also shows that piecewise $\mathscr{C}^{1}$ regularity of the coefficient $c$ and $\mathscr{C}^{1}$ regularity of $c^{s}$ are sufficient to prove the result.

We consider a finite open covering $\left(O_{j}\right)_{j}$ of $\bar{\Omega}$ together with a subordinated partition of unity $\sum_{j} \theta_{j}=1$ that satisfies moreover, if $O_{j} \cap S \neq \emptyset$ :

1. we can choose local coordinates in $O_{j}$ such that $S$ is given by $\left\{x_{n}=0\right\}$.

2. $\partial_{\eta} \theta_{j \mid S}=0$, i.e. $\theta_{j}$ is flat at $S$ in the normal direction to $S$.

The result of Proposition 2.2 is clear away from $S$ by standard elliptic regularity theory. We thus consider $O=O_{j}$ such that $O_{j} \cap S \neq \emptyset$. With $\theta=\theta_{j}$ we set $v=\theta z$ and $v^{s}=\theta z^{s}$ and $V=\left(v, v^{s}\right)$. From (2.3) we have

$$
\|V\|_{\mathcal{H}_{\delta}^{1}} \lesssim\|F\|_{\mathcal{H}_{\delta}^{0}}
$$

The result will be achieved if we prove

$$
\sum_{i=1,2}\left|v_{\mid \Omega_{i}}\right|_{H^{2}\left(O \cap \Omega_{i}\right)}+\delta^{1 / 2}\left|v^{s}\right|_{H^{2}(O \cap S)} \lesssim\|F\|_{\mathcal{H}_{\delta}^{0}}
$$

uniformly in $\delta$.

We write $c \nabla_{g} v=c\left(\nabla_{g} \theta\right) z+c \theta\left(\nabla_{g} z\right)$. If $\psi \in H_{0}^{1}(O)$ we have

$\left(c \nabla_{g} v, \nabla_{g} \psi\right)_{L^{2}(O)}=\left(c\left(\nabla_{g} \theta\right) z, \nabla_{g} \psi\right)_{L^{2}(O)}+\left(c\left(\nabla_{g} z\right) \theta, \nabla_{g} \psi\right)_{L^{2}(O)}$

$=-\sum_{i=1,2}\left(\nabla_{g}\left(c\left(\nabla_{g} \theta\right) z\right), \psi\right)_{L^{2}\left(O \cap \Omega_{i}\right)}+\left(c \nabla_{g} z, \nabla_{g}(\theta \psi)\right)_{L^{2}(O)}-\left(c\left(\nabla_{g} z \nabla_{g} \theta\right), \psi\right)_{L^{2}(O)}$,

with an integration by parts using that $\partial_{\eta} \theta_{\mid S}=0$.

Similarly for $\psi^{s} \in H_{0}^{1}(O \cap S)$ we have

$$
\begin{aligned}
\left(c^{s} \nabla^{s} v^{s}, \nabla^{s} \psi^{s}\right)_{L^{2}(O \cap S)}= & -\left(\nabla^{s}\left(c^{s}\left(\nabla^{s} \theta\right) z^{s}\right), \psi^{s}\right)_{L^{2}(O \cap S)}+\left(c^{s} \nabla^{s} z^{s}, \nabla^{s}\left(\theta \psi^{s}\right)\right)_{L^{2}(O \cap S)} \\
& -\left(c^{s}\left(\nabla^{s} z^{s} \nabla^{s} \theta\right), \psi^{s}\right)_{L^{2}(O \cap S)}
\end{aligned}
$$

Considering the weak problem (2.2) satisfied by $Z$ we thus obtain

$$
\begin{gathered}
\left(c \nabla_{g} v, \nabla_{g} \psi\right)_{L^{2}(O)}+\delta\left(c^{s} \nabla^{s} v^{s}, \nabla^{s} \psi^{s}\right)_{L^{2}(O \cap S)}+\lambda(v, \psi)_{L^{2}(O)}+\delta \lambda\left(v^{s}, \psi^{s}\right)_{L^{2}(O \cap S)} \\
=(\phi, \psi)_{L^{2}(O)}+\delta\left(\phi^{s}, \psi^{s}\right)_{L^{2}(O \cap S)}
\end{gathered}
$$

for $\Psi=\left(\psi, \psi^{s}\right) \in \mathcal{H}_{\delta}^{1}$, where $\Phi=\left(\phi, \phi^{s}\right) \in L^{2} \times L^{2}$ with

$$
\begin{aligned}
\phi_{\mid \Omega_{i}} & =\left(-\nabla_{g}\left(c\left(\nabla_{g} \theta\right) z\right)-c\left(\nabla_{g} z \nabla_{g} \theta\right)+\theta f\right)_{\mid \Omega_{i}}, \quad i=1,2, \\
\phi^{s} & =-\nabla^{s}\left(c^{s}\left(\nabla^{s} \theta\right) z^{s}\right)-c^{s}\left(\nabla^{s} z^{s} \nabla^{s} \theta\right)+\theta f^{s},
\end{aligned}
$$

and by (2.3) we have

$$
\|\Phi\|_{\mathcal{H}_{\delta}^{0}} \lesssim\|F\|_{\mathcal{H}_{\delta}^{0}}
$$


We now make a local change of variable in $O$ such that $S$ becomes $\left\{x_{n}=0\right\}$. The weak problem that $\left(v, v^{s}\right)$ satisfies takes the form

$$
\begin{aligned}
& \sum_{k, l} \int_{O} \underline{c}_{k, l} \partial_{x_{k}} v \partial_{x_{l}} \bar{\psi} d x+\delta \sum_{k, l}^{\prime} \int_{O \cap S} \underline{c}_{k, l}^{s} \partial_{x_{k}} v^{s} \partial_{x_{l}} \bar{\psi}^{s} d x+\lambda \int_{O} \alpha v \bar{\psi} d x+\lambda \delta \int_{O \cap S} \beta v^{s} \bar{\psi}^{s} d x \\
&=\int_{O} \alpha \phi \bar{\psi} d x+\delta \int_{O \cap S} \beta \phi^{s} \bar{\psi}^{s} d x, \quad \Psi=\left(\psi, \psi^{s}\right) \in \mathcal{H}_{\delta}^{1}, \quad(\mathrm{C} .4)
\end{aligned}
$$

where $\sum_{k, l}$ is a sum with $k, l$ running over $\{1, \ldots, n\}$ and $\sum_{k, l}^{\prime}$ is a sum with $k, l$ running over $\{1, \ldots, n-1\}$. The functions $\alpha$ and $\beta$ come from Jacobians. The functions $\underline{c}_{k, l}$ are piecewise $\mathscr{C}^{1}$ with a discontinuity across the interface $S$, and the functions $\underline{c}_{k, l}^{s}$ are $\mathscr{C}^{1}$. Note that $v \in H^{1}(O)$ and $v^{s} \in H^{1}(O \cap S)$ with supports finitely away from $\partial O$.

We now use the Nirenberg translation method. Let $h$ be parallel to $S$. Define $D_{h}$ by $D_{h}(\rho)=(\rho(x+h)-\rho(x)) /|h|$. Observe that $D_{-h}\left(D_{h} v\right) \in H_{0}^{1}(O)$ and $D_{-h}\left(D_{h} v^{s}\right) \in$ $H_{0}^{1}(O \cap S)$ for $|h|$ sufficiently small and set $\psi=D_{-h}\left(D_{h} v\right)$ and $\psi^{s}=D_{-h}\left(D_{h} v^{s}\right)$. As $\left(D_{h} f_{1} f_{2}\right)=f_{1}(x+h) D_{h} f_{2}+\left(D_{h} f_{1}\right) f_{2}$ this yields

$$
\begin{aligned}
\sum_{k, l} \int_{O} \underline{c}_{k, l}(x+ & h) \partial_{x_{k}} D_{h} v \partial_{x_{l}} D_{h} \bar{v} d x+\delta \sum_{k, l}^{\prime} \int_{O \cap S} \underline{c}_{k, l}^{s}(x+h) \partial_{x_{k}} D_{h} v^{s} \partial_{x_{l}} D_{h} \bar{v}^{s} d x \\
& +\lambda \int_{O} \alpha(x+h)\left|D_{h} v\right|^{2} d x+\lambda \delta \int_{O \cap S} \beta(x+h)\left|D_{h} v^{s}\right|^{2} d x \\
& +\sum_{k, l} \int_{O}\left(D_{h} \underline{c}_{k, l}\right) \partial_{x_{k}} v \partial_{x_{l}} D_{h} \bar{v} d x+\delta \sum_{k, l}^{\prime} \int_{O \cap S}\left(D_{h} \underline{c}_{k, l}^{s}\right) \partial_{x_{k}} v^{s} \partial_{x_{l}} D_{h} \bar{v}^{s} d x \\
& +\lambda \int_{O}\left(D_{h} \alpha\right) v D_{h} \bar{v} d x+\delta \lambda \int_{O \cap S}\left(D_{h} \beta\right) v^{s} D_{h} \bar{v}^{s} d x \\
& =\int_{O} \alpha \phi D_{-h}\left(D_{h} \bar{v}\right) d x+\delta \int_{O \cap S} \beta \phi^{s} D_{-h}\left(D_{h} \bar{v}^{s}\right) d x
\end{aligned}
$$

We note that

$$
\begin{aligned}
& \mid \sum_{k, l} \int_{O}\left(D_{h} \underline{c}_{k, l}\right) \partial_{x_{k}} v \partial_{x_{l}} D_{h} \bar{v} d x+\delta \sum_{k, l}^{\prime} \int_{O \cap S}\left(D_{h} \underline{c}_{k, l}^{s}\right) \partial_{x_{k}} v^{s} \partial_{x_{l}} D_{h} \bar{v}^{s} d x \\
& \quad+\lambda \int_{O}\left(D_{h} \alpha\right) v D_{h} \bar{v} d x+\delta \lambda \int_{O \cap S}\left(D_{h} \beta\right) v^{s} D_{h} \bar{v}^{s} d x \mid \lesssim\|V\|_{\mathcal{H}_{\delta}^{1}}\left\|D_{h} V\right\|_{\mathcal{H}_{\delta}^{1}}
\end{aligned}
$$

If $\rho \in H_{0}^{1}(O)$ with support finitely away from the boundary $\partial O$ then $\left|D_{h}(\rho)\right|_{L^{2}(O)}$ $\leq|\nabla \rho|_{L^{2}(O)}$ for $|h|$ sufficiently small [Bre83, Proposition IX.3]. We thus have

$$
\left|\int_{O} \alpha \phi D_{-h}\left(D_{h} \bar{v}\right) d x+\delta \int_{O \cap S} \beta \phi^{s} D_{-h}\left(D_{h} \bar{v}^{s}\right) d x\right| \lesssim\|\Phi\|_{\mathcal{H}_{\delta}^{0}}\left\|D_{h} V\right\|_{\mathcal{H}_{\delta}^{1}} .
$$


We thus find

$$
\left|a_{\lambda}\left(D_{h} v, D_{h} v\right)\right| \lesssim\left(\|V\|_{\mathcal{H}_{\delta}^{1}}+\|\Phi\|_{\mathcal{H}_{\delta}^{0}}\right)\left\|D_{h} V\right\|_{\mathcal{H}_{\delta}^{1}} \lesssim\|\Phi\|_{\mathcal{H}_{\delta}^{0}}\left\|D_{h} V\right\|_{\mathcal{H}_{\delta}^{1}}
$$

uniformly in $\delta$, using (C.1). The coercivity of $a_{\lambda}$ gives

$$
\left\|D_{h} V\right\|_{\mathcal{H}_{\delta}^{1}} \lesssim\|F\|_{\mathcal{H}_{\delta}^{0}}
$$

For $k \in\{1, \ldots, n\}$ and $\psi \in \mathscr{C}_{c}^{\infty}(O)$, we choose $h$ in the direction of the $x_{l}$ coordinate, $l \in\{1, \ldots, n-1\}$. Then

$$
\left|\left(\partial_{x_{k}} v, D_{-h} \psi\right)_{L^{2}(O)}\right|=\left|\left(D_{h} \partial_{x_{k}} v, \psi\right)_{L^{2}(O)}\right| \leq\left\|D_{h} V\right\|_{\mathcal{H}_{\delta}^{1}}\|\psi\|_{L^{2}} \lesssim\|F\|_{\mathcal{H}_{\delta}^{0}}\|\psi\|_{L^{2}}
$$

As the 1.h.s. converges to $\left|\left(\partial_{x_{k}} v, \partial_{x_{l}} \psi\right)_{L^{2}(O)}\right|$ we see that $\partial_{x_{k} x_{l}}^{2} v \in L^{2}$ and

$$
\left\|\partial_{x_{k} x_{l}}^{2} v\right\|_{L^{2}} \lesssim\|F\|_{\mathcal{H}_{\delta}^{0}}, \quad(k, l) \neq(n, n) .
$$

Similarly for $k \in\{1, \ldots, n-1\}$ and $\psi^{s} \in \mathscr{C}_{c}^{\infty}(O \cap S)$, we choose $h$ in the direction of the $x_{l}$ coordinate, $l \in\{1, \ldots, n-1\}$. We have

$$
\begin{aligned}
\delta^{1 / 2}\left|\left(\partial_{x_{k}} v^{s}, D_{-h} \psi^{s}\right)_{L^{2}(O \cap S)}\right| & =\delta^{1 / 2}\left|\left(D_{h} \partial_{x_{k}} v^{s}, \psi^{s}\right)_{L^{2}(O \cap S)}\right| \leq\left\|D_{h} V\right\|_{\mathcal{H}_{\delta}^{1}}\left\|\psi^{s}\right\|_{L^{2}} \\
& \lesssim\|F\|_{\mathcal{H}_{\delta}^{0}}\left\|\psi^{s}\right\|_{L^{2}},
\end{aligned}
$$

and we obtain $\partial_{x_{k} x_{l}}^{2} v^{s} \in L^{2}(O)$ and moreover

$$
\delta^{1 / 2}\left\|\partial_{x_{k} x_{l}}^{2} v^{s}\right\|_{L^{2}(O)} \lesssim\|F\|_{\mathcal{H}_{\delta}^{0}}, \quad k, l \in\{1, \ldots, n-1\} .
$$

From (C.4) observe now that (in a weak sense) we have in $\Omega_{i} \cap O, i=1,2$,

$$
\partial_{x_{n}}^{2} v=-\frac{1}{\underline{c}_{n n}}\left(\sum_{(k, l) \neq(n, n)} \partial_{x_{k}} \underline{c}_{k l} \partial_{x_{l}} v+\alpha \phi+\left(\partial_{x_{n}} \underline{c}_{n n}\right) \partial_{x_{n}} v-\lambda \alpha v\right) .
$$

It follows that $\partial_{x_{n}}^{2} v_{\mid \Omega_{i}} \in L^{2}\left(\Omega_{i}\right)$ and

$$
\left\|\partial_{x_{n}}^{2} v_{\mid \Omega_{i}}\right\|_{L^{2}\left(\Omega_{i}\right)} \lesssim\|F\|_{\mathcal{H}_{\delta}^{0}}, \quad i=1,2,
$$

which concludes the proof.

\section{C.3. Proof of Proposition 2.3}

An inspection of the proof shows that is sufficient to assume that $c$ is piecewise $\mathscr{C}^{m+1}$, $c^{s}$ is $\mathscr{C}^{m+1}$ and $S$ is of class $\mathscr{C}^{m+2}$. We proceed by induction. The case $m=0$ is treated in Proposition 2.2. Let $m_{0}>0$. Assume the result is true for $0 \leq m \leq m_{0}-1$ and 
$f_{\mid \Omega_{i}} \in H^{m_{0}}\left(\Omega_{i}\right), i=0,1$, and $f^{s} \in H^{m_{0}}(S)$. We thus have $Z=\left(z, z^{s}\right) \in H^{m_{0}+1}\left(\Omega_{1} \cup\right.$ $\left.\Omega_{2}\right) \times H^{m_{0}+1}(S)$ with

$$
\begin{aligned}
\sum_{i=1,2}\left\|z_{\mid} \Omega_{i}\right\|_{H^{m_{0}+1}\left(\Omega_{i}\right)}+\delta^{1 / 2}\left|z^{s}\right|_{H^{m_{0}+1}(S)} & \\
& \lesssim \sum_{i=1,2}\left\|f_{\mid \Omega_{i}}\right\|_{H^{m_{0}}\left(\Omega_{i}\right)}+\delta^{1 / 2}\left|f^{s}\right|_{H^{m_{0}}(S)}=N_{m_{0}}(F) .
\end{aligned}
$$

We use the same partition of unity $\theta_{j}, j=1, \ldots, N$, as in the proof of Proposition 2.2. Since the result is known away from $S$ by standard elliptic regularity theory, we consider $O=O_{j}$ such that $O_{j} \cap S \neq \emptyset$. With $\theta=\theta_{j}$ we set $v=\theta z$ and $v^{s}=\theta z^{s}$ and $V=$ $\left(v, v^{s}\right)$. With the notation of the proof of Proposition 2.2 we obtain, after a local change of variables,

$$
\begin{aligned}
& \sum_{k, l} \int_{O} \underline{c}_{k, l} \partial_{x_{k}} v \partial_{x_{l}} \bar{\psi} d x+\delta \sum_{k, l}^{\prime} \int_{O \cap S} \underline{c}_{k, l}^{s} \partial_{x_{k}} v^{s} \partial_{x_{l}} \bar{\psi}^{s} d x+\lambda \int_{O} \alpha v \bar{\psi} d x \\
&+\delta \lambda \int_{O \cap S} \beta v^{s} \bar{\psi}^{s} d x=\int_{O} \alpha \phi \bar{\psi} d x+\delta \int_{O \cap S} \beta \phi^{s} \bar{\psi}^{s} d x
\end{aligned}
$$

for $\Psi=\left(\psi, \psi^{s}\right) \in \mathcal{H}_{\delta}^{1}$, where $\sum_{k, l}$ is a sum with $k, l$ running over $\{1, \ldots, n\}$ and $\sum_{k, l}^{\prime}$ is a sum with $k, l$ running over $\{1, \ldots, n-1\}$. We have $v \in H^{m_{0}+1}(O)$ and $v^{s} \in$ $H^{m_{0}+1}(O \cap S)$ with supports finitely away from $\partial O$, and $\Phi=\left(\phi, \phi^{s}\right)$ is such that

$$
\sum_{i=1,2}\left\|\phi_{\mid \Omega_{i}}\right\|_{H^{m_{0}\left(\Omega_{i}\right)}}+\delta^{1 / 2}\left|\phi^{s}\right|_{H^{m_{0}}(S)} \lesssim N_{m_{0}}(F) .
$$

The functions $\underline{c}_{k, l}$ are piecewise $\mathscr{C}^{m+1}$ with a discontinuity across the interface $S$, and the functions $\underline{c}_{k, l}^{s}$ are $\mathscr{C}^{m+1}$.

For $j=1, \ldots, n-1$, if $\Psi$ is chosen such that

$$
\psi=\partial_{x_{j}} \tilde{\psi}, \quad \psi^{s}=\partial_{x_{j}} \tilde{\psi}^{s}, \quad \text { with }\left(\tilde{\psi}, \tilde{\psi}^{s}\right) \in\left(\mathscr{C}_{c}^{\infty}(O) \times \mathscr{C}_{c}^{\infty}(S \cap O)\right) \cap \mathcal{H}_{\delta}^{1},
$$

since $\partial_{x_{j}} v_{\mid \Omega_{i}} \in H^{1}\left(\Omega_{i}\right)$ and $\partial_{x_{j}} v^{s} \in H^{1}(S)$, we find

$$
\begin{aligned}
\sum_{k, l} \int_{O} \underline{c}_{k, l} \partial_{x_{k} x_{j}}^{2} v \partial_{x_{l}} \overline{\tilde{\psi}} d x+\delta & \sum_{k, l}^{\prime} \int_{O \cap S} \underline{c}_{k, l}^{s} \partial_{x_{k} x_{j}}^{2} v^{s} \partial_{x_{l}} \overline{\tilde{\psi}^{s}} d x+\lambda \int_{O} \alpha \partial_{x_{j}} v \bar{\psi} d x \\
& +\delta \lambda \int_{O \cap S} \beta \partial_{x_{j}} v^{s} \bar{\psi}^{s} d x=\int_{O} \tilde{\phi} \overline{\tilde{\psi}} d x+\delta \int_{O \cap S} \tilde{\phi}^{s} \overline{\tilde{\psi}^{s}} d x
\end{aligned}
$$

with

$$
\sum_{i=1,2}\left\|\tilde{\phi}_{\mid \Omega_{i}}\right\|_{H^{m_{0}-1}\left(\Omega_{i}\right)}+\delta^{1 / 2}\left|\tilde{\phi}^{s}\right|_{H^{m_{0}-1}(S)} \lesssim N_{m_{0}}(F)
$$


The induction assumption then yields ${ }^{6}$

$$
\sum_{i=1,2}\left\|\partial_{x_{j}} v_{\mid \Omega_{i}}\right\|_{H^{m_{0}+1}\left(\Omega_{i} \cap O\right)}+\delta^{1 / 2}\left|\partial_{x_{j}} v^{s}\right|_{H^{m_{0}+1}(S \cap O)} \lesssim N_{m_{0}}(F) .
$$

From (C.5) we have in $\Omega_{i} \cap O, i=1,2$, in a weak sense,

$$
\partial_{x_{n}}^{2} v=-\frac{1}{\underline{c}_{n n}}\left(\sum_{(k, l) \neq(n, n)} \partial_{x_{k}} \underline{c}_{k l} \partial_{x_{l}} v+\alpha \phi+\left(\partial_{x_{n}} \underline{c}_{n n}\right) \partial_{x_{n}} v-\lambda \alpha v\right) .
$$

As $v_{\mid \Omega_{i}} \in H^{2}\left(\Omega_{i}\right)$, this also holds in $L^{2}\left(\Omega_{i}\right)$. Thus $\partial_{x_{n}}^{2} v_{\mid \Omega_{i}} \in H^{m_{0}}\left(\Omega_{i} \cap O\right)$ and

$$
\left\|\partial_{x_{n}}^{2} v_{\mid \Omega_{i}}\right\|_{H^{m_{0}\left(\Omega_{i}\right)}} \lesssim N_{m_{0}}(F)
$$

by (C.6). This concludes the proof.

\section{C.4. Proof of Lemma 3.6}

The proof we give extends that of Lemma 3, p. 480 in [LR97]. We drop the " $r / l$ " notation here since the same argument holds for both cases. We have $p_{\varphi}=\xi_{n}^{2}+2 i\left(\partial_{x_{n}} \varphi\right) \xi_{n}+$ $q_{2}+2 i q_{1}$. We pick $\alpha \in \mathbb{C}$ such that $\alpha^{2}=\left(\partial_{x_{n}} \varphi\right)^{2}+q_{2}+2 i q_{1}$. Then the imaginary parts of the two roots of $p_{\varphi}$ are $-\partial_{x_{n}} \varphi \pm \operatorname{Re}(\alpha)$ and have opposite signs if and only if $|\operatorname{Re}(\alpha)|>\left|\partial_{x_{n}} \varphi\right|$. We note that

$$
|\operatorname{Re}(z)|>A \Leftrightarrow \operatorname{Re}\left(z^{2}\right)>A^{2}-\frac{\left(\operatorname{Im}\left(z^{2}\right)\right)^{2}}{4 A^{2}}, \quad z \in \mathbb{C},
$$

with a similar equivalence in the case of equalities on both sides. Substituting $\alpha$ for $z$, and $\left|\partial_{x_{n}} \varphi\right|$ for $A$, we thus find that the imaginary parts of the roots have opposite signs if and only if $\mu>0$, as $\mu=q_{2}+q_{1}^{2} /\left(\partial_{x_{n}} \varphi\right)^{2}$. In the case $\mu=0$ only one of the roots is real and the imaginary part of the second one has the opposite sign to $\partial_{x_{n}} \varphi$. In the case $\mu<0$ both imaginary parts of the roots have the same sign, opposite to the sign of $\partial_{x_{n}} \varphi$.

If $\operatorname{Im}\left(\rho^{+}\right) \geq C_{0}>0$ and $\operatorname{Im}\left(\rho^{-}\right) \leq-C_{0}$ then $|\operatorname{Re}(\alpha)| \geq\left|\partial_{x_{n}} \varphi\right|+C_{0}$ and by (C.7) we obtain

$$
\left(\partial_{x_{n}} \varphi\right)^{2}+q_{2}=\operatorname{Re}\left(\alpha^{2}\right) \geq\left(\left|\partial_{x_{n}} \varphi\right|+C_{0}\right)^{2}-\frac{q_{1}^{2}}{\left(\left|\partial_{x_{n}} \varphi\right|+C_{0}\right)^{2}},
$$

which gives

$$
\mu \geq C_{0}^{2}+2 C_{0}\left|\partial_{x_{n}} \varphi\right|+q_{1}^{2}\left(\frac{1}{\left(\partial_{x_{n}} \varphi\right)^{2}}-\frac{1}{\left(\left|\partial_{x_{n}} \varphi\right|+C_{0}\right)^{2}}\right) \geq C>0 .
$$

Conversely, assume that $\mu \geq C_{1}>0$. Note that for all $M>0$, there exists $R>0$ such that $\left|\xi_{0}\right|+|\eta|_{g} \geq R \Rightarrow|\operatorname{Re}(\alpha)| \geq M$. Actually, we have

$$
\operatorname{Re}\left(\alpha^{2}\right)-M^{2}+\frac{\operatorname{Im}\left(\alpha^{2}\right)^{2}}{4 M^{2}}>0
$$

\footnotetext{
6 The induction assumption is applied to the local form of the elliptic problem here, i.e., (C.5).
} 
for $\left|\xi_{0}\right|+|\eta|_{g} \geq R$ sufficiently large, which yields $|\operatorname{Re}(\alpha)| \geq M$ by (C.7). Taking now $M=\left|\partial_{x_{n}} \varphi\right|+C$, we obtain $\left|\operatorname{Im}\left(\rho^{ \pm}\right)\right| \geq C$.

It suffices to take $\left|\xi_{0}\right|+|\eta|_{g} \leq R, x_{0} \in\left[0, X_{0}\right], x_{n} \in[-2 \varepsilon, 2 \varepsilon]$. The variables $\left(x_{0}, y, x_{n}, \xi_{0}, \eta\right)$ such that $\mu \geq C_{1}$ are in a compact set $K$. Thus, $\min _{K}\left|\operatorname{Im}\left(\rho^{ \pm}\right)\right|$is reached. Finally, $\mu \geq C_{1}$ implies $\left|\operatorname{Im}\left(\rho^{ \pm}\right)\right| \geq C>0$ as $\operatorname{Im}\left(\rho^{ \pm}\right)$does not vanish if $\mu>0$. This concludes the proof of the first part of the lemma.

We now address the last point of the lemma. Let $0<l<L<\inf _{\overline{V_{\varepsilon}^{+}}}\left|\partial_{x_{n}} \varphi\right|$ and let $H=L^{2}-l^{2}$. In the region $\{\mu \geq-H\}$ we have

$\mu \geq l^{2}-\left(\partial_{x_{n}} \varphi\right)^{2} \geq\left(l^{2}-\left(\partial_{x_{n}} \varphi\right)^{2}\right)\left(1+\frac{q_{1}^{2}}{l^{2}\left(\partial_{x_{n}} \varphi\right)^{2}}\right)=l^{2}-\left(\partial_{x_{n}} \varphi\right)^{2}+q_{1}^{2}\left(\frac{1}{\left(\partial_{x_{n}} \varphi\right)^{2}}-\frac{1}{l^{2}}\right)$.

Since $\mu=q_{2}+q_{1}^{2} /\left(\partial_{x_{n}} \varphi\right)^{2}$ we then have $q_{2}+\left(\partial_{x_{n}} \varphi\right)^{2} \geq l^{2}-q_{1}^{2} / l^{2}$, which by (C.7) yields $|\operatorname{Re}(\alpha)| \geq l$. We conclude by observing that $\left|\rho^{+}-\rho^{-}\right| \geq\left|\operatorname{Im} \rho^{+}-\operatorname{Im} \rho^{-}\right|=2|\operatorname{Re}(\alpha)|$.

\section{C.5. Proof of Lemma 3.8}

We follow the notation of the proof of Lemma 3.6 above and again we drop the " $r / h$ " notation since the same argument holds for both cases. We choose $\alpha \in \mathbb{C}$ such that $\alpha^{2}=\left(\partial_{x_{n}} \varphi\right)^{2}+q_{2}+2 i q_{1}=r\left(x, \xi_{0}, \eta\right)-r\left(x, \partial_{x_{0}} \varphi, d_{y} \varphi\right)+2 i \tilde{r}\left(x, \xi_{0}, \eta, \partial_{x_{0}} \varphi, d_{y} \varphi\right)$, which implies the roots are $-i \partial_{x_{n}} \varphi \pm i \alpha$. We set $\lambda_{\mathcal{T}}=\left(1+\xi_{0}^{2}+|\eta|_{g}^{2}\right)^{1 / 2} \in S_{\mathcal{T}}^{1}\left(\mathcal{M}_{+}^{*}\right)$ and write $(\alpha / \lambda \mathcal{T})^{2}=v_{1}+v_{2}$ with

$$
\nu_{1}=\frac{r\left(x, \xi_{0}, \eta\right)}{\lambda_{\mathcal{T}}^{2}} \quad \text { and } \nu_{2}=\frac{1}{\lambda_{\mathcal{T}}^{2}}\left(-r\left(x, \partial_{x_{0}} \varphi, d_{y} \varphi\right)+2 i \tilde{r}\left(x, \xi_{0}, \eta, \partial_{x_{0}} \varphi, d_{y} \varphi\right)\right) .
$$

To prove the first result, i.e., $\chi \rho^{ \pm} \in S_{\mathcal{T}}^{1}\left(\mathcal{M}_{+}^{*}\right)$, it suffices to consider $\lambda_{\mathcal{T}}$ large, as we already know that the two roots are smooth in $\operatorname{supp}(\chi)$. Note that there exists $L>0$ such that $\left|\nu_{1}\right| \geq 2 L$, and $\left|\nu_{2}\right| \leq L$ for $\lambda_{\mathcal{T}}$ large, say $\lambda_{\mathcal{T}} \geq R_{1}$. In this region we have $\operatorname{Re}\left(\alpha^{2} / \lambda_{\mathcal{T}}^{2}\right) \geq v_{1}-\left|\operatorname{Re}\left(v_{2}\right)\right| \geq L$. In particular,

$$
\operatorname{Re}(\alpha / \lambda \mathcal{T}) \geq C>0 .
$$

If $\lambda_{\mathcal{T}} \geq R_{1}$, we have thus shown that $\left(\alpha / \lambda_{\mathcal{T}}\right)^{2}$ remains away from a neighborhood of the

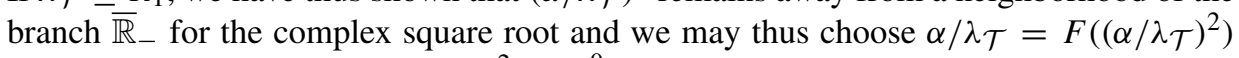
with $F=\mathscr{C}^{\infty}(\mathbb{C})$. Since $\left(\alpha / \lambda_{\mathcal{T}}\right)^{2} \in S_{\mathcal{T}}^{0}\left(\mathcal{M}_{+}^{*}\right)$, it follows from Theorem 18.1.10 in [Hör85a] that $\alpha / \lambda_{\mathcal{T}} \in S_{\mathcal{T}}^{0}\left(\mathcal{M}_{+}^{*}\right)$ for $\lambda_{\mathcal{T}} \geq R_{1}$, which yields the first conclusion.

Let $C_{0}>0$ and consider the region $\left\{\mu \geq C_{0}\right\}$. By Lemma 3.6 we have $\operatorname{Im}\left(\rho^{+}\right) \geq C$ $>0$ and $\operatorname{Im}\left(\rho^{-}\right) \leq-C$. By (C.8), we obtain $\left|\operatorname{Im}\left(\rho^{ \pm}\right)\right| \geq C \lambda \mathcal{T}$. Since $\operatorname{Im}\left(\rho^{+}\right)-\operatorname{Im}\left(\rho^{-}\right)$ $=2 \operatorname{Re}(\alpha)$, and since $\left|\operatorname{Im}\left(\rho^{+}\right)-\operatorname{Im}\left(\rho^{-}\right)\right| \geq C$, we obtain the final result from (C.8).

\section{C.6. Proof of Lemma 4.2}

Using (3.24) we have

$$
\mathrm{Op}_{\mathcal{T}}\left(\chi_{\mathscr{G}, j}\right)\left(\phi_{j}^{-1}\right)^{*} \zeta_{j} \tilde{\Xi}_{\mathscr{G} \mathscr{F}} v^{r}=\left(\phi_{j}^{-1}\right)^{*} \Xi_{\mathscr{G}, j} \tilde{\Xi}_{\mathscr{G} \mathscr{F}} v^{r} .
$$


We then write

$$
\operatorname{Op}_{\mathcal{T}}\left(\chi_{\mathscr{G}, j}\right)\left(\phi_{j}^{-1}\right)^{*} \zeta_{j} \tilde{\Xi}_{\mathscr{G} \mathscr{F}} v^{r}=\left(\phi_{j}^{-1}\right)^{*} \Xi_{\mathscr{G}, j} v^{r}-\left(\phi_{j}^{-1}\right)^{*} \Xi_{\mathscr{G}, j}\left(1-\tilde{\Xi}_{\mathscr{G} \mathscr{F}}\right) v^{r} .
$$

Note that $u_{\mathscr{G}, j}=\left(\phi_{j}^{-1}\right)^{*} \Xi_{\mathscr{G}, j} v^{r}$ by (4.1). We have $\Xi_{\mathscr{G}, j}\left(1-\tilde{\Xi}_{\mathscr{G} \mathscr{F}}\right) \in h^{\infty} \Psi_{\mathcal{T}}^{-\infty}\left(\mathcal{M}_{+}\right)$, as their local symbols in every chart have disjoint supports by Proposition B.14, because of the supports of $\zeta^{3}$ and $\tilde{\chi} \mathscr{G} \mathscr{F}$. This concludes the proof.

\section{C.7. Proof of Lemma 4.5}

Here, all functions are evaluated at the interface, i.e. $x_{n}=0^{+}$. From (4.67) we have

$$
\tilde{\sigma}_{\delta}=\tilde{\sigma}_{\delta}^{(0)}+\tilde{r}
$$

with

$$
\tilde{\sigma}_{\delta}^{(0)}=2 \partial_{x_{n}} \varphi_{j}^{r}\left|\sigma_{\delta}\right|^{2}+4 q_{1, j}^{r} \operatorname{Re}\left(\sigma_{\delta}\right)-2\left(\partial_{x_{n}} \varphi_{j}^{r}\right) q_{2, j}^{r}
$$

and $\tilde{r} \in\left(\delta^{2} / h\right) S_{\mathcal{T}}^{3}+\delta S_{\mathcal{T}}^{2}+h S_{\mathcal{T}}^{1}$, according to the definitions of $\tilde{\Sigma}_{\delta}$ in (4.69), $\sigma_{\delta}$ in (4.67), and $B_{1}^{r}, B_{1}^{r \prime}, B_{2}^{r}$ in (4.59). Observe that $\hat{\chi} \frac{h^{2}}{\delta^{2}+h^{2}} \tilde{\sigma}_{\delta}^{(0)} \in S_{\mathcal{T}}^{0}$ and the remainder satisfies

$$
\hat{\chi} \frac{h^{2}}{\delta^{2}+h^{2}} \tilde{r} \in h S_{\mathcal{T}}^{0},
$$

since $\left\langle\left(\xi_{0}, \xi^{\prime}\right)\right\rangle \leq C$ in $\operatorname{supp}(\hat{\chi})$.

Now, let us produce a lower bound for the symbol $\tilde{\sigma}_{\delta}^{(0)}$. Recalling the definition of $\mu^{r}$ in (3.12), denoting $\mu_{j}^{r}=\left(\phi_{j}^{-1}\right)^{*} \mu^{r}$ and $\vartheta=-i \frac{\delta c_{j}^{s}}{h c_{j}^{l}} p_{\varphi, j}^{s}$, we find

$$
\begin{aligned}
\tilde{\sigma}_{\delta}^{(0)} & =\frac{2}{\beta^{2}}\left(\partial_{x_{n}} \varphi_{j}^{r}\right)\left|\vartheta-\rho_{j}^{l,+}+k\right|^{2}+\frac{4}{\beta} \operatorname{Re}\left(\vartheta-\rho_{j}^{l,+}+k\right) q_{1, j}^{r}-2\left(\partial_{x_{n}} \varphi_{j}^{r}\right) q_{2, j}^{r} \\
& =2 \partial_{x_{n}} \varphi_{j}^{r}\left(\left|\left(\vartheta-\rho_{j}^{l,+}+k\right) / \beta+q_{1, j}^{r} / \partial_{x_{n}} \varphi_{j}^{r}\right|^{2}-\mu_{j}^{r}\right) \\
& \geq 2 \partial_{x_{n}} \varphi_{j}^{r}\left(\left(\operatorname{Im}\left(-\vartheta+\rho_{j}^{l,+}-k\right) / \beta\right)^{2}-\mu_{j}^{r}\right),
\end{aligned}
$$

since $\partial_{x_{n}} \varphi_{j}^{r} \geq C>0$ and $q_{1, j}^{r}$ is real. Hence

$$
\operatorname{Im}\left(-\vartheta+\rho_{j}^{l,+}-k\right)=\frac{\delta c_{j}^{s}}{h c_{j}^{l}} \operatorname{Re} p_{\varphi}^{s}+\left(\operatorname{Im} \rho_{j}^{l,+}-\operatorname{Im} \rho_{j}^{l,-}\right) / 2+\beta \partial_{x_{n}} \varphi_{j}^{r},
$$

as (4.10) gives $-\operatorname{Im} k=\partial_{x_{n}} \varphi_{j}^{l}+\beta \partial_{x_{n}} \varphi_{j}^{r}$ and the properties of the roots of the polynomial $p_{\varphi}^{r / l}$ given in (3.10) yield $\partial_{x_{n}} \varphi_{j}^{l}=-\left(\operatorname{Im} \rho_{j}^{l,+}+\operatorname{Im} \rho_{j}^{l,-}\right) / 2$. The first point of Lemma 3.6 gives $\operatorname{Im} \rho_{j}^{l,+}-\operatorname{Im} \rho_{j}^{l,-} \geq 0$, and thus

$$
\operatorname{Im}\left(-\vartheta+\rho_{j}^{l,+}-k\right) \geq K_{2} \delta / h+\beta \partial_{x_{n}} \varphi_{j}^{r},
$$


since in the present region, $\operatorname{Re}\left(p_{\varphi, j}^{s}\right)$ is positive elliptic by Proposition 3.5 and (3.11). Using condition (4.51), i.e., $\left(\partial_{x_{n}} \varphi_{j}^{r}\right)^{2}-\mu_{j}^{r} \geq K_{1}>0$ we find

$$
\tilde{\sigma}_{\delta}^{(0)} \geq 2 \partial_{x_{n}} \varphi_{j}^{r}\left(\frac{\delta^{2} K_{2}^{2}}{h^{2} \beta^{2}}+K_{1}\right) .
$$

This together with (C.9) concludes the proof.

\section{C.8. Proof of Lemma 4.7}

Let $X=\left(x_{0}, x^{\prime}, \xi_{0}, \xi^{\prime}\right) \in W$ and $\tilde{X}=\left(\tilde{x}_{0}, \tilde{x}^{\prime}, \tilde{\xi}_{0}, \tilde{\xi}^{\prime}\right) \in W$. If

$$
g_{W, X}(X-\tilde{X})=\left|\left(x_{0}, x^{\prime}\right)-\left(\tilde{x}_{0}, \tilde{x}^{\prime}\right)\right|^{2}+\frac{\left|\left(\xi_{0}, \xi^{\prime}\right)-\left(\tilde{\xi}_{0}, \tilde{\xi}^{\prime}\right)\right|^{2}}{\left\langle\left(\xi_{0}, \xi^{\prime}\right)\right\rangle^{2}}<r^{2},
$$

then, for $r$ sufficiently small, we have $C^{-1} \leq\left\langle\left(\tilde{\xi}_{0}, \tilde{\xi}^{\prime}\right)\right\rangle /\left\langle\left(\xi_{0}, \xi^{\prime}\right)\right\rangle \leq C$ for some $C>0$. As a consequence, $C^{-1} \leq \Lambda(\tilde{X}) / \Lambda(X) \leq C$ with $h, \delta>0$ arbitrary. Hence $\Lambda$ is slowly varying.

Next,

$$
\left\langle\left(\tilde{\xi}_{0}, \tilde{\xi}^{\prime}\right)\right\rangle^{2} \lesssim\left\langle\left(\xi_{0}, \xi^{\prime}\right)\right\rangle^{2}\left(1+\left|\left(\xi_{0}, \xi^{\prime}\right)-\left(\tilde{\xi}_{0}, \tilde{\xi}^{\prime}\right)\right|^{2}\right)
$$

so that

$$
\Lambda(\tilde{X})^{2} / \Lambda(X)^{2} \lesssim\left(1+\left|\left(\xi_{0}, \xi^{\prime}\right)-\left(\tilde{\xi}_{0}, \tilde{\xi}^{\prime}\right)\right|^{2}\right) \lesssim 1+g_{W, X}^{\sigma}(X-\tilde{X})
$$

for $h, \delta>0$ arbitrary. Here $g_{W}^{\sigma}$ denotes the dual metric on $W, g_{W}^{\sigma}=\left\langle\left(\xi_{0}, \xi^{\prime}\right)\right\rangle^{2}\left|d\left(x_{0}, x^{\prime}\right)\right|^{2}$ $+\left|d\left(\xi_{0}, \xi^{\prime}\right)\right|^{2}$. Hence, the order function $\Lambda$ is temperate, which concludes the proof.

\section{C.9. Proof of Theorem B.1: change of variables for semiclassical operators}

Here we consider operators on the whole space $\mathbb{R}^{n}$ of the form

$$
a\left(x, D_{x}, \tau\right)=u(x)=\iint e^{i\langle x-y, \xi\rangle} a(x, \xi, \tau) u(y) d y d \xi, \quad d \xi=(2 \pi)^{-n} d \xi
$$

where $a(x, \xi, \tau)$ is smooth in $x$ and $\xi$ and satisfies, for some $m \in \mathbb{R}$,

$$
\left|\partial_{x}^{\alpha} \partial_{\xi}^{\beta} a(x, \xi, \tau)\right| \leq C_{\alpha, \beta} \mu^{m-|\beta|}, \quad \mu^{2}=\tau^{2}+|\xi|^{2}, \tau \geq 1 .
$$

We say that $a \in S\left(\mu^{m}\right)$. We shall prove a change of variables formula for this kind of operator. We choose this form of operator to make use of parts of existing proofs. Operators of the form (C.10) are also called semiclassical. 
We recall that the semiclassical operators we consider in the main part of the article, i.e., with a small parameter $h$, can be put in the form (C.10). In fact, with $a(x, \xi, h) \in S^{m}$, we write

$$
\begin{aligned}
\mathrm{Op}(a) u(x) & =(2 \pi h)^{-n} \iint e^{i\langle x-y, \xi\rangle / h} a(x, \xi, h) u(y) d y d \xi \\
& =\iint e^{i\langle x-y, \xi\rangle} a(x, h \xi, h) u(y) d y d \xi
\end{aligned}
$$

and we have $\left|\partial_{x}^{\alpha} \partial_{\xi}^{\beta} a(x, h \xi, h)\right| \lesssim h^{|\beta|}\langle h \xi\rangle^{m-|\beta|}$. With $\tau=1 / h$ we find

$$
\left|\partial_{x}^{\alpha} \partial_{\xi}^{\beta} \tau^{m} a(x, h \xi, h)\right| \lesssim \tau^{m-|\beta|}(1+|\xi| / \tau)^{m-|\beta|} \lesssim \mu^{m-|\beta|}
$$

Hence, the symbol $h^{-m} a(x, h \xi, h)$ satisfies (C.11).

Theorem B. 1 is the translation for semiclassical tangential operators with a small parameter $h$ of the following theorem.

Theorem C.1. Let $X$ and $X_{\kappa}$ be open subsets of $\mathbb{R}^{n}$ and let $\kappa: X \rightarrow X_{\kappa}$ be a diffeomorphism. If $a \in S\left(\mu^{m}\right)$ and the kernel of $a\left(x, D_{x}, \tau\right)$ has compact support in $X \times X$ then the function

$$
a_{\kappa}(y, \eta, \tau)= \begin{cases}e^{-i\langle\kappa(x), \eta\rangle} a\left(x, D_{x}, \tau\right) e^{i\langle\kappa(x), \eta\rangle} & \text { if } y=\kappa(x) \in X_{\kappa}, \\ 0 & \text { if } y \notin X_{\kappa},\end{cases}
$$

is in $S\left(\mu^{m}\right)$, the kernel of $a_{\kappa}\left(x, D_{x}, \tau\right)$ has compact support in $X_{\kappa} \times X_{\kappa}$, and

$$
\left(a_{\kappa}\left(x, D_{x}, \tau\right) u\right) \circ \kappa=a\left(x, D_{x}, \tau\right)(u \circ \kappa), \quad u \in \mathscr{S}^{\prime}\left(\mathbb{R}^{n}\right) .
$$

For $a_{\kappa}$ we have the asymptotic expansion

$a_{\kappa}(\kappa(x), \eta, \tau)-\sum_{\alpha<N} \frac{(-i)^{|\alpha|}}{\alpha !} \partial_{\xi}^{\alpha} a\left(x,{ }^{t} \kappa(x)^{\prime} \eta, \tau\right) \partial_{y}^{\alpha} e^{i\left\langle\rho_{x}(y), \eta\right\rangle}{ }_{\mid y=x} \in S\left(\mu^{m-N / 2}\right)$,

where $\rho_{x}(y)=\kappa(y)-\kappa(x)-\kappa^{\prime}(x)(y-x)$.

Note that $\rho_{x}(y)$ vanishes to second order at $x$ and the terms in the series are in $S\left(\mu^{m-|\alpha| / 2}\right)$. In fact the order of each term in the asymptotic series (C.14) is explained by the following result that we shall use below.

Lemma C.2. We can write $\partial_{y}^{\alpha} e^{i\left\langle\rho_{x}(y), \eta\right\rangle}$ as a linear combination of terms

$$
\prod_{j \in \mathcal{I}}\left\langle x-y, \rho_{x}, j(y) \eta\right\rangle \prod_{j \in \mathcal{J}}\left\langle\partial_{y}^{\alpha_{j}} \rho_{x}(y), \eta\right\rangle e^{i\left\langle\rho_{x}(y), \eta\right\rangle},
$$

for some matrix-valued function $\rho_{x, j}, j \in \mathcal{I} \cup \mathcal{J}$, with $\left|\alpha_{j}\right| \geq 2$ if $j \in \mathcal{J}, k=|\mathcal{I}|$ and $\ell=$ $|\mathcal{I}|+|\mathcal{J}|$ such that $k \leq \ell \leq|\alpha|$ and $\ell \leq(|\alpha|+k) / 2$. In particular, $\left|\partial_{y}^{\alpha} e^{i\left\langle\rho_{x}(y), \eta\right\rangle}\right|_{x=y} \mid \leq$ $C_{\alpha}\langle\eta\rangle^{\alpha / 2}$. 
Proof. We note that $\partial_{y}^{\alpha}\left(e^{i\left\langle\rho_{x}(y), \eta\right\rangle}\right)$ can be written as a linear combination of terms of the form

$$
e^{i\left\langle\rho_{x}(y), \eta\right\rangle} \prod_{1 \leq j \leq p}\left\langle\partial_{y}^{\alpha_{j}} \rho_{x}(y), \eta\right\rangle \quad \text { with } \sum_{1 \leq j \leq p}\left|\alpha_{j}\right|=|\alpha|, \quad p \leq|\alpha|, \quad\left|\alpha_{j}\right| \geq 1
$$

We set $\mathcal{I}=\left\{1 \leq j \leq p ;\left|\alpha_{j}\right|=1\right\}$ and $\mathcal{J}=\left\{1 \leq j \leq p ;\left|\alpha_{j}\right| \geq 2\right\}$. We have $|\mathcal{I}|+|\mathcal{J}|=p \leq|\alpha|$ and moreover $|\alpha| \geq|\mathcal{I}|+2|\mathcal{J}|$, which gives $|\mathcal{I}|+|\mathcal{J}| \leq(|\alpha|+|\mathcal{I}|) / 2$. As $\rho_{x}(y)$ vanishes to second order at $y=x$ we obtain $\left\langle\partial_{y}^{\alpha_{j}} \rho_{x}(y), \eta\right\rangle=\left\langle x-y, \rho_{x, j}(y) \eta\right\rangle$ for some function $\rho_{x, j}$ if $j \in \mathcal{I}$.

Proof of Theorem C.1. Let the kernel of $a\left(x, D_{x}, \tau\right)$ be supported in $K \times K, K \subset X$, compact. In particular $a(x, \xi, \tau)=0$ if $x \notin K$. Let $\phi \in \mathscr{C}_{c}^{\infty}(X)$ be such that $\phi=1$ in a neighborhood of $K$, and $\tilde{\phi} \in \mathscr{C}_{c}^{\infty}(X)$ be such that $\tilde{\phi}=1$ in a neighborhood of $\operatorname{supp}(\phi)$. Here, we follow the proof of Theorem 18.1.17 in [Hör85a], and we first find that for $\tau$ fixed formula (C.13) holds for $a_{\kappa}$ given by (C.12). Moreover $a_{\kappa}$ is smooth with respect to $x$ and $\xi$ and we have

$a_{\kappa}(\kappa(x), \xi)=\phi(x) \iint e^{i\langle x-y, \xi\rangle+i\langle\kappa(y)-\kappa(x), \eta\rangle} a(x, \xi, \tau) \tilde{\phi}(y) d y d \xi, \quad x \in X$

It thus remains to prove that $a_{\kappa} \in S\left(\mu^{m}\right)$ and the asymptotic representation (C.14) holds.

For the proof we shall distinguish two regimes: $\tau \lesssim|\eta|$ and $\tau \gtrsim|\eta|$. We thus introduce $w \in \mathscr{C}_{c}^{\infty}(\mathbb{R})$ such that $w=1$ in a neighborhood of 0 and set

$$
\gamma_{1}(x, \eta, \tau)=w(\tau /\langle\eta\rangle) a_{\kappa}(\kappa(x), \eta, \tau), \quad \gamma_{2}(x, \eta, \tau)=(1-w)(\tau /\langle\eta\rangle) a_{\kappa}(\kappa(x), \eta, \tau) .
$$

We shall prove the two propositions below.

Proposition C.3. We have $\gamma_{1}(x, \eta, \tau) \in S\left(\mu^{m}\right)$ and

$$
\gamma_{1}(x, \eta, \tau)-w(\tau /\langle\eta\rangle) \sum_{\alpha<N} \frac{(-i)^{|\alpha|}}{\alpha !} \partial_{\xi}^{\alpha} a\left(x,{ }^{t} \kappa(x)^{\prime} \eta\right) \partial_{y}^{\alpha} e^{i\left\langle\rho_{x}(y), \eta\right\rangle} \mid y=x \in S\left(\mu^{m-N / 2}\right) .
$$

Proposition C.4. We have $\gamma_{2}(x, \eta, \tau) \in S\left(\mu^{m}\right)$ and

$\gamma_{2}(x, \eta, \tau)-(1-w)(\tau /\langle\eta\rangle) \sum_{\alpha<N} \frac{(-i)^{|\alpha|}}{\alpha !} \partial_{\xi}^{\alpha} a\left(x,{ }^{t} \kappa(x)^{\prime} \eta\right) \partial_{y}^{\alpha} e^{i\left\langle\rho_{x}(y), \eta\right\rangle} \mid y=x \in S\left(\mu^{m-N / 2}\right)$.

With these two results, Theorem C.1 clearly follows as $\kappa$ is a diffeomorphism.

We shall need the following result in the course of the proofs, which is the counterpart of Proposition 18.1.4 in [Hör85a] for semiclassical symbols. 
Lemma C.5. Let $a_{j}(x, \xi, \tau) \in S\left(\mu^{m_{j}}\right), j \in \mathbb{N}$, with $m_{j} \rightarrow-\infty$ as $j \rightarrow \infty$. Let $a(x, \xi, \tau)$ be smooth with respect to $x$ and $\xi$ such that for all $\alpha, \beta$, for some $C>0$ and $v$ depending on $\alpha$ and $\beta$,

$$
\left|\partial_{x}^{\alpha} \partial_{\xi}^{\beta} a(x, \xi, \tau)\right| \leq C \mu^{\nu}, \quad x, \xi \in \mathbb{R}^{n}, \tau \geq 1 .
$$

Assume there is a sequence $v_{k} \rightarrow-\infty$ such that

$$
\left|a(x, \xi, \tau)-\sum_{j<k} a_{j}(x, \xi, \tau)\right| \leq C_{k} \mu^{\nu_{k}}, \quad x, \xi \in \mathbb{R}^{n}, \tau \geq 1 .
$$

Then $a \in S\left(\mu^{m}\right), m=\sup m_{j}$, and $a(x, \xi, \tau)-\sum_{j<k} a_{j}(x, \xi, \tau) \in S\left(\mu^{m_{k}^{\prime}}\right)$ with $m_{k}^{\prime}=$ $\max _{j \geq k} m_{j}$.

The proof of Lemma C.5 is similar to that of Proposition 18.1.4 in [Hör85a]. It is left to the reader.

Proof of Proposition C.3. We have

$$
\begin{aligned}
& \gamma_{1}(x, \eta, \tau)=w(\tau /\langle\eta\rangle) a_{\kappa}(\kappa(x), \xi) \\
& \quad=w(\tau /\langle\eta\rangle) \phi(x) \iint e^{i\langle x-y, \xi\rangle+i\langle\kappa(y)-\kappa(x), \eta\rangle} a(x, \xi, \tau) \tilde{\phi}(y) d y d \xi, \quad x \in X .
\end{aligned}
$$

Let $\max \left(\left|\kappa^{\prime}(y)\right|,\left|\kappa^{\prime}(y)^{-1}\right|\right) \leq C_{0}$. Setting $\Phi(\xi, \eta)=\int e^{i\langle\kappa(y), \eta\rangle-i\langle y, \xi\rangle} \tilde{\phi}(y) d y$, one obtains, by a nonstationary phase argument [Hör85a, p. 82],

$$
|\Phi(\xi, \eta)| \leq C_{N}(1+|\xi|+|\eta|)^{-N} \quad \text { if }|\xi| \leq \frac{|\eta|}{2 C_{0}} \text { or }|\xi| \geq 2 C_{0}|\eta|
$$

Let then $\chi(\xi) \in \mathscr{C}_{c}^{\infty}\left(\mathbb{R}^{n}\right)$ be equal to 1 if $\left(2 C_{0}\right)^{-1}<|\xi|<2 C_{0}$, and equal to 0 if $|\xi|<\left(4 C_{0}\right)^{-1}$, and set $\gamma_{1}=I_{1}+I_{2}$ with

$$
\begin{aligned}
& I_{1}(x, \eta, \tau) \\
& \quad=w(\tau /\langle\eta\rangle) \phi(x) \iint e^{i\langle x-y, \xi\rangle+i\langle\kappa(y)-\kappa(x), \eta\rangle} a(x, \xi, \tau) \tilde{\phi}(y)(1-\chi)(\xi /|\eta|) d y d \xi \\
& \quad=w(\tau /\langle\eta\rangle) \phi(x) e^{-i\langle\kappa(x), \eta\rangle} \int e^{i\langle x, \xi\rangle} a(x, \xi, \tau) \Phi(\xi, \eta)(1-\chi)(\xi /|\eta|) d \xi
\end{aligned}
$$

and

$$
\begin{aligned}
I_{2}= & w(\tau /\langle\eta\rangle) \phi(x) \iint e^{i\langle x-y, \xi\rangle+i\langle\kappa(y)-\kappa(x), \eta\rangle} a(x, \xi, \tau) \tilde{\phi}(y) \chi(\xi /|\eta|) d y d \xi \\
= & w(\tau /\langle\eta\rangle) \phi(x) \omega^{n} e^{-i\langle\kappa(x), \eta\rangle} \\
& \times \iint e^{i \omega\langle x-y, \xi\rangle+i \omega\langle\kappa(y), \eta / \omega\rangle} a(x, \omega \xi, \tau) \tilde{\phi}(y) \chi(\xi) d y d \xi, \quad \omega=|\eta| .
\end{aligned}
$$


From (C.21) and as $\tau \lesssim|\eta|$ here we find

$$
\left|I_{1}(x, \eta, \tau)\right| \leq C_{N}|w(\tau /\langle\eta\rangle)| \int(\tau+|\xi|)^{m}(1+|\xi|+|\eta|)^{-(N+n+1+m)} d \xi, \quad N \in \mathbb{N}
$$

which gives

$$
\left|I_{1}(x, \eta, \tau)\right| \leq \begin{cases}C_{N}|w(\tau /\langle\eta\rangle)| \int(|\eta|+|\xi|)^{m}(1+|\xi|+|\eta|)^{-(N+n+1+m)} d \xi & \text { if } m \geq 0, \\ C_{N}|w(\tau /\langle\eta\rangle)| \int(1+|\xi|)^{m}(1+|\xi|+|\eta|)^{-(N+n+1+m)} d \xi & \text { if } m<0 .\end{cases}
$$

In any case we find

$$
\left|I_{1}(x, \eta, \tau)\right| \leq C_{N}^{\prime} \frac{|w(\tau /\langle\eta\rangle)|}{(1+|\eta|)^{N}} \leq C_{N}^{\prime} \frac{1}{(\tau+|\eta|)^{N}}, \quad N \in \mathbb{N} .
$$

For the term $I_{2}$ we first write

$$
\begin{aligned}
I_{2}= & w(\tau /\langle\eta\rangle) \phi(x) \omega^{n} e^{-i\langle\kappa(x), \eta\rangle} \\
& \times \iint e^{i \omega(\langle\kappa(x+y), \eta / \omega\rangle-\langle y, \xi\rangle)} a(x, \omega \xi, \tau) \tilde{\phi}(x+y) \chi(\xi) d y d \xi, \quad \omega=|\eta|,
\end{aligned}
$$

to apply the stationary-phase result of Theorem 7.7.7 in [Hör90], which yields, for $k \geq n$,

$$
\begin{aligned}
& \mid I_{2}(x, \eta, \tau)-w(\tau /\langle\eta\rangle) \phi(x) \\
& \quad \times \sum_{\nu=0}^{k-n} \frac{(-i)^{\nu}}{\nu !}\left\langle\partial_{y}, \partial_{\xi} / \omega\right\rangle^{\nu}\left(\left.e^{i \omega\left\langle\rho_{x}(x+y), \eta / \omega\right\rangle} a(x, \omega \xi, \tau) \tilde{\phi}(x+y) \chi(\xi)\right|_{\left.y=0, \xi={ }^{t} \kappa^{\prime}(x) \eta / \omega\right)}\right) \mid \\
& \quad \leq C \omega^{(n-k) / 2} w(\tau /\langle\eta\rangle) \sum_{|\alpha| \leq 2 k} \sup _{y, \xi}\left|D_{y, \xi}^{\alpha}(\tilde{\phi}(x+y) \chi(\xi) a(x, \omega \xi, \tau))\right| .
\end{aligned}
$$

As $\tau \lesssim \omega$, and $\xi$ is bounded, we observe that

$$
\left|\omega^{\alpha}\left(D_{\xi}^{\alpha} a\right)(x, \omega \xi)\right| \lesssim \omega^{|\alpha|}(\tau+\omega|\xi|)^{m-|\alpha|} \lesssim(\tau+\omega)^{m}
$$

We also have $\chi(\xi)=1$ in a neighborhood of ${ }^{t} \kappa^{\prime}(x) \eta / \omega$. As $\phi=1$ and $\tilde{\phi}=1$ in a neighborhood of $K$ we thus obtain

$$
\begin{aligned}
& \mid I_{2}(x, \eta, \tau)-w(\tau /\langle\eta\rangle) \sum_{\nu=0}^{k-n} \frac{(-i)^{\nu}}{\nu !}\left\langle\partial_{y}, \partial_{\xi}\right\rangle^{\nu}\left(\left.e^{i\left\langle\rho_{x}(y), \eta\right\rangle} a(x, \xi, \tau)\right|_{y=x, \xi={ }^{t} \kappa^{\prime}(x) \eta / \omega}\right) \mid \\
& \leq C \omega^{(n-k) / 2}(\tau+\omega)^{m} w(\tau /\langle\eta\rangle) \lesssim(\tau+|\eta|)^{m+n / 2-k / 2}, \quad x \in K .
\end{aligned}
$$

We have thus obtained an asymptotic development in the form of (C.19). As each term in the series is also a semiclassical symbol, by (C.22) we find that an estimate of the form (C.18) is achieved when no derivation is applied to $\gamma_{1}$. Applying partial derivatives with respect to $x$ and $\eta$ to $\gamma_{1}(x, \eta, \tau)$ results in a sum of terms with the same form as (C.20) with additional expressions with at most polynomial growth in $\eta$. The analysis carried out above also yields an estimate of the form (C.18). Together with Lemma C.5 this completes the proof. 
Proof of Proposition C.4. We have

$\gamma_{2}(x, \eta, \tau)=(1-w)(\tau /\langle\eta\rangle) a_{\kappa}(\kappa(x), \xi)$

$=(1-w)(\tau /\langle\eta\rangle) \phi(x) \iint e^{i\langle x-y, \xi\rangle+i\langle\kappa(y)-\kappa(x), \eta\rangle} a(x, \xi, \tau) \tilde{\phi}(y) d y d \xi, \quad x \in X$.

This representation is to be understood in the sense of oscillatory integrals, which justifies the manipulations we perform below.

In the support of $(1-w)(\tau /\langle\eta\rangle)$ we have $\tau \gtrsim|\eta|$. As $\rho_{x}(y)=\kappa(y)-\kappa(x)-$ $\kappa^{\prime}(x)(y-x)$ we write

$\gamma_{2}(x, \eta, \tau)=(1-w)(\tau /\langle\eta\rangle) \phi(x) \iint e^{i\left\langle x-y, \xi-{ }^{t} \kappa^{\prime}(x) \eta\right\rangle+i\left\langle\rho_{x}(y), \eta\right\rangle} a(x, \xi, \tau) \tilde{\phi}(y) d y d \xi$

$=(1-w)(\tau /\langle\eta\rangle) \phi(x) \iint e^{i\langle x-y, \xi\rangle+i\left\langle\rho_{x}(y), \eta\right\rangle} a\left(x, \xi+{ }^{t} \kappa^{\prime}(x) \eta, \tau\right) \tilde{\phi}(y) d y d \xi$,

which by the Taylor formula gives $\gamma_{2}=\gamma_{2, N}+r_{N}$ with

$\gamma_{2, N}(x, \eta, \tau)$

$=\sum_{|\alpha|<N} \frac{1}{\alpha !}(1-w)(\tau /\langle\eta\rangle) \phi(x) \iint e^{i\langle x-y, \xi\rangle+i\left\langle\rho_{x}(y), \eta\right\rangle} \xi^{\alpha} \partial_{\xi}^{\alpha} a\left(x,{ }^{t} \kappa^{\prime}(x) \eta, \tau\right) \tilde{\phi}(y) d y d \xi$

and

$r_{N}=$

$N \sum_{|\alpha|=N} \int_{0}^{1} \frac{(1-\sigma)^{N-1}}{\alpha !} \iint e^{i\langle x-y, \xi\rangle+i\left\langle\rho_{x}(y), \eta\right\rangle} \xi^{\alpha} \partial_{\xi}^{\alpha} a\left(x, \sigma \xi+{ }^{t} \kappa^{\prime}(x) \eta, \tau\right) \tilde{\phi}(y) d y d \xi d \sigma$.

Observing that $\xi^{\alpha} e^{i\langle x-y, \xi\rangle}=i^{|\alpha|} \partial_{y}^{\alpha} e^{i\langle x-y, \xi\rangle}$ we find

$$
\begin{aligned}
\gamma_{2}(x, \eta, \tau)= & (1-w)(\tau /\langle\eta\rangle) \phi(x) \\
& \times \sum_{|\alpha|<N} \frac{(-i)^{|\alpha|}}{\alpha !} \iint e^{i\langle x-y, \xi\rangle} \partial_{y}^{\alpha}\left(\tilde{\phi}(y) e^{i\left\langle\rho_{x}(y), \eta\right\rangle}\right) \partial_{\xi}^{\alpha} a\left(x,{ }^{t} \kappa^{\prime}(x) \eta, \tau\right) d y d \xi \\
= & \left.(1-w)(\tau /\langle\eta\rangle) \phi(x) \sum_{|\alpha|<N} \frac{(-i)^{|\alpha|}}{\alpha !} \partial_{y}^{\alpha}\left(\tilde{\phi}(y) e^{i\left\langle\rho_{x}(y), \eta\right\rangle}\right)\right|_{y=x} \partial_{\xi}^{\alpha} a\left(x,{ }^{t} \kappa^{\prime}(x) \eta, \tau\right) \\
= & \left.(1-w)(\tau /\langle\eta\rangle) \sum_{|\alpha|<N} \frac{(-i)^{|\alpha|}}{\alpha !} \partial_{y}^{\alpha}\left(e^{i\left\langle\rho_{x}(y), \eta\right\rangle}\right)\right|_{y=x} \partial_{\xi}^{\alpha} a\left(x,{ }^{t} \kappa^{\prime}(x) \eta, \tau\right)
\end{aligned}
$$


for $x \in K$, because of the supports of $\phi$ and $\tilde{\phi}$. From the properties of $\rho_{y}(x)$ given in Lemma C.2 each term in the sum is in $S\left(\mu^{m-|\alpha| / 2}\right)$. Similarly we have

$$
\begin{aligned}
& r_{N}(x, \eta, \tau)=(1-w)(\tau /\langle\eta\rangle) \phi(x) N(-i)^{N} \sum_{|\alpha|=N} \sum_{\beta \leq \alpha}\left(\begin{array}{c}
\alpha \\
\beta
\end{array}\right) \int_{0}^{1} \frac{(1-\sigma)^{N-1}}{\alpha !} \\
& \times \iint e^{i\langle x-y, \xi\rangle}\left(\partial_{y}^{\beta-\alpha} \tilde{\phi}(y)\right)\left(\partial_{y}^{\beta} e^{i\left\langle\rho_{x}(y), \eta\right\rangle}\right) \partial_{\xi}^{\alpha} a\left(x, \sigma \xi+{ }^{t} \kappa^{\prime}(x) \eta, \tau\right) d y d \xi d \sigma .
\end{aligned}
$$

If we prove that $r_{N} \lesssim(\tau+|\eta|)^{m+n+1-N / 2}$ if $N \geq m$, we will obtain an estimate of the form (C.19). In particular this yields $\left|\gamma_{2}\right| \lesssim \mu^{v}$ for some $v \in \mathbb{R}$.

Applying partial derivatives with respect to $x$ and $\eta$ to $\gamma_{2}(x, \eta, \tau)$ results in a sum of terms with the same form as (C.23) with additional expressions with at most polynomial growth in $\eta$. Computing $\partial_{x}^{\alpha} \partial_{\eta}^{\beta} \gamma_{2}$ we may apply a similar analysis and find $\left|\partial_{x}^{\alpha} \partial_{\eta}^{\beta} \gamma_{2}\right| \lesssim \mu^{\nu}$ for some $v \in \mathbb{R}$. We thus have an estimate of the form (C.18). In view of Lemma C.5 this will complete the proof.

By Lemma C.2 the remainder term $r_{N}$ in (C.24) is a linear combination of terms of the form

$$
\begin{aligned}
& r_{N}^{\prime}(x, \eta, \tau) \\
& =(1-w)(\tau /\langle\eta\rangle) \phi(x) \int_{0}^{1}(1-\sigma)^{N-1} \iint e^{i\langle x-y, \xi\rangle} \hat{\phi}(y) \prod_{j \in \mathcal{J}}\left\langle\partial_{y}^{\alpha_{j}} \rho_{x}(y), \eta\right\rangle e^{i\left\langle\rho_{x}(y), \eta\right\rangle} \\
& \times \prod_{j \in \mathcal{I}}\left\langle x-y, \rho_{x, j}(y) \eta\right\rangle \partial_{\xi}^{\alpha} a\left(x, \sigma \xi+{ }^{t} \kappa^{\prime}(x) \eta, \tau\right) d y d \xi d \sigma
\end{aligned}
$$

with $\left|\alpha_{j}\right| \geq 2$ if $j \in \mathcal{J}$ and $k=|\mathcal{I}|$ and $\ell=|\mathcal{I}|+|\mathcal{J}|$ such that

$$
k \leq \ell \leq|\beta| \leq|\alpha|=N, \quad \ell \leq(|\beta|+k) / 2 .
$$

Here the function $\hat{\phi}$ has support in $K$ and is constant on $\operatorname{supp}(\phi)$.

As $\left\langle x-y, \rho_{x, j}(y) \eta\right\rangle e^{i\langle x-y, \xi\rangle}=-i\left\langle\partial_{\xi}, \rho_{x, j}(y) \eta\right\rangle e^{i\langle x-y, \xi\rangle}$ we obtain

$$
\begin{aligned}
& r_{N}^{\prime}(x, \eta, \tau) \\
& =i^{k}(1-w)(\tau /\langle\eta\rangle) \phi(x) \int_{0}^{1}(1-\sigma)^{N-1} \iint e^{i\langle x-y, \xi\rangle} \hat{\phi}(y) \prod_{j \in \mathcal{J}}\left\langle\partial_{y}^{\alpha_{j}} \rho_{x}(y), \eta\right\rangle e^{i\left\langle\rho_{x}(y), \eta\right\rangle} \\
& \times \prod_{j \in \mathcal{I}}\left\langle\partial_{\xi}, \rho_{x, j}(y) \eta\right\rangle \partial_{\xi}^{\alpha} a\left(x, \sigma \xi+{ }^{t} \kappa^{\prime}(x) \eta, \tau\right) d y d \xi d \sigma,
\end{aligned}
$$

and we may thus write $r_{N}$ as a linear combination of terms of the form

$$
\begin{aligned}
& r_{N}^{\prime \prime}(x, \eta, \tau) \\
&=(1-w)(\tau /\langle\eta\rangle) \phi(x) \int_{0}^{1} \sigma^{|\gamma|}(1-\sigma)^{N-1} \iint e^{i\langle x-y, \xi\rangle} \hat{\phi}(y) p(x, y, \eta) e^{i\left\langle\rho_{x}(y), \eta\right\rangle} \\
& \times \partial_{\xi}^{\alpha+\gamma} a\left(x, \sigma \xi+{ }^{t} \kappa^{\prime}(x) \eta, \tau\right) d y d \xi d \sigma,
\end{aligned}
$$

where $|\gamma|=k$ and $p(x, y, \eta)$ is a polynomial in $\eta$ of order $\ell$ with smooth coefficients. 
We note that $\langle\xi\rangle^{-2}\left(1+i\left\langle\xi, \partial_{y}\right\rangle\right) e^{i\langle x-y, \xi\rangle}=e^{i\langle x-y, \xi\rangle}$. This yields, for $q \in \mathbb{N}$,

$$
\begin{aligned}
r_{N}^{\prime \prime}(x, \eta, \tau)=(1-w)(\tau /\langle\eta\rangle) \phi & (x) \int_{0}^{1} \sigma^{|\gamma|}(1-\sigma)^{N-1} \\
& \times \iint e^{i\langle x-y, \xi\rangle}\left(1-i\left\langle\xi, \partial_{y}\right\rangle\right)^{q}\left(\hat{\phi}(y) p(x, y, \eta) e^{i\left\langle\rho_{x}(y), \eta\right\rangle}\right) \\
& \times\langle\xi\rangle^{-2 q} \partial_{\xi}^{\alpha+\gamma} a\left(x, \sigma \xi+{ }^{t} \kappa^{\prime}(x) \eta, \tau\right) d y d \xi d \sigma .
\end{aligned}
$$

We choose $|\alpha|=N \geq m$ and $q=n+1$. Then

$$
\left|\partial_{\xi}^{\alpha+\gamma} a\left(x, \sigma \xi+{ }^{t} \kappa^{\prime}(x) \eta, \tau\right)\right| \leq\left(\tau+\left|\sigma \xi+{ }^{t} \kappa^{\prime}(x) \eta\right|\right)^{m-N-|\gamma|} \lesssim \tau^{m-N-|\gamma|} .
$$

We thus obtain

$$
\left|r_{N}^{\prime \prime}(x, \eta, \tau)\right| \leq|(1-w)(\tau /\langle\eta\rangle)||\eta|^{\ell+n+1} \tau^{m-N-|\gamma|} \lesssim \tau^{m+\ell+n+1-N-|\gamma|},
$$

as $m-N-|\gamma| \leq 0$ and $|\eta| \lesssim \tau$. Since $\ell \leq(N+k) / 2=(N+|\gamma|) / 2$ this yields

$$
\left|r_{N}^{\prime \prime}(x, \eta, \tau)\right| \lesssim \tau^{m+n+1-(N+|\gamma|) / 2} \lesssim(\tau+|\eta|)^{m+n+1-N / 2}
$$

as claimed above. This concludes the proof.

\section{C.10. Proof of Proposition B.7}

The proof partly follows the lines of that of [Hör85a, Proposition 18.1.19]. We fix $j \in J$. Let $\tilde{\lambda}_{l}, l \in L$, be a locally finite partition of unity on $\left(0, X_{0}\right) \times \tilde{U}_{j}$. For all $k, l \in L$, we define $\sigma_{k l} \in S_{\mathcal{T}}^{m}\left(\mathbb{R}^{n} \times \mathbb{R} \times \mathbb{R}^{n}\right)$ as

$$
\operatorname{Op}_{\mathcal{T}}\left(\sigma_{k l}\right)=\left(\phi_{j}^{-1}\right)^{*} \lambda_{k} A \phi_{j}^{*} \tilde{\lambda}_{l}, \quad \text { where } \quad \lambda_{k}=\phi_{j}^{*} \tilde{\lambda}_{k}
$$

Note that $\operatorname{supp}\left(\sigma_{k l}\right) \subset\left(0, X_{0}\right) \times \tilde{U}_{j} \times \mathbb{R} \times \mathbb{R}^{n}$. We define $a_{j}:=\sum_{k, l}^{\prime} \sigma_{k l}$ where $\sum^{\prime}$ denotes the sum over the pairs $k, l$ such that $\operatorname{supp}\left(\lambda_{l}\right) \cap \operatorname{supp}\left(\lambda_{k}\right) \neq \emptyset$. This sum is locally finite, which gives $a_{j} \in S_{\mathcal{T}}^{m}\left(\left(0, X_{0}\right) \times \tilde{U}_{j} \times \mathbb{R} \times \mathbb{R}^{n}\right)$.

For $\lambda \in \mathscr{C}_{c}^{\infty}\left(\left(0, X_{0}\right) \times U_{j}\right)$ and $\tilde{\lambda} \in \mathscr{C}_{c}^{\infty}\left(\left(0, X_{0}\right) \times \tilde{U}_{j}\right)$ we consider

$$
\begin{aligned}
R & =\left(\phi_{j}^{-1}\right)^{*} \lambda A \phi_{j}^{*} \tilde{\lambda}-\left(\left(\phi_{j}^{-1}\right)^{*} \lambda\right) \operatorname{Op}_{\mathcal{T}}\left(a_{j}\right) \tilde{\lambda} \\
& =\sum_{k, l}\left(\phi_{j}^{-1}\right)^{*} \lambda \lambda_{k} A \phi_{j}^{*} \tilde{\lambda}_{l} \tilde{\lambda}-\left(\left(\phi_{j}^{-1}\right)^{*} \lambda\right) \mathrm{Op}_{\mathcal{T}}\left(a_{j}\right) \tilde{\lambda}
\end{aligned}
$$

Note that the sum only involves $k, l$ such that $\operatorname{supp}\left(\lambda_{k}\right) \cap \operatorname{supp}(\lambda) \neq \emptyset$ and $\operatorname{supp}\left(\tilde{\lambda}_{l}\right) \cap$ $\operatorname{supp}(\tilde{\lambda}) \neq \emptyset$. Hence, the sum is finite. We find

$$
R=\sum_{k, l}^{\prime}\left(\phi_{j}^{-1}\right)^{*} \lambda \lambda_{k} A \phi_{j}^{*} \tilde{\lambda}_{l} \tilde{\lambda}-\left(\left(\phi_{j}^{-1}\right)^{*} \lambda\right) \mathrm{Op}_{\mathcal{T}}\left(a_{j}\right) \tilde{\lambda}+R_{1}
$$


where $R_{1}$ is a finite sum of operators in $\Psi_{\mathcal{T}}^{m}\left(\mathbb{R}^{n} \times \mathbb{R}\right)$ (and also in $\Psi_{\mathcal{T}}^{m}\left(\left(0, X_{0}\right) \times \tilde{U}_{j} \times \mathbb{R}\right)$ ) with kernels vanishing in a neighborhood of the diagonal. By Lemma B.2, we have $R_{1} \in$ $h^{\infty} \Psi_{\mathcal{T}}^{-\infty}\left(\mathbb{R}^{n} \times \mathbb{R}\right)$. Moreover,

$$
\left(\phi_{j}^{-1}\right)^{*} \lambda \lambda_{k} A \phi_{j}^{*} \tilde{\lambda}_{l} \tilde{\lambda}=\left(\left(\phi_{j}^{-1}\right)^{*} \lambda\right) \mathrm{Op}_{\mathcal{T}}\left(\sigma_{k l}\right) \tilde{\lambda} .
$$

Thus $R=R_{1}$ from the definition of $a_{j}$.

We now prove uniqueness. Let $\tilde{a}_{j}$ have the same properties as $a_{j}$. Introducing $b=$ $a_{j}-\tilde{a}_{j}$, for all $\lambda \in \mathscr{C}_{c}^{\infty}\left(\left(0, X_{0}\right) \times U_{j}\right)$ and $\tilde{\lambda} \in \mathscr{C}_{c}^{\infty}\left(\left(0, X_{0}\right) \times \tilde{U}_{j}\right)$ we have

$$
\left(\left(\phi_{j}^{-1}\right)^{*} \lambda\right) \operatorname{Op}_{\mathcal{T}}(b) \tilde{\lambda} \in h^{\infty} \Psi_{\mathcal{T}}^{-\infty}\left(\mathbb{R}^{n} \times \mathbb{R}\right) .
$$

Let $K$ be a compact set in $\left(0, X_{0}\right) \times \tilde{U}_{j}$ and choose $\lambda, \tilde{\lambda}$ such that $\left(\phi_{j}^{-1}\right)^{*} \lambda=1$ on $K$ and $\tilde{\lambda}=1$ on $\operatorname{supp}\left(\left(\phi_{j}^{-1}\right)^{*} \lambda\right)$. The symbol of $\left(\left(\phi_{j}^{-1}\right)^{*} \lambda\right) \operatorname{Op}_{\mathcal{T}}(b) \tilde{\lambda}$ is in $h^{\infty} S_{\mathcal{T}}^{-\infty}\left(\mathbb{R}^{n} \times \mathbb{R} \times \mathbb{R}^{n}\right)$ and is given by

$$
\left(\left(\phi_{j}^{-1}\right)^{*} \lambda\right) b \# \tilde{\lambda} \in\left(\phi_{j}^{-1}\right)^{*} \lambda b+h^{\infty} S_{\mathcal{T}}^{-\infty}\left(\mathbb{R}^{n} \times \mathbb{R} \times \mathbb{R}^{n}\right)
$$

by the composition formula (B.1). As a consequence, according to Definition B.3,

$$
b \in h^{\infty} S_{\mathcal{T}}^{-\infty}\left(\left(0, X_{0}\right) \times \tilde{U}_{j} \times \mathbb{R} \times \mathbb{R}^{n}\right) .
$$

\section{C.11. Proof of Proposition B.8}

Let $K$ be a compact set in $\left(0, X_{0}\right) \times\left(U_{j} \cap U_{k}\right)$. Let $\lambda, \hat{\lambda} \in \mathscr{C}_{c}^{\infty}\left(\left(0, X_{0}\right) \times\left(U_{j} \cap U_{k}\right)\right)$ be equal to 1 on $K$. We set $\tilde{\lambda}_{\ell}=\left(\phi_{\ell}^{-1}\right)^{*} \hat{\lambda}, \ell=j, k$. We also introduce $A_{\lambda, \hat{\lambda}, \ell}=$ $\left(\phi_{\ell}^{-1}\right)^{*} \lambda A \phi_{\ell}^{*} \tilde{\lambda}_{\ell}$ and find

$$
A_{\lambda, \hat{\lambda}, \ell}=\operatorname{Op}_{\mathcal{T}}\left(\tilde{a}_{\ell}\right) \bmod h^{\infty} \Psi_{\mathcal{T}}^{-\infty}\left(\mathbb{R}^{n} \times \mathbb{R}\right) \quad \text { with } \quad \tilde{a}_{\ell}=\left(\left(\phi_{\ell}^{-1}\right)^{*} \lambda\right) a_{\ell} \# \tilde{\lambda}_{\ell}, \ell=j, k .
$$

The kernel of $A_{\lambda, \hat{\lambda}, j}$ (resp. $A_{\lambda, \hat{\lambda}, k}$ ) has compact support in $\left(\left(0, X_{0}\right) \times \tilde{U}_{j, k}\right)^{2}$ (resp. $\left(\left(0, X_{0}\right) \times \tilde{U}_{k, j}\right)^{2}$.) Observe that

$$
A_{\lambda, \hat{\lambda}, k}=\left(\phi_{j k}^{-1}\right)^{*} A_{\lambda, \hat{\lambda}, j} \phi_{j k}^{*} .
$$

From Theorem B.1, we have, for all $N \in \mathbb{N}$,

$$
\tilde{a}_{k}-T_{\phi_{j k}, N}\left(\tilde{a}_{j}\right) \in h^{N} S_{\mathcal{T}}^{m-N / 2}\left(\mathbb{R}^{n} \times \mathbb{R} \times \mathbb{R}^{n}\right) .
$$

Set $K_{k}=\phi_{k}(K)$ and pick $\chi \in \mathscr{C}_{c}^{\infty}\left(K_{k}\right)$. Since $\lambda=\hat{\lambda}=1$ on $K$, we have

$$
\chi a_{k}=\chi \tilde{a}_{k} \bmod h^{\infty} S_{\mathcal{T}}^{-\infty}\left(\mathbb{R}^{n} \times \mathbb{R} \times \mathbb{R}^{n}\right) .
$$

We also have

$$
\begin{aligned}
\chi \tilde{a}_{k} & =\chi T_{\phi_{j k}, N}\left(\tilde{a}_{j}\right) \bmod h^{N} S_{\mathcal{T}}^{m-N / 2}\left(\mathbb{R}^{n} \times \mathbb{R} \times \mathbb{R}^{n}\right) \\
& =\chi T_{\phi_{j k}, N}\left(a_{j}\right) \bmod h^{N} S_{\mathcal{T}}^{m-N / 2}\left(\mathbb{R}^{n} \times \mathbb{R} \times \mathbb{R}^{n}\right),
\end{aligned}
$$


because of the form of $T_{\phi_{j k}, N}$ in (B.5). We thus obtain

$$
\chi\left(a_{k}-T_{\phi_{j k}, N}\left(a_{j}\right)\right) \in h^{N} S_{\mathcal{T}}^{m-N / 2}\left(\mathbb{R}^{n} \times \mathbb{R} \times \mathbb{R}^{n}\right) .
$$

As $K$ is arbitrary, (C.26) holds for any $\chi \in \mathscr{C}_{c}^{\infty}\left(\tilde{U}_{k, j}\right)$. This gives the conclusion according to Definition B.3.

\section{C.12. Proof of Proposition B.10}

Let $K_{A, h}$ and $K_{B, h}$ be the kernels of $A$ and $B$. We shall use the notation of Definition B.5. As the two operators are properly supported, the composition makes sense and $A B$ : $\mathscr{C}_{c}^{\infty}(\mathcal{X}) \rightarrow \mathscr{C}_{c}^{\infty}(\mathcal{X})$. We denote its distribution kernel by $K_{A B, h}$. (Note that we use the Riemannian structure here to identify functions, densities, and half-densities on $\mathcal{X}$.) We have

$$
\mathcal{K}_{A B, h}\left(x_{0}, y ; \hat{x}_{0}, \hat{y}\right)=\iint_{\mathcal{X}^{\prime}} \mathcal{K}_{A, h}\left(x_{0}, y ; \check{x}_{0}, \check{y}\right) \mathcal{K}_{B, h}\left(\check{x}_{0}, \check{y} ; \hat{x}_{0}, \hat{y}\right) d \check{x}_{0} d \check{y}
$$

in the sense given at the end of Section 8.2 in [Hör90]. We choose $\chi, \hat{\chi} \in \mathscr{C}_{c}^{\infty}\left(\mathcal{X}^{\prime}\right)$ such that $\operatorname{supp}(\chi) \cap \operatorname{supp}(\hat{\chi})=\emptyset$. In addition we introduce $\check{\chi}$ such that $\operatorname{supp}(\chi) \cap \operatorname{supp}(\check{\chi})=\emptyset$ and $\check{\chi}=1$ on $\operatorname{supp}(\hat{\chi})$. We then write

$$
\begin{aligned}
& \chi\left(x_{0}, y\right) \hat{\chi}\left(\hat{x}_{0}, \hat{y}\right) \mathcal{K}_{A B, h}\left(x_{0}, y ; \hat{x}_{0}, \hat{y}\right) \\
& =\hat{\chi}\left(\hat{x}_{0}, \hat{y}\right) \iint_{\mathcal{X}^{\prime}} \chi\left(x_{0}, y\right) \check{\chi}\left(\check{x}_{0}, \check{y}\right) \mathcal{K}_{A, h}\left(x_{0}, y ; \check{x}_{0}, \check{y}\right) \mathcal{K}_{B, h}\left(\check{x}_{0}, \check{y} ; \hat{x}_{0}, \hat{y}\right) d \check{x}_{0} d \check{y} \\
& \quad+\chi\left(x_{0}, y\right) \iint_{\mathcal{X}^{\prime}} \hat{\chi}\left(\hat{x}_{0}, \hat{y}\right)\left(1-\check{\chi}\left(\check{x}_{0}, \check{y}\right)\right) \mathcal{K}_{A, h}\left(x_{0}, y ; \check{x}_{0}, \check{y}\right) \mathcal{K}_{B, h}\left(\check{x}_{0}, \check{y} ; \hat{x}_{0}, \hat{y}\right) d \check{x}_{0} d \check{y} .
\end{aligned}
$$

In the first term, $\chi\left(x_{0}, y\right) \check{\chi}\left(\check{x}_{0}, \breve{y}\right) \mathcal{K}_{A, h}\left(x_{0}, y ; \check{x}_{0}, \check{y}\right)$ is smooth and compactly supported because of the disjoint supports of the cut-off functions and the regularity of the kernel $\mathcal{K}_{A, h}$ off the diagonal. In the second term, $\hat{\chi}\left(\hat{x}_{0}, \hat{y}\right)\left(1-\check{\chi}\left(\check{x}_{0}, \check{y}\right)\right) \mathcal{K}_{B, h}\left(\check{x}_{0}, \check{y} ; \hat{x}_{0}, \hat{y}\right)$ is also smooth as $\operatorname{supp}(\hat{\chi}) \cap \operatorname{supp}(1-\check{\chi})=\emptyset$, and compactly supported as $\mathcal{K}_{B, h}$ is properly supported. Because of (B.8) both terms then yield a smooth function in the variables $x_{0}, y, \hat{x}_{0}, \hat{y}$ and estimating derivatives then yields a proper estimate of the form of (B.7).

We now consider $j \in J$ and $\lambda \in \mathscr{C}_{c}^{\infty}\left(\left(0, X_{0}\right) \times U_{j}\right), \tilde{\lambda} \in \mathscr{C}_{c}^{\infty}\left(\left(0, X_{0}\right) \times \tilde{U}_{j}\right)$. We set

$$
\alpha=\left(\phi_{j}^{-1}\right)^{*} \lambda A B \phi_{j}^{*}(\tilde{\lambda}) .
$$

We then introduce $\chi, \hat{\chi} \in \mathscr{C}_{c}^{\infty}\left(\left(0, X_{0}\right) \times U_{j}\right)$ such that $\chi=\hat{\chi}=1$ on $\operatorname{supp}\left(\phi_{j}^{*} \tilde{\lambda}\right)$. We write $\alpha=\beta+R$ with

$$
\beta=\left(\phi_{j}^{-1}\right)^{*} \lambda A \chi \hat{\chi} B \phi_{j}^{*}(\tilde{\lambda}), \quad R=\left(\phi_{j}^{-1}\right)^{*} \lambda A(1-\chi \hat{\chi}) B \phi_{j}^{*}(\tilde{\lambda}) .
$$

Arguing as above we find that the kernel $K_{R}$ of $R$ is a smooth function and it satisfies an estimate of the form $\sup _{x_{n}} q\left(K_{R}\right) \leq C h^{N}$ for any $N \in \mathbb{N}$ and any seminorm $q$ on $\mathscr{C}^{\infty}\left(\left(\mathbb{R}^{n} \times \mathbb{R}\right)^{2}\right)$. Moreover its support is compact. Hence $R \in h^{\infty} \Psi_{\mathcal{T}}^{-\infty}\left(\mathbb{R}^{n} \times \mathbb{R}\right)$.

Next, with $\tilde{\chi}=\left(\phi_{j}^{-1}\right)^{*} \chi$ we write

$$
\beta=\left(\phi_{j}^{-1}\right)^{*} \lambda A \phi_{j}^{*} \tilde{\chi}\left(\phi_{j}^{-1}\right)^{*} \hat{\chi} B \phi_{j}^{*}(\tilde{\lambda}) .
$$


By Proposition B.7 we obtain a semiclassical tangential operator on $\mathbb{R}^{n} \times \mathbb{R}$ with symbol

$$
\beta_{j}=\left(\left(\left(\phi_{j}^{-1}\right)^{*} \lambda\right) a_{j}\right) \#\left(\tilde{\chi}\left(\left(\phi_{j}^{-1}\right)^{*} \hat{\chi}\right) b_{j}\right) \# \tilde{\lambda} \bmod h^{\infty} S_{\mathcal{T}}^{-\infty}\left(\mathbb{R}^{n} \times \mathbb{R}\right),
$$

which belongs to $S_{\mathcal{T}}^{m+m^{\prime}}\left(\mathbb{R}^{n} \times \mathbb{R}\right)$. The operator $A B$ is thus in $\Psi_{\mathcal{T}}^{m+m^{\prime}}\left(\mathbb{R}^{n} \times \mathbb{R}\right)$.

From the composition formula B.1, because of the supports of $\tilde{\chi}$ and $\left(\phi_{j}^{-1}\right)^{*} \hat{\chi}$ we further obtain

$$
\beta_{j}=\left(\left(\phi_{j}^{-1}\right)^{*} \lambda\left(a_{j} \# b_{j}\right)\right) \# \tilde{\lambda} \bmod h^{\infty} S_{\mathcal{T}}^{-\infty}\left(\mathbb{R}^{n} \times \mathbb{R}\right) .
$$

Hence by Proposition B.7, $a_{j} \# b_{j}$ is a representative of the local symbol of $A B$ in this chart.

\section{C.13. Proof of Proposition B.12}

The existence of $L$ is only related to the proper support of the kernel of $A$. We have

$$
\left|(A u)_{\mid x_{n}=0}\right|_{k}=\sum_{j}\left|\left(\phi_{j}^{-1}\right)^{*} \psi_{j}(A u)_{\mid x_{n}=0}\right|_{k} .
$$

Let $j \in J$. It suffices to prove that

$$
\left|\left(\phi_{j}^{-1}\right)^{*} \psi_{j}(A u)_{\mid x_{n}=0}\right|_{k} \leq C_{K}|u|_{k+\ell} .
$$

We choose a partition of unity $\sum_{k} \hat{\psi}_{k}=1$, subordinated to the open covering $\left(U_{k}\right)_{k \in J}$ such that $\hat{\psi}_{j}=1$ in a neighborhood of $\operatorname{supp}\left(\psi_{j}\right)$. Then $\operatorname{supp}\left(\hat{\psi}_{k}\right) \cap \operatorname{supp}\left(\psi_{j}\right)=\varnothing$ for $k \neq j$. We then have

$$
\psi_{j} A u=\sum_{k \neq j} \psi_{j} A \hat{\psi}_{k} u+\psi_{j} A \hat{\psi}_{j} u
$$

The terms in the sum are then associated with properly supported operators with smooth kernels for which the operator continuity (after restriction to $x_{n}=0$ ) is clear. To treat the last term we choose $\lambda \in \mathscr{C}_{c}^{\infty}\left(\left(0, X_{0}\right) \times U_{j}\right)$ such that $\lambda=1$ on $L \cap\left(\left(0, X_{0}\right) \times \operatorname{supp}\left(\psi_{j}\right)\right)$, and $\hat{\lambda} \in \mathscr{C}_{c}^{\infty}\left(\left(0, X_{0}\right) \times \tilde{U}_{j}\right)$ such that $\hat{\lambda}=1$ on $\left.K \cap\left(\left(0, X_{0}\right) \times \operatorname{supp}\left(\left(\phi_{j}^{-1}\right)^{*} \psi_{j}\right)\right)\right)$. Then

$$
\left(\phi_{j}^{-1}\right)^{*} \psi_{j} A \hat{\psi}_{j} u=\left(\phi_{j}^{-1}\right)^{*} \lambda \psi_{j} A \phi_{j}^{*} \hat{\lambda}\left(\phi_{j}^{-1}\right)^{*} \hat{\psi}_{j} u=B\left(\phi_{j}^{-1}\right)^{*} \hat{\psi}_{j} u \quad \text { at } x_{n}=0,
$$

with $B \in \Psi_{\mathcal{T}}^{\ell}\left(\mathbb{R}^{n+1}\right)$ by Definition B.5. Hence

$$
\left|\left(\phi_{j}^{-1}\right)^{*} \psi_{j}\left(A \hat{\psi}_{j} u\right)_{\mid x_{n}=0}\right|_{k} \lesssim\left|\left(\phi_{j}^{-1}\right)^{*} \hat{\psi}_{j} u\right|_{k+\ell} \lesssim|u|_{k+\ell}
$$

by $(1.22)$.

Acknowledgments. The authors wish to thank E. Zuazua for suggesting the study of the controllability of a model with a diffusive interface. Most of this research was carried out at Institut Henri Poincaré (Paris, France). Moreover, the authors wish to thank Institut Henri Poincaré for providing a very stimulating environment during the "Control of Partial Differential Equations and Applications" program in the Fall 2010.

The authors were partially supported by l'Agence Nationale de la Recherche under grant ANR07-JCJC-0139-01. 


\section{References}

[Bel03] Bellassoued, M.: Carleman estimates and distribution of resonances for the transparent obstacle and application to the stabilization. Asymptotic Anal. 35, 257-279 (2003) Zbl 1137.35388 MR 2011790

[BDL07a] Benabdallah, A., Dermenjian, Y., Le Rousseau, J.: Carleman estimates for the onedimensional heat equation with a discontinuous coefficient and applications to controllability and an inverse problem. J. Math. Anal. Appl. 336, 865-887 (2007) Zbl 1189.35349 MR 2352986

[BDL07b] Benabdallah, A., Dermenjian, Y., Le Rousseau, J.: On the controllability of linear parabolic equations with an arbitrary control location for stratified media. C. R. Math. Acad. Sci. Paris 344, 357-362 (2007) Zbl 1115.35055 MR 2310670

[BDL11] Benabdallah, A., Dermenjian, Y., Le Rousseau, J.: Carleman estimates for stratified media. J. Funct. Anal. 260, 3645-3677 (2011) Zbl 1218.35238 MR 2781972

[BGL07] Benabdallah, A., Gaitan, P., Le Rousseau, J.: Stability of discontinuous diffusion coefficients and initial conditions in an inverse problem for the heat equation. SIAM J. Control Optim. 46, 1849-1881 (2007) Zbl 1155.35485 MR 2361996

[Bre83] Brezis, H.: Analyse Fonctionnelle. Masson, Paris (1983) Zbl 0511.46001 MR 0697382

[Bur98] Burq, N.: Décroissance de l'énergie locale de l'équation des ondes pour le problème extérieur et absence de résonance au voisinage du réel. Acta Math. 180, 1-29 (1998) Zbl 0918.35081 MR 1618254

[DOP02] Doubova, A., Osses, A., Puel, J.-P.: Exact controllability to trajectories for semilinear heat equations with discontinuous diffusion coefficients. ESAIM Control Optim. Calc. Var. 8, 621-661 (2002) Zbl 1092.93006 MR 1932966

[GS94] Grigis, A., Sjöstrand, J.: Microlocal Analysis for Differential Operators. Cambridge Univ. Press, Cambridge (1994) Zbl 0804.35001 MR 1269107

[Hör63] Hörmander, L.: Linear Partial Differential Operators. Springer, Berlin (1963) Zbl 0108.09301 MR 0161012

[Hör79] Hörmander, L.: The Weyl calculus of pseudo-differential operators. Comm. Pure Appl. Math. 32, 359-443 (1979) Zbl 0388.47032 MR 0517939

[Hör85a] Hörmander, L.: The Analysis of Linear Partial Differential Operators. Vol. III, Springer (1985) Zbl 0601.35001 MR 0781536

[Hör85b] Hörmander, L.: The Analysis of Linear Partial Differential Operators. Vol. IV, Springer (1985) Zbl 0612.35001 MR 0781537

[Hör90] Hörmander, L.: The Analysis of Linear Partial Differential Operators. Vol. I, 2nd ed., Springer (1990) Zbl 0712.35001 MR 1065136

[JL99] Jerison, D., Lebeau, G.: Nodal sets of sums of eigenfunctions. In: Harmonic Analysis and Partial Differential Equations (Chicago, IL, 1996), Chicago Lectures in Math., Univ. of Chicago Press, Chicago, 223-239 (1999) Zbl 0946.35055 MR 1743865

[KZ06] Koch, H., Zuazua, E.: A hybrid system of PDE's arising in multi-structure interaction: coupling of wave equations in $n$ and $n-1$ space dimensions. In: Recent Trends in Partial Differential Equations, Contemp. Math. 409, Amer. Math. Soc., 55-77 (2006) Zbl 1108.35110 MR 2243949

[Léa10] Léautaud, M.: Spectral inequalities for non-selfadjoint elliptic operators and application to the null-controllability of parabolic systems. J. Funct. Anal. 258, 2739-2778 (2010) Zbl 1185.35153 MR 2593342

[Leb96] Lebeau, G.: Équation des ondes amorties. In: Algebraic and Geometric Methods in Mathematical Physics (Kaciveli, 1993), Math. Phys. Stud. 19, Kluwer, Dordrecht, 73109 (1996) Zbl 0863.58068 MR 1385677 
[LR95] Lebeau, G., Robbiano, L.: Contrôle exact de l'équation de la chaleur. Comm. Partial Differential Equations 20, 335-356 (1995) Zbl 0819.35071 MR 1312710

[LR97] Lebeau, G., Robbiano, L.: Stabilisation de l'équation des ondes par le bord. Duke Math. J. 86, 465-491 (1997) Zbl 0884.58093 MR 1432305

[LZ98] Lebeau, G., Zuazua, E.: Null-controllability of a system of linear thermoelasticity. Arch. Ration. Mech. Anal. 141, 297-329 (1998) Zbl 1064.93501 MR 1620510

[Ler10] Lerner, N.: Metrics on the Phase Space and Non-Selfadjoint Pseudo-Differential Operators. Birkhäuser, Basel (2010) Zbl 1186.47001 MR 2599384

[Le 07] Le Rousseau, J.: Carleman estimates and controllability results for the one-dimensional heat equation with $B V$ coefficients. J. Differential Equations 233, 417-447 (2007) Zbl 1128.35020 MR 2292514

[LL12] Le Rousseau, J., Lebeau, G.: On Carleman estimates for elliptic and parabolic operators. Applications to unique continuation and control of parabolic equations. ESAIM Control Optim. Calc. Var. 18, 712-747 (2012) Zbl pre06111871

[LL11] Le Rousseau, J., Lerner, N.: Carleman estimates for anisotropic elliptic operators with jumps at an interface. Anal. PDE, to appear

[LR10] Le Rousseau, J., Robbiano, L.: Carleman estimate for elliptic operators with coefficients with jumps at an interface in arbitrary dimension and application to the null controllability of linear parabolic equations. Arch. Ration. Mech. Anal. 105, 953-990 (2010) Zbl 1202.35336 MR 2591978

[LR11] Le Rousseau, J., Robbiano, L.: Local and global Carleman estimates for parabolic operators with coefficients with jumps at interfaces. Invent. Math. 183, 245-336 (2011) Zbl 1218.35054 MR 2772083

[LZ12] Lescarret, V., Zuazua, E.: Numerical approximation schemes for multi-dimensional wave equations in asymmetric spaces. Preprint (2012)

[Mar02] Martinez, A.: An Introduction to Semiclassical and Microlocal Analysis. Springer (2002) Zbl 0994.35003 MR 1872698

[Mil06] Miller, L.: On the controllability of anomalous diffusions generated by the fractional laplacian. Math. Control Signals Systems 3, 260-271 (2006) Zbl 1105.93015 MR 2272076

[Mil10] Miller, L.: A direct Lebeau-Robbiano strategy for the observability of heatlike semigroups. Discrete Contin. Dynam. Systems Ser. B 14, 1465-1485 (2010) Zbl 1219.93017 MR 2679651

[Paz83] Pazy, A.: Semigroups of Linear Operators and Applications to Partial Differential Equations. Springer, New York (1983) Zbl 0516.47023 MR 0710486

[Rob95] Robbiano, L.: Fonction de coût et contrôle des solutions des équations hyperboliques. Asymptotic Anal. 10, 95-115 (1995) Zbl 0882.35015 MR 1324385

[Tay81] Taylor, M. E.: Pseudodifferential Operators. Princeton Univ. Press, Princeton, NJ (1981) Zbl 0453.47026 MR 0618463

[TT11] Tenenbaum, G., Tucsnak, M.: On the null-controllability of diffusion equations. ESAIM Control Optim. Calc. Var. 17, 1088-1100 (2011) Zbl 1236.93025 MR 2859866 